Sciences pour

la commun!cation

\title{
La variation pluridimensionnelle
}

\section{Une analyse de la négation en français}

Charlotte Meisner 
Cet ouvrage présente une nouvelle approche originelle à la vielle question de la variation du ne de négation en français moderne. Soigneusement établie sur un corpus de langue parlée, l'auteur présente l'hypothèse de la variation linguistique pluridimensionnelle : le clitique négatif ne est parfois réalisé, comme dans la phrase ma mère ne vient pas, mais très souvent omis, surtout dans la communication informelle : je viens pas. Comme toute variable linguistique, le ne de négation est soumis à un ensemble d'influences potentielles. À l'aide d'une analyse multifactorielle, Charlotte Meisner montre que la variation pluridimensionnelle du ne de négation est déterminée par un facteur-clé sous-jacent : la prosodie du français moderne.

Charlotte Meisner est collaboratrice scientifique à l'université de Zurich. Après avoir étudié les philologies romanes et les sciences politiques aux universités d'Hanovre, de Bologne et de Berlin, elle a fait sa thèse de doctorat en linguistique française à l'université de Zurich. 


\section{La variation pluridimensionnelle}




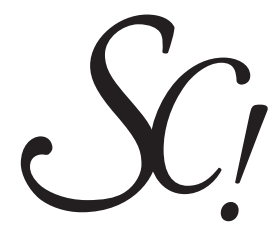

\title{
Sciences pour la communication
}

\author{
Vol. 118
}

\section{Comité scientifique}

D. Apothéloz, Université de Nancy 2

J.-P. Bronckart, Université de Genève

P. Chilton, Université de Lancaster

W. De Mulder, Université d'Anvers

J.-P. Desclés, Université Paris-Sorbonne

F.H. van Eemeren, Université d'Amsterdam

V. Escandell-Vidal, UNED, Madrid

F. Gadet, Université de Paris Ouest Nanterre La Défense

J.-M. Marandin, CNRS et Université Paris-Diderot

F. Martineau, Université d'Ottawa

M. Milton Campos, Université de Montréal

J. Rouault, Université Stendhal (Grenoble 3)

Les ouvrages publiés dans cette collection ont été sélectionnés par les soins du comité éditorial, après révision par les pairs.

Collection publiée sous la direction de

Marie-José Béguelin, Alain Berrendonner,

Didier Maillat et Louis de Saussure 


\section{Charlotte Meisner}

\section{La variation pluridimensionnelle}

Une analyse de la négation en français

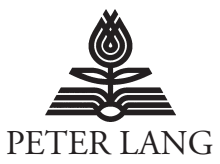

Bern • Berlin • Bruxelles $•$ Frankfurt am Main • New York • Oxford • Wien 


\section{Information bibliographique publiée par « Die Deutsche Nationalbibliothek »}

«Die Deutsche Nationalbibliothek » répertorie cette publication dans la « Deutsche

Nationalbibliografie "; les données bibliographiques détaillées sont disponibles sur Internet sous ‘http://dnb.d-nb.de».

Die vorliegende Arbeit wurde von der Philosophischen Fakultät der Universität Zürich im Herbstsemester 2013 auf Antrag der Promotionskommission:

Prof. Dr. Elisabeth Stark (hauptverantwortliche Betreuungsperson), Prof. em. Dr. Georg Bossong als Dissertation angenommen.

Cette publication a été soutenue par le Fonds National Suisse de la recherche scientifique.

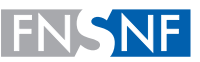

FONDS NATIONAL SUISSE DE LA RECHERCHE SCIENTIFIQUE

Graphisme : Atelier 4b, Sandra Meyer

ISBN 978-3-0343-2085-6 br. • ISBN 978-3-0352-0347-9 eBook

ISSN 0933-6079 br. • ISBN 978-3-0343-2333-8 MOBI

ISBN 978-3-0343-2332-1 ePUB • DOI 10.3726/978-3-0352-0347-9

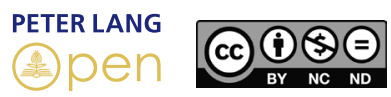

Open Access: Cette œuuvre est mise à disposition selon les termes de la Licence Creative Commons Attribution - Pas d'Utilisation Commerciale - Pas de Modification 4.0.

Pour consulter une copie de cette licence, visitez le site internet https://creativecommons.org/licenses/by-nc-nd/4.0/

Cette publication a fait l'objet d'une évaluation par les pairs.

(c) Peter Lang SA, Editions scientifiques internationales, Berne 2016

Wabernstrasse 40, CH-3007 Berne, Suisse

bern@peterlang.com, www.peterlang.com 
Pour Grossi

Je tiens à remercier ma famille et mes amis pour le soutien qu'ils m'ont apporté. 
Charlotte Meisner - 978-3-0343-2332-1

Downloaded from PubFactory at 01/11/2019 02:38:59AM

via free access 
»Raus mit der Sprache, Doktor - was ist das?«

»Das ist, äh, eine Doktorarbeit.«

»Eine Doktorarbeit? «lachte Smeik.»Jetzt bin ich aber erleichtert.

Ich dachte schon, es sei eine schreckliche Krankheit.."

»Das ist eine Doktorarbeit gewissermaßen auch. [...] Doktorarbeiten können in vielen Gestalten erscheinen [...] eine Doktorarbeit besteht zum großen Teil aus anderen Doktorarbeiten«, erläuterte Kolibril. »Eine neue Doktorarbeit ist immer auch eine Art Orgie von alten Doktorarbeiten, die sich untereinander, äh, befruchten, damit etwas Neues, etwas noch nie Dagewesenes aus ihnen hervorgeht."

Walter Moers »Rumo \& die Wunder im Dunkeln« (2009, Piper, München, S. 142-145) 
Charlotte Meisner - 978-3-0343-2332-1

Downloaded from PubFactory at 01/11/2019 02:38:59AM

via free access 


\section{Table des matières}

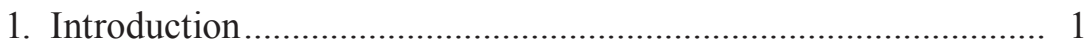

1.1 La variation \pm ne de la négation en français ............................ 1

1.2 La conception, les objectifs et la structure du livre ................ 8

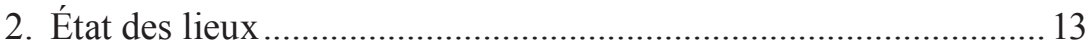

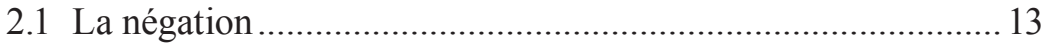

2.1.1 Généralités ................................................................ 13

2.1.2 L'évolution de la négation en français.............................. 19

2.1.2.1 Le cycle de Jespersen ......................................... 19

2.1.2.2 Changement rapide ou variation stable? ............23

2.1.3 La négation en français moderne......................................27

2.1.3.1 Les particules négatives ne et pas .....................22

2.1.3.2 Les termes négatifs et de polarité.........................30

2.2 La variable \pm ne dans les analyses empiriques ...........................4 42

2.2.1 \pm ne dans les corpus graphiques........................................ 42

2.2.1.1 \pm ne dans les textes du $17^{\mathrm{e}}$ au $19^{\mathrm{e}}$ siècle ............. 42

2.2.1.2 \pm ne dans la communication électronique au $20^{\mathrm{e}}$ et $21^{\mathrm{e}}$ siècle .............................................44

2.2.2 $\pm n e$ en français contemporain: les analyses

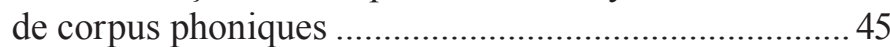

2.2.3 Tendances générales: l'influence des facteurs (extra)linguistiques sur $\pm n e$..............................50

2.2.4 L'influence des facteurs extralinguistiques

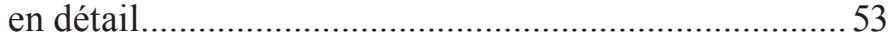

2.2.4.1 La diatopie ..................................................... 53

2.2.4.2 L'âge des locuteurs ............................................5

2.2.4.3 La diastratie: la formation et la provenance sociale des locuteurs....................57

2.2.4.4 La variation situationnelle...................................59 
2.2.5 L'influence des facteurs linguistiques en détail 62

2.2.5.1 Le type de sujet grammatical: un continuum quant à la variable $\pm n e$.

2.2.5.2 Discussion du type de sujet grammatical:

un continuum morphosyntaxique

et phonologique 68

2.2.5.3 Les proclitiques non-sujets ............................ 74

2.2.5.4 L'élément négatif non-clitique .......................... 77

2.2.5.5 Les temps et modes verbaux ............................ 78

2.2.5.6 Le type de phrase: l'opposition entre principale et subordonnée .......................79

2.2.5.7 Les influences phonologiques ......................... 81

2.2.6 Discussion critique des analyses empiriques de \pm ne.... 83

2.2.7 Conclusion intermédiaire: quelques implications pour notre analyse de corpus ...................................... 85

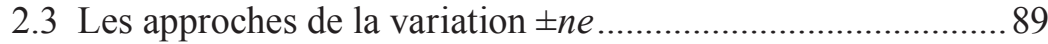

2.3.1 L'approche externe: \pm ne comme variable sociolinguistique

2.3.2 L'approche interne: l'hypothèse de l'incompatibilité entre $n e$ et d'autres clitiques....................................... 91

2.3.3 L'approche diglossique: l'hypothèse des deux grammaires du français

2.3.4 L'approche pragmatique: ne comme une particule emphatique............................................. 103

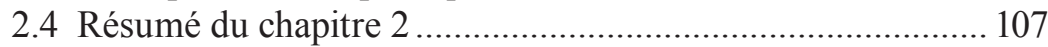

3. Méthodologie: le corpus ....................................................... 113

3.1 Comment accéder au corpus ? ......................................... 113

3.2 Le corpus: motivation, conception et possibilités de requête.... 113

3.3 Description sociodémographique de T-zéro...........................116

3.4 Description des situations d'enregistrement ...................... 121

3.4.1 La distance communicative: l'examen oral................ 122

3.4.2 L'immédiat communicatif: conversations et discussions ............................................................ 125

3.5 Le protocole d'enregistrement........................................... 128 
3.6 Le protocole de transcription .............................................. 130

3.7 Le protocole pour l'analyse de la variable \pm ne dans T-zéro .... 132

3.8 Les tests statistiques pour l'analyse descriptive................... 135

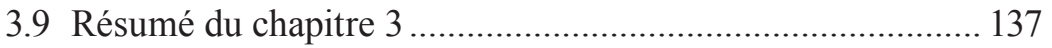

4. L'analyse de corpus : \pm ne dans le corpus T-zéro .......................... 139

4.1 Analyse globale: \pm ne dans le corpus T-zéro......................... 139

4.2 Analyse exploratrice: \pm ne selon le type de locuteur............. 140

4.2.1 Type A: les locuteurs qui montrent une

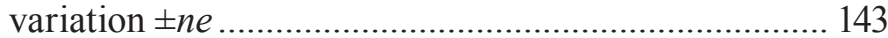

4.2.2 Type B: négation monopartite uniquement ................. 144

4.2.3 Type $C$ : négation bipartite uniquement ...................... 148

4.3 Analyse descriptive de l'influence des facteurs extralinguistiques ......................................... 150

4.3.1 La diatopie ....................................................... 150

4.3.2 L'âge des locuteurs................................................. 152

4.3.3 La diastratie: la profession et la formation

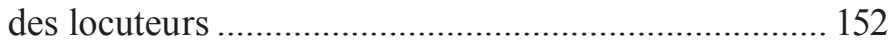

4.3.4 Locuteurs bilingues et monolingues.......................... 153

4.3.5 La variation situationnelle ....................................... 155

4.3.6 Conclusion intermédiaire: l'influence des facteurs extralinguistiques .................................. 163

4.4 Analyse descriptive de l'influence

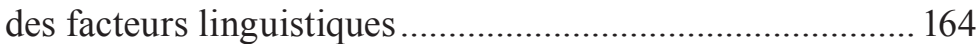

4.4.1 L'influence du type de sujet ...................................... 164

4.4.1.1 Les sujets lourds .......................................... 166

4.4.1.2 Les sujets légers......................................... 170

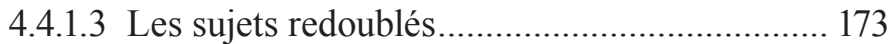

4.4.2 Les séquences proclitiques ...................................... 177

4.4.3 L'élément négatif non-clitique.................................... 180

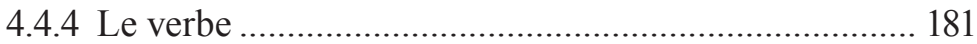

4.4.5 Le type de phrase.................................................. 187

4.4.5.1 L'opposition entre les principales et les subordonnées....................................... 187

4.4.5.2 Les constructions interrogatives et clivées ..... 189 
4.4.6 Le contexte phonétique

4.4.7 Conclusion intermédiaire: l'influence des facteurs linguistiques

4.5 Analyse multifactorielle

4.5.1 L'interaction entre $\pm n e$ et une série de variables dites 'indépendantes'

4.5.2 L'interaction entre les variables dites 'indépendantes' dans le corpus entier

4.5.2.1 L'influence de la situation de communication sur l'emploi du type de sujet...........................206

4.5.2.2 L'influence de la situation de communication sur le type de phrase..................................... 210

4.5.3 Conclusion intermédiaire.......................................... 211

4.6 Résumé du chapitre 4 ...................................................... 212

5. Discussion des résultats ........................................................ 217

5.1 Évaluation des approches de la variation $\pm n e$

face aux résultats de l'analyse ........................................... 217

5.1.1 L'approche externe: \pm ne comme variable sociolinguistique

5.1.2 L'approche interne: l'hypothèse de l'incompatibilité entre ne et d'autres clitiques.

5.1.3 L'approche diglossique: l'hypothèse des deux grammaires du français

5.1.4 L'approche pragmatique: ne comme une particule emphatique....

5.1.5 L'approche du contact linguistique.... 234

5.1.6 Conclusion intermédiaire. 236

5.2 Proposition d'une explication prosodique de $\pm n e$ en français 238

5.2.1 Pourquoi une explication prosodique? 238

5.2.2 La prosodie du français 239

5.2.3 Le modèle prosodique de Jun/Fougeron (2000) 242

5.2.4 Application à \pm ne. 245

5.2.4.1 L'influence prosodique des sujets légers 
5.2.4.2 L’influence prosodique des séquences proclitiques ...................................................248

5.2.4.3 L'influence prosodique des sujets lourds.........254

5.2.4.4 L'influence prosodique des sujets redoublés ... 257

5.2.4.5 L'influence prosodique du type de phrase....... 259

5.2.4.6 L'applicabilité de l'approche prosodique à d'autres contextes négatifs ........................... 261

5.2.4.7 Conclusion intermédiaire ............................... 271

5.3 La variation situationnelle $\mathrm{du} \pm n e$ comme épiphénomène des facteurs prosodiques ................................................... 274

5.3.1 La nature de la variation situationnelle \pm ne ............... 274

5.3.2 Pourquoi la variation situationnelle $\pm n e$ est-elle un épiphénomène? ......................................... 276

5.3.3 D’autres facteurs pertinents liés à la situation de communication.................................................... 281

5.3.4 Les implications théoriques pour le traitement

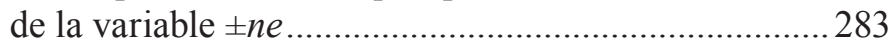

5.4 Perspectives ....................................................................... 285

5.4.1 L'explication prosodique et d'autres approches

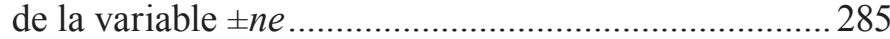

5.4.3 Quel futur pour le ne de négation?........................... 289

5.5 Résumé du chapitre 5 ....................................................... 292

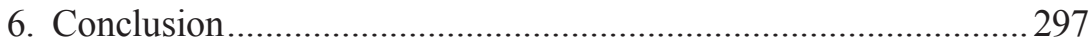

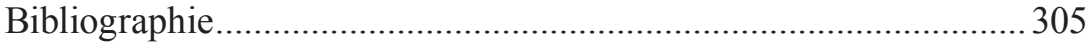

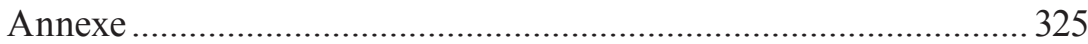


Charlotte Meisner - 978-3-0343-2332-1

Downloaded from PubFactory at 01/11/2019 02:38:59AM

via free access 


\section{Introduction}

\subsection{La variation $\pm n e$ de la négation en français}

Toutes les langues humaines permettent la transformation d'une phrase affirmative en une phrase négative. La négation, une opération sémantique et syntaxique qui exprime le renversement de la valeur de vérité d'une proposition, est donc une propriété cognitive universelle du langage humain qui n'existe dans aucun système de communication animal. La négation est profondément enracinée dans la communication humaine et nous permet de contredire, de répliquer, de rejeter, de mentir, d'ironiser ou de corriger (cf. Horn 2010: 1, Horn/ Kato 2000: 1). En ce qui concerne l'expression de la négation dans les langues humaines, il existe une variation considérable non seulement entre différentes langues, mais également entre les différentes étapes diachroniques d'une langue, voire simultanément à l'intérieur d'une même langue.

Le présent travail se propose d'analyser la variation linguistique observée dans l'expression de la négation du verbe fini en français phonique ${ }^{1}$ moderne:

1 Nous nous servons de la terminologie développée par Koch/Oesterreicher (2011: 5-6), basée sur Söll (21980: 11). Koch/Oesterreicher (22011: 5-6) font une distinction entre le médium (phonique/graphique) et la conception (de l'immédiat) de la distance communicative) d'un énoncé. Étant donné que nous analyserons uniquement le code phonique issu aussi bien des situations de l'immédiat que de la distance communicative, l'expression français phonique nous semble plus exacte que d'autres notions courantes comme le 'français parlé', 'français oral', 'français colloquial' ou 'français familier', qui évoquent une conception plutôt informelle. 
(1) La variation \pm ne en français moderne

a. je ne sais pas $(1463)^{2}$

b. je sais pas (0037)

La négation de phrase en français standard (cf. Gaatone 1971, Muller 1991, Rowlett 1998) est exprimée par la combinaison des particules ne et pas ou par ne et un autre élément négatif, comme personne, rien, plus, jamais etc., qui encadrent le verbe fléchi, comme dans l'exemple (1)a.

Cependant, de nombreuses analyses de corpus confirment que la majorité des négations de phrase en français phonique est exprimée sans ne et uniquement par pas ou par des éléments négatifs nonclitiques, comme l'exemple (1)b le montre (cf. entre autres Armstrong 2002, Armstrong/Smith 2002, Ashby 1976, 1981, 2001, Coveney 22002, Culbertson 2010, Diller 1983, Dufter/Stark 2007, Fonseca-Greber 2007, Hansen/Malderez 2004, Lüdicke 1982, Meisner 2010, Pohl 1968, Pooley 1996, Sturm 1981, van Compernolle 2009).

$\mathrm{Au}$ niveau diachronique, la variation $\pm n e$ illustrée sous (1) est décrite en termes d'une disparition continue de la particule ne au cours des derniers siècles. La particule proclitique est peu à peu remplacée par des éléments négatifs toniques et souvent postverbaux, qui, à leur tour, sont susceptibles de subir le même sort que ne, ce qui provoque une évolution dite 'cyclique', comme le montre la citation de Jespersen $(1917,1924)$ :

(2) L'adverbe négatif [ne, C.M.] est souvent inaccentué parce qu'un autre mot de la phrase porte l'accent principal. Mais, lorsque la négation inaccentuée en arrive à n'être plus qu'une syllabe proclitique et même à se réduire à un son unique, on ressent le besoin de la renforcer par l'adjonction d'un autre mot; ce mot est à son tour perçu au bout d'un certain moment comme la négation

2 Sauf si autrement spécifié, les exemples cités dans ce travail proviennent de notre corpus $T$-zéro (nommé d'après la position du verbe fléchi en syntaxe générative, cf. chapitre 3), qui est accessible en ligne sous <http://server.linguistik.uzh. ch/cmeisner2013-tzero> (login: «TZ-Reader», mot de passe «TZero@ling!»). Les exemples peuvent être repérés à l'aide du numéro d'identification à quatre chiffres: cliquez sur Suchen, copiez-collez le numéro dans la cellule $I D C V$ et cliquez sur Perform Find pour afficher l'entrée complète de l'exemple en question, ou consultez le manuel d'utilisation <en ligne> au même endroit. 
elle-même et peut alors subir la même évolution que l'élément qui l'avait précédé. On obtient ainsi une perpétuelle oscillation entre l'affaiblissement et le renforcement de la négation (Jespersen 1992 [1924]: 479).

Dans une perspective synchronique, le cycle négatif observé par Jespersen (1992 [1924]: 479) donne lieu en français contemporain à la variation $\pm n e$ illustrée en (1), qui est décrite et analysée au cours du $20^{\mathrm{e}}$ siècle par des grammairiens, par la linguistique variationnelle et variationniste ${ }^{3}$ ainsi que par des syntacticiens.

Damourette/Pichon (1911-1927: 129-146), par exemple, voient avant tout dans l'omission de $n e$ une caractéristique du 'parler vulgaire'. Ils thématisent ses éventuelles origines phonétiques et ses conséquences sémantiques et en viennent à conclure que la possibilité de l'omission de ne prouve que ce sont désormais les éléments négatifs non-clitiques qui expriment la négation de phrase. Le Bidois/Le Bidois (1938) avancent, quant à eux, des raisons structurelles pour expliquer l'omission de $n e$ : outre sa réduction phonétique, l'analogie avec les expressions sans ne (cf. pas toujours, découragée jamais, Le Bidois/Le Bidois 1938: 657-658) aurait déclenché cette évolution. Les ouvrages didacticopragmatiques et descriptifs modernes ne manquent pas non plus d'attirer l'attention du lecteur sur l'omission de ne dans le langage 'informel', 'parlé', 'familier' ou 'oral' (Lang/Perez 1996: 223) et de mentionner son lien avec l'évolution de la négation (cf. Grevisse/Goosse ${ }^{15} 2011$ : $\S 1022-1015$, Wilmet ${ }^{2} 1998,2007$, Riegel/Pellat/Rioul ${ }^{5} 2008$ : 415-418).

En faisant référence aux premiers travaux se focalisant sur le 'français populaire' de Bauche (1951), Frei (1929) et Müller/Elsass (1985), la linguistique variationnelle de tradition européenne, basée sur des auteurs comme Coseriu (1988a,b), Flydal (1951) et Söll (21980), et représentée récemment par les travaux de Koch/Oesterreicher ( $\left.{ }^{2} 2011\right)$, conçoit l'absence de ne comme une caractéristique centrale du 'français parlé’ contemporain. Comme le français y est conçu en tant que

3 Nous empruntons à Gadet $(2009,172)$ la distinction terminologique entre linguistique variationnelle, pour désigner une tradition théorique instaurée par Coseriu (1988a,b), et linguistique variationniste quant à l'approche quantitative au sens établi par Labov (1966). 
langue historique, c'est-à-dire un ensemble complexe, formé de variétés linguistiques qui se situent sur les dimensions diatopique, diastratique, diaphasique et éventuellement sur le continuum entre l'immédiat et la distance communicative, l'omission de ne serait, dans l'optique de Koch/Oesterreicher (2011), un trait propre à la variété historiquement évoluée du 'français parlé' qui se situe dans le domaine de l'immédiat communicatif.

Les analyses variationnnistes et sociolinguistiques visent à décrire d'un point de vue quantitatif et empirique le cheminement du changement prévu par Jespersen $(1917,1924)$ à travers la société et la langue. Ces études de corpus arrivent souvent à la conclusion que la prédiction de Jespersen $(1917,1924)$ se confirme actuellement en français contemporain. Ashby (1981 : 686), par exemple, affirme à cet égard: «The variable incedence of ne across the several groups of speakers, together with the historical record, suggests that the particle is now being lost in spoken French». Néanmoins, il existe aussi des voix contraires à la perte définitive de $n e$ : Hansen/Malderez (2004: 26) signalent qu'un tel aboutissement serait encore très éloigné, car «les locuteurs se trouvent exposés à l'usage de ce ne dans une diversité de contextes qui le retiennent certainement dans leur système linguistique».

Indépendamment du sort que l'on conçoit pour $n e$, quelques informations concernant la distribution de la variable linguistique $\pm n e$ à l'intérieur de la société et la langue sont à retenir. Tout d'abord, la négation bipartite semble plus stablement enracinée au sud qu'au nord de la France ( $c f$. Diller 1983), tandis qu'elle est relativement rare en Suisse ( $c f$. Fonseca-Greber 2007) et déjà presque inexistante en français canadien (cf. Poplack/St-Amand 2009, Sankoff/Vincent 1980). Sociologiquement parlant, ce sont surtout les jeunes qui font avancer le changement (cf. Armstrong 2002, Pooley 1996), c'est-à-dire que, même s'ils remanient leur production de ne au au cours de leur vie en direction de la norme, les adolescents d'aujourd'hui n'atteignent plus les taux de $+n e$ de leurs parents. Jusqu'aux années 1980, des différences de \pm ne entre différentes classes sociales et niveaux de formation pouvaient être observées ( $c f$. Ashby 1976, 1981, Sturm 1981), mais depuis quelque temps, celles-ci semblent s'estomper ( $c f$. Coveney ${ }^{2} 2002$, Hansen/Malderez 
2004). Contrairement aux facteurs démographiques, le facteur de la situation de communication, c'est-à-dire le facteur du style ou du registre, récemment (re)découvert par la recherche sociolinguistique ( $c f$. Eckert/ Rickford 2001, Coupland 2007), semble toujours valable en français contemporain, du moins pour les adultes.

Au-delà des facteurs sociodémographiques cités-ci dessus, le contexte linguistique s'est avéré extrêmement significatif pour la réalisation de la variable $\pm n e$ : les analyses de corpus ( $c f$. Armstrong/Smith 2002, Ashby 1976, 1981, 2001, Coveney 22002, Hansen/Malderez 2004 etc.) ont montré de manière consistante que le type de sujet est le facteur le plus important pour la réalisation de ne. En simplifiant quelque peu, nous pouvons dire que le patron de variation \pm ne par rapport à ce facteur habituellement observé est le suivant:

(3) \pm ne selon le type de sujet: le patron de variation généralement observé dans les corpus

\begin{tabular}{|c|c|c|}
\hline Sujet & $\pm n e$ & Exemple du corpus \\
\hline Lexical & $+n e$ & a. Ulysse ne montre aucun intérêt dans le dialogue (0046) \\
\hline Pronom clitique & $-n e$ & b. $\boldsymbol{j}$ 'ai pas grand chose à dire (0134) \\
\hline Redoublé & $-n e$ & c. moi je vais pas lire en allemand (0664) \\
\hline
\end{tabular}

Les trois exemples sous (3) illustrent une tendance existant dans tous les corpus de français spontané: les locuteurs tendent à réaliser la particule de négation ne lorsque celle-ci suit un sujet grammatical prosodiquement lourd, comme c'est le cas du nom propre Ulysse en (3)a. Les syntagmes lexicaux, les pronoms indéfinis, comme personne ou quelqu'un, et, à un moindre degré, les pronoms relatifs comme qui ont un effet similaire: plus ils sont prosodiquement lourds, plus ils favorisent la réalisation de ne. Par contre, si le sujet est un clitique prosodiquement léger, comme le pronom personnel je en (3)b, ou si le sujet est redoublé, c'est-à-dire formé par deux entités coréférentielles dont l'une est prosodiquement lourde et l'autre légère, comme moi je dans l'exemple (3)c, la particule ne sera très probablement omise.

En plus de l'influence du type de sujet, d'autres facteurs linguistiques ont été identifiés comme ayant une influence considérable sur 
la variable \pm ne (cf. Armstrong/Smith 2002, Ashby 1976, 1981, 2001, Coveney 22002, Hansen/Malderez 2004 etc.). Il s'agit, par exemple, du temps verbal, de l'élément négatif non-clitique, de la construction syntaxique, du débit de parole et du contexte phonologique. Les analyses de corpus ont montré que ce sont avant tout les variantes fréquentes, comme le présent de l'indicatif, l'élément négatif pas, les phrases simples, le débit rapide et l'omission du schwa, qui favorisent l'omission de $n e$.

La théorie variationnelle et syntaxique moderne offre au moins quatre conceptions de la variation en question. Selon l'approche sociolinguistique 'classique', \pm ne dépend de facteurs externes à la langue. Les sociolinguistes soutiennent notamment qu'il s'agit d'une variable sociolinguistique (cf. Coveney ${ }^{2} 2002$ : 29-54, Gadet 1997a,b, 2007, Labov 1927a,b, 2001), ce qui signifie qu'elle est censée manifester une covariation stable avec des facteurs sociodémographiques comme l'âge ou la couche sociale des locuteurs. Par contre, selon l'approche interne (cf. Larrivée 2014, Posner 1985: 189), tne dépendrait de régularités propres à la langue, comme par exemple d'une 'contrainte clitique' qui exclurait sa réalisation en présence d'autres proclitiques. L'approche diglossique (cf. Culbertson 2010, Massot 2010, Mensching 2008 et ZribiHertz 2011), en partie compatible avec les approches de la linguistique variationnelle, comme celle de Koch/Oesterreicher (2011), rapporte la variation $\pm n e$ au fait que la particule serait présente dans une variété $d u$ français de distance mais pas dans le français de l'immédiat et que les francophones alterneraient continuellement entre ces deux grammaires. L'absence de ne serait donc, tout comme d'autres variantes linguistiques, une caractéristique de cette variété de l'immédiat, acquise de manière inconsciente et naturelle, de génération en génération, comme première grammaire, tandis que la variété de distance serait apprise tardivement et imparfaitement à l'école et à travers les contextes de protocole (par exemple dans le contact avec les institutions officielles, à l'église etc.). En combinant les idées d'une contrainte clitique et d'une diglossie francophone, Culbertson (2010) affirme même que les proclitiques du français de l'immédiat seraient désormais des marques flexionnelles affixées qui excluraient la présence du clitique ne. Finale- 
ment, les adhérents de l'approche pragmatique, basée sur les travaux de Bell $(1984,2001)$ et soutenue récemment par van Compernolle (2008a,b), Fonseca-Greber (2007) et Poplack/St-Amand (2009), surtout par rapport au français canadien et suisse, approuvent l'hypothèse selon laquelle ne est une particule d'emphase, employée uniquement dans certains contextes pragmatiquement définis. Les observations de Breitbarth/Haegeman (2010, à paraître) concernant l'ancienne particule négative en du flamand de l'ouest suivent cette approche.

La plupart de ces approches pourraient être intégrées dans les modèles syntaxiques génératifs à travers des règles qui opèrent la négation bi- ou monopartite, par exemple dans la grammaire de certains groupes de locuteurs, dans les contextes proclitiques ou pragmatiquement définis ainsi que dans les variétés de la distance et de l'immédiat communicatif ( $c f$. les propositions de Dubois 1967: 137 et Jones ${ }^{3} 2007$ : 348 dans un cadre génératif, et de Knüppel 2001 dans la grammaire d'unification).

Cependant, de telles solutions théoriques 'minimales' admettent l'existence de la variation $\pm n e$ sans l'expliqueren profondeur. En effet, l'intégration des informations sur la variation \pm e dont nous disposons grâce aux analyses de corpus reste généralement faible dans les approches théoriques. L'un des objectifs de ce travail sera donc d'essayer d'intégrer les différentes approches et d'apporter ainsi de nouveaux éléments de réponse quant à la variation $\pm n e$.

C'est notamment à cause de la multitude des approches théoriques et méthodologiques de la variable $\pm n e$, abordées brièvement ci-dessus, et des nombreux facteurs linguistiques et extralinguistiques qui semblent l'influencer, que nous la concevons comme une variable pluridimensionnelle. Le besoin de comprendre la complexité de la variation $\pm n e$ sera donc au cœur de la conception de notre analyse. 


\subsection{La conception, les objectifs et la structure du livre}

Cette thèse de doctorat, composée de six chapitres, envisage quatre objectifs principaux: la description, la documentation, l'analyse et l'explication de la variation $\pm n e$ dans la négation de phrase en français phonique contemporain.

Après cette brève introduction au sujet de la variation $\pm n e$, nous commencerons par proposer, dans le chapitre 2, une description syntaxique de la négation en français contemporain en tenant compte des données historiques et typologiques. Ceci comprend également la présentation structurée des hypothèses existantes et des résultats obtenus quant à la variable \pm ne en diachronie et en synchronie.

Le second objectif concerne la documentation de la variable $\pm n e$ dans la négation de phrase en français contemporain. Notre corpus T-zéro de français phonique spontané (d'environ 16150 mots), décrit dans le chapitre 3, cible la variation diasituationnelle et diatopique et se compose de données linguistiques issues de deux situations d'enregistrement classées à l'aide du modèle de la distance et de l'immédiat communicatif de Koch/Oesterreicher (2011). La première situation de communication comprend des données d'examens oraux, tandis que la deuxième contient des transcriptions de conversations et de discussions en groupes. Les deux situations étant enregistrées pour moitié en Île-de-France et pour moitié à Neuchâtel, en Suisse romande, la structure du corpus est quadripartite: il contient des données de l'immédiat communicatif de Suisse (25\%) et de France (25\%) ainsi que de la distance communicative des deux pays (respectivement $25 \%$ des données totales).

Le corpus T-zéro comprend uniquement des constructions verbales (positives et négatives), c'est-à-dire que nous n'avons retenu dans chaque entrée de la base de données qu'un verbe fléchi avec ses arguments (le sujet et les compléments). Chaque énoncé est transcrit deux fois, à savoir orthographiquement et phonétiquement, comme le montre l'exemple (4). Ceci permet une analyse plus approfondie de l'influence du contexte phonétique sur la variable $\pm n e$ que celle effectuée habituellement dans 
les analyses de corpus à ce sujet (cf. Armstrong/Smith 2002, Ashby 1976, 1981, 2001, Coveney 22002, Hansen/Malderez 2004 etc.).

(4) Le système de double transcription

a. j'aime pas parler des langues (1905)

b. [зєmpapasledelãg]

En nous basant sur ce corpus, nous nous proposons, en tant que troisième objectif, de combiner l'analyse descriptive 'classique' de nombreux facteurs (extra)linguistiques influençant la variable $\pm n e$, telle qu'elle est habituellement utilisée dans ce type de travaux ( $c f$. Armstrong/Smith 2002, Ashby 1976, 1981, 2001, Coveney ${ }^{2}$ 2002, Hansen/Malderez 2004 etc.), avec des analyses exploratrices et multifactorielles qui permettent de former des groupes de locuteurs en fonction de leur comportement langagier, de mesurer le poids respectif des divers facteurs en question et de tirer de cette manière des conclusions concernant leurs interactions.

Les interdépendances entre les facteurs intra- et extralinguistiques pertinentes pour \pm ne étant au cœur de la conception de cette étude, nous analyserons, dans le chapitre 4 , la réalisation de la variable dans toutes les négations de phrases comprises dans notre corpus en fonction des nombreux facteurs (extra)linguistiques montrés dans le tableau (5). Nous chercherons à établir tout d'abord le comportement linguistique de chaque locuteur quant à $\pm n e$, puis l'influence respective des facteurs intra- et extralinguistiques, et les différentes interactions entre ceux-ci.

(5) Les variantes (extra)linguistiques retenues dans T-zéro et illustrées à travers l'exemple 1905 : j'aime pas parler des langues [зєmpapabledelãg]

\begin{tabular}{|l|lll|}
\hline \multicolumn{3}{|l}{ Variable } & Variante \\
\hline Linguistique & Verbe & Forme du verbe fléchi & $\mathrm{\varepsilon m}$ \\
& & Infinitif du verbe & aimer \\
& & Temps du verbe & indicatif présent \\
& Arguments & Sujet & je réalisé [3] \\
& & Complément infinitival & VP parler des langues \\
\cline { 2 - 4 } & Négation & Réalisation de \pm ne & -ne \\
& & Terme négatif & pas \\
\cline { 2 - 4 } & Construction syntaxique & Principale \\
\hline
\end{tabular}




\begin{tabular}{|l|ll|}
\hline Extralinguistique & Sexe & Masculin \\
& Âge & $19-24$ \\
& Langue(s) maternelle(s) & Français \\
\cline { 2 - 3 } & Suisse (Neuchâtel) \\
& Provenance & Étudiant à l'université \\
& Niveau d'études & Informelle (conversa- \\
& Situation d'enregistrement & tion dans la cuisine) \\
\hline
\end{tabular}

Enfin, après une discussion approfondie des résultats de l'analyse et de la pertinence des hypothèses existantes dans ce contexte, notre quatrième objectif concernera l'explication prosodique de la variation de $\pm n e$, qui sera approfondie dans le chapitre 5 et qui représente en quelque sorte un retour aux premières analyses de la variation $\pm n e$. En effet, tout comme Jespersen $(1917,1924)$, nous plaçons au centre de notre explication la nature clitique de ne, c'est-à-dire son caractère phonologiquement et syntaxiquement faible. Au lieu de proclamer l'existence de régularités sociolinguistiques, syntaxiques ou pragmatiques complexes, nous argumenterons tout simplement que la réalisation de $\pm n e$ dépend du contexte prosodique qui le précède, ce qui permet l'intégration de plusieurs autres facteurs morphosyntaxiques et discursifs dans cette même approche, qui sera implémentée à l'aide du modèle prosodique de Jun/Fougeron (2000).

Plus précisément, nous soutenons que, d'une part, les sujets prosodiquement lourds, comme les syntagmes lexicaux, les pronoms indéfinis, et les clitiques 'lourds' tels que nous, vous et elle, favorisent la variante $+n e$, car ils peuvent porter l'accent initial, que le français semble posséder en plus de l'accent final principal ( $c f$. Hirst/Di Cristo 1996, Jun/Fougeron 2000 : 210, Vaissière 1997, Welby 2003 : 11). D'autre part, les sujets clitiques 'légers', comme $j e, t u, i l$, ce et on, favorisent la variante -ne parce que comme ne ils sont généralement inaccentuables et parce que les séquences de plusieurs éléments inaccentués contredisent le principe eurythmique ( $c f$. Dell 1984, Di Cristo 1998: 197), qui gouverne la prosodie du français.

L'opposition entre les constructions principales et subordonnées a un effet similaire mais moins fort que celui du sujet: tandis que les sub- 
ordonnées, dont l'élément initial est une conjonction de subordination, peuvent souvent porter l'accent initial sur cet élément, les principales comportant un sujet clitique n'offrent aucune position pour cet accent, ce qui provoque une réduction des séquences proclitiques, qui, à leur tour, ont une influence directe sur la réalisation de $n e$.

Quant aux contextes négatifs non-finis ou impératifs, le taux élevé de $+n e$ en présence de participes présents et de gérondifs ainsi que dans les infinitives est explicable à travers le même mécanisme prosodique: un seul élément faible (dans ces cas ne) est admis dans l'attaque d'un groupe accentuel, tandis que des séquences proclitiques y sont tendanciellement évitées.

À l'aide de l'approche prosodique, la variation stylistique ou situationnelle de $\pm n e$ peut être expliquée comme un épiphénomène de la distribution des types de sujets grammaticaux et de phrase: d'une part, dans la distance communicative, la présence élevée des sujets lourds et des constructions subordonnées provoque une réalisation assez stable de ne; d'autre part, dans les situations de l'immédiat communicatif, où les sujets clitiques et les principales sont particulièrement fréquents, ceux-ci provoquent l'absence presque totale de la particule de négation.

La relation entre le facteur structural du type de sujet et la nature du discours que nous évoquons ici est statistiquement vérifiable et a été observée dans de nombreuses langues ( $c f$. la notion de Preferred Argument Structure, Du Bois 1987, 2003, Lambrecht 1987). Son importance pour l'explication de la variation $\pm n e$ a été reconnue pour la première fois par Dufter/Stark (2007: 120), qui notent: «Étant donné que seulement les sujets lexicaux encouragent l'emploi du ne, son omission particulièrement fréquente à l'oral ne nous surprend guère» ( $c f$. aussi la discussion d'Armstrong/Smith 2002: 34-35). Cependant, cette observation n'a pas encore été intégrée de façon systématique dans une approche théorique, comme nous le proposerons ici.

$\mathrm{Vu}$ que l'absence et la présence de ne peuvent être identifiées comme un épiphénomène de la distribution des facteurs linguistiques qui les déterminent, le traitement de cette variable en tant que caractéristique de certains groupes de locuteurs, comme le soutiennent les 
sociolinguistes (cf. Coveney ${ }^{2} 2002$ : 29-54, Gadet 1997a,b, 2007, Labov 1972a,b, 2001), ou en tant que variété du français de l'immédiat, comme le proposent Koch/Oesterreicher (2011), semble moins évident. Finalement, les scénarios qui prévoient une disparition complète et rapide de ne en français, comme celui d'Ashby (1981: 686) par exemple, sont eux aussi démentis par les faits empiriques et théoriques présentés dans ce travail: compte tenu de la présence élevée que $n e$ atteint avec les sujets lourds, nous sommes amenés à conclure que cette particule est toujours bien enracinée dans la grammaire du français. Sa rareté dans les corpus serait donc alors uniquement due à la fréquence généralement faible des variantes linguistiques qui la favorisent.

Les hypothèses et les résultats centraux de cette thèse seront résumés dans le chapitre 6 , où nous présenterons également des pistes de recherche futures. 


\section{2. État des lieux}

\subsection{La négation}

\subsubsection{Généralités}

Dans toutes les langues humaines, les présuppositions discursives, c'est-à-dire les suppositions du locuteur sur ce que son interlocuteur croit, attend ou favorise (cf. Givón 1978: 70), des énoncés négatifs sont plus marquées que celles de leurs contreparties affirmatives. De plus, les constructions négatives se trouvent dans des environnements discursifs pragmatiquement plus complexes, car elles contredisent une présupposition ou une assertion explicite 4 .

D'un point de vue logique, la négation lie deux éléments qui sont réciproquement incompatibles ( $c f$. Ladusaw 1996: 321, Krifka 1995). Ainsi le principe de contradiction prédit qu'une proposition $p$ et sa négation $\neg p$ (ou $\sim p$ ) ne peuvent être vraies au même moment ( $c f$. «Law of contadiction», Horn ${ }^{2} 2001$ : 18-21).

Bien que l'opposition contradictoire puisse être exprimée par un opérateur linguistique de négation (en français, les particules négatives $\pm n e \ldots p a s)$, il est important de noter que la négation dans les langues naturelles n'équivaut pas à la négation logique. Tandis qu'en logique

4 Givón (1978: 79-80) explique ce fait à l'aide de l'exemple suivant: il est très improbable qu'une conversation entre deux amis soit ouverte par l'énoncé négatif ma femme n'est pas enceinte, tandis que l'affirmation ma femme est enceinte semble une nouvelle tout à fait digne d'être mentionnée, même au début d'une conversation. Pour commencer une conversation avec un énoncé négatif, le locuteur doit supposer que son interlocuteur dispose de certaines informations (par exemple d'une grossesse planifiée) ou que l'interlocuteur a implicitement ou explicitement exprimé une supposition (par exemple que la femme était enceinte). 
deux négations consécutives donnent automatiquement un sens affirmatif ( $\neg \neg p=p$ ), comme non nullus ('certains') en latin classique, ceci n'est pas nécessairement le cas dans toutes les langues naturelles (cf. Bernini/Ramat 1996: 2). De plus, la négation dans les langues naturelles permet de combiner la quantification existentielle $(\exists)$ et universelle $(\forall)$ : quelqu'un rit est équivalent à pas tous ne rient pas (noté: $\exists \mathrm{x}[\operatorname{rire}(\mathrm{x})] \equiv \neg \forall \mathrm{x}[\neg \operatorname{rire}(\mathrm{x})])\left(c f\right.$. Bußmann ${ }^{4} 2008$ : 468).

En français, comme dans toutes les langues humaines, il existe diverses possibilités pour exprimer la négation ( $c f$. Ducrot 1972, Moeschler 1982). L'objectif du présent travail étant d'analyser l'occurrence variable de la particule ne dans la négation de phrase en français moderne, nous proposons tout d'abord une classification qui permet de distinguer la négation de phrase d'autres types de négation.

En français contemporain, la négation de phrase (NDP), illustrée en (6), est exprimée syntaxiquement par la combinaison de la particule $n e$, si elle est réalisée, et pas ou un autre élément négatif, par exemple un indéfini négatif ${ }^{5}$, comme personne ou rien, ou un adverbe négatif, comme jamais ou plus (cf. section 2.1.3.2), et porte sur le noyau du prédicat:
(6) La négation de phrase (NDP)
a. elle est pas là (0001)
b. vous avez vu personne ici en haut ce matin (0563)
c. parce qu'il a rien d'autre après (0208)
d. il ne répond jamais à Cornélius (0070)
e. je sais plus si c'est diamésique ou diamérique (0319)

5 En accord avec Jäger (2008: 21) et de Swart (2010: 11), et contrairement à Muller (1991), Riegel/Pellat/Rioul (52008) et Larrivée (2004), nous supposons que la négation par un indéfini négatif est à interpréter comme négation de phrase, car la portée de la négation concerne toute la phrase. Muller (1991) considère les indéfinis négatifs comme «semi-négations» ( $c f$. Muller 1991: 249), alors que Riegel/Pellat/Rioul (52008) les appellent «négation partielle» (cf. Riegel/Pellat/ Rioul 52008: 411-412). 
Au niveau syntaxique, la négation de phrase s'oppose à la négation de constituant (NDC) $)^{6}$, qui figure sous (7):

(7) La négation de constituant (NDC)

a. C'est un comédien pas drôle.

Peters (1999: 116)

b. je trouve dommage de ne pas [/] de ne pas s'exprimer (C-ORAL-ROM)

Le critère de distinction ${ }^{7}$ entre les deux types de négation est sa portée: la négation de phrase porte sur le noyau du prédicat, donc sur le verbe fléchi, comme en (6), et la négation de constituant porte sur un autre élément ( $c f$. Penka 2011: 8), comme par exemple sur l'adjectif drôle en (6)a (où l'emploi de ne est impossible) ou sur l'infinitif s'exprimer en (6)b.

$\mathrm{Au}$ niveau sémantique, la négation de phrase peut être interprétée comme négation interne de l'assertion seule ou comme négation externe de l'assertion et de la présupposition:

(8) La négation interne vs. externe

(Arrivé et al. 1986: 397)

a. Les enfants $\left(n^{\prime}\right)$ ont pas cassé les vitres.

(Négation interne de l'assertion, présuppositions maintenues)

b. Il est faux que les enfants aient cassé les vitres.

(Négation externe de l'assertion et de la présupposition)

En (8)a, un cas de négation interne pertinent pour notre analyse, les présuppositions que les enfants et les vitres existent et que les vitres ont été cassées sont maintenues. La négation externe, introduite en (8)b par

6 Nous utilisons ici les termes de négation de phrase et de constituant au lieu de la paire terminologique ambigüe négation totale et partielle, qui est parfois utilisée dans une acception similaire, mais qui s'applique également à la distinction entre les particules de négation (ne...pas), les adverbes (jamais, plus) (cf. Wagner/ Pichon 1991 : 417-418) et les pronoms négatifs (personne, rien) (cf. Riegel/Pellat/ Rioul ${ }^{5} 2008$ : 411).

7 Selon Massot (2010), dans les énoncés du type c'est pas drôle, la distinction entre la négation de phrase et la négation de constituant est impossible. Toutefois, Klima (1964) fournit une série de tests pour cette distinction (cf. Penka 2011:3-8 pour une discussion). Dans le présent travail, nous ne classons comme négation de constituant que les cas dans lesquels la négation porte indubitablement sur un constituant autre que le noyau du prédicat, comme en (7). 
la phrase matrice il est faux que, nie aussi bien les présuppositions que les enfants et les vitres existent que l'assertion les vitres ont été cassées (cf. Givón 1978: 87-88, Muller 1991: 107-114, Horn ${ }^{2} 2001$ : 21) ${ }^{8}$.

Les deux distinctions suivantes illustrées en (9) et (10), à savoir la négation standard vs. non-standard et syntaxique vs. morphologique, se réfèrent à la description typologique de l'expression de la négation dans les langues du monde.

(9) Négation standard vs. non-standard (selon Miestamo 2005 : 3)

a. Négation standard: le procédé de base et productif d'une langue pour nier les principales verbales

b. Négation non-standard: tous les autres procédés de négation

La négation standard décrit le procédé 'standard', c'est-à-dire le procédé le plus basique et le plus neutre dont une langue dispose pour nier une phrase principale (par exemple, en français, la combinaison de $\pm n e$ et pas).

La définition de négation standard de Miestamo $(2005: 3,44)$ exclut toute construction négative qui ajoute une signification sémantique ou pragmatique en dehors de la négation même ${ }^{9}$. Les adverbes négatifs comme jamais et les pronoms indéfinis négatifs comme personne et rien sont donc exclus de sa définition. Selon Miestamo (2005), le terme de négation standard ne concerne que la négation verbale des principales et des déclaratives.

La négation standard étant une notion typologique, elle n'est pas identique à l'expression de la négation dans la langue standard, qui, elle, fait référence à la catégorie normative du standard linguistique

8 Muller (1991: 107) discute l'exemple suivant: Il ne court pas vite. Ici l'assertion, il court vite (paraphrase: c'est vite qu'il court) est niée, alors que la présupposition il court est maintenue.

9 Ceci exclut la négation associée à d'autres éléments que le verbe conjugué (pour (ne) pas tomber, inattendu) tout comme la négation dans les subordonnées et dans les impératives. Miestamo (2005) exclut également de sa définition tout système de négation qui ne soit ni productif ni général, comme les constructions négatives qui ne s'emploient que dans certains contextes et avec certains verbes. 
tel qu'il est décrit dans les grammaires de référence, comme celle de Grevisse/Goosse ( $\left.{ }^{15} 2011\right)$.

La comparaison de l'expression de la négation standard dans les langues du monde aboutit, généralement, à la distinction de trois types principaux de marqueurs négatifs ( $c f$. Dahl 1979, 2010, Payne 1985 et Dryer $\left.2011 \mathrm{a}^{10}\right)$.

(10) La négation syntaxique vs. morphologique

a. pour lui c'est pas possible (1784)

b. pour lui c'est impossible

Le type de négation standard le plus répandu est l'emploi de particules négatives qui expriment syntaxiquement la négation comme illustré en (10)a. Ce type de négation se trouve dans la moitié des langues du monde, entre autres en français ( \pm ne et pas), en anglais (not), en allemand (nicht) et en latin (non) (cf. Dahl 2010: 19 et section 2.1.3.1). Le deuxième type de négation standard est la négation morphologique ou affixale, utilisée par un tiers des langues comme négation standard des principales (par exemple en turc, $c f$. Bybee 1985 et Miestamo 2005, cités par Dahl 2010: 19) et illustrée en (10)b à l'aide d'un exemple français ${ }^{11}$, même si elle ne constitue pas la négation standard dans cette langue.Enfin, les verbes négatifs constituent le troisième moyen pour exprimer la négation standard et sont utilisés par environ $12 \%$ des langues (par exemple en tongien, $c f$. Dahl 2010: 20-21).

Outre la négation standard, les langues du monde disposent d'une multitude de termes négatifs et 'apparemment négatifs' ( $c f$. Ladusaw

10 La carte interactive de l'atlas mondial des structures linguistiques (World Atlas of Language Structures, WALS, <http://wals.info/index >) correspondant à Dryer 2011a est disponible sous : $<$ http://wals.info/feature/112A?tg_format=map\&v1= $\mathrm{cd} 00 \& \mathrm{v} 2=\mathrm{c} 00 \mathrm{~d} \& \mathrm{v} 3=\mathrm{c} 99 \mathrm{f} \& \mathrm{v} 4=\operatorname{cccc} \& \mathrm{v} 5=\mathrm{c} 909 \& \mathrm{v} 6=\mathrm{cff} 0>$, consulté le 18 juin 2012 .

11 En français moderne, les affixes négatifs servent à l'expression de certaines formes de négation non-standard, comme la négation d'un constituant nominal (la non-réalisation) et la dérivation d'un adjectif (possible $\rightarrow$ impossible). Ces négations ne seront pas considérées dans notre analyse, car il ne s'agit pas de négations de phrase. 
1996: 337 «apparent expressors of negation»), comme fr. personne, it. nessuno ou angl. anybody (appellés mots-n dans la terminologie de Laka 1990). Ces termes manifestent des comportements très divers quant à leur coexistence mutuelle et avec la négation standard: leurs possibilités de cooccurrence sont décrites comme la concordance négative (ou l'association négative dans la terminologie de Muller 1991 et Larrivée 2004).

Généralement, on distingue trois types de comportements principaux: les langues à concordance négative stricte, celles à concordance partielle, donc non-stricte, et celles sans concordance négative.

(11) La concordance négative selon Haspelmath (1997: 201)

\begin{tabular}{|l|l|l|}
\hline $\begin{array}{l}\text { a. Concordance négative } \\
\text { stricte } \\
\text { strict } N C\end{array}$ & $\begin{array}{l}\text { b. Concordance négative non-stricte } \\
\text { non-strict NC (selon la position/ } \\
\text { fonction grammaticale de l'indéfini } \\
\text { négatif dans la phrase) }\end{array}$ & $\begin{array}{l}\text { c. Sans concordance } \\
\text { négative } \\
\text { non-NC }\end{array}$ \\
\hline $\begin{array}{l}\text { français standard, roumain, } \\
\text { polonais, serbo-croate }\end{array}$ & italien, portugais, sarde, espagnol & $\begin{array}{l}\text { allemand standard, } \\
\text { anglais standard, latin }\end{array}$ \\
\hline $\begin{array}{l}\text { français standard } \\
\text { personne n'est venu }\end{array}$ & $\begin{array}{l}\text { italien } \\
\text { a. nessuno viene } \\
\text { b. non vedo nessuno }\end{array}$ & $\begin{array}{l}\text { anglais standard } \\
\text { nobody came }\end{array}$ \\
\hline
\end{tabular}

Dans les langues à concordance négative stricte, comme le français standard, $c f$. (11)a, la coexistence de la particule négative ne et de personne conduit à une interprétation négative simple de la phrase. La concordance non-stricte, par exemple en italien, $c f$. (11)b, est plus complexe: en position préverbale de sujet, nessuno s'emploie sans expression ultérieure de la négation verbale, alors qu'en position postverbale ou d'objet, la présence de la particule de négation est nécessaire. Quant aux langues non-NC, comme l'anglais standard, $c f$. (11)c, celles-ci expriment la négation à l'aide d'un seul élément négatif, et la présence de plusieurs indéfinis négatifs dans une phrase entraîne une interprétation de double négation.

Les types de négation présentés ci-dessus et leurs caractéristiques sont très importants par rapport à l'analyse que nous envisageons, car la présence variable de la particule négative ne dans la négation de 
phrase forme le point central de notre analyse de corpus. Au niveau sémantique, la négation de phrase correspond à la négation interne de l'assertion. En français, elle est toujours exprimée par des moyens syntaxiques, par exemple à travers les particules négatives $\pm n e$ et pas (dans ce cas, la négation de phrase correspond à la négation standard au niveau typologique), ou bien à travers la coprésence de \pm ne et d'un adverbe (jamais, plus) ou d'un indéfini négatif (personne, rien), qui peut être décrite en termes d'une concordance négative. Du point de vue historique, cette coprésence de deux éléments négatifs s'inscrit dans une évolution cyclique, désignée comme cycle de Jespersen.

\subsubsection{L'évolution de la négation en français}

\subsubsection{Le cycle de Jespersen}

Les cycles linguistiques désignent les évolutions au cours desquelles un syntagme ou un élément exprimant une fonction grammaticale disparaît au fil du temps pour être remplacé par une nouvelle unité linguistique ( $c f$. van Gelderen 2009: 2). La réalisation variable de ne, que nous examinerons d'un point de vue synchronique au cours de ce travail, est attribuée diachroniquement à un processus de grammaticalisation cyclique, nommé d'après le linguiste danois Otto Jespersen ${ }^{12}$ ( $c f$. Jespersen 1917, 1924). Ce processus s'est produit dans plusieurs langues indo-européennes (par exemple en anglais et en allemand) et englobe un développement à travers différentes étapes, dont la 'dernière' aboutit de nouveau à 'l'état initial', $c f$. (12) et (13).

12 Otto Jespersen ne fut ni le seul ni le premier à découvrir et à décrire ce phénomène à l'époque. L'égyptologue Alan Gardiner ( $c f$. Gardiner 1904), le linguiste Antoine Meillet ( $c f$. Meillet 1912) et le dialectologue flamand Edgard Blancquaert (cf. Blancquaert 1923) se sont également intéressés à ce genre de changement linguistique. 
(12) Schéma: le cycle de Jespersen (cf. Jespersen 1992[1924]: 479-480, Jäger 2008: 15, van der Auwera 2010: 79)

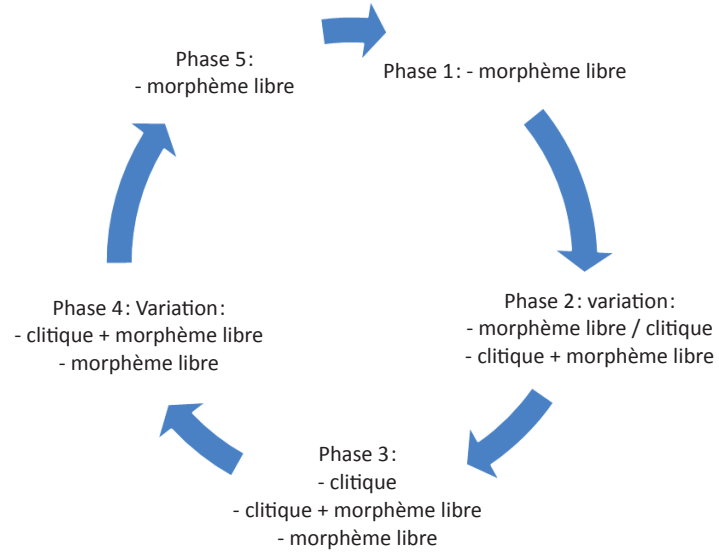

(13) Tableau: le cycle de Jespersen (cf. Jespersen 1992[1924]: 479-480)

\begin{tabular}{|llllll|}
\hline & Phase 1 & Phase 2 & Phase 3 & Phase 4 & Phase 5 \\
\hline Latin/français & $\begin{array}{l}\text { non } \\
\text { ne }\end{array}$ & $\begin{array}{l}\text { ne } \\
\text { ne...pas }\end{array}$ & $\begin{array}{c}\text { ne } \\
\text { ne...pas } \\
\text { pas }\end{array}$ & $\begin{array}{l}\text { ne...pas } \\
\text { pas }\end{array}$ & pas \\
& jeo ne di & & je ne dis pas & je dis pas \\
Allemand & nisagu & & ih ensage niht & ich sage nicht \\
Anglais & ic ne secge & & i ne seye not & i say not
\end{tabular}

Le cycle de Jespersen (notion forgée par Dahl 1979) décrit l'affaiblissement phonétique des éléments négatifs préverbaux qui expriment la négation de façon indépendante au début du cycle $(c f \text {. phase } 1)^{13}$ ainsi

13 Jespersen (1992 [1924]) décrit deux états préliminaires à celui cité ici comme état $1:$ ne dico et non dico. Jespersen (1992 [1924]) ajoute que dans les textes très anciens la première forme, $n e$, apparaît uniquement avec quelques verbes, comme nescio 'je ne sais pas', nequeo 'je ne peux pas' et nolo 'je ne veux pas', et avec certains pronoms et adverbes. Dans tous les autres cas, on trouve la forme latine non, qui remonte à ne renforcé par oenum, 'une chose'; ne-oenum (cf. Jespersen 1992 [1924]: 479). Il est bien possible de voir l'évolution de ne-oenum en non comme un exemple ultérieur (et antérieur) du même cycle de grammaticalisation. 
que la réanalyse et la grammaticalisation des éléments postverbaux. Ces derniers, indiquant initialement de petites quantités ou distances (angl. minimizer, comme PASSUM $>$ pas, MICAM $>$ mie, GUTTAM $>$ goutte et PUNCTUM > point), sont employés par la suite comme des éléments de polarité négative, puis comme des indéfinis négatifs ( $c f$. section 2.1.3.2). Ces éléments, souvent postverbaux, expriment la négation de phrase, d'abord en cooccurrence avec une particule de négation ( $c f$. phase 3 ) et ensuite seuls ( $c f$. phase 5$)^{14}$.

Les états intermédiaires, 2 et 4 dans le cycle sous (13), sont des phases de variation, donc de coexistence de deux ou plusieurs ${ }^{15}$ formes distinctes. Le français moderne se trouve actuellement dans la phase 4: deux formes de l'expression de la négation de phrase, ne...pas et pas, sont en concurrence.

Le rôle de la variation est crucial pour le processus de changement (cf. van der Auwera 2010: 79, Déprez/Martineau 2004, Martineau/ Mougeon 2003, Martineau/Vinet 2005), et son analyse, par exemple à l'aide d'un corpus, comme nous l'envisageons, peut révéler la diffusion et la progression d'un changement à travers le système langagier et la société. Cependant, ce sont exactement ces phases de variation qui sont supprimées dans certaines représentations du cycle de Jespersen qui se limitent à décrire trois états ( $c f$. van der Auwera 2009 pour une discussion).

14 Cf. Breitbarth 2009 pour une nouvelle approche du cycle de Jespersen dans les langues germaniques de l'ouest en termes d'une double réanalyse.

15 Van der Auwera (2010) indique que dans certaines régions de Belgique, où différents dialectes néerlandais se fondent, on trouve jusqu'à cinq structures distinctes en cooccurrence:
a. Ik heb niemand
gezien. (van der Auwera 2010: 99, italiques dans l'original)
b. Ik en heb niemand
c. Ik heb niemand
$\mathrm{d}$. Ik en heb niemand niet gezien.
I NEG have nobody NEG seen
e. Ik heb iemand niet gezien.
I have somebody NEG seen 
Sur le plan historique ${ }^{16}$, les différentes formes d'expression de la négation de phrase en (ancien) français sont résumées dans le tableau (1):

(13) La variation des formes négatives en diachronie (cf. Kawaguchi 2009:207) ${ }^{17}$

\begin{tabular}{|c|c|c|}
\hline Siècle & Forme prédominante & Forme non-prédominante \\
\hline Avant le $9^{e}$ & non... & \\
\hline $9^{\mathrm{e}}-10^{\mathrm{e}}$ & no/ne... & non \\
\hline $11^{\mathrm{e}}-13^{\mathrm{e}}$ & $n e \ldots$ & ne...pas $\leftrightarrow$ ne...mie, ne...point, ne...goutte, non \\
\hline $14^{\mathrm{e}}-15^{\mathrm{e}}$ & $n e \ldots$ & ne...pas $\leftrightarrow n e .$. point, non, ...pas, ...point \\
\hline $16^{\mathrm{e}}$ & ne...pas $\leftrightarrow n e . . . p o i n t$ & ne..., ...pas, ...point, non \\
\hline $17^{\mathrm{e}}-18^{\mathrm{e}}$ & ne...pas & ne...point, ne..., ...pas, ...point \\
\hline$\grave{A}$ partir du $19^{\mathrm{e}}$ & ne...pas & ...pas, ne... \\
\hline
\end{tabular}

À partir du $10^{\mathrm{e}}$ siècle, la particule préverbale ne devient la forme prédominante qui exprime la négation du prédicat sans emphase. Elle est en concurrence avec d'autres formes, car, en effet, jusqu'au $16^{\mathrm{e}}$ siècle, on trouve encore la forme latinisante non. Pendant le Moyen Âge et la période classique, ne s'associe aux éléments postverbaux mie, point, goutte ou pas (éventuellement dans des contextes emphatiques, cf. Grieve-Smith 2010). À partir du $16^{\mathrm{e}}$ siècle, la forme composée ne... pas, illustrée en (14), devient la variante prédominante, et à cette même période, les premières négations sans ne apparaissent, comme l'exemple sous (15) le montre.

(14) Patience, patience; elle ne sera pas toujours jeune (Regnard, Le retour imprévu, 1700, cf. Grieve-Smith 2010: 232)

(15) je penci pas passer le pas

(Les Mazarinades 1641-1652, cf. Martineau/Mougeon 2003: 146)

16 Quant à la chronologie de l'évolution de la négation en français, $c f$. van der Auwera (2010), Dufter/Stark (2007), Grieve-Smith (2010), Kawaguchi (2009), Martineau (2009), Martineau/Mougeon (2003), Martineau/Vinet (2005) et Völker (2003).

17 Le signe $\leftrightarrow$ désigne les variantes en conflit. 
Par la suite, les variantes ne...mie et ne...goutte disparaissent peu à peu, et la variante ne...pas devient celle privilégiée par la norme dans tous les contextes syntaxiques, tout en restant néanmoinsen coexistence avec ...pas jusqu'à nos jours (cf. section 2.2.1.1).

Il est important de noter que, d'un point de vue historique, le processus d'omission de ne n'est pas le même dans les déclaratives que dans les interrogatives, même si les deux phénomènes se ressemblent de manière superficielle ${ }^{18}$. Les contextes interrogatifs et hypothétiques apparaissent déjà sans ne dans les plus anciens textes du français (cf. Martineau 2011), et contrairement aux contextes déclaratifs, l'absence de ne dans les interrogatives est même jugée élégante jusqu'au $17^{\mathrm{e}}$ siècle ( $c f$. Brunot/Bruneau 1949):

(16) L'absence de ne dans les interrogatives VO (cité d'après Martineau 2011: 188-200)

a. Que te semble de ma nouvelle Espousee? Est elle pas bele et honneste souffisamment? (Griseldis, v. 2430-2; Marchello-Nizia 1997: 306)

b. Se bat-on pas toujours quand qu'on devient Cocu? (Brécourt, 1666, Nopce de village)

Dans notre analyse de corpus, l'influence des interrogatives sur la réalisation de ne sera testée ( $c f$. section 4.4.5.2), même si la tendance à l'absence de ne décrite par Martineau (2011) semble s'estomper après le $17^{\mathrm{e}}$ siècle. Néanmoins, il semble possible que le comportement particulier de \pm ne dans les structures à inversion remonte à l'ancien français (cf. la discussion des impératives en section 5.2.4.6).

\subsubsection{Changement rapide ou variation stable?}

Le moment de l'expansion de l'absence du ne de négation n'est pas clairement détectable dans les corpus. Pour la période d'environ 400 ans qui s'étend de 1600 jusqu'à nos jours, deux hypothèses principales existent:

18 Tandis que dans les déclaratives ne est omis en fonction des facteurs (extra) linguistiques, dans les interrogatives avec inversion V-S (i.e. inversion pronominale et complexe), l'absence de la particule est sous-jacente, car il s'agit de contextes de polarité ( $c f$. section 2.1.3.2). 
d'une part, celle d'un changement linguistique rapide et tardif ayant débuté au $19^{\mathrm{e}}$ siècle, comme illustré en (17)a ( $c f$. Martineau/Mougeon 2003), et d' autre part, celle d'un changement lent et continu depuis environ le $17^{\mathrm{e}}$ siècle, illustré en (17)b (cf. Dufter/Stark 2007, Martineau 2011, Poplack/St-Amand 2009).

(17) Changement rapide vs. variation 'stable'

a.

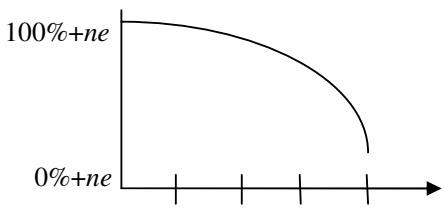

16001700180019002000 b.

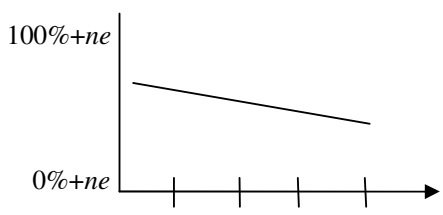

16001700180019002000

Dans la première conception du changement, qui est la plus diffusée dans la littérature au sujet de la variante $\pm n e$, la particule serait réalisée presque sans exception jusqu'au $18^{\mathrm{e}}$ siècle et ne commencerait à disparaître, assez rapidement, qu'en français moderne (cf. Armstrong/ Smith 2002, Ashby 1976, 1981, 2001, Coveney ${ }^{2} 2002$ ) depuis environ le $19^{\text {e }}$ siècle ( $c f$. Martineau/Mougeon 2003). Ce changement récent et rapide aboutirait à la perte totale de ne, comme le montre la chute abrupte de la courbe en (17)a. Ceci signifierait que les occurrences de ne en français moderne sont des vestiges conservés 'artificiellement' par la norme.

Selon le deuxième scénario, la variation $\pm n e$ existe de façon continue et une réalisation absolue de $100 \%$ +ne n'a jamais existé. Dans une telle optique, l'évolution serait éventuellement mieux décrite en termes d'une variation 'stable'. Le changement lent est alors susceptible de stagner et de garder le \pm ne comme une variable à l'intérieur du système linguistique, conditionnée de façon relativement stable par des facteurs (extra)linguistiques.

D'une part, l'idée de la perte totale de ne, illustrée en (17)a, correspondrait aux évolutions de la négation dans d'autres langues et serait en ligne avec les 'prédictions' de Jespersen (1917, 1924). De plus, des études longitudinales semblent soutenir que les taux globaux de 
ne continuent à régresser au $20^{\mathrm{e}}$ siècle (Ashby 2001, Hansen/Malderez 2004, Armstrong/Smith 2002). À ce sujet, Ashby (1981: 686), par exemple, s'attend à une perte totale de ne dans les décennies à venir.

D' autre part, les corpus du $17^{\mathrm{e}}$ siècle manifestent des résultats contradictoires quant à la variation $\pm n e$ ( $c f$. section 2.2.1.1), ce qui pourrait indiquer un changement lent et continu, comme illustré en (17)b. Dans le corpus d'Héroard, (cf. Dufter/Stark 2007, Ernst 1985), le jeune Louis XIII montre déjà au début du $17^{\mathrm{e}}$ siècle, dans un journal transcrit quasi 'phonétiquement' par son médecin Héroard, des régularités de réalisation de ne comparables à celles observables aujourd'hui ( $c f$. section 2.2.1.1), ce qui soutient fortement l'hypothèse d'une variation continue depuis le $17^{\mathrm{e}}$ siècle (cf. (17)b).

Pourtant, un tel patron de variation reste introuvable dans les corpus graphiques de textes littéraires et privés de la même époque (cf. Martineau/Mougeon 2003), car en effet, ceux-ci manifestent une réalisation quasi-catégorique de ne. Pour expliquer cette divergence entre les différents types de corpus, l'on pourrait argumenter que les données d'Héroard sont des manifestations du langage enfantin, qui présente typiquement un pourcentage plus restreint de ne que le langage des adultes ( $c f$. Pohl 1972). Dans cette perspective, l'acquisition de ne serait donc, depuis le $17^{\mathrm{e}}$ siècle, soumise à un processus de gradation d'âge lié à la scolarisation ( $c f$. section 2.2.4.2) ${ }^{19}$.

Toutefois, cette explication serait trop simple, car les études longitudinales d'Armstrong/Smith (2002), d'Ashby (2001) et d'Hansen/ Malderez (2004) ont montré que du moins les locuteurs adultes d'aujourd'hui ne rajustent plus autant que leurs parents leur production langagière en direction de la norme. De plus, la particule ne n'est pas strictement absente du langage des enfants, ce qui signifie qu'il ne s'agit donc pas d'un 'défaut' enfantin dû à l'acquisition précoce et incomplète

19 Ce décalage est généralement nivelé au cours de l'acquisition du langage et de l'éducation scolaire (angl. age grading). Paradoxalement, dans le langage du Dauphin, nous observons une tendance opposée: l'omission de ne devient plus fréquente entre 5 et 9 ans par rapport à la période précédente. Le langage enfantin ne représente donc aucune explication globale des disparités entre les deux corpus (comme le remarquent aussi Dufter/Stark 2007 et Martineau 2009). 
de la morphosyntaxe ( $c f$. section 2.2.4.2). Par conséquent, même si la gradation d'âge semble jouer un rôle, elle ne peut être le seul facteur qui distingue le journal d'Héroard d'autres corpus de cette époque.

Martineau (2009: 167-168) propose une approche plus convaincante pour expliquer les divergences entre le journal d'Héroard et les autres corpus (de textes) contemporains:

(18) For dialogic texts, the low frequency of deletion of ne may partly be explained by the fact that the absence of $n e$ was not a salient feature at that time. ${ }^{20}$ [...] If the deletion of $n e$ was not socially salient enough at that time, authors may have chosen not to use it. (Martineau 2009: 167-168)

Le raisonnement de Martineau (2009, réaffirmé dans Martineau 2011 : 202-203) signifie que les auteurs des pièces de théâtre populaires de l'époque essayaient, en guise d'authenticité, d'introduire des marques (angl. features) linguistiques du langage populaire dans leurs textes. N'étant pas une marque communément reconnue (angl. salient feature) du langage populaire à l'époque, l'omission du ne était ignorée dans la conception des dialogues. Autrement dit, si la non-réalisation de ne passait largement inaperçue au $17^{\mathrm{e}}$ siècle, le fait de sa présence graphique dans les journaux intimes et les pièces de théâtres ne reflèterait pas la réalisation phonique de l'époque. Dans ce cas, la variation $\pm n e$ serait plus ancienne que sa documentation dans les corpus graphiques du $19^{\mathrm{e}}$ siècle.

D’une manière générale, même si une datation exacte du début du changement linguistique reste impossible, il semble clair que cette question est cruciale pour l'évaluation de la variation $\pm n e$ observée actuellement en français moderne. Il est possible d'affirmer que plus l'absence de ne remonte dans le temps, plus le changement doit être conçu comme une évolution lente. Si le changement est lent et continu, comme le suggèrent les données du corpus d'Héroard ( $c f$. Dufter/Stark 2007 et section 2.2.1.1), il s'agit donc plutôt d'une variation 'stable' et une perte totale de ne en français moderne semble lointaine ( $c f$. section 5.4.3 pour une discussion de cette question à la lumière des données de corpus).

20 Martineau (2009: 167-168) remercie Antony Lodge pour cette suggestion. 


\subsubsection{La négation en français moderne}

\subsubsection{Les particules négatives ne et pas}

Les particules négatives sont des termes négatifs indépendants et non fléchis qui ne véhiculent aucune signification autre que celle de la négation ( $c f$. Dahl 2010: 19). Pas, la principale particule négative du français moderne (souvent décrite comme une sorte d'adverbe, $c f$. par exemple Jones ${ }^{3} 2007$ ), suit le verbe fléchi dans les phrases tensées ${ }^{21}$, mais peut en être séparée par certains adverbes, comme par exemple absolument, toujours ou malheureusement:

(19) La syntaxe de pas

a. je peux pas lire sans ma feuille tu vois (1924)

b. je peux absolument pas ouvrir l'œil (C-ORAL-ROM)

Par contre, l'élément ne est une particule proclitique, ce qui signifie que (si réalisée) elle est antéposée à une base lexicale, en français c'est leverbe fléchi, $c f$. (20)a. De cette base la particule ne peut être séparée que par d'autres proclitiques, comme par exemple le clitique réfléchi se, $c f$. (20)b.
(20) La syntaxe de ne
a. je ne sais pas (1463)
b. le dialogue ne se joue que pour le lecteur (0050)
c. je (*au contraire) ne sais pas
d. je ne (*absolument) sais pas
e. la monogamie obligatoire / au contraire / ne s'observe que dans les socié- tés les plus élevées (C-ORAL-ROM)

L'introduction du matériel lexical non-clitique, comme l'apposition au contraire en (20)c ou l'adverbe absolument en (20)d, dans la chaîne des proclitiques est impossible. En revanche, derrière un sujet lexical nonclitique, comme la monogamie obligatoire en (20)d, l'insertion d'une

21 Par contre, dans les constructions infinitivales, qui ne seront pas considérés dans notre analyse ( $c f$. section 2.1.1), les deux particules ne (si réalisée) et pas précèdent l'infinitif du verbe: je suis pas d'accord sur ne pas s'exprimer là-dessus (C-ORAL-ROM). 
apposition avant ne est possible. Les proclitiques forment donc une séquence de clitiques (angl. clitic cluster) à l'intérieur de laquelle l'ordre et les possibilités de cooccurrence suivent des règles grammaticales particulaires ( $c f$. section 2.2.5.3).

Même si ne est généralement conçu comme une particule négative, ni son statut négatif ni celui de particule sont indubitablement prouvés. Premièrement, même si ne seul peut exprimer la négation de phrase ${ }^{22}$ dans certaines constructions figées, il connaît également des emplois non-négatifs comme ne-explétif ${ }^{23}$. Deuxièmement, la position de Dahl (2010: 19), qui considère que les particules sont 'indépendantes', n'est pas valable pour le ne français. En effet, les clitiques étant des unités à mi-chemin entre les morphèmes libres et les affixes ( $c f$. Zwicky 1977: 1 «neither clearly independent words, nor clearly affixes»), ils ne répondent donc ni à la catégorie d'une particule 'indépendante' ni à celle des affixes négatifs. La forte union entre ne et le verbe fléchi pourrait faire penser qu'il s'agit plutôt d'un affixe que d'un clitique ( $c f$. l'approche de Culbertson 2010 discutée en section 2.3.3). Malgré ces problèmes de classification, ne sera désigné, au cours du présent travail, de manière théoriquement neutre, comme particule négative ou comme clitique négatif.

$\mathrm{Au}$ niveau syntaxique, il existe trois analyses principales de la négation de phrase en français. Premièrement, l'analyse 'standard' en termes d'un syntagme négatif, désigné comme NegP ( $c f$. Rowlett 1998 basé sur Pollock 1989 et Haegeman 21996). Deuxièmement, une analyse

22 Ne peut exprimer la négation de phrase avec certains verbes, par exemple cesser, oser et pouvoir, cf. Elle n'osa tourner la tête (France, Histoire comique, XVI, cité par Grevisse/Goosse ${ }^{15} 2011$ : 1276).

23 Le ne-explétif s'emploie avec un terme exprimant (a) l'inquiétude, l'interdiction ou l'évitement de justesse: Pierre a toujours peur que Marie ne s'en aille; (b) le doute ou la contestation en construction négative ou interrogative: Marie ne doute pas que Pierre ne l'aime. Marie a-t-elle jamais nié que Pierre ne l'ait aimée?; (c) après les conjonctions de subordination avant que, à moins que et (récemment aussi) sans que: Marie est partie avant que/sans que Pierre n'ait esquissé un geste pour la retenir; (d) dans les constructions comparatives: Pierre aime sa fille plus/moins que Marie ne l'aime (cf. Wilmet 2007: 228-232). 
de l'accord négatif selon Peters (1999) (dans le cadre du programme minimaliste $c f$. Chomsky 1995), qui suppose que la particule négative pas porte une marque négative interprétable [i-neg] en accord avec une propriété négative non-interprétable [u-neg] du verbe ( $c f$. Remberger 2006: 99-100 pour une analyse comparable de l'italien). Troisièmement, Déprez (2003) et Roberts (2007) analysent (dans une version plus récente du programme minimaliste, $c f$. Chomsky 2000, 2001) la cooccurrence de plusieurs termes négatifs en français (et dans d'autres langues et variétés) comme accord négatif ( $c f$. la notion de concordance négative en section 2.1.1 ainsi que Biberauer/Roberts 2011 et Zeijlstra 2004).

Notre analyse de corpus envisage la présence variable de la particule ne de manière empirique et reste donc théoriquement neutre en termes de modèles syntaxiques. Pour des raisons de lisibilité, nous adaptons, dans les cas où une modélisation syntaxique est nécessaire, l'analyse 'classique' de la négation développée par Rowlett (1998) suivant Pollock (1989):

(21) NegP selon Rowlett (1998: 20)

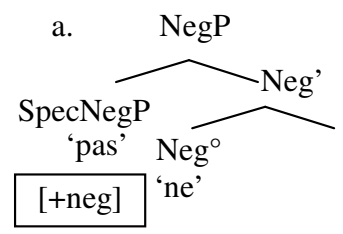

Ce modèle d'analyse soutient que ne est la tête de NegP, qui est cliticisé à gauche d'un verbe fléchi, tandis que pas, doté d'une propriété négative [+neg], se place dans la position de spécificateur du syntagme. Comme la plupart des analyses syntaxiques de la négation en français, le modèle de Rowlett (1998) se base sur le français standard, où la négation de phrase est exprimée par ne et pas $^{24}$.

24 L'analyse minimaliste de Peters (1999) constitue une exception à cette généralisation. Cette analyse en termes d'accord entre une marque négative noninterprétable du verbe [u-neg] et interprétable de pas [i-neg] met la particule pas au centre de l'opération syntcatico-sémantique de négation, tandis que ne (si réalisé) est conçu comme tête sémantiquement vide en T qui est cliticisée sur PF. 
$\mathrm{Vu}$ que ce modèle accorde une position de tête, donc une position indispensable, à ne, les cas où la particule ne est absente peuvent être analysés en admettant que celle-ci est présente en syntaxe mais effacée en phonologie.

Par conséquent, et contrairement à l'opinion de Koch/Oesterreicher ( 22011 : 25), l'intégration de l'absence et la présence variable de ne dans une approche générative est généralement possible, comme le notent également Coveney (2002: 35), Dubois (1967: 137) et Jones $\left({ }^{3} 2007\right.$ : 348). Cependant, la question de base demeure: quelles sont les régularités qui gouvernent cette variation? L'analyse de corpus au chapitre 4 permettra de discerner les facteurs pertinents pour \pm ne et éventuellement aussi de formuler des règles pour sa présence variable ( $c f$. la discussion en chapitre 5).

\subsubsection{Les termes négatifs et de polarité}

Outre les particules négatives, que nous avons présentées dans la section précédente, d'autres éléments apparaissent dans les contextes négatifs, comme nous l'avons vu lors de la brève présentation de la concordance négative dans la section 2.1.1.

Dans les langues du monde, deux autres groupes de termes contribuent à l'expression de la négation. Il s'agit, d'une part, des pronoms, des déterminants et des adverbes négatifs, $c f$. 1 . en (22), et, d'autre part, des termes de polarité, cf. 2. en (22). Tandis que les éléments en (22)1 expriment la négation de phrase, tout comme les particules négatives (cf. Jäger 2008: 15, de Swart 2010: 11 et FN 5), ceux en (22)2 dénotent les extrémités d'échelles pragmatiques contextuellement déterminées.

Pour l'analyse de corpus envisagée dans ce travail, ce sera le premier groupe, à savoir les termes négatifs, qui seront pris en considération. Le deuxième groupe, c'est-à-dire les termes de polarité, ne sont présentés ici que pour des raisons d'intégralité et dans le but de pouvoir les distinguer nettement des termes négatifs. La distinction est particulièrement délicate en français, puisque certains éléments, comme par exemple personne, jamais et rien, apparaissent dans les deux groupes. 
(22) Les termes négatifs et de polarité ( $c f$. Werle 2002: 1, légèrement modifié25)

\begin{tabular}{|c|c|c|c|c|c|c|}
\hline & & & Français & Anglais & Espagnol & Russe \\
\hline 1. a. & $\begin{array}{l}\text { Pronoms indéfinis et dé } \\
\text { négatifs (PIN et DN) }\end{array}$ & rminants & personne, nul & no & - & - \\
\hline b. & Adverbes négatifs (Ad & & jamais $^{26}$ & never & nunca & nikogda \\
\hline 2. c. & $\begin{array}{l}\text { Termes de polarité } \\
\text { négative (TPN) }\end{array}$ & $\begin{array}{l}\text { Termes } \\
\text { de }\end{array}$ & $\begin{array}{l}\text { quoi que ce } \\
\text { soit }\end{array}$ & any & ningún & $\begin{array}{l}\text { kakoj- } \\
\text { libo }\end{array}$ \\
\hline d. & $\begin{array}{l}\text { Termes de libre choix } \\
\text { (TLC) }\end{array}$ & polarité & rien $^{27}$ & & cualquier & ljuboj \\
\hline
\end{tabular}

a. Les pronoms indéfinis et les déterminants négatifs ( $c f$. angl. série de no: no, nothing, no one/nobody) sont à décrire comme des quantifieurs universels ou existentiels sous la portée d'une négation. Ils combinent la quantification avec une négation (cf. Stark 2006: 191 et section 2.1.1) et expriment une valeur négative en isolement (par exemple: Who came? Nobody). Selon Haspelmath (1997), ces termes (tout comme leurs équivalents positifs, angl. something, esp. algo etc.) se caractérisent du point de vue formel par le fait d'être des pronoms au sens large, donc de pouvoir remplacer des syntagmes déterminatifs (DP) dans une phrase, et du point de vue fonctionnel par le fait d'exprimer la référence indéfinie ( $c f$. Haspelmath 1997: 10-11) ${ }^{28}$. Ils

25 Nous ajoutons le français dans le tableau et supprimons la classe des termes de concordance négative (TCN: negative concord items), qui constitue une sousclasse des TPN restreinte à la négation directe et toujours en cooccurrence avec la particule de négation (cf. russe, série de nikakoj: nichto, nikto, nigde, nikogda).

26 Employé dans les propositions simples.

27 Employé dans les contextes interrogatifs, hypothétiques ou de comparaison.

28 La distinction entre un syntagme déterminatif comme no man et un pronom indéfini comme nobody n'est pas toujours évidented'un point de vue synchronique. Comme critères de distinction, Haspelmath (1997: 10) cite la flexibilité syntaxique (no single man vs. *nosinglebody) et la variabilité morphologique (*nothings vs. no things), notant que la phonologie est parfois la seule façon de faire la distinction entre un pronom indéfini (nothing ['n $\Lambda \theta \mathrm{I} y]$ ) et un syntagme

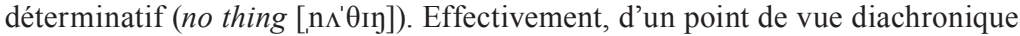
un lien étroit entre les deux éléments: les pronoms indéfinis dérivent souvent de syntagmes déterminatifs lexicalisés. 
n'ont besoin d'aucune association négative avec d'autres négations ( $c f$. licensing, Ladusaw 1996), et par ailleurs, en combinaison avec la négation de phrase ou avec un autre quantifieur négatif, ils provoquent une lecture de négation multiple (cf. (23)).

(23) Sans concordance négative (non-NC): l'anglais standard

a. No one saw nothing 'personne (n)'a rien vu'

(Werle 2002: 2)

b. Formule logique: $\neg \exists \mathrm{x} . \neg \exists \mathrm{y} .[\operatorname{personne}(\mathrm{x}) \& \operatorname{chose}(\mathrm{y}) \&$ a $v u(\mathrm{y})(\mathrm{x})]$

c. Paraphrase: Il n'y a aucune personne $x$ de sorte qu'il n'y ait aucune chose $y$ de sorte que $x$ ait vu $y$.

$\Rightarrow \quad$ En d'autres termes, au moins une personne a vu quelque chose.

Nous avons vu en (23) qu'en anglais standard, l'occurrence d'un seul élément de la série de no est suffisante pour donner une interprétation négative à la phrase. La combinaison de deux éléments de cette série donne lieu à une double négation ${ }^{29}$ (en logique $\neg \neg p=p$ ). Il s'agit donc d'une langue sans concordance négative ( $c f$. section 2.1.1).

En comparaison avec l'anglais, la situation des termes négatifs en français moderne est assez complexe. Premièrement, les pronoms et déterminants négatifs n'y forment aucune série morphologiquement reconnaissable et sont à définir en fonction de critères purement syntaxiques et sémantiques. En accord avec Jäger (2008: 21) et de Swart (2010: 11), et contrairement à Muller (1991), Riegel/Pellat/Rioul (52008) et Larrivée (2004), nous classons personne et rien comme des pronoms indéfinis négatifs et aucun $\mathrm{N} /$ mul $\mathrm{N}$ comme des déterminants négatifs, car ces syntagmes peuvent occuper une position d'argument (sujet/ objet) dans la phrase négative, comme en (24), et exprimer ainsi une valeur négative dans les réponses elliptiques, $c f$. (25).

29 Toutefois, en anglais non-standard (et ceci vaut également pour l'allemand nonstandard), la construction en (23)a est ambigüe, car la possibilité d'une deuxième lecture comme négation simple ('personne n'a rien vu') existe. Nous nous limitons ici à mentionner cette possible ambigüité et gardons l'anglais standard comme exemple de langue non-NC, c'est-à-dire de langue dans laquelle plusieurs quantifieurs négatifs s'excluent mutuellement. 
(24) Les PIN et DN exprimant la négation de phrase en fonction de sujet et d'objet
a. personne ne s'est mîu
b. vous avez vu personne ici en haut ce matin?
c. j'attends / et rien ne se passe
d. mardi elle nous a rien donné
e. êtres supérieurs qui les succéderaient un jour / qu'aucun savant philo- sophe ni poète n'a jamais imaginés surtout sous les [?] singes

f. Ulysse ne nous montre aucun intérêt dans le dialogue

(25) Les PIN et DN comme réponse elliptique. Qui as-tu vu?

a. personne

b. rien

c. aucun/nul homme

L'on pourrait se demander pourquoi personne et rien, qui peuvent évidemment apparaître en cooccurrence avec ne, sont classés comme PIN, c'est-à-dire en analogie avec angl. nobody et nothing, et non pas comme TPN, en analogie avec angl. anybody et anything.

Mathieu (2001 : 320) démontre, en se basant sur de nombreux tests syntaxiques, que les PIN français personne et rien apparaissent souvent en distribution complémentaire avec les TPN et que ceux-ci montrent donc plutôt des similitudes avec les PIN anglais nobody et nothing. Outre la possibilité d'exprimer la négation dans les réponses fragmentaires négatives, dont anybody et anything sont privés, Mathieu (2001) mentionne la possibilité d'une lecture de négation multiple lorsque personne et rien sont en cooccurrence, comme c'est le cas en allemand et en anglais standard, $c f$. (26).

(26) Les PIN permettent une lecture de double négation

Personne a rien dit.

(cf. Mathieu 2001 : 331-332)

'No one said anything.'/ 'No one said nothing'

(i) 'There are no $\mathrm{x}$ and no $\mathrm{y}$, such that $\mathrm{x}$ is a person, and $\mathrm{y}$ is a thing, and $\mathrm{x}$ said y.' (concordance négative)

(ii) 'It is not the case that there are no $\mathrm{x}$ and no $\mathrm{y}$, such that $\mathrm{x}$ is a person, and $\mathrm{y}$ is a thing, and $\mathrm{x}$ said $\mathrm{y}$.' (double négation) 
Le fait que deux lectures de l'exemple (26) soient disponibles prouve que personne, rien, aucun $N$ et nul $N$ sont des pronoms et des déterminants négatifs. Il existe donc une lecture (i) de concordance négative (possible en anglais et en allemand non-standard) ainsi qu'une lecture (ii) de double négation (comme dans les langues non-NC, l'anglais et l'allemand standard). Par ailleurs, personne et rien connaissent également des emplois non-négatifs comme terme de libre choix ( $c f$. $\mathrm{d}$. en bas).

b. Les adverbes négatifs ( $\mathbf{A d v N})$ peuvent exprimer la négation inhérente (cf. angl. nowhere, never), comme les PIN et DetN, en ajoutant une nuance sémantique à la négation. Pour cette raison, Werle (2002) les classe avec les PIN et DetN comme des quantifieurs négatifs. En français, les adverbes négatifs jamais, plus et guère s'opposent ${ }^{30}$ sémantiquement à toujours, encore et très bien/beaucoup. Les contreparties positives de nulle part sont des syntagmes prépositionnels comme dans le jardin ou les adverbes locatifs comme ici ou là. Nullement et aucunement expriment une intensification de la négation, tout comme l'ajout $d u$ tout au négateur standard $\pm n e . . . p a s$.

La plupart des AdvN (à l'exception de plus) s'emploit comme des réponses elliptiques négatives, $c f$. (27).

(27) Les AdvN comme réponse négative elliptique

a. Es-tu d'accord? - Nullement/aucunement/jamais/*plus.

b. Tu les as trouvés? - Jamais/nulle part/*plus.

Il existe bien sûr également des occurrences purement positives de plus en français moderne. Cette ambiguïté peut être aussi observée dans notre corpus:

(28) L'ambiguité de plus

a. je suis sûre qu'y en aurait plus (2812/2814)

b. [зәsцізувkijã noвєplys] 'je suis sûre qu'il y en aurait davantage' [-neg] $(2812 / 2814)$

c. [зәsцізувkijã noвєрly] 'je suis sûre qu'il y en aurait pas’ [+neg]

30 Muller (1991: 138) décrit (ne)... plus comme «l'amalgame d'une négation et d'un morphème signifiant encore». 
Dans la transcription orthographique, aussi bien la lecture positive de plus en (28)b que celle négative en (28)c seraient admises. La transcription phonétique, par contre, permet une désambiguïsation grâce à la consonne finale $[\mathrm{s}$ ] ( $c f$. section 3.7 pour le système de la double transcription). Dans l'exemple (28)a, tiré de notre corpus, l'occurrence de plus avec un [s] final,signifie davantage, tandis que plus, prononcé sans [s] final, comme illustré en (28)c, exprime une négation de phrase. Les cas comme les énoncés en (28)b, dans lesquels plus est interprétable comme davantage, seront évidemment exclus de l'analyse de corpus.

Pour la plupart des termes négatifs, la lecture positive n'est plus disponible ou bien un clivage lexical a eu lieu (cf. lexical split, Roberts/Roussou 1999: 1025). Dans ce cas, il s'agit de deux éléments lexicaux distincts qui ne sont plus interchangeables, comme par exemple pas, la particule négative, et un pas, le syntagme déterminatif. Les emplois positifs de plus ainsi que les emplois positifs de personne et rien dans l'exemple (38) témoignent de la complexité sémantique et syntaxique des termes négatifs en français moderne, qui dérivent presque sans exception de termes positifs ( $c f$. la vue d'ensemble des étymologies des termes négatifs en français moderne en tableau (39)).

Enfin, avant de clore cette section consacrée aux termes négatifs, il ne reste plus qu'à mentionner deux autres éléments qui entrent dans le contexte de la négation de phrase en français et qui sont donc pertinents pour l'analyse de corpus. Il s'agit, d'abord, du terme restrictif que, qui montre une certaine similitude avec les termes négatifs en raison de sa possible cooccurrence avec ne. Cependant, d'un point de vue sémantique, que n'exprime pas la négation mais l'affirmation sous certaines conditions, c'est-à-dire que les énoncés qui contiennent que peuvent être reformulés à l'aide de l'adverbe seulement, $c f$. (29).

(29) Le terme restrictif que

a. le dialogue ne se joue que pour le lecteur

b. le dialogue se joue seulement pour le lecteur 
Suivant la méthode d'Armstrong/Smith (2002), Ashby (1976, 1981, 2001), Coveney (2002) et Hansen/Malderez (2004), entre autres, l'élément restrictif que sera inclus dans l'analyse de corpus en raison de sa cooccurrence avec la variable $\pm n e$.

Le second élément pertinent est la conjonction de coordination négative $n i$ (ou $n i . . . n i$ ), qui apparaît, elle aussi, en cooccurrence avec $\pm n e$ (aussi avec sans, cf. Grevisse/Goosse ${ }^{15} 2011$ : §1087):

(30) La conjonction de coordination négative $n i \ldots n i$

a. êtres supérieurs qui les succéderaient un jour /qu'aucun savant philosophe ni poète n'a jamais imaginés surtout sous les [?] singes (0088/0090)

b. je l'apparenterais ni à un savant peut-être pas non plus à un homme d'église (0025)

Considérons maintenant le deuxième groupe d'éléments présentés dans le tableau sous (22), à savoir les termes de polarité. Les termes de polaritésont des indéfinis scalaires, utilisés pour exprimer la nonspécificité ou une valeur négative, sans pourtant comporter la négation de manière inhérente. Ils ont besoin de la présence d'un déclencheur (angl. trigger) sémantique, comme par exemple une négation ( $c f$. aussi les contextes de polarité négative en (35)). Contrairement aux termes négatifs, dont l'expression de la négation est inhérente, les termes de polarité (ou indéfinis scalaires) développent leur capacité à exprimer la négation ou la référence indéfinie selon les contextes où ils se trouvent.

Le principe d'implication scalaire en (31) est à la base de toute analyse des termes de polarité ( $c f$. Fauconnier 1975, Krifka 1995, Haspelmath 1997, Werle 2002).

(31) Le principe d'implication scalaire selon Fauconnier (1975: 193)

a. Scale principle: If $\mathrm{x}_{1}$ is lower than $\mathrm{x}_{2}$ on the scale $\mathrm{S}$, than $\mathrm{R}\left(\mathrm{x}_{1}\right)$ implicates $\mathrm{R}\left(\mathrm{x}_{2}\right)$.

b. Corollary: if R holds for the lowest element on S, it holds (by implicature) for all elements 
(32) L'échelle pragmatique et les termes de polarité

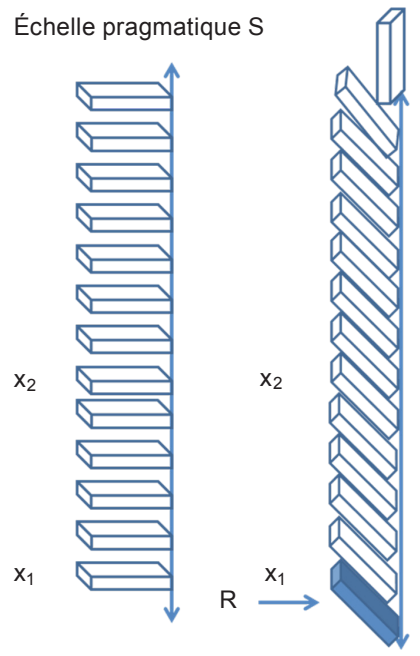

L'échelle pragmatique en (32) nous permet de décomposer la sémantique des termes de polarité. Pour illustrer ce mécanisme, considérons l'expression figée ne pas lever le petit doigt ${ }^{31}$, qui dénote, comme les termes de polarité indéfinis (cualquier, anybody etc.), l'extrémité d'une échelle pragmatique. Dans la paire minimale présentée en (33), cette expression s'emploie avec la négation de phrase, comme en (33)a, mais est agrammaticale dans l'assertive correspondante en (33)b.

(33) L'emploi des termes de polarité (cf. Muller 1991: 71)

a. Jean n'a pas levé le petit doigt pour nous aider.

b. *Jean a levé le petit doigt pour nous aider.

L'expression figée lever le petit doigt dénote le moindre mouvement possible pouvant être entrepris (par exemple pour aider quelqu'un). Ce mouvement constitue donc $\left(\mathrm{x}_{1}\right)$, l'extrémité inférieure de l'échelle

31 D'autres expressions figées du français peuvent être analysées de la même manière: ne pas être tombé de la dernière pluie, ne pas faire de mal à une mouche, ne pas avoir inventé l'eau chaude (cf. Muller 1991 : 71). 
pragmatique en (32), et c'est pour cette raison qu'on appelle ces expressions polaires minimizers. Tous les autres mouvements (de l'index, de la main, du bras, des jambes, du corps entier $=x_{2}, x_{3}, x_{4}$ etc.), sans doute plus utiles pour aider quelqu'un, seraient plus couteux que le mouvement du petit doigt. L'action minimale pour aider quelqu'un est donc le mouvement du petit doigt, et par implicature scalaire, si ce mouvement n'est pas entrepris, aucun mouvement ne pourra l'être. Selon Fauconnier (1975), ce qui vaut pour l'élément à l'extrémité inférieure de l'échelle, vaut également par implicature pour tous les autres éléments de l'échelle.

c. Les termes de polarité négative (TPN) (angl. negative polarity items) sont des indéfinis restreints à la négation directe ou aux contextes de polarité négative (CPN) qui, de façon isolée, donnent lieu à une interprétation négative (esp. nada, nadie, en ninguna parte, nunca). Hoeksema (2000: 115) les définit de manière suivante:

(34) Negative polarity items are expressions (either words or idiomatic phrases) with a limited distribution, part of which always includes negative sentences. Simplifying a bit, we can say that negative polarity items are items that give rise to minimal pairs of affirmative and negative sentences, of which only the negative member is grammatical.

Les contextes de polarité négative $(\mathrm{CPN})^{32}$ (angl. triggers), c'est-à-dire les contextes qui permettent l'occurrence des TPN indéfinis ou figés, ne se limitent pas aux contextes négatifs morphologiquement transparents comme $\pm n e . . . p a s$, mais comprennent aussi les adverbes non proprement négatifs comme rarement et les prédications comme douter ou improbable. Les CPN sont également employés dans les phrases hypo-

$32 \mathrm{Vu}$ la vaste gamme de constructions syntaxiques qui peuvent être $\mathrm{CPN}$, un critère syntaxique qui les unifie semble difficile à trouver. Pour éviter ce problème, Ladusaw (1996), suivant Fauconnier (1975), propose un critère sémantique: les CPN permettent tous l'implication descendante (angl. downward entailment cf. Ladusaw 1996: 328, all. abwärts-monoton cf. Eckardt 2003: 24) et le renversent de la polarité d'une proposition, $c f$. Hoeksema (2000), Krifka (1995: 209-218) et Ladusaw (1996: 328) pour une discussion. 
thétiques et dans les interrogations totales, comme l'observe Ladusaw (1996: 327):

(35) Les contextes de polarité négative

a. Jean lève rarement un doigt pour nous aider.

b. Je doute que Jean va lever un doigt pour nous aider.

c. Si Jean lève un doigt pour nous aider, cela m'étonnerait.

d. Jean a-t-il levé un doigt pour nous aider?

En français, le groupe des TPN comprend des indéfinis comme quiconque ou un/une $\mathrm{N}$ quelconque et des expressions plus complexes dénotant les quantités minimes ( $c f$. le/la moindre $\mathrm{N}$ que ce soit/lever le petit doigt), la non-spécificité (cf. qui que ce soit/quoi que ce soit) ou une caractéristique très générale du référent, comme [+animé] ( $c f$. il n'y a pas âme qui vive).

d. Les termes de libre choix (TLC) (angl. free choice items) sont des indéfinis restreints aux fonctions non-spécifiques (selon Haspelmath 1997) qui n'expriment aucune négation (cf. esp. cualquier, cualquier cosa, cualquier persona etc. $)^{33}$. En espagnol, il s'agit de la série de cualquier (cualquier cosa, cualquier persona etc.), ce qui signifique que dans l'exemple espagnol en (36)a, il est obligatoire d'utiliser cualquier, afin de générer la même interprétation que any dans l'exemple (36)b. Par contre, l'emploi de ninguna serait agrammatical dans la lecture non-spécifique.

(36) L'emploi des termes de polarité (cf. Werle 2002: 15)

a. Cualquier/*ninguna lechuza caza ratones. (lecture non spécifique)

'N'importe quelle/aucune chouette chasse les souris'

b. Any owl hunts mice.

33 Haspelmath (1997) explique la différence entre une lecture spécifique et nonspécifique à l'aide de l'exemple suivant: Nobuko wants to marry a native speaker of Аinu. Pour en faire une lecture spécifique, la phrase pourrait se poursuivre de la façon suivante: ... She fell in love with him during fieldwork sessions. Par contre, si la phrase se poursuit par ...because she is Ainu herself, and she wants her children to acquire her ancestors' language, la lecture de a native speaker of Ainu est non-spécifique (cf. Haspelmath 1997: 37). 
En français, la classe des TLC coïncide entièrement avec celles des PIN et des AdvN: les PIN personne et rien et l'AdvN jamais s'emploient en tant que négations propres (PIN et $\mathrm{AdvN}$ ) et en tant que TLCs. Dans les propositions simples, personne, rien et jamais véhiculent la négation inhérente, tandis qu'ils s'emploient aussi, dans certains cas plutôt rares mais existants depuis le Moyen Âge jusqu'à aujourd'hui, comme des termes de libre choix (TLC) sans valeur négative. Ces emplois concernent les contextes interrogatifs, hypothétiques ou de comparaison. Dans ces contextes-là, les TLC sont remplaçables en français par des pronoms indéfinis positifs comme quelqu'un et se traduisent en anglais par anybody, anything ou ever:

(37) L'emploi non-négatif de rien au $17^{\mathrm{e}}$ siècle ( $c f$. Martineau 2011: 188-200) As-tu rien vu de plus joli? (Molière, 1665, Dom Juan, Acte 2, scène 3)

(38) Les emplois non-négatifs de personne, rien et jamais en français moderne (cf. Wilmet $\left.{ }^{2} 1998: 274\right)^{34}$

b. As-tu vu personne (/quoi que ce soit/quelque chose)?

'Have you seen anybody?'

c. Savez-vous rien (/quoi que ce soit/quelque chose) de Pierre?

'Do you know anything about Peter?'

d. As-tu jamais (/déjà) visité Paris?

'Have you ever visited Paris?'

Les occurrences de personne, rien et jamais dans les contextes interrogatifs, hypothétiques ou de comparaison ne seront donc pas considérées dans notre analyse de corpus, puisqu'il ne s'agit pas de négations de phrase.

Pour conclure cette section, le tableau en (39) résume les termes négatifs et de polarité du français moderne et précise leur étymologie (souvent positive).

34 D'autres exemples seraient la phrase hypothétique Si Pierre devait aimer rien/ quoi que ce soit/quelque chose/n'importe quoi plus que Marie et la comparaison Pierre aime Marie plus que personne/quoi que ce soit/*quelque chose/n'importe quoi (cf. Wilmet ${ }^{2} 1998$ : 274). 
(39) Les termes négatifs (en blanc) et de polarité (en gris) en français moderne ${ }^{35}$

\begin{tabular}{|c|c|c|c|c|c|c|}
\hline $\begin{array}{l}\text { Pronoms } \\
\text { indéfinis et } \\
\text { déterminants } \\
\text { négatifs }\end{array}$ & $\begin{array}{l}\text { personne } \\
<\text { PERSONAM }\end{array}$ & $\begin{array}{l}\text { rien lat. } \\
<\text { REM 'chose' }\end{array}$ & $\begin{array}{l}\text { aucun(e) } \mathrm{N} \\
*_{\text {ALICUNU/ }} \\
\text { ALIQUEM } \\
\text { UNUM }\end{array}$ & $\begin{array}{l}n u l \mathrm{~N} \\
<\text { NULLUM }\end{array}$ & & \\
\hline $\begin{array}{l}\text { Adverbes } \\
\text { négatifs }\end{array}$ & $\begin{array}{l}\text { aucunement } \\
<* \text { ALICUNU/ } \\
\text { ALIQUEM } \\
\text { UNUM }\end{array}$ & $\begin{array}{l}\text { nullement } \\
<\text { NULLUM }\end{array}$ & $\begin{array}{l}\text { jamais } \\
\text { (<afr. jà } \\
\text { 'davantage' } \\
+ \text { mais } \\
\text { 'plus') }\end{array}$ & \begin{tabular}{|l|} 
nulle part \\
$<$ NULLUM \\
PARTEM
\end{tabular} & $\begin{array}{l}\text { plus (lat. } \\
<\text { PLus } \\
\text { 'davantage' }\end{array}$ & \begin{tabular}{|l|} 
guère \\
<francique: \\
waigaro \\
'beaucoup'
\end{tabular} \\
\hline $\begin{array}{l}\text { Terme de } \\
\text { Restriction }\end{array}$ & $\begin{array}{l}\text { que }<* \text { QUI/ } \\
\text { QUIA 'le fait } \\
\text { que' }\end{array}$ & & & & & \\
\hline $\begin{array}{l}\text { Conj. de } \\
\text { coordination } \\
\text { négative }\end{array}$ & $n i \ldots n i<\mathrm{NEC}$ & & & & & \\
\hline $\begin{array}{l}\text { Termes de } \\
\text { polarité } \\
\text { négative }\end{array}$ & $\begin{array}{l}\text { quiconque } \\
<\text { QUICUMQUE }\end{array}$ & $\begin{array}{l}\text { un/une } N \\
\text { quelconque } \\
\text { <QUICUMQUE }\end{array}$ & \begin{tabular}{|l|} 
le/la \\
moindre $N$ \\
que ce soit
\end{tabular} & \begin{tabular}{|l|} 
qui que \\
ce soit / \\
quoi que \\
ce soit
\end{tabular} & $\begin{array}{l}\text { âme qui } \\
\text { vive }\end{array}$ & \\
\hline $\begin{array}{l}\text { Termes de } \\
\text { libre choix } \\
\text { (hypothétiques/ } \\
\text { interrogatifs) }\end{array}$ & personne & rien & jamais & & & \\
\hline
\end{tabular}

Dans l'analyse de corpus, seuls les termes négatifs de la partie blanche seront considérés. Le seul élément sans valeur proprement négative qui sera retenu est le terme de restriction que, qui se comporte de manière analogue aux pronoms et adverbes négatifs et qui est traditionnellement inclus dans ce type d'analyse (cf. de Ashby 1976, 1981, 2001, Armstrong/Smith 2002, Coveney ${ }^{2} 2002$ et Hansen/Malderez 2004, entre autres). Par contre, les termes de polarité, dans la partie grise du tableau, n'expriment pas la négation de phrase et seront donc exclus de l'analyse.

35 Toutes les étymologies $c f .<$ http://www.larousse.fr/dictionnaires/francais $>$. 


\subsection{La variable $\pm n e$ dans les analyses empiriques}

\subsection{1 \pm ne dans les corpus graphiques}

\subsubsection{1 \pm ne dans les textes $d u 17^{e}$ au $19^{e}$ siècle}

Malgré l'absence de corpus oraux avant le $20^{\mathrm{e}}$ siècle, l'analyse prudente des sources graphiques disponibles fournit quelques pistes concernant l'origine de l'omission du ne de la négation en français. En (40) figure une vue d'ensemble des pourcentages de la variante $+n e$ dans les textes littéraires enregistrés dans la base de données Frantexte ${ }^{36}$.

(40) L'absence de ne dans Frantexte entre 1700 et 1999 selon Dufter (2013)

\begin{tabular}{|lllllll|}
\hline & $1700-1749$ & $1750-1799$ & $1800-1849$ & $1850-1899$ & $1900-1949$ & $1950-1999$ \\
\hline -ne & 1 & 10 & 76 & 246 & $2^{\prime} 422$ & $5^{\prime} 668$ \\
Tot. nég & $7{ }^{\prime} 396$ & $14^{\prime} 598$ & $18^{\prime} 631$ & $20{ }^{\prime} 479$ & $36^{\prime} 579$ & $34^{\prime} 902$ \\
$\%+n e$ & $>99,9 \%$ & $>99,9 \%$ & $99,6 \%$ & $98,2 \%$ & $93,4 \%$ & $83,8 \%$ \\
\hline
\end{tabular}

Les pourcentages de la variante $+n e$ dans les textes littéraires présentés en (40) permettent d'affirmer que l'absence de ne reste jusqu'à nos jours un fait isolé.

Cependant, hors du domaine littéraire, la question de savoir à quel moment l'absence de ne a commencé à se répandre est fortement débattue. Dans les textes théâtraux et les journaux intimes du $17^{\mathrm{e}}$ siècle, la particule ne semble encore catégorique. En ce qui concerne le $18^{\mathrm{e}}$ siècle, Séguin (1972: 255), cité par Martineau/Mougeon (2003: 120), attribue l'absence de ne à «d'obscurs gratte-papiers qui sont parfois presque illettrés». Finalement, Dufter (2013) rapporte que dès le $19^{\mathrm{e}}$ siècle les omissions de ne seraient liées aux contextes d'imitation littéraire du langage parlé.

$\mathrm{Au}$ niveau extralinguistique, les facteurs historiques les plus influents pour \pm ne semblent être la provenance et la nature du corpus 
ainsi que la stratification sociale des locuteurs représentés. Aussi bien au Canada qu'en Europe, le phénomène semble émerger des classes défavorisées et affecter au fur et à mesure les classes supérieures (cf. change from below). D'un point de vue géographique, l'extension de l'omission du ne au tournant du $19^{\mathrm{e}}$ siècle semble plus rapide au Canada qu'en Europe. Tandis que dans un corpus de lettres privées de Québécois du $17^{\mathrm{e}}$ et $18^{\mathrm{e}}$ siècle le ne semble presque catégorique, au $19^{\mathrm{e}}$ siècle, dans un journal intime québécois, l'omission de ne s'élève presque à 40\% (cf. Martineau/Mougeon 2003: 134). Martineau/ Mougeon (2003) suggèrent quel'expansion de l'omission du ne débute au $18^{\mathrm{e}}$ siècle, alors que Martineau (2009: 169) montre que le phénomène était déjà bien répandu au cours du $18^{\mathrm{e}}$ siècle. En effet, dans un autre corpus de lettres de Canadiens francophones, 20,7\% des négations de phrase ne sont exprimées que par pas (cf. Martineau 2009: 169, $c f$. aussi Coveney ${ }^{2} 2002$ : 58-59).

Paradoxalement, Dufter/Stark (2007) trouvent dans le journal d'Héroard ( $c f$. Ernst 1985), qui représente la production transcrite du jeune roi Louis XIII au début du 17 e siècle, des pourcentages importants d'omissions de $n e$ (seulement $27 \%+n e$ avec des sujets clitique et la particule négative pas, à l'âge de 5 à 9 ans, $c f$. Dufter/Stark 2007: 122) ${ }^{37}$. Exactement comme dans les corpus oraux récents (cf. section 2.2.2), les contextes linguistiques favorisant l'omission de ne dans les sources historiques sont les contextes de sujets clitiques (cf. Dufter/Stark 2007, Martineau/Mougeon 2003: 144 et section 2.2.5.1) et les combinaisons

37 Les corpus de Martineau/Mougeon (2003) et de Dufter/Stark (2007) reflètent deux réalités linguistiques différentes du $17^{\mathrm{e}}$ siècle. D'une part, le corpus de Martineau/Mougeon (2003) se compose de lettres de famille, journaux intimes et textes théâtraux. D'autre part, le journal d'Héroard est destiné à une fin presque scientifique: Jean Héroard, le premier médecin du dauphin, note scrupuleusement le développement linguistique, gestuel et social du futur roi et met ainsi à disposition une source extraordinaire pour l'étude du langage enfantin d'un individu de la classe parisienne supérieure. Nous avons tout de même classé ce corpus parmi les corpus graphiques, car Jean Héroard ne disposait pas des moyens techniques modernes pour enregistrer et transcrire la production langagière du dauphin et s'en est remis uniquement à sa perception. 
sujet-verbe-marque-négative fréquentes ( $c f$. Dufter 2013), surtout avec c'est et faut sans explétif ( $c f$. Martineau/Mougeon 2003 : 130).

Pour conclure, les différentes sources graphiques du $17^{\mathrm{e}}$ au $19^{\mathrm{e}}$ siècle montrent que les textes littéraires ainsi que la communication privée restent fidèles à la norme jusqu'à l'époque moderne, tandis que la production langagière phonique, transcrite au $17^{\mathrm{e}}$ siècle par Héroard, manifeste déjà un patron de variation du \pm ne comparable à celui observé dans les corpus phoniques transcrits au $20^{\mathrm{e}}$ siècle ( $c f$. section 2.2.2). Une comparaison prudente des corpus historiques confirme donc la thèse de Dufter/Stark (2007), Martineau (2011) et Poplack/St-Amand (2009) d'une variation \pm ne ancienne et plus ou moins 'stable' depuis le $17^{\mathrm{e}}$ siècle ( $c f$. la discussion en section 2.1.2.2).

\subsubsection{2 \pm ne dans la communication électronique au $20^{e}$ et $21^{e}$ siècle}

Avec la communication médiatisée par ordinateur (angl. computer mediated communication, $\mathrm{CMC}$ ), la constitution de grands corpus graphiques qui reflètent le langage spontané de manière authentique est devenue beaucoup plus facile qu'auparavant.

En ce qui concerne la variation $\pm n e$ dans les textes électroniques modernes, les taux de ne diffèrent selon les mêmes facteurs linguistiques et extralinguistiques que dans le français phonique transcrit au $17^{\mathrm{e}}$ siècle et celui d'aujourd'hui ( $c f$. sections 2.2.1.1 et 2.2.2). Au niveau extralinguistique, la réalisation de ne dépend des conditions du discours, comme les chiffres globaux en tableau (41) le montrent.

(41) $\pm n e$ dans l'écriture électronique

\begin{tabular}{|llll|}
\hline Type de communication & $+n e$ & \\
\hline Synchrone & IRC messenger & $2,78 \%$ & van Compernolle/Williams 2007 \\
& chat & $16 \%$ & van Compernolle 2008b \\
\cline { 2 - 3 } Asynchrone & clavardage modéré & $92,16 \%$ & van Compernolle/Williams 2007 \\
& forums de discussion & $46,3 \%$ & \\
& annonces de contact en ligne & $77,6 \%$ & van Compernolle 2008a \\
& (Québec) & & \\
& SMS (Suisse) & $23,5 \%$ & Stark (2012) \\
\hline
\end{tabular}


La communication synchrone non-surveillée (messenger, chat) manifeste des taux de ne proches de zéro. Par contre, dans le clavardage modéré, c'est-à-dire dans une interview tapée synchroniquement en ligne, ne est réalisé presque catégoriquement. De la même façon, les annonces de contact québécoises (une forme de communication asynchrone) contiennent également un pourcentage de ne assez élevé, fait expliqué par van Campernolle (2008a) à l'aide du concept de l'audience design ( $c f$. Bell 1984, 2001 et section 2.3.4). Finalement, dans les SMS suisses, analysés par Stark (2012), l'emploi de ne n'est que légèrement (plus?) élevé par rapport à la communication synchrone via chat ou à celle phonique ( $c f$. section 2.2.2).

Au niveau linguistique, van Compernolle (2008b) et Stark (2012) prouvent que, comme dans le domaine phonique, le facteur principal est le type de sujet ( $c f$. section 2.2.5.1).

D'une manière générale, l'emploi de $\pm n e$ dans la CMC dépend fortement du type de communication (synchrone vs. asynchrone/ surveillée vs. non-surveillée) et est soumis, comme dans la phonie, à des régularités linguistiques, en particulier au type de sujet ( $c f$. section 2.2.5.1).

\subsection{2 \pm ne en français contemporain: les analyses de corpus phoniques}

Les travaux les plus importants concernant la variable \pm ne ont été effectués sur des corpus électroniques phoniques. Ceux-ci nous permettent de dresser une image détaillée de la variation du ne de négation dans la langue phonique depuis la deuxième moitié du $20^{\mathrm{e}}$ jusqu'au début du $21^{\mathrm{e}}$ siècle.

Dans le diagramme (42) apparaissent, par ordre chronologique selon la constitution des corpus, les taux globaux de + ne dans 20 corpus de communication phonique directe (angl. face-to-face), enregistrés entre 1940 ( $c f$. Poplack/St-Amand 2009) et 2004 (cf. Culbertson 2010). 


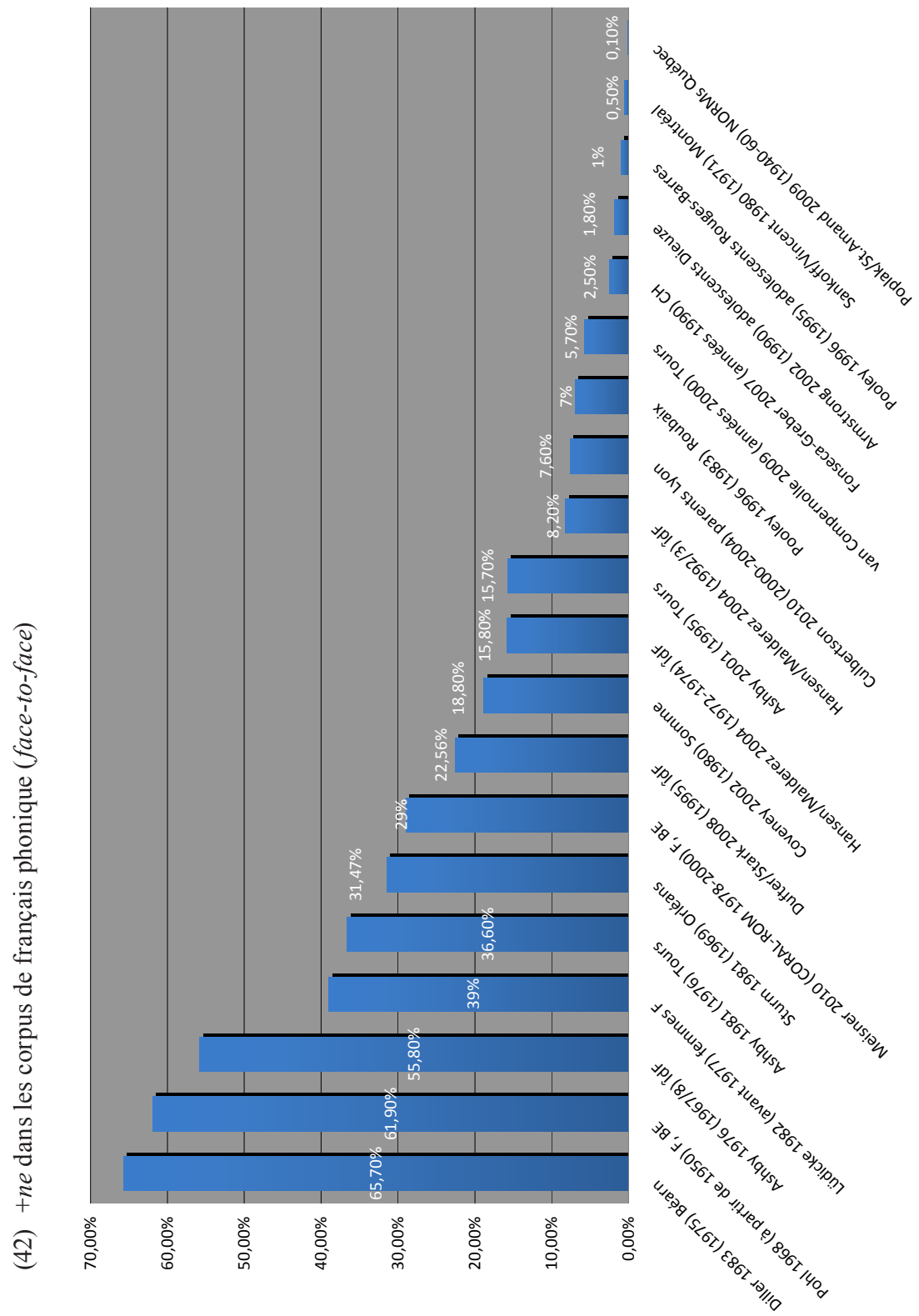


Dans le diagramme (42) figurent d'abord les noms des chercheurs et l'année de publication, puis, entre parenthèses, l'année de constitution du corpus et le lieu de son enregistrement. Si un groupe spécifique de locuteurs a été envisagé dans l'étude, nous le notons avant l'indication du lieu. Les corpus sans spécification contiennent des données de locuteurs et locutrices adultes. Il est à noter que la plupart des corpus cités a été établie à l'aide d' interviews sociolinguistiques.

Les données en (42) montrent que, dans la deuxième moitié du $20^{\mathrm{e}}$ siècle, les taux globaux de $+n e$ en communication phonique directe se situent entre $0,1 \%$ +ne, fait déjà constaté par Poplack/St-Amand (2009) dans les années 1940 chez des locuteurs québécois âgés et issus de milieux, et 65,7\% +ne, mesuré par Diller (1983) en 1975 chez des locuteurs des milieux ruraux du sud-est de la France. Mis à part le corpus de Diller (1983), seuls deux autres corpus montrent davantage de négations bipartites que monopartites: le premier a été enregistré en 1950 par Pohl (1968) en Belgique et en France, et le second à Paris en 1967/8 par Ashby (1976).

Il est important de noter que dans les 17 corpus phoniques restants, enregistrés entre 1940 et 2004, la marque de 50\% +ne n'est plus atteinte. Lüdicke (1982) constate un taux de 39\% +ne dans la production langagière de cinq françaises de classe moyenne. En 1976, Ashby (1981) recueille un corpus à Tours qui ne manifeste que 36,6\% +ne. Sturm (1981), pour sa part, enregistre dans le corpus de l'Étude sociolinguistique sur Orléans un taux de $31,47 \%+n e$. Le corpus $C$-ORAL-ROM, constitué par l'équipe du GARS entre 1987 et 2000 dans différentes régions de France et de Belgique ( $c f$. Cresti/Moneglia 2005) et analysé par Meisner (2010), manifeste un pourcentage de $29 \%+n e$. Quant à Dufter/Stark (2007), ils observent un pourcentage de $22,56 \%$ +ne dans un corpus enregistré en 1995 en Île-de-France, et Coveney (2002) mesure en 1980 un taux de $18,8 \%+$ ne dans la Somme. Même s'il a été constitué relativement tôt, à savoir entre 1972 et 1974, le corpus Péretz-Juillard, analysé par Hansen/ Malderez (2004), manifeste déjà un taux de ne de seulement 15,8\% +ne. En 1995, presque vingt ans après son premier enregistrement à Tours, Ashby (2001) constitue un deuxième corpus tourangeau, comparable au premier, qui ne manifeste que $15,7 \%$ +ne. De la même façon, Hansen/ 
Malderez (2004) enregistrent, environ vingt ans après leur premier corpus, un deuxième corpus en Île-de-France afin de saisir le changement en temps réel. Comme Ashby (2001), elles constatent un net déclin dans un laps de temps de vingt ans: le taux de ne passe de $15,8 \%+n e$ dans les années 1970 à seulement 8,2\% +ne dans les années 1990.

En ce qui concerne le langage adulte dirigé vers l'enfant, Culbertson (2010) constate une taux de 7,6\% +ne dans le Corpus de Lyon, recueilli entre 2000 et 2004. La première des deux études de Pooley (1996) sur un corpus enregistré à Roubaix révèle un taux de $7 \%+n e$. Van Compernolle (2009) trouve dans des conversations détendues enregistrées à Tours un taux de 5,7\% +ne.

Dans les cinq corpus restants, spécifiés géographiquement ou en fonction de l'âge des locuteurs, la réalisation de ne est proche de zéro. Le corpus de Fonseca-Greber (2007), qui manifeste un taux de 2,5\% +ne, a été enregistré dans les années 1990 en Suisse, où la production de ne chez les adultes est presque aussi restreinte que celle constatée chez les adolescents en France par Armstrong (2002) et Pooley (1996): $1,8 \%+n e$ à Dieuze et $1 \%+n e$ à Rouges-Barres.

Finalement, les pourcentages les plus bas de ne sont constatés en français canadien, et ceci déjà depuis les premiers enregistrements: Poplack/St-Amand (2009) observent un taux de 0,1\% + ne dans les Récits du français québécois d'autrefois, enregistrés depuis les années 1940 dans les régions québécoises rurales. Sankoff/Vincent (1980) confirment, dans un corpus enregistré en 1971 à Montréal, qu'au Canada l'usage de ne reste stable à un niveau très bas d'environ $0,5 \%+n e$. Dans le français phonique québécois, le ne de négation est désormais jugé obsolète, même si des données plus récents ne sont pas disponibles (il n'existe que des corpus récents de CMC québécois, $c f$. van Compernolle 2008a, discuté en section 2.2.1.1).

Par conséquent, il est possible d'affirmer que dans la communication directe, les adolescents français et les adultes suisses manifestent dans les années 1990 des taux de ne proches de zéro, qui étaient déjà observables au Québec cinquante ans auparavant.

Néanmoins, il est toujours possible de trouver des taux considérablement élevés de $n e$ en français phonique européen, notamment dans les 
émissions radiophoniques. Dans le diagramme (43) figurent, également classés par ordre chronologique, les taux de $+n e$ constatés dans les corpus radiophoniques entre 1960 et 1997.

(43) +ne dans les corpus de français radiophonique

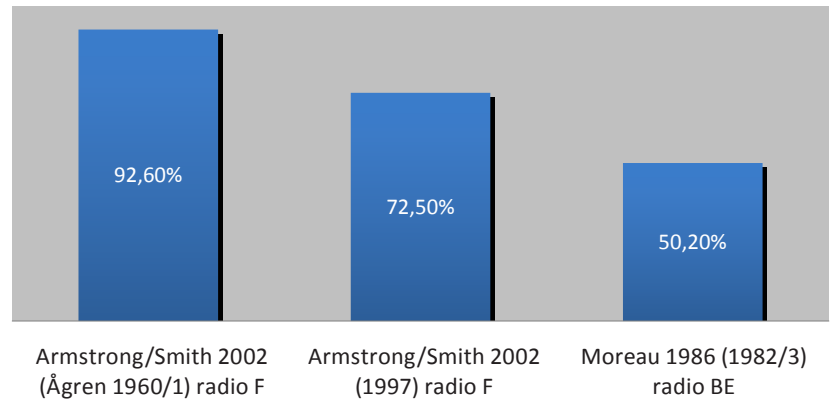

Dans une analyse en temps réel, Armstrong/Smith (2002) comparent un corpus d'émissions culturelles et politiques, enregistré par Ågren (1973) au début des années 1960, à un corpus similaire constitué presque quarante ans après. Ils observent un déclin d'environ 20\%, passant d'un taux très élevé de +ne dans les années $1960(92,6 \%+n e)$ à un chiffre toujours considérable de $72,5 \%+n e$ vers la fin des années 1990 . Le corpus de Moreau (1986), enregistré dans les années 1982/3 en Belgique, se place chronologiquement entre les deux corpus cités précédemment et contient, tout comme les corpus d'Armstrong/Smith (2002), des interviews avec des créateurs culturels très cultivés. La réalisation de $n e$ dans le corpus belge de Moreau (1986) dépasse la moitié des cas (50,2\% $+n e)$ mais est inférieure à celle mesurée dans les deux autres corpus, ce qui pourrait être dû à sa provenance géographique.

Quant à l'évolution récente de la négation en français phonique au tournant du $21^{\mathrm{e}}$ siècle, nous pouvons conclure, tout d'abord, que la marge de variation de ne dans les corpus est énorme. Deuxièmement, nous constatons un net recul de ne, confirmé indépendamment par trois analyses en temps réel (Ashby 2001 pour le français tourangeau, Hansen/ Malderez 2004 pour la région parisienne et Armstrong/Smith 2002 pour le français radiophonique de France). Cependant, il est nécessaire 
de noter que, malgré le déclin empiriquement fondé de ne, cette particule ne semble pas disparaitre complètement du français phonique. Ceci nous permet d'affirmer que la variation du ne dans les corpus de français phonique, que nous n'avons présentée jusqu'ici qu'en termes de taux globaux de $+n e$, mérite une considération plus approfondie, afin de détecter les facteurs (extra)linguistiques qui influencent l'emploi de $n e$.

\subsubsection{Tendances générales: l'influence des facteurs (extra)linguistiques sur \pm ne}

Les tableaux (44) et (45) montrent les influences généralement observées des facteurs intra- et extralinguistiques susceptibles d'influencer la réalisation de ne en français phonique.

(44) Les influences générales des facteurs extralinguistiques sur \pm ne

\begin{tabular}{|c|c|c|}
\hline & $+n e$ & ne \\
\hline $\begin{array}{l}\text { Diachronie: l'année de la constitution du corpus } \\
\text { (cf. Armstrong/Smith 2002, Ashby 2001, Hansen/ } \\
\text { Malderez 2004) }\end{array}$ & années 1950-1970 & après 1980 \\
\hline $\begin{array}{l}\text { Diatopie: le lieu de la constitution du corpus } \\
\text { (cf. Coveney } 22002 \text {, Fonseca-Greber 2007, Poplack/ } \\
\text { St-Amand 2009, Sankoff/Vincent 1980) }\end{array}$ & $\begin{array}{l}\text { le sud de la } \\
\text { France }\end{array}$ & $\begin{array}{l}\text { Canada, } \\
\text { Suisse, le nord } \\
\text { de la France }\end{array}$ \\
\hline $\begin{array}{l}\text { Âge des locuteurs ( } c f \text {. Armstrong 2002, Ashby 1981, } \\
\text { Coveney }{ }^{2} 2002 \text { ) }\end{array}$ & âgé & jeune \\
\hline $\begin{array}{l}\text { Diastratie: classe sociale et formation des locuteurs } \\
\text { (cf. Ashby 1976, 2001, Hansen/Malderez 2004) }\end{array}$ & privilégié, cultivé & $\begin{array}{l}\text { défavorisé, } \\
\text { moins cultivé }\end{array}$ \\
\hline $\begin{array}{l}\text { Situation de communication ( } c f \text {. Armstrong } 2002 \text {, } \\
\text { Armstrong/Smith 2002, Ashby 1981, Coveney } \\
{ }^{2} 2002 \text {, Meisner 2010) }\end{array}$ & $\begin{array}{l}\text { distance } \\
\text { communicative, } \\
\text { radio }\end{array}$ & $\begin{array}{l}\text { immediat } \\
\text { communicatif, } \\
\text { face-to-face }\end{array}$ \\
\hline
\end{tabular}

Il semble que les différents groupes de locuteurs manifestent le même phénomène de variation morphosyntaxique, mais qu'ils se trouvent à des stades différents de son évolution. Le français canadien pourrait être conçu comme le stade le plus avancé, vu que ne y est très rare depuis au moins déjà 70 ans (cf. Poplack/St-Amand 2009, Sankoff/Vincent 1980), suivi en Europe par le français de Suisse (Fonseca-Greber 2007). 
En Belgique et en France, les locuteurs adultes, surtout au sud de la France, manifestent toujours des taux non-négligeables de ne, alors que les jeunes français ( $c f$. Armstrong 2002) montrent des taux de ne comparables aux adultes canadiens ou suisses. En France ( $c f$. Ashby 1976) et éventuellement en Belgique ( $c f$. Moreau 1986), une provenance sociale élevée ou des études secondaires peuvent freiner l'omission de ne, mais ceci ne vaut pas (ou éventuellement plus) pour la Suisse ni pour le Canada ( $c f$. Fonseca-Greber 2007 et Sankoff/Vincent 1980). Au niveau intrapersonnel, plusieurs études suggèrent que l'omission du ne est plus forte dans les situations de l'immédiat communicatif que dans la distance communicative ( $c f$. Armstrong 2002, Ashby 1981, Coveney 22002, Meisner 2010). Si l'on considère le discours médiatique comme langage de distance, ces résultats correspondent au nombre élevé de négations bipartites trouvées dans les émissions radiophoniques.

(45) Les influences générales des facteurs linguistiques sur $\pm n e$

\begin{tabular}{|c|c|c|}
\hline & $+n e$ & $-n e$ \\
\hline $\begin{array}{l}\text { Fréquence } \\
\text { (cf. Moreau 1986: 152) }\end{array}$ & $\begin{array}{l}\text { structures, éléments et } \\
\text { formes rares }\end{array}$ & $\begin{array}{l}\text { éléments et formes } \\
\text { fréquentes, collocations/ } \\
\text { séquences préformées, } \\
\text { p. ex. [ [epa] pour je sais pas }\end{array}$ \\
\hline $\begin{array}{l}\text { Type de sujet grammatical } \\
\text { (cf. entre autres Armstrong/ } \\
\text { Smith 2002: } 31 \text {, Coveney } \\
{ }^{2} 2002: 73 \text {, Culbertson 2010: } \\
\text { 95, Hansen/Malderez 2004: } \\
\text { 21, Dufter/Stark 2007: 122- } \\
\text { 124, Meisner 2010: 1949, } \\
\text { Moreau 1986: 144) }\end{array}$ & $\begin{array}{l}\text { non-clitique/tonique } \\
\text { (nom propre Paul, DP } \\
\text { le gouvernement, pron. } \\
\text { personnel nous, vous, pron. } \\
\text { relatif qui, pron. indéfini } \\
\text { quelqu'un) }\end{array}$ & clitique $(j e, t u, i l(s))$ \\
\hline $\begin{array}{l}\text { Redoublement du sujet } \\
\text { (cf. Culbertson 2010: } 95 \text {, } \\
\text { Hansen/Malderez 2004: } 21 \text { ) }\end{array}$ & $\begin{array}{l}\text { Sujet lexical sans dislocation } \\
\text { (XP ne V NEG) }\end{array}$ & $\begin{array}{l}\text { Redoublement du sujet } \\
\text { lexical ou du pronom } \\
\text { tonique avec reprise clitique } \\
\text { (XP CL V NEG) }\end{array}$ \\
\hline $\begin{array}{l}\text { Terme négatif } \\
\text { (cf. Armstrong/Smith 2002: } \\
\text { 37, Ashby 1981: 682, } \\
\text { Coveney } 22002: 76, \text { Hansen/ } \\
\text { Malderez 2004: 22) }\end{array}$ & que & pas \\
\hline
\end{tabular}




\begin{tabular}{|c|c|c|}
\hline $\begin{array}{l}\text { Temps verbal } \\
\text { (cf. Hansen/Malderez 2004: } \\
\text { 24, Moreau 1986: 146) }\end{array}$ & formes composées & formes simples \\
\hline $\begin{array}{l}\text { Type de construction } \\
\text { syntaxique } \\
\text { (cf. Ashby } 1981: 678)\end{array}$ & $\begin{array}{l}\text { subordonnée (en particulier } \\
\text { la relative, que+subjonctif et } \\
\text { l'infinitivale); impérative }\end{array}$ & principale, déclarative \\
\hline $\begin{array}{l}\text { Adverbe intensificateur } \\
\text { (cf. Ashby 1976: 123) }\end{array}$ & $\begin{array}{l}\text { avec un adverbe } \\
\text { je (ne) veux absolument pas } \\
\text { aller }\end{array}$ & $\begin{array}{l}\text { sans adverbe } \\
\text { je (ne) veux pas aller }\end{array}$ \\
\hline $\begin{array}{l}\text { Environnement } \\
\text { phonologique (cf. Ashby } \\
\text { 1981: 677-678, Moreau } \\
\text { 1986: 155) }\end{array}$ & $\begin{array}{l}\text { position intervocalique et } \\
\text { postpausale, production } \\
\text { lente }\end{array}$ & $\begin{array}{l}\text { débit rapide, l'omission du } \\
\text { schwa dans le clitique sujet } \\
\text { je/ce permet la formation de } \\
\text { syllabes CV si ne est omis: } \\
\text { [3a.siv.pa] au lieu de } \\
\text { [3na.riv.pa] }\end{array}$ \\
\hline
\end{tabular}

Quant aux facteurs linguistiques, leur influence sur ne se manifeste clairement et constamment à travers les diverses analyses synchroniques et diachroniques, comme la vue d'ensemble en (45) le montre.

D’une manière générale, ne est plus facilement omis dans les structures (ou en adjacence aux formes) de haute fréquence. Ceci inclut avant tout les énoncés aux sujets clitiques phonétiquement légers (je, $t u, i l(s), c e, o n)$, qui semblent à leur tour être des inhibiteurs de la réalisation de ne et qui peuvent donner lieu aux 'séquences préformées' sans ne du type [Jepa] pour je sais pas (cf. Moreau 1986). Cette tendance est surtout valable en combinaison avec une forme verbale simple et fréquente, et un terme négatif ayant les mêmes caractéristiques, comme par exemple pas. En effet, les sujets clitiques semblent être le déclencheur le plus fort pour l'omission du ne. Nous argumenterons par la suite que c'est notamment le caractère atone des sujets clitiques qui empêche la réalisation de ne et que leur fréquence élevée multiplie cet effet dans le discours ( $c f$. chapitre 5).

$\mathrm{Au}$ niveau syntaxique, il semble que les principales et les déclaratives sans adverbe favorisent l'omission de ne. D'un point de vue phonético-phonologique, l'omission de ne devient plus fréquente là où elle permet la formation de syllabes ayant une structure 'idéale' du type CV et dans le parler rapide. 
Dans les sections suivantes, nous traiterons l'influence respective de certains facteurs individuels dans les analyses de corpus. Leurs influences sur $\pm n e$ seront par la suite mesurées dans l'analyse de corpus (cf. chapitre 4).

\subsubsection{L'influence des facteurs extralinguistiques en détail}

\subsubsection{La diatopie}

Compte tenu du fait qu'au Canada la réalisation du ne a presque disparu ( $c f$. Auger 1994), nous nous concentrerons dans cette section sur le français européen, où la diatopie peut faire une différence quant à l'expression de la négation. Ainsi, comme le montre la carte en (46), certaines régions du nord de la France semblent plus touchées par la perte de ne que celles du sud.

(46) L'influence de la diatopie sur $\pm n e$ en France

\section{Les taux de + ne en français européen}

1 Roubaix: 7\% (Pooley, 1996)

2 Rouges-Barres (adolescents): 1\% (Pooley, 1996)

3 Somme: $18.8 \%$ (Coveney, 2002)

4 ÎdF : 8.2\% (Hansen/Malderez, 2004)*

5 ÎdF: $15.8 \%$ (Hansen/Malderez, 2004)*

6 ÎdF : 22.56\% (Dufter/Stark, 2008)*

7 ÎdF: 55.8\% (Ashby, 1976)

8 Dieuze (adolescents): 1.8\% (Armstrong, 2002)

9 Orléans: $31.47 \%$ (Sturm, 1981)

10 Tours: $5.7 \%$ (van Compernolle, 2009)

11 Tours: $15.7 \%$ (Ashby, 2001)

12 Tours: $36.6 \%$ (Ashby, 1981)

13 Suisse romande : $2.5 \%$

(Fonseca-Greber, 2007)

14 Lyon (parents) : 7.6\%

(Culbertson, 2010)

15 Béarn: 65.7\% (Diller, 1983)

* ÎdF : Île de France

Carte de base:

Esri, DeLorme, GEBCO, NOAA NGDC and other contributors;

Sources:

UFSP Language and Space

GISLab (UZH), Esri, USGS, NOAA.

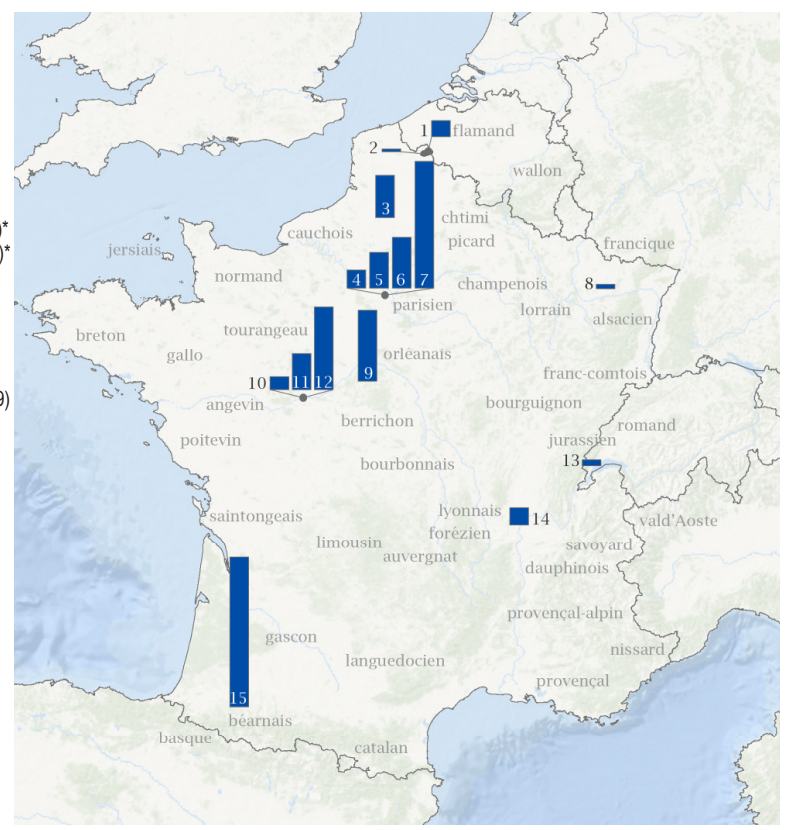


Au centre-nord de l'Hexagone, nous observons non seulement des taux plus restreints de ne, mais également une grande concentration d'analyses de cette variable. Par contre, des données manquent pour d'autres régions comme la Bretagne, la Bourgogne, la Franche-Comté, le Poitou et surtout pour le Midi. La négation monopartite est prévalente au centre-nord et à l'est de la France, plus précisément à Roubaix (proche de la frontière belge), à travers l'Île-de-France jusqu'à Orléans et de Tours jusqu'à Dieuze (non loin de la frontière allemande). En Suisse romande, la négation monopartite est elle aussi utilisée presque sans exception dans tout le territoire. Par contre, en Belgique, Moreau (1986) trouve un taux assez élevé de ne, qui pourrait être dû au fait qu'il s'agit d'analyses de communication radiophonique. Pour la zone d'Oc, au sud de la France, les données sont rares: l'étude de Diller (1983) au Béarn et celle de Culbertson (2010) à Lyon restent relativement isolées. À notre connaissance aucune étude n'a été effectuée dans la zone du Languedoc (en rouge sur la carte). Diller (1983) a enregistré au Béarn dans les années 1970 une très grande présence de ne et Culbertson (2010) observe dans un corpus assez récent un taux de seulement 7,6\%+ne. En absence d'autres analyses récentes dans la zone de la Méditerranée, il est impossible de savoir pourquoi il y a une telle différence entre les résultats de Diller (1983) et ceux de Culbertson $(2010)^{38}$. La seule conclusion claire que nous pouvons tirer de ces résultats est l'existence de disparités nord-sud et est-ouest quant à la réalisation de ne. Cependant, ces résultats devraient être étayés par davantage d'études dans les zones négligées jusqu'à présent, afin de pouvoir ainsi dresser une carte complète de la variation \pm ne en français européen.

Pour des raisons méthodologiques, seuls deux points d'enquête seront représentés dans notre analyse de corpus, à savoir l'Île-de-France et la Suisse romande. L'influence de ces deux zones géographiques sur

38 On pourrait argumenter, tout d'abord, qu'un changement diachronique a eu lieu entre les deux études; deuxièmement, que les différents soustrats dialectaux provoquent cette différence; troisièmement, que c'est le type de corpus qui provoque le décalage entre les résultats des deux analyses (par exemple la conversation parent-enfant dans le corpus de Culbertson 2010). 
la variable \pm ne sera comparée et mise en relation avec d'autres points d'enregistrement ( $c f$. section 4.3.1).

\subsubsection{L'âge des locuteurs}

La coupe transversale à travers les résultats concernant le facteur de l'âge en (47) regroupe, selon leur ordre de parution, les résultats de $\pm n e$ obtenus pour différents groupes d'âge.

(47) L'influence de l'âge des locuteurs sur $\pm n e^{39}$

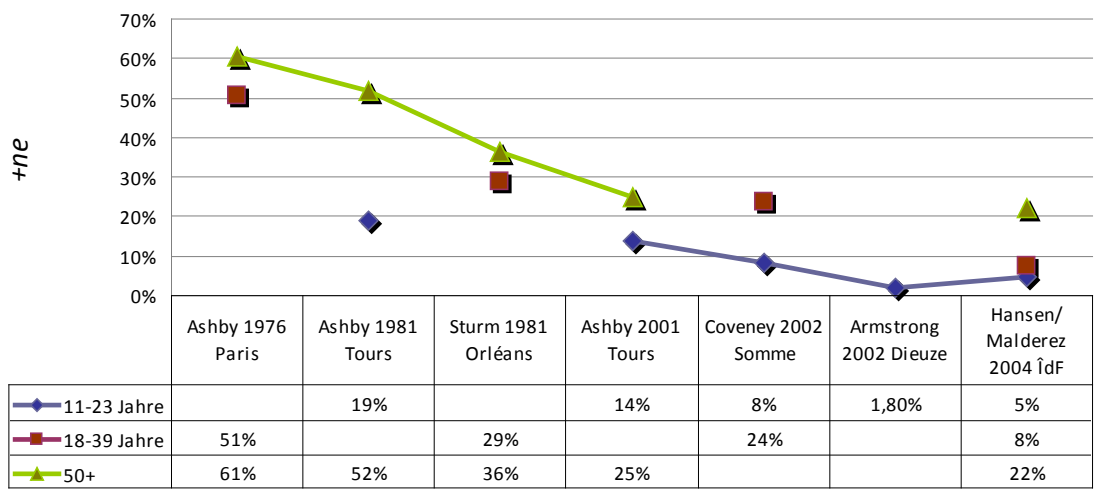

Même si aucune couche d'âge n'est représentée dans toutes les analyses citées en (47), les résultats sont assez clairs: les locuteurs plus âgés (vert), ceux de plus de 50 ans, produisent beaucoup plus souvent des négations bipartites que les locuteurs moyens (rouge) de 18 à 39 ans. Finalement, les jeunes (bleu) de 14 à 23 ans montrent une réalisation constamment basse de ne. Nous pouvons attribuer ces différences à un processus de changement linguistique, comme le soutient Ashby (2001: 21), ou à un effet d'âge (angl. age grading) (cf. Posner ${ }^{2} 2007$, Blanche-Benveniste/Jeanjean 1987).

$39 C f$. Armstrong (2002: 158); Ashby (1976) cité d'après Ashby (2001: 12); Ashby (1981: 682); Ashby (2001: 6), Coveney (22002: 86); Hansen/Malderez (2004: 17); Sturm (1981: 100). Le chevauchement des deux premiers groupes d'âge en diagramme (47) est dû au fait qu'il s'agit d'une synthèse de plusieurs études concernant différentes classes d'âge. 
Le premier processus implique que la production de ne des locuteurs reste plus ou moins stable tout au long de leur vie et que la négation bipartite meurt avec la génération de locuteurs âgés qui l'utilisaient encore. Le second processus implique, par contre, que les locuteurs modifient leur production langagière (phonique) en l'orientant vers la norme graphique tout au long de leur vie. Cependant, il faut noter qu'indépendamment de l'âge des locuteurs, la production de ne subit une régression à travers le temps. Ceci signifie qu'aujourd'hui un locuteur de cinquante ans produit moins de ne qu'un locuteur du même âge il y a trente ans. Nous pouvons donc conclure que les taux de négations bipartites augmentent au cours de la vie d'un locuteur, mais ceci à un moindre degré qu'auparavant ( $c f$. les études longitudinales de Ashby 2001, Armstrong/Smith 2002 et Hansen/Malderez 2004).

Un groupe de locuteurs important, qui ne figure pas dans les études citées en (47), sont les enfants. À ce sujet, Coveney ( ${ }^{2} 2002$ : 65) cite Pohl (1968: 1351-1352), qui trouve que l'absence de ne est une caractéristique saillante du langage enfantin. Labelle (1976) trouve chez les enfants de cinq ans un taux de $5 \%+n e$, tandis que les enfants scolarisés de neuf ans produisent une moyenne de $9 \%+n e$. Lemieux (1985: 95-96) conclut également que, du moins au Québec, la réalisation de ne est dramatiquement liée à la scolarisation et au contact avec la norme graphique. Dufter/Stark (2007) observent un taux de 2 à $8 \%+n e$ dans trois corpus de français enfantin disponibles dans la base de données CHILDES ${ }^{40} \mathrm{Ils}$ remarquent à cet égard que, comme l'absence de ne n'est pas catégorique dans le français enfantin, son usage est probablement lui aussi soumis à des régularités internes ( $c f$. section 2.2.5) qui gouvernent son usage.

Dans l'analyse de corpus, en section 4.3.2, nous comparerons les taux de + ne selon l'âge des locuteurs représentés dans notre corpus. Il s'agit avant tout de locuteurs jeunes, notamment des élèves et des étudiants, mais quelques chercheurs universitaires ainsi que des professeurs y sont aussi représentés. De cette façon, une comparaison limitée de la variation selon l'âge des locuteurs sera possible, même s'il ne s'agira pas d'une analyse exaustive de ce facteur.

$C f .<$ http://childes.psy.cmu.edu/>. 


\subsubsection{La diastratie: la formation et la provenance sociale des locuteurs}

Le diagramme (48) montre les résultats des études précédentes par rapport à l'influence du niveau social et d'instruction des locuteurs.

(48) L'influence de la formation et de la provenance sociale des locuteurs sur $\pm n e^{41}$

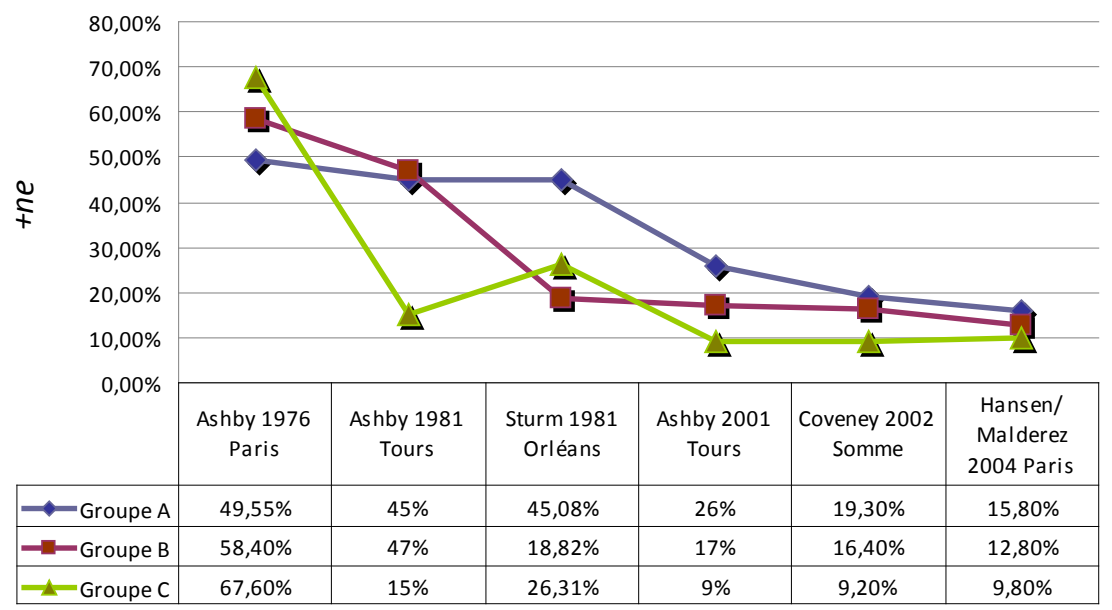

Les structures socio-démographiques des corpus varient et les auteurs cités en (48) appliquent des critères différents ${ }^{42}$, ce qui rend difficile une comparaison directe des résultats. Toutefois, nous pensons que quelques constatations prudentes peuvent être faites.

Dans le diagramme (43), nous distinguons trois niveaux sociaux et d'instruction: une classe supérieure (A), une classe moyenne (B) et une classe inférieure (C). Nous observons une diminution progressive des taux de $+n e$ des trois classes et un inversement de l'ordre des classes

41 Cf. Ashby (1976: 134); Ashby (1981: 683), Ashby (2001: 20); Coveney (2002: 86); Hansen/Malderez (2004: 19); Sturm (1981: 103).

42 Le diagramme (48) montre une synthèse de six études portant sur l'influence de la formation ou de la classe sociale sur $\pm n e$. Les définitions des groupes A, B et $\mathrm{C}$ varient selon la méthodologie appliquée. 
de la première étude par rapport à la plus récente. Dans les premières analyses, nous notons des différences entre les trois classes qui s'évanouissent au fil du temps. Tandis que dans l'étude d'Ashby (1976) la classe $\mathrm{C}$ manifeste les taux les plus élevés, suivie des classes B et A, cet ordre est renversé dans l'étude de Ashby (2001). Les premiers résultats d'Ashby (1976) pourraient alors être interprétés en termes d'une hypercorrection, nivelée progressivement (cf. Dittmar 1997: 32).

Un autre facteur sociolinguistique est à mentionner, à savoir le sexe des locuteurs: dans le corpus d'Ashby (1976), les femmes produisent plus de ne et seraient donc linguistiquement plus conservatrices que les hommes (cf. Ashby 1976: 132), mais dans celui d'Ashby (1981), leurs taux de ne sont plus restreints, ce qui signifierait qu'elles se trouvent dans une phase plus avancée du changement (Ashby 1981: 682). En raison de ces résultats contradictoires, les interprétations de l'influence du sexe restent plutôt vagues.

En ce qui concerne notre analyse, étant donné que dans notre corpus les femmes sont légèrement surreprésentées ( $c f$. section 3.2), le facteur du sexe sera exclu. Dans l'ensemble, et d'un point de vue empirique, le fait que la variante $\pm n e$ soit (encore) un indicateur de la classe sociale en français contemporain reste fort doutable. Toutefois, cette hypothèse, présentée de manière plus détaillée en section 2.3.1, est toujours assez répandue dans la littérature sociolinguistique. Elle sera testée dans notre corpus, dans la limite des données disponibles, en section 4.3.3, puis selon les résultats obtenus ( $c f$. section 5.1.1).

Afin de déterminer les différences concernant la variable \pm ne entre les différents locuteurs dans notre corpus, nous adaptons une approche pilotée par les données ( $c f$. angl. data-driven approach): au lieu de partir des caractéristiques sociolinguistiques des locuteurs, nous partirons de leur production respective de $\pm n e$. Ceci nous permettra de déterminer trois différents types de locuteurs et d'examiner, par la suite, leurs caractéristiques sociodémographiques ( $c f$. section 4.2). 


\subsubsection{La variation situationnelle}

Il existe différentes conceptions de la variation intrapersonnelle, désignée aussi comme variation stylistique, situationnelle, diaphasique ou entre l'immédiat et la distance communicative. En sociolinguistique, les 'styles' ou 'registres' ( $c f$. Coupland 2007, Eckert/Rickford 2001 et Gadet 2004 pour une vue d'ensemble) pourraient être conçus comme des produits de la variation sociale ou même comme son origine (cf. Armstrong 2001, Finegan/Biber 2001). L'on pourrait également les décrire comme une ressource communicative à s'adapter à un interlocuteur ( $c f$. Bell 1984, 2001 et section 2.3.4) ou tout simplement comme l'attention prêtée à la production langagière ( $c f$. Labov 2001). Dans la linguistique variationnelle, par contre, la variation intrapersonnelle est conçue comme faisant partie d'une langue historique ( $c f$. Flydal 1951, Coseriu 1988a,b), c'està-dire qu'elle forme des variétés aux niveaux diatopique, diastratique, diaphasique et éventuellement aussi entre les pôles de l'immédiat et de la distance communicative ( $c f$. Koch/Oesterreicher ${ }^{2} 2011$ et section 2.3.3).

$\mathrm{Au}$ niveau méthodologique, aucune méthode standardisée pour mesurer les différences entre les situations de communication n'existe. Avant l'étude de Meisner (2010), qui examine pour la première fois de façon systématique un corpus représentatif selon la situation de communication, les analyses de ce facteur méthodologiquement exigeant restent largement marginales ou anecdotiques. Ashby (1976) distingue les situations formelles de celles informelles en coupant les interviews en deux parties: la deuxième moitié est conçue comme partie informelle, car les locuteurs seraient plus habitués à la situation et donc plus à l'aise qu'auparavant (cf. Ashby 1976: 131). Dans un travail plus récent, Ashby (1981) enregistre trois locuteurs dans leurs environnements professionnels et privés ( $c f$. Ashby 1981: 681-682). Par la suite, Coveney (2002) observe que l'un de ses informateurs réalise presque le double de ne au bureau que dans une situation de loisirs (en plein air, en présence du chercheur et des enfants). Pour sa part, Meisner (2010) compare systématiquement deux sous-corpus tirés d'un corpus de référence pour le français phonique européen $C-O R A L-R O M$ ( $c f$. Cresti/Moneglia 2005), en se basant pour cela sur les critères communicatifs de l'immédiat et de la distance selon Koch/Oesterreicher (2011). Finalement, 
Pooley (1996) et Armstrong (2002) font une distinction entre les interviews sociolinguistiques, enregistrés par les chercheurs, et les conversations entre peers, desquelles Armstrong (2002) s'absente, tandis que Pooley (1996) reste présent.

Malgré les différences méthodologiques décrites, nous présentons dans le diagramme (49) une comparaison des taux de ne selon différentes situations de communication. La ligne bleue désigne les situations de distance communicative, tandis que la ligne rouge représente les situations de l'immédiat communicatif.

(49) L'influence de la situation de communication $\operatorname{sur} \pm n e^{43}$

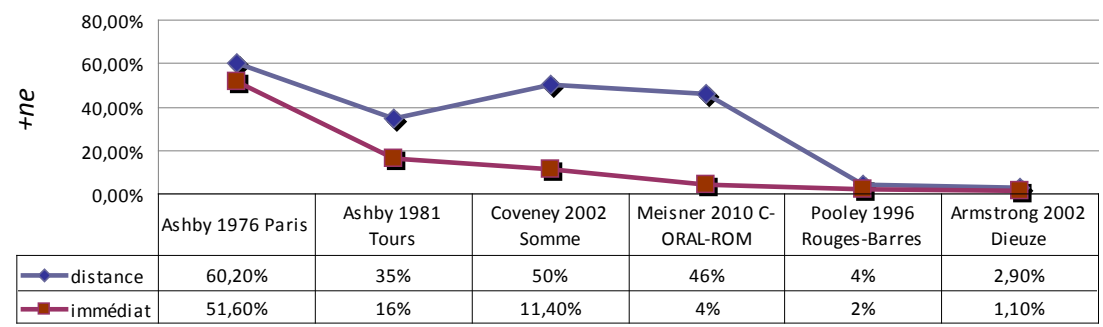

L'écart entre les deux lignes du diagramme (49) est considérable dans certains corpus. Tandis que la ligne bleue, indiquant les situations de distance, zigzague en fonction des corpus, la ligne rouge semble baisser à travers le temps et ne se stabilise qu'à un niveau bas. Le fait que ce soient justement les deux corpus d'adolescents (ceux de Pooley 1996 et d'Armstrong 2002) qui ne montrent presque aucune différence entre les situations de distance et de l'immédiat ne passe pas inaperçu.

Nous proposons, dans ce qui suit, quatre explications possibles de ce fait. Premièrement, le changement linguistique ( $c f$. Ashby 2001 : 21): il est possible que les jeunes aient largement généralisé la négation monopartite indépendamment de la situation de communication (cf. section 2.2.4.2). Deuxièmement, l'effet de l'âge ( $c f$. Posner ${ }^{2} 2007$, Blanche-Benveniste/Jeanjean 1987): l'on pourrait soupçonner que le

43 Cf. Ashby (1976) cité d'après Ashby (2001: 20), Ashby (1981: 681), Armstrong (2002: 158), Coveney (2002: 88), Meisner (2010: 1952), Pooley (1996: 286, valeurs estimées en fonction du diagramme). 
maniement des stratégies communicatives, y compris l'emploi de ne dans les situations de distance communicative, est appris seulement après l'adolescence. Troisièmement, l'on pourrait soutenir que, dû à un changement socio-culturel, les jeunes se servent même dans la distance communicative d'un registre moins formel, qui est réservé chez les locuteurs adultes aux situations de l'immédiat communicatif. Finalement, la méthodologie appliquée par Pooley (1996) et Armstrong (2002) constitue une dernière explication pour les différences minimes entre l'immédiat et la distance chez les jeunes: l'écart entre les interviews et les conversations des peers n'est probablement pas suffisant pour créer des différences notables dans l'expression de la négation.

Dans l'ensemble, chez les adultes, les différences entre les situations de communication peuvent être considérables, mais les méthodes d'enquête pour ce facteur restent perfectibles.

Notre analyse de corpus présente un concept innovateur dans le contexte de la situation de communication: en section 4.3.5, les données authentiques, provenant de situations d'examens oraux et représentant le domaine de la distance communicative, seront comparées aux données provenant de conversations et discussions privées ou menées pendant des cours de langue ( $c f$. section 3.3 pour une description des situations d'enregistrement). De plus, une partie des locuteurs sera présente dans les deux types de situation. Cette approche permettra ainsi de gagner de nouvelles connaissances dans le domaine de la variation intrapersonnelle de \pm ne et de contribuer à la discussion concernant la question théorique de savoir si les locuteurs français vivent réellement dans un état de diglossie, c'est-à-dire dans une alternance continuelle entre une grammaire conçue comme variété 'basse' et une autre conçue comme variété 'élevée'. Cette thèse de la diglossie française (cf. Culbertson 2010, Massot 2010, Zribi-Hertz 2011) sera présentée de manière plus détaillée dans la section 2.3.3 et évaluée en tenant compte des résultats obtenus dans notre corpus (dans la section 5.1.3). De plus, nous discuterons en section 5.3.2 si l'influence de la situation de communication sur la variable \pm ne est réellement autonome, ou bien s'il s'agit plutôt d'un épiphénomène de la distribution des différents types de sujet (comme le soupçonnent Dufter/Stark 2007: 120). 


\subsubsection{L'influence des facteurs linguistiques en détail}

\subsubsection{Le type de sujet grammatical: un continuum quant à la variable \pm ne}

Le type de sujet grammatical est le facteur linguistique le plus important pour la réalisation de ne et s'est avéré pertinent dans toutes les analyses précédentes ( $c f$. entre autres Armstrong/Smith 2002, Ashby 1976, 1981, 2001, Coveney 22002, Hansen/Malderez 2004). Dans ces études, on distingue traditionnellement la classe ouverte des sujets lexicaux de la classe fermée des sujets pronominaux. Les sujets lexicaux sont ici traités en bloc et dans le cas des sujets pronominaux, les taux de $\pm n e$ respectifs de chaque pronom sont établis. En règle générale, les phrases négatives contenant un sujet ouvert sont opposées aux impératives et aux constructions infinitives. Les sujets doublés ou disloqués ne sont que très rarement analysés en tant que troisième classe de sujets (seuls Culbertson 2010 et Hansen/Malderez 2004 les incluent comme tels).

Dans le diagramme (50) figure une vue d'ensemble des types de sujets qui favorisent habituellement la réalisation de $n e$.

(50) Les types de sujet qui favorisent la réalisation de $n e^{44}$

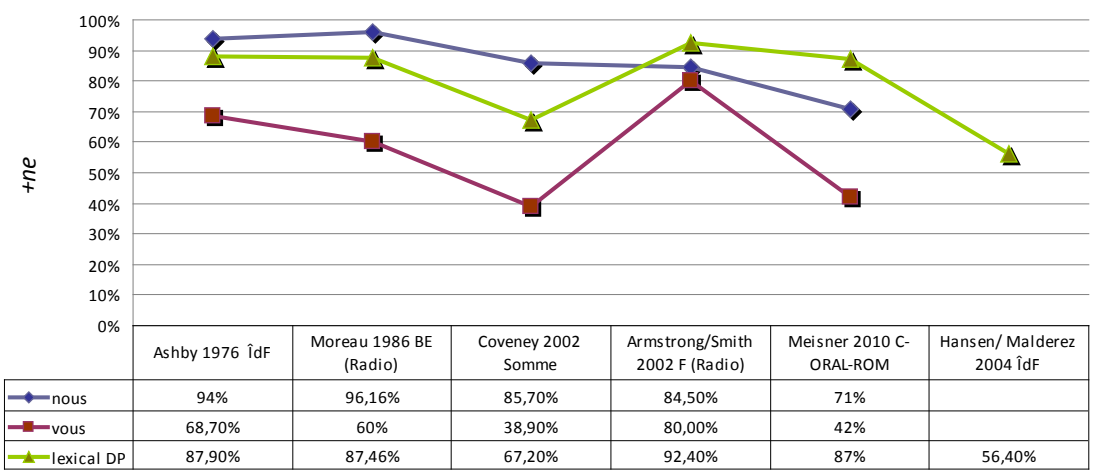

44 En ce qui concerne les données dans le diagramme (50) cf. Ashby (1976: 124); Coveney (2002: 73); Moreau (1986: 144); Hansen/Malderez (2004: 21); Armstrong/Smith (2002: 31); Meisner (2010: 1940). 
Ce sont les sujets lexicaux (y compris les pronoms indéfinis comme quelqu'un) et deux pronoms personnels, à savoir nous et vous, qui sont le plus souvent réalisés avec ne (les taux de nous sont d'ailleurs les plus élevés dans trois des corpus). Les taux de nous et des sujets lexicaux restent stables au-dessus de la marque de 50\% +ne. Les taux pour les sujets lexicaux se situent entre $92,4 \%+n e$, dans le corpus radiophonique d'Armstrong/Smith (2002), et 56,4\% +ne, dans le corpus d'Hansen/ Malderez (2004). Les taux de vous se situent clairement au-dessous des deux autres types de sujet, mais restent, à l'exception des corpus de Coveney (2002) et de Meisner (2010), également largement au-dessus de la marque de $50 \%+n e$.

Nous constatons que c'est surtout la ligne représentant vous qui ondule en fonction des différents corpus. Si la moyenne du corpus est particulièrement élevée, comme celle du corpus radiophonique d'Armstrong/Smith (2002), ou restreinte, comme celle d'Hansen/Malderez (2004), les taux de vous le sont également. Par contre, la représentant nous et à un moindre degré également celle des sujets lexicaux semblent à peu près stables.

La fréquence variable des éléments en question constitue un point problématique de ces analyses. Dans les cas où la base empirique des taux de \pm ne consiste seulement en une ou deux occurrences, nous avons éliminé les taux respectifs dans le diagramme (50) et dans les diagrammes suivants (cf. nous et vous dans l'étude d'Hansen/Malderez 2004). C'est donc pour cette raison que les pronoms indéfinis négatifs personne et rien ainsi que les déterminants négatifs aucun/nul $\mathrm{N}$ ne figurent pas dans le diagramme (50). Ceux-ci semblent favoriser la réalisation de ne, mais leurs fréquences en position de sujet dans les corpus sont souvent trop restreintes pour pouvoir tirer des conclusions statistiquement valides.

Par contre, il y a un groupe de pronoms de haute fréquence qui manifeste des taux de ne toujours inférieurs à $50 \%+n e$, à savoir les clitiques je, $c e$ et $t u$. Leurs taux de $+n e$ sont nettement plus bas que les taux des autres sujets et surtout dans le cas de $c e$, l'absence de ne semble presque fixée ${ }^{45}$

45 Sturm (1981: 137) note: «Angesichts des hohen Ausfalls von NE ist es hier ohne Zweifel berechtigt, c'estpas als weitgehend erstarrte, feste Form zu bezeichnen, in der ein NE fast als Fremdkörper bezeichnet werden muß». 
dans certaines séquences. Dans les corpus de Coveney (2002), Hansen/ Malderez (2004) et Meisner (2010) ce manifeste une fréquence de +ne proche à zéro.

(51) Les types de sujet qui favorisent l'omission de ne

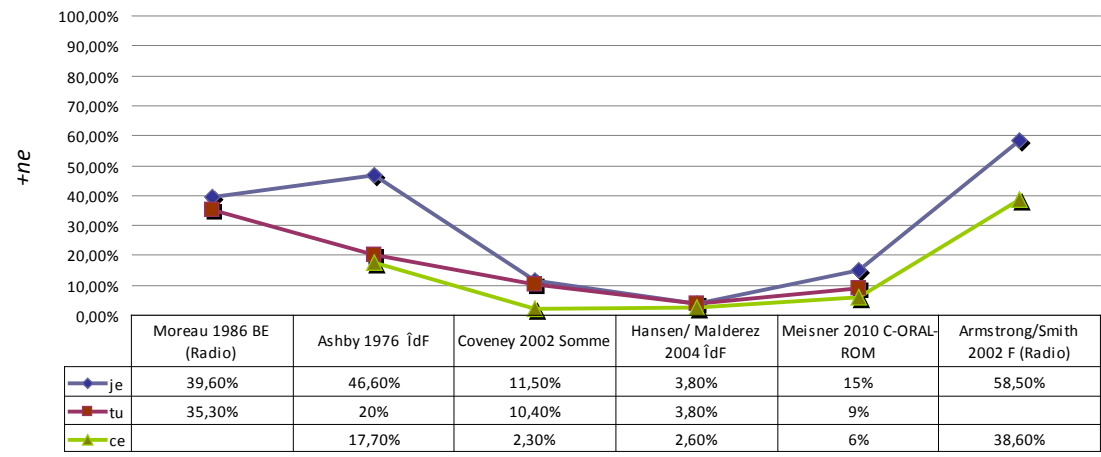

Finalement, il reste un groupe de pronoms dont l'influence sur \pm ne est moins évidente, du moins selon les analyses de corpus disponibles. Il s'agit d'un ensemble hétérogène composé des pronoms personnels il(s), elle(s), du pronom impersonnel ça et du relatif qui. Les taux de ne de ce groupe figurent dans le diagramme (52).

(52) Les types de sujet sans influence claire sur $\pm n e$

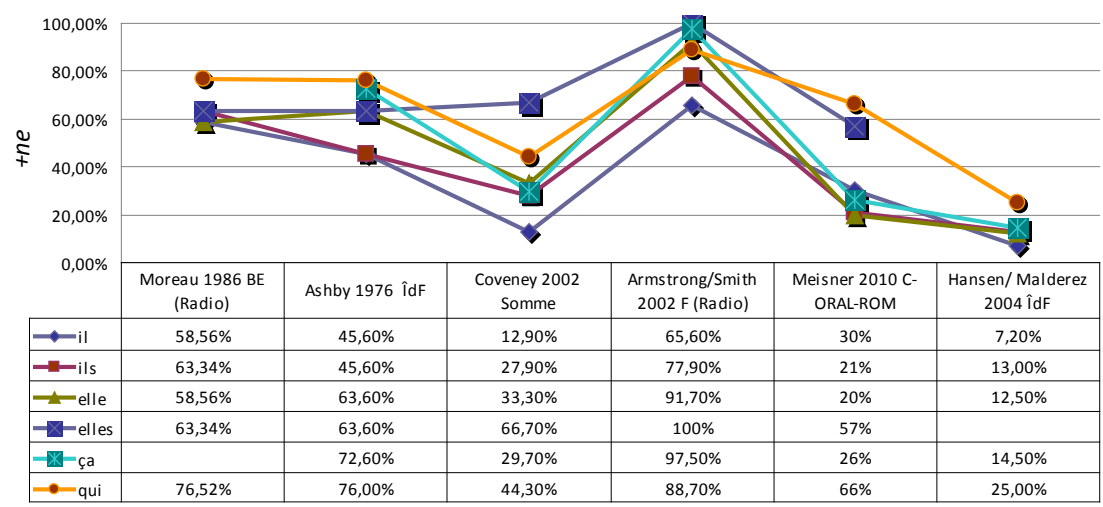


Les lignes du diagramme (52) forment une bande assez compacte, qui monte et descend en fonction des différents corpus. Certaines lignes manifestent un parallélisme frappant: par exemple, les taux de +ne pour qui se situent toujours au-dessus de ceux pour il. Dans l'ensemble, il est cependant difficile d'établir une tendance claire et nette pour ce groupe de pronoms.

En résumant les résultats obtenus, ce sont surtout les pronoms nous et vous ainsi que les sujets lexicaux qui incitent à la réalisation de $n e$. Nous rappelons que, dès les années 1980, aucun corpus de communication phonique directe ne manifeste plus de $50 \%$ de négations bipartites dans ces contextes-là. La tendance à la chuteglobale de $+n e$ montrée dans la section 2.2.3 ne se manifeste donc pas avec nous et vous ni avec les syntagmes nominaux (DPs) lexicaux. Au contraire, les sujets pronominaux inhibent la réalisation de ne à différent degrés: l'absence de ne semble déjà presque inévitable avec le clitique démonstratif ce et est en voie de le devenir avec les clitiques je et $t u$. Par contre, les pronoms personnels $i l(s)$ et elle(s) et l'impersonnel ça semblent, tout comme le pronom relatif qui, avoir une influence intermédiaire ou peu claire.

Dans l'ensemble, nous n'observons donc aucune rupture claire et nette quant à $\pm n e$ entre les sujets pronominaux et lexicaux, mais plutôt un continuum entre les sujets phonologiquement légers et lourds:

(53) Continuum des sujets légers et lourds quant à leur influence sur $\pm n e$

\begin{tabular}{|c|c|c|c|c|}
\hline \multicolumn{3}{|c|}{ Type de sujet } & \multicolumn{2}{|l|}{ Exemple du corpus } \\
\hline Lourd & lexical & DP/ nom propre & $\begin{array}{l}\text { DP[le mec qui t'a vu }] \\
\text { DP [le glossaire de Reichenau }] \\
\text { DP }[\text { la dame }] \\
\text { NomPr }[\text { Ulysse }]\end{array}$ & $+n e$ \\
\hline & pronom & indéfini & personne & \\
\hline & & relatif & $q u i$ & \\
\hline & & démonstratif & $c ̧ a$ & \\
\hline & & clitique lourd & nous, vous, elle & \\
\hline Léger & & clitique léger & $j e, t u, i l, c e$ & $-n e$ \\
\hline
\end{tabular}


Dans la partie supérieure du tableau (53) se situent les sujets lourds, qui favorisent la réalisation de $n e$, comme les DPs, les noms propres, et les pronoms non-clitiques ou lourds. Plus l'on descend vers le bas de l'échelle, plus ne disparaît des corpus, jusqu'à être presque introuvable avec les clitiques légers. La classification simple qui oppose les sujets lexicaux aux pronoms ne reflète donc pas la distribution de \pm ne observée dans les corpus, car celle-ci forme plutôt un continuum.

Une autre catégorie de sujets s'ajoute au continuum des sujets simples, à savoir les sujets redoublés (généralement décrits comme dislocations, $c f$. section 2.3.3 pour une discussion). Par sujet redoublé nous entendons des cas comme celui illustré en (54) (cf. Stark 1997 pour un tour d'horizon). Ce type de sujet comprend un pronom tonique, $c f$. (54) a, ou un nom propre, voire un DP lexical, $c f$. (54)b, qui apparait avec un pronom sujet coréférentiel. L'élément non-clitique peut apparaître en position préverbale (cette construction est souvent désignée comme 'dislocation à gauche'), comme en (54)a,b, ou postverbale, $c f$. (54)c, aussi connue sous le nom de 'dislocation à droite'.

(54) Les sujets redoublés

a. $\quad \boldsymbol{m o i}_{1 \mathrm{SG}} \boldsymbol{j e}_{1 \mathrm{sG}}$ l'ai pas cette feuille-ci (0926)

b. Ia métaphore ${ }_{3 \mathrm{SG}} c_{3}$ 'est pas une arme (0676)

c. $\quad c_{3}$ 'est pas mal les autrichiens ${ }_{3 \mathrm{PL}}(0756)$

Le redoublement du sujet, un facteur rarement testé dans les analyses précédentes, semble fortement défavoriser la réalisation du clitique négatif: Culbertson (2010: 95) trouve un taux de 6,7\% + ne, et Hansen/ Malderez (2004: 21) constatent un taux de 9,5\% +ne avec les cas de redoublement contenant un sujet lexical et 3,3\% +ne si le redoublement contient un pronom tonique.

En plus de cela, nous tenons à signaler que la présence de ne est relativement élevée avec les divers types de construction classés dans la catégorie 'sans sujet ouvert' ( $c f$. entre autres Ashby 1976: 123 79\% +ne, Ashby 1981: 679 68\% +ne, Meisner 2010: 1949 80\% +ne), notamment avec les gérondifs, les infinitifs, les participes présents et parfois aussi les impératifs. Dans notre analyse de corpus, nous n'examinerons que les constructions tensées et n'aurons donc aucun résultat concernant 
les gérondifs, les infinitifs et les participes présent. Néanmoins, nous discuterons l'applicabilité de nos résultats à ces types de construction en. section 5.2.4.6.

Pour notre analyse de l'influence du type de sujet sur $\pm n e$ ( $c f$. section 4.4.1), nous nous attendons donc à ce que les taux de ne augmentent proportionnellement à la complexité morphophonologique du sujet, c'est-à-dire que les sujets lourds devraient provoquer des taux de $+n e$ élevés et les sujets légers des taux de $+n e$ très restreints. En ce qui concerne les sujets redoublés, nous attendons également des taux très bas de $+n e$.

Dans la littérature théorique, l'immense influence du type de sujet sur la variable $\pm n e$ n'a jamais été expliquée en détail. Néanmoins, il existe deux auteurs qui effleurent ce sujet. D'une part, Posner (1985: 189) et Larrivée (2014) soutiennent que la présence des proclitiques en général (et non pas seulement des clitiques sujet) serait difficile à prononcer et donc défavorable à la réalisation de ne ( $c f$. section 2.3.2). D'autre part, Culbertson (2010) soutient que dans le français colloquial les proclitiques (à l'exception de ne) seraient désormais devenus des préfixes du verbe et excluraient, par conséquent, l'intervention du clitique de négation ( $c f$. section 2.3.3). Ces deux approches seront évaluées, à la lumière des données de notre corpus, dans les sections 5.1.2 et 5.1.3.

Dans ce travail, nous proposons une explication prosodique de l'influence du type de sujet ( $c f$. section 5.2): à notre avis, les types de sujet favorisent ou défavorisent la présence de la variable ne en fonction de leurs caractéristiques morphophonologiques. Pour comprendre cette affirmation, il est important de concevoir les différents types de sujet en tant que continuum morphosyntaxique et phonologique et non pas comme une dichotomie entre les sujets lexicaux et pronominaux. Pour cette raison, dans la section suivante, la perspective des types de sujets en tant que continum sera épaulée par l'évidence des données linguistiques. 


\subsubsection{Discussion du type de sujet grammatical: un continuum morphosyntaxique et phonologique}

Nous avons vu que, par rapport à la variable $\pm n e$, les sujets pronominaux et lexicaux ne forment pas deux classes clairement délimitées. Ceci vaut également pour le niveau morphosyntaxique et phonologique, comme nous allons le montrer dans ce qui suit. La conception des différents types de sujet en tant que continuum sera cruciale pour l'analyse et l'interprétation de leur influence sur la variable \pm ne dans les chapitres 4 et 5 .

Inspirés par la typologie des clitiques romans selon Bossong (1998) (cf. aussi Cardinaletti/Starke 1999 et Déchaine/Wiltschko 2002 pour des typologies plus formelles), nous avons développé une sous-classification morphophonologique plus détaillée des types de sujet des phrases (négatives) en français:

(55) Continuum syntactico-phonologique entre les sujets légers et lourds

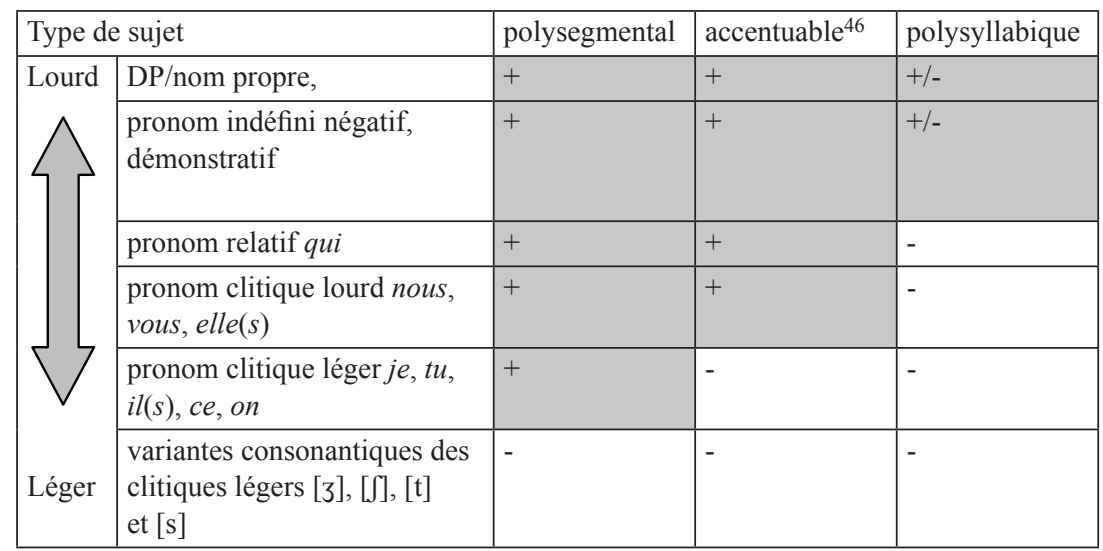

Les sujets lexicaux et les pronoms indéfinis (personne, rien, quelqu'un etc.) sont morphologiquement complexes et syntaxiquement libres, c'est-à-dire qu'ils peuvent être disloqués, séparés du verbe fléchi par

46 Nous considérons comme accentuables les éléments qui peuvent être isolés ou détachés du verbe fléchi et déplacés dans une position accentué. 
une apposition et transférés dans une position accentuée. Le pronom relatif qui, par contre, est monosyllabique mais également séparable du verbe fléchi et accentuable:

(56) Y'en a qui, contrairement à nous, n'ont aucun contact avec leurs voisins. ${ }^{47}$

Paradoxalement, ceci vaut également pour le groupe de pronoms personnels monosyllabiques, que nous appellerons les clitiques lourds, à savoir nous, vous et elle(s). Par contre, dans le même paradigme pronominal (du moins selon la description des grammaires courantes, cf. Riegel/Pellat/Rioul 52008: 199), il y a un groupe très récurrent de formes monosyllabiques et bisegmentales qui se comportent comme des clitiques prototypiques d'un point de vue syntactico-phonologique (cf. Anderson 2005, Kaiser 1992, Zwicky 1977 et Zwicky/Pullum 1983 pour des approches des clitiques dans les langues du monde, ainsi que Heap 2000, Miller/Monachesi 2003, Morin 1979 et Wanner 1987 pour des descriptions des clitiques romans et français), c'est-à-dire, qu'ils ne sont ni séparables de leurs bases ni accentuables. Ce groupe, désigné comme clitiques légers, contient les éléments je, $t u, i l(s), c e$ et on, qui manifestent une allomorphie riche et apparaissent souvent sous forme de variantes monosegmentales consonantiques.

Les clitiques lourds nous, vous et elle(s) sont donc isolables et séparables du verbe fléchi et peuvent être transférés dans une position accentuée, ce qui est impossible pour les clitiques légers je, $t u$, il(s), on et ce, comme les exemples en (57) à (59) le montrent (cf. Kaiser 1992, Kayne 1975, Zwicky 1977 et Zwicky/Pullum 1983 pour les tests de cliticité).

(57) Occurrence des DP et des pronoms dans l'isolement

a. Question: Qui est venu?

Réponse: la femme / Paul / personne / nous / vous / elle(s).

*Je/tu / ill(s) / on.

b. Question: Qu'est-ce qui c'est passé?

Réponse: Ceci / cela / ça.

${ }^{*} \mathrm{Ce}$.

$47<$ http://alwaysquotes.skyrock.com/3106116807-C-est-drole-Y-en-a-qui-contrairement-a-nous-n-ont-aucun-contact-avec.html>, consulté le 15 août 2012. 
(58) Les clitiques légers sont inséparables du verbe

a. *Je, souvent, regarde la télé.

b. *On, par hasard, a trouvé le local.

c. $\quad *$ Ce, heureusement, est ma maison.

(59) Les clitiques lourds sont séparables du verbe

a. Elle(s), souvent, arrive(nt) en retard.

b. Nous, toujours, mangeons ensemble.

c. Vous, malheureusement, n'êtes pas invités.

(60) Contextes particuliers qui permettent l'accentuation ou la séparation des clitiques légers du verbe

a. Je le lui ai dit. (séparation par d'autres clitiques)

b. Je, soussigné(e)........... déclare que... (formule juridique)

c. A: Il t'a appelé?

B: Qui ça, IL? (contexte métalinguistique)

d. Donne-moi-LE! (clitique en position postverbale dans une impérative)

La séparation d'un clitique léger du verbe n'est possible qu'à travers d'autres clitiques, dans des formules juridiques fixes ou dans des contextes métalinguistiques, comme les exemples en (60) le prouvent. L'accentuation d'un clitique n'est possible que s'il est placé en position postverbale, par exemple dans une impérative comme en (60)d. Dans cet exemple, le clitique apparaît en position finale d'un groupe accentuel qui est obligatoirement accentuée en français ( $c f$. section 5.2.2). Malgré les exceptions mentionnées en (60), les clitiques légers préverbaux sont généralement inaccentuables, car la séparation du verbe par d'autres clitiques, $c f$. (60)a, ne permet aucun transfert du clitique dans une position accentuable, et les cas exceptionnels en $(60) \mathrm{b}$ et $\mathrm{c}$ sont très restreints et ne jouent aucun rôle dans notre corpus. Par conséquent, nous retenons que, dans la langue parlée spontanée, les clitiques légers ne sont séparables du verbe que par d'autres clitiques et qu'ils demeurent donc complètement inaccentuables.

Les pronoms sujets dits 'clitiques' ne forment donc aucun ensemble homogène ni en ce qui concerne la réalisation de ne ni au niveau morphosyntaxique et manifestent plutôt des différences syntacticophonologiques considérables, résumées dans le tableau (61). 
(61) Les pronoms sujets clitiques et toniques du français moderne

\begin{tabular}{|c|c|c|c|c|c|}
\hline & Clitique léger & Clitique lourd & Pronom tonique \\
\hline \multirow[t]{4}{*}{$\mathrm{Sg}$} & \multicolumn{2}{|c|}{1} & je & & moi \\
\hline & \multicolumn{2}{|c|}{2} & tu & & toi \\
\hline & \multirow[t]{2}{*}{3} & $\mathrm{~m}$ & il & & lui \\
\hline & & $f$ & & elle & \\
\hline \multicolumn{3}{|c|}{3 sg impersonnel/1pl } & on & & (soi) \\
\hline \multicolumn{3}{|c|}{ Démonstratif } & ce & & ça \\
\hline \multirow[t]{4}{*}{ Pl } & \multicolumn{2}{|c|}{1} & & nous & \\
\hline & \multicolumn{2}{|c|}{2} & & vous & \\
\hline & & $\mathrm{m}$ & ils & & eux \\
\hline & & $f$ & & elles & \\
\hline
\end{tabular}

Les clitiques lourds elle(s), nous et vous sont généralement considérés comme appartenant aussi bien au paradigme des pronoms clitiques qu'à celui des toniques (ou bien d'être homophones dans les deux paradigmes). Contrairement aux clitiques légers je, $t u$, on et $i l(s)$, ces pronoms peuvent être accentués, séparés du verbe fléchi et employés comme réponses isolées.

De plus, les clitiques légers manifestent une allomorphie assez riche en fonction du contexte phonologique, de la situation de communication et du sujet parlant. Dans le tableau (62) figure une vue d'ensemble des variantes des clitiques que nous avons trouvées dans notre corpus ( $c f$. section 4.4.1.2).

(62) Les variantes des clitiques légers trouvées dans notre corpus

\begin{tabular}{|l|cc|c|c|}
\hline \multicolumn{2}{|c|}{} & clitique léger & clitique lourd \\
\hline \multirow{4}{*}{ sg } & 1 & 3ə, 3,, , zero & \\
\cline { 2 - 5 } & 2 & & ty, t & \\
\cline { 2 - 5 } & 3 & $\mathrm{~m}$ & il, i, zero & \\
\cline { 2 - 5 } & & $\mathrm{f}$ & & $\varepsilon 1$ \\
\hline & & ce & sə, s & \\
\hline
\end{tabular}




\begin{tabular}{|c|c|c|c|c|}
\hline 3sg impersonnel/1pl & & on & 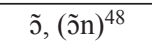 & \\
\hline \multirow[t]{4}{*}{$\mathrm{pl}$} & 1 & & & nu, (nuz) \\
\hline & 2 & & & vu, (vuz) \\
\hline & \multirow[t]{2}{*}{3} & $\mathrm{~m}$ & il, i, (ilz, iz) & \\
\hline & & $\mathrm{f}$ & & $\varepsilon l, \varepsilon,(\varepsilon l z)$ \\
\hline
\end{tabular}

Surtout dans le cas des clitiques légers, nous observons l'existence de formes réduites à une consonne, comme [3] et [ $\mathrm{f}]$ pour je, [t] pour $t u$, [s] pour ce et même des variantes zéro, c'est-à-dire d'absence totale de je et de l'explétif $i l$. Ces variantes réduites, qui ne peuvent pas former une syllabe autonome, sont en partie utilisées dans la langue standard ou dans des contextes pré-vocaliques, comme dans les exemples en (63).

(50) L'emploi 'standard' des variantes consonantiques des clitiques légers

a. j'ai quatorze ans [zekatorzã ] (0634)

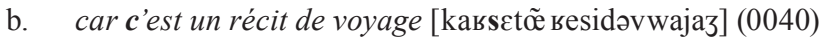

(51) L'emploi 'non-standard' des variantes consonantiques des clitiques légers

а. d'abord je vais vous dire qu'est-ce que l'ironie [dаbовзvevudiskeskəlisoni] (0398)

b. ce que t'as dit [skətadi] (0762)

c. je crois que c'est le quatrième texte [Jkвwakselkatıijemtckst] (0278)

d. y a tout ce qu'il faut [jatuskifo] (0002)

Dans notre corpus, les variantes réduites apparaissent également dans des contextes non-admis par la norme. C'est grâce à la transcription phonétique que nous pouvons identifier ces cas. Nous trouvons, par exemple, [3] et [s] (au lieu de [3ə] et [sə]) pour je et ce devant des consonnes en (64)a et b. Dans l'exemple (64)b figure la variante [t] pour $t u$, ce qui est phonotactiquement logique mais normativement incorrect, et en (64)c, la variante [3] du pronom je apparaît sous forme dévoisée comme [J]. Dans certains cas, les clitiques légers sont élidés, voire complètement fusionnés, comme le il-impersonnel dans l'exemple (64)d.

48 Dans le cas de [õn] il n'est pas clair si [n] fait partie du pronom ou de la particule de négation, par conséquent, ces cas ont été exclus de l'analyse, $c f$. section 3.8. 
Par conséquent, la marge de variation illustrée en (64), allant jusqu'à la réduction à un seul segment consonantique, à la fusion ou à l'élision, est une idiosyncrasie des clitiques légers.

Les pronoms traditionnellement conçus comme 'clitiques' ne sont pas les seuls à se présenter comme un ensemble hétérogène Le groupe des sujets non-clitiques est lui aussi morphosyntaxiquement et sémantiquement tout autre qu'uniforme, comme les exemples tirés de notre corpus en (65) le montrent:

(65) Échantillon de sujets non-clitiques des énoncés négatifs dans le corpus

a. Ulysse $_{\mathrm{DP}(=\text { nom propre })}$

b. l'éloquence $e_{\mathrm{DP}}$

c. le mec qui t'a $v u_{\mathrm{DP}[\mathrm{NP}+\mathrm{Rel}]}$

d. le glossaire de Reichena $u_{\mathrm{DP}[\mathrm{NP}+\mathrm{PP}]}$

e. qui $i_{\text {PronRel }}$

f. $\quad c ̧ a_{\text {PronDém }}$

g. personne $e_{\text {PIN }}$

En (65) figurent les différents types de sujets non-clitiques qui apparaissent dans notre corpus en tant que sujets d'énoncés négatifs. Cet ensemble hétérogène comprend des noms propres, $c f$. (65)a, des syntagmes déterminatifs 'simples', comme en (65)b, ou contenant une phrase relative, $c f$. (65)c, ou encore un syntagme prépositionnel comme dans l'exemple (65)d. Le groupe des sujets non-clitiques contient également le pronom relatif qui, $c f$. (65)e, le démonstratif ça ((65)f) et des indéfinis négatifs, comme illustré en $(65) \mathrm{g}$. Nous tenons à noter d'ores et déjà qu'il existe des différences considérables quant aux taux de $+n e$ à l'intérieur du groupe des sujets non-clitiques. Ce sont surtout les pronoms non-clitiques monosyllabiques comme qui et ça qui manifestent parfois des taux de ne aussi bas que certains clitiques ( $c$. Coveney 22002, Hansen/Malderez 2004, Meisner 2010).

Nous pouvons donc à présent résumer le continuum des types de sujets comme suit: les DPs lexicaux, les noms propres, les pronoms indéfinis négatifs et les démonstratifs apparaissent aussi bien sous forme monosyllabique (par exemple l'homme, Paul, rien, ça) que polysyllabique (par exemple son vou de sagesse, Ulysse, personne, ceci/cela) et comptent donc toujours plus d'un segment. Le pronom relatif qui et 
les clitiques lourds sont monosyllabiques, mais peuvent être séparés du verbe et transférés dans une position accentuée, ce qui n'est pas possible pour les clitiques légers. Ce sont en particulier ces derniers qui manifestent des variantes monosegmentales consonantiques comme $[3],\left[\int\right],[\mathrm{t}]$ et [s] ou qui sont fusionnées voire élidées.

Nous pensons que le continuum décrit ci-dessus est crucial pour la réalisation de $n e$. Son influence sur la variable $\pm n e$ dans notre corpus sera testée dans la section 4.1.1. Dans la discussion en section 5.2, nous proposerons une explication prosodique de cette influence.

\subsubsection{Les proclitiques non-sujets}

D'un point de vue diachronique comparatif, Posner (1985: 189) soutient que non seulement les clitiques sujet légers, mais aussi les proclitiques restants peuvent empêcher la réalisation de $n e$.

(66) L'ordre des proclitiques en français (cf. Jones ${ }^{3} 2007: 252$ )

\begin{tabular}{|lllllll|}
\hline 1 & 2 & 3 & 4 & 5 & 6 & 7 \\
\hline Nominatif & 'négation' & $1^{\text {ère }} / 2^{\text {ème }}$ réfléchi & $3^{\text {ème }}$ accusatif & $3^{\text {ème }}$ datif & locatif & génitif \\
\hline je & ne & me & le & lui & y & en \\
tu & & te & la & leur & & \\
il/elle & & se & les & & & \\
nous & nous & & & & \\
vous & & & & & & \\
ils/elles & & & & & & \\
on & & & & & & \\
\hline
\end{tabular}

Dans le tableau (66), nous présentons l'ordre des proclitiques en français, dont la cooccurrence est déterminée par deux règles: premièrement, seul un élément peut apparaître par colonne, et deuxièmement, les éléments de la troisième et de la cinquième colonne ne sont jamais en cooccurrence ( $c f$. Miller/Monachesi 2003, Miller/Sag 1997, Morin 1981, Perlmutter 1971, Pomino 2009 pour une discussion approfondie). Les proclitiques des colonnes 3 à 7 peuvent se placer entre ne et le verbe conjugué. Dans le diagramme (67) figurent les résultats des quatre études qui ont pris en considération l'influence de ces proclitiques de manière détaillée. Les clitiques figurent dans la légende du diagramme 
(67) selon leur ordre préverbal. Les chiffres 3 à 7 indiquent la colonne dans laquelle l'élément respectif apparaît dans le tableau (66).

(67) L'influence des proclitiques non-sujets sur $\pm n e$

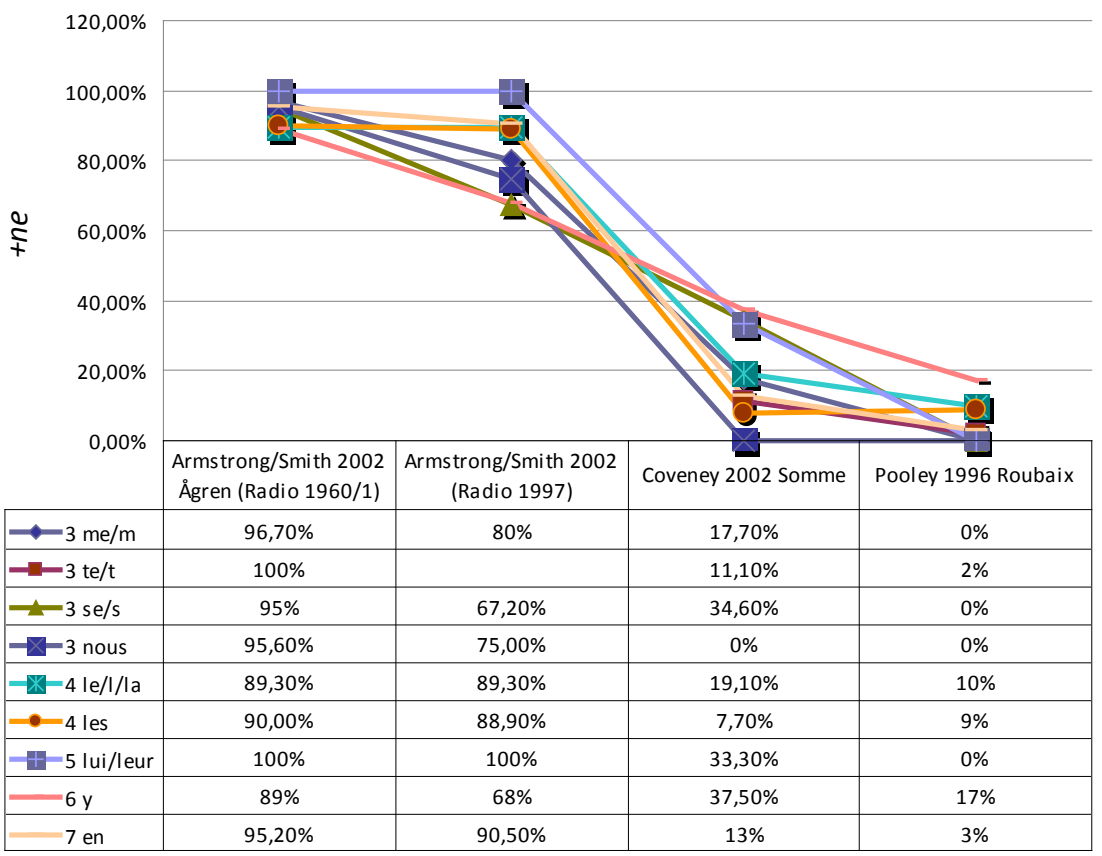

Nous tenons à signaler que certains pourcentages du diagramme (67) se basent sur un nombre assez restreint de cas et ne sont pas statistiquement robustes. Ceci dit, il saute à l'œil que les lignes forment une bande compacte et que tous les taux reculent en diachronie et en fonction de la nature des corpus.

Dans les deux corpus radiophoniques analysés par Armstrong/ Smith (2002), les taux de +ne sont relativement élevés avec tous les proclitiques, ce qui est sans doute dû à la nature formelle des corpus. Surtout dans le corpus d'Ågren (1973), analysé par Armstrong/Smith (2002), il n'y a quasiment aucune différence d'un clitique à l'autre. Les légères différences entre les proclitiques surgissent uniquement dans 
les corpus plus récents. Dans la communication directe, analysée par Coveney (2002) et Pooley (1996), les chiffres sont beaucoup plus restreints et se situent entre $0 \%+n e(n o u s)$ et $37,5 \%+n e(y)$ dans le premier corpus, et entre $0 \%+n e$ (me, te, nous, lui/leur) et $17 \%+n e(y)$ dans le second. Nous observons que la plupart des éléments subissent une régression brusque lorsque l'on passe des corpus radiophoniques aux corpus de communication directe. Seul le clitique locatif $y$ montre une descente linéaire des taux de $+n e$ : tandis que dans les corpus radiophoniques il manifeste les taux de $+n e$ les plus restreints, dans la communication directe, il semble ralentir, plus que d'autres proclitiques, la chute de ne. Toutefois, dans l'ensemble, les proclitiques non-sujets ne semblent pas avoir une influence claire sur \pm ne.

(68) Conclusions concernant l'influence d'autres proclitiques

\begin{tabular}{|lll|}
\hline & $+n e$ & $-n e$ \\
\hline Armstrong/Smith (2002: & - & - \\
28), Ashby (1981: 679) & & \\
Coveney (22002: 76-77) & avec d'autres proclitiques & sans d'autres proclitiques \\
Pooley (1996: 174) & $l e, l, l a, y$ & - \\
Sturm (1981: 134-135) & - & avec un sujet lexical ou avec qui \\
\hline
\end{tabular}

Coveney (22002: 76-77) constate que la réalisation de ne est légèrement (plus?) élevée dans les contextes contenant des proclitiques. Pooley (1996: 174), quant à lui, suggère que $l e, l$, la (quatrième colonne) et $y$ (sixième colonne) peuvent favoriser l'occurrence de ne. Armstrong/ Smith (2002: 39) ainsi qu'Ashby (1981: 679) ne signalent aucune différence notable entre les contextes proclitiques non-sujet et les corpus globaux. Pour sa part, Sturm (1981: 134-135) fait noter qu'en présence des proclitiques non-sujet il y a une tendance à omettre ne si le sujet est un lexème ou le pronom relatif qui.

Malgré le fait que l'évidence empirique de l'influence des proclitiques non-sujet sur la réalisation de ne est plutôt pauvre, ce sujet est au cœur de l'une des thèses qui exliquent la variation $\pm n e$ ( $c f$. Posner 1985 : 189 et Larrivée 2014 présentée dans la section 2.3.2) et sera repris dans la discussion d'une explication prosodique de $\pm n e$ en section 5.2.4.2. 


\subsubsection{L'élément négatif non-clitique}

Le diagramme (69) montre l'état des lieux quant à l'influence de l'élément négatif non-clitique sur la réalisation de $n e$. Les taux des différents éléments négatifs produisent des lignes assez parallèles à travers les corpus étudiés. Seul le pronom indéfini personne constitue une exception à cette généralisation, mais ses oscillations sont largement dues à sa fréquence réduite dans la majorité des corpus ( $c f$. aussi Coveney 1998: 164).

(69) L'influence du terme négatif sur $\pm n e^{49}$

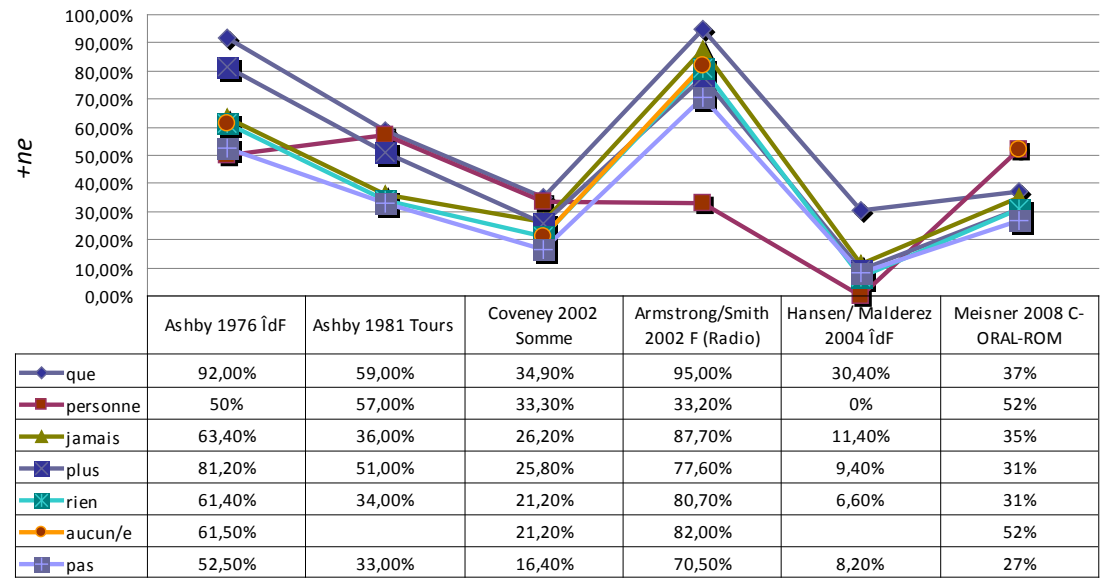

Contrairement aux différents types de sujet, les lignes représentant les éléments négatifs forment une bande solide au centre du diagramme (69). Ceci signifie qu'il n'y a aucun élément négatif qui semble particulièrement inciter ou empêcher la réalisation de ne. La seule demiexception à cette règle est constituée par l'élément restrictif que, qui se situe (dans toutes les analyses, sauf dans la dernière, celle de Meisner 2010) légèrement au-dessus des autres termes négatifs. Pour l'analyse

49 Chiffres dans le diagramme: cf. Ashby (1976) cité d'après Coveney (1998: 165); Ashby (1981: 682); Coveney (2002: 76); Hansen/Malderez (2004: 22); Armstrong/Smith (2002: 37) et Meisner (2008: 62). 
de ce facteur dans notre corpus ( $c f$. section 4.4.3), nous ne nousattendons donc à aucune influence indépendante sur la variable $\pm n e$. Il semble plus probable que les légères différences observables entre les différents éléments négatifs soient provoquées par leurs fréquences variables: étant largement plus fréquent que les autres éléments ( $c f$. aussi section 4.4.3), pas est éventuellement plus susceptible d'apparaître dans des séquences préformées sans ne (cf. Moreau 1986).

\subsubsection{Les temps et modes verbaux}

Dans le diagramme (70) figure un résumé de l'influence du temps verbal sur \pm ne trouvée dans trois analyses de corpus. Comme dans les autres diagrammes, les taux de ne varient en fonction des corpus.

(70) L'influence du temps verbal sur $\pm n e$

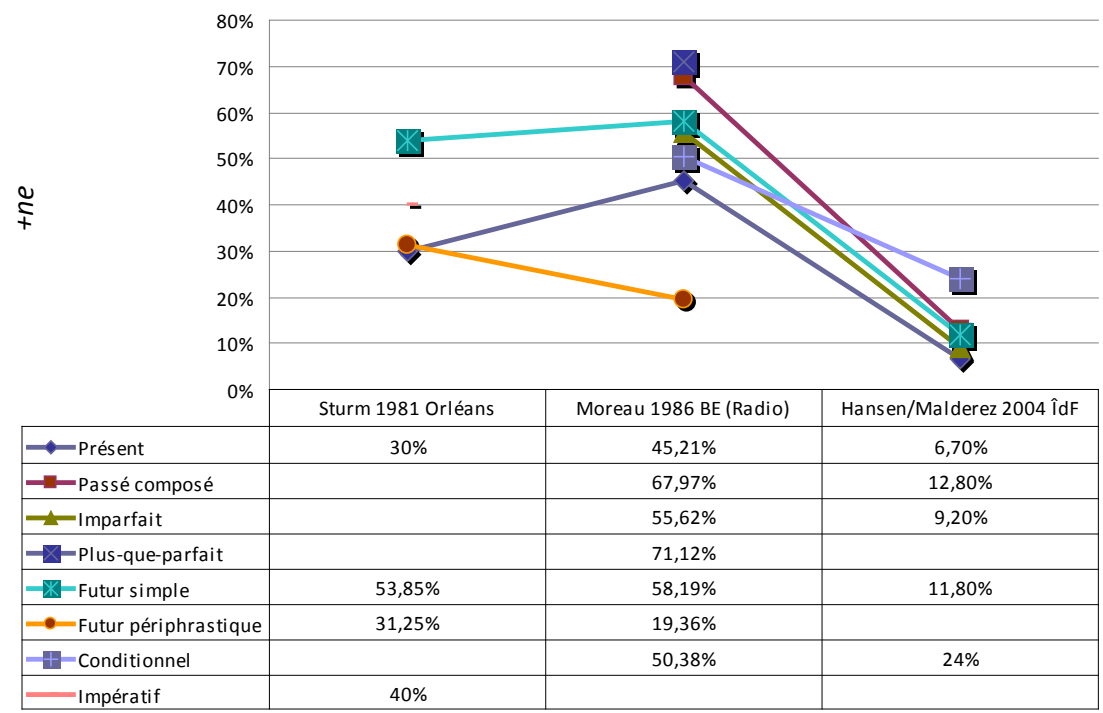

Nous observons des taux constamment bas avec le présent et le futur périphrastique (surtout dans le corpus de Moreau 1986), tandis que le futur simple et le conditionnel manifestent des taux plus élevés. Les différences entre les temps verbaux semblent être plus articulées dans 
les corpus de Sturm (1981) et de Moreau (1986) que dans celui plus récent de Hansen/Malderez (2004). Tandis qu'Ashby (1981: 680) soutient que ne est réalisé catégoriquement avec l'impératif, l'étude de Sturm (1981) montre que ceci n'est pas correct: il n'observe que $40 \%$ +ne dans les impératives.

D’une manière générale, les lignes du diagramme (70) forment une bande assez compacte, ce qui signifie que les différences parmi les temps verbaux ne sont pas considérables. Comme l'élément négatif non-clitique, les temps et modes verbaux (testés dans notre analyse de corpus en section 4.4.4.4) ne se prêtent donc guère à une explication autonome de la variation $\pm n e$. Il semble plus probable qu'ils exercent une influence indirecte en tant que contexte phonologique suivant la variable $\pm n e$ ( $c f$. les sections 2.2 .5 .7 et 4.4.6) et à travers leurs fréquences respectives.

\subsubsection{Le type de phrase: l'opposition entre principale et subordonnée}

Au niveau syntaxique, Ashby (1976: 125, $1981: 678)$ et Sturm (1981: 62) ont testé l'influence du type de phrase, plus précisément l'influence de l'opposition entre principales et subordonnées sur $\pm n e$. Les résultats de ces études figurent en diagramme (71).

(71) L'influence du type de phrase sur \pm ne

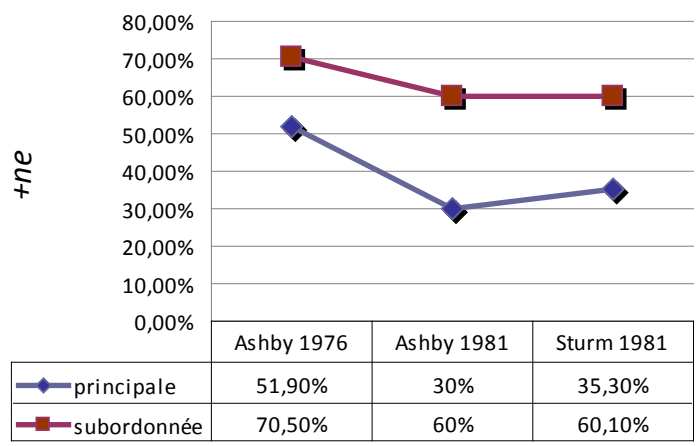

Les données en (71) suggèrent de manière relativement claire que la réalisation de ne est plus probable dans les subordonnées que dans les 
principales ${ }^{50}$. Tandis que les principales montrent des taux moyens de $+n e$ entre $30 \%$ et $51,9 \%+n e$, la réalisation de la particule affiche des taux compris entre $60 \%$ et $70,5 \%+n e$ dans les subordonnées ( $c f$. Ashby 1976: 125, 1981: 678). Ashby (1981: 679) soupçonne que ce résultat reflète l'hypothèse de Givón (1976: 170), selon laquelle la phrase principale serait l'environnement le plus innovateur et progressif et qu'un changement linguistique s'étendrait d'ici vers d'autres contextes syntaxiques. Sturm (1981: 62-63) fait noter que ce résultat pourrait être lié au fait que certaines subordonnées contiennent un verbe au subjonctif, ce qui favoriserait la réalisation de ne. Toutefois, Ashby (1981: 678) compare les taux de différents types de subordonnées et observe que celles au subjonctif, qui montrent un taux de $65 \%+n e$, ne se distinguent pas considérablement des autres types de subordonnées. En ce qui concerne les différents types de subordonnées, les taux respectifs de + ne figurent dans le diagramme (72).

(72) L'influence du type de subordonnée sur $\pm n e$ (cf. Ashby 1976: 125, $1981: 678$ )

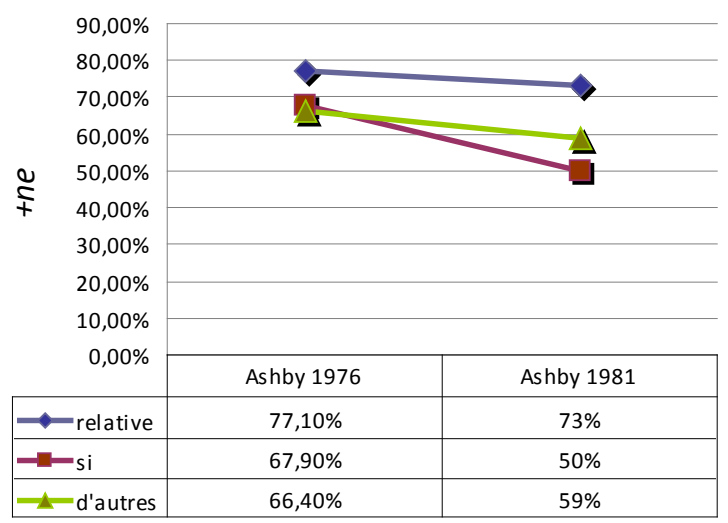

La réalisation de ne dans les subordonnées est généralement élevée par rapport aux principales. La comparaison des différents types de subordonnées en (72) permet de découvrir une variation subtile: apparem-

50 Lüdicke (1982: 48) arrive au même résultat, mais ne publie pas les taux exacts trouvés dans son corpus pour les principales et les subordonnées. 
ment, dans les relatives, la réalisation de ne est encore plus stable que dans les phrases en si et dans les autres types de subordonnées.

Nous vérifierons donc si l'opposition principale vs. subordonnée ainsi que le petit décalage entre les relatives et d'autres types de subordonnées est également observable dans notre corpus ( $c f$. section 4.4.5.1). De plus, ce facteur sera lui aussi intégré dans l'explication prosodique de la variable $\pm n e$ ( $c f$. section 5.2.4.2).

\subsubsection{Les influences phonologiques}

Les facteurs phonologiques, dont l'influence sur \pm ne a été testée dans les analyses de corpus, concernent avant tout le contexte phonologique directement adjacent à ne. Le seul facteur en relation avec le niveau suprasegmental, testé par Ashby (1976: 128), est la vitesse de la production langagière: en débit rapide (360-580 syllabes par minute), les locuteurs omettent environ $10 \%$ plus de ne qu'en langage réfléchi (100340 syllabes par minute).

Au niveau segmental, les locuteurs essaient apparemment d'éviter les hiatus: en position intervocalique, ne est souvent réalisé ( $c f$. Ashby 1976: 129), surtout si l'une des deux voyelles est nasale ( $c f$. Ashby 1981: 677). La position postpausale, surtout avant une voyelle, incite elle aussi à la réalisation de ne ( $c f$. Ashby 1981: 677). Lüdicke (1982: 45) remet en question l'interdépendance entre le contexte phonétique suivant ne et le type de verbe: effectivement, la fréquence des ne prévocaliques est beaucoup plus élevée avec les auxiliaires avoir et être qu'avec les verbes lexicaux.

Coveney (1998: 167) thématise une autre difficulté de l'analyse des facteurs phonologiques. Il fait noter qu'au lieu d'être un produit du contexte phonologique, l'absence ou la présence de la variable ne le détermine souvent:

(73) the chief difficulty is as follows: in the very large number of tokens where $j e$ or $c e$ is the subject, and the word following the locus for (ne) begins with a vowel, the preceding phonological environment is different according to whether $n e$ is present or absent: if $n e$ is present, the preceding segment is usually the schwa, but if it is absent, the schwa is elided, and the preceding 
segment is the consonant. It seems therefore that in these cases (which are very numerous in any given corpus) the variable is affecting the phonological environment, rather than vice versa. (Coveney 1998: 167)

La citation de Coveney (1998: 167) montre qu'il y a une grande interdépendance entre la présence ou l'absence de la variable ne même et le caractère de son contexte phonologique. De plus, il semble clair que l'absence du ne est inévitablement liée à l'absence du schwa ${ }^{51}$, donc au noyau vocalique de la syllabe atone ne (cf. Coveney ${ }^{2} 2002$ : 77-78). Étant obligatoirement élidé avant une voyelle à l'intérieur d'un groupe accentué ( $c f$. (74)a), le schwa du ne a tendance à tomber également dans des contextes consonantiques ( $c f$. (74)b). Sans voyelle, la consonne [n] ne forme aucune syllabe complète et est intégrée dans l'une des syllabes adjacentes: dans une attaque simple, comme en (74)a, ou dans la coda, comme en (74)b.

(74) Les conséquences de l'omission du schwa pour la structure syllabique

a. Je n'aime pas $\rightarrow$ [3ə.ncm.pa] ([n] dans l'attaque simple)

b. Je ne sais pas $\rightarrow$ [3ən.se.pa] ([n] dans la coda)

c. je ne veux pas $\rightarrow$ ? [3(n)vø.pa] ([n] dans une attaque complexe)

d. je ne le veux pas $\rightarrow{ }^{*}[3(\mathrm{n})$ lvø.pa] ([n] dans une attaque complexe)

Le schwa forme également le noyau d'autres clitiques ${ }^{52}$ adjacents à ne comme je, ce, me, te et le. C'est la disparition du schwa de ces clitiques qui défavorise la réalisation de $n e$, car sans schwa, ils donnent lieu à des séquences consonantiques complexes, $c f$. (74)c, voire imprononçables, $c f$. (74)d, qui peuvent à leur tour être réduites si ne est omis.

Finalement, l'hypothèse selon laquelle l'omission de ne est moins accentuée au sud de la zone francophone ( $c f$. section 2.2.4.1) suit la même ligne que les études phonologiques qui montrent une réalisation plus stable du schwa au sud de la France (cf. Pustka/Eychenne 2007). En d'autres termes: si le schwa est plus stable, ne est plus stable. Nous

51 La notion de schwa désigne l'alternance entre [œ], [ø] ou [ə] et zéro, comme par exemple dans belle fille, réalisé au nord de la France plutôt comme [belfij] et au sud plutôt comme [beləfijə] (cf. Pustka/Eychenne 2007).

52 Nous tenons à noter que l'instabilité vocalique concerne également, mais à un moindre degré, la voyelle [y] dans $t u$. 
pouvons donc assumer que la réalisation du schwa joue un rôle crucial pour la réalisation de $n e$.

Pour conclure cette section, nous constatons que, même si les influences phonologiques sont généralement reconnues comme cruciales pour la réalisation de $n e$ ( $c f$., entre autres, Ashby 1976: 128-129 et 1981: 677, Coveney 1998: 167 et $^{2} 2002$ : 77-78 et Lüdicke 1982: 45), leur analyse, surtout au niveau suprasegmental, est méthodologiquement pénible et leur interprétation reste difficile et demeure souvent sans résultats.

Dans notre analyse, nous accorderons une attention particulière à ce facteur en testant non seulement l'influence du contexte segmental sur la variable $\pm n e$ ( $c f$. section 4.4.6), mais aussi celle du contexte suprasegmental, en particulier celle du poids phonologique du type de sujet ( $c f$. section 4.4.1), des séquences proclitiques ( $c f$. section 4.4.2) et de la forme verbale ( $c f$. section 4.4.5). De plus, la prosodie sera au cœur de l'explication de la variation \pm ne que nous proposerons en section 5.2.

\subsubsection{Discussion critique des analyses empiriques de \pm ne}

Même si les analyses de corpus des dernières décennies ( $c f$. Armstrong/ Smith 2002, Ashby 1976, 1981, 2001, Coveney 22002, Hansen/Malderez 2004 etc.) ont mis à jour une quantité considérable d'aspects partiels concernant la distribution de \pm ne en français contemporain, les méthodes appliquées restent perfectibles et un regroupement de tous les faits observés est toujours attendu.

Dans ce sens, Gadet (2000: 164-165) formule une critique presque dévastatrice lors d'une synthèse de nombreuses études de l'omission de ne. Elle observe en particulier les points suivants :

(75) Les points critiques des analyses de \pm ne selon Gadet (2000: 164-165)

a. L'absence des études contrastives diatopiques et diaphasiques

b. La pauvre variation diastratique

c. Le manque de créativité quant à la collection des données

d. L'absence de confrontation avec les résultats d'autres chercheurs

e. La pauvre volonté [...] d'interpréter les statistiques

f. La confusion générale entre corrélation et explication 
Il est vrai que les études contrastives diatopiques et diaphasiques sont relativement rares, comme affirmé en (75)a. L'absence des études contrastives serait moins problématique si les comparaisons entre différentes analyses étaient plus faciles. Or, les définitions d'une situation 'formelle' ou 'informelle' varient considérablement dans la pratique sociolinguistique (ceci vaut également pour les 'classes sociales' et les groupes d'âge). Il faut ajouter que, à l'exception du facteur diatopique, les facteurs extralinguistiques (jeune - âgé, privilégié - non-privilégié et formel - informel) ont l'inconvénient méthodique d'être graduables et difficiles à concevoir.

En revanche, il existe quelques études contrastives diasituationnelles, comme par exemple celle d'Armstrong (2002). Malheureusement, les situations sélectionnées dans cette étude s'avèrent inadéquates pour décrire la variation subtile présente dans les données. Ceci signifie que les méthodes peuvent alors être affinées en ce qui concerne ce domaine.

Gadet (2000: 164-165) ajoute en (75)b et c par rapport aux individus étudiés: «il semble que l'objet d'étude préféré des linguistes est leur semblable». En effet, ce sont surtout des intellectuels qui sont enregistrés pendant les interviews sociolinguistiques, ce qui est dû sans doute à la difficulté d'établir des contacts avec des sujets parlants socialement très éloignés des chercheurs mêmes et de les enregistrer pendant des interactions naturelles ( $c f$. la discussion du paradoxe de l'observateur en section 3.6).

En (75)d et e Gadet (2000: 164-165) se plaint de «l'absence» de comparaisons avec d'autres études («le seul qui le fait est Sturm (1981), qui, à son tour, n'est pas cité par les linguistes français») et de «la pauvre volonté [...] d'interpréter les statistiques»».

Nous admettons que, dans les analyses statistiques, les influences linguistiques sur \pm ne se lisent généralement comme une longue liste de facteurs plus ou moins indépendants lesuns des autres ( $c f$. par exemple le tableau de plus d'une page dans Ashby 1981 : 678-679 et la discussion de son approche dans Lüdicke 1981: 44-45). Les cooccurrences fréquentes ainsi que les interdépendances entre certaines variantes ayant une influence sur \pm ne restent largement négligées dans la majorité des 
travaux à ce sujet ( $c f$. par exemple Ashby 1976, 1981, 2001, Coveney 2002). En revanche, Lüdicke (1982) se concentre explicitement sur l'interdépendance entre les attaques verbales et le contexte phonologique de $\pm n e$, et Moreau (1986) ainsi que Sturm (1981: 136-151) analysent les tendances de lexicalisation dans les 'séquences préformées' de haute fréquence.

Quant à la «confusion générale entre corrélation et explication» citée en (75)f, nous supposons que les moyens statistiques modernes incitent éventuellement à la pure constatation de corrélations significatives, sans pour autant dédier assez d'espace à leur discussion (par exemple, dans Ashby 1981 : 677-686 dix pages d'analyse statistique sont suivies par dix lignes de conclusions). Par conséquent, les interactions entre les facteurs intra- et extralinguistiques pourraient être elles aussi mieux exploitées: par exemple, Armstrong/Smith (2002: 30-34) et Dufter/ Stark (2007 : 120) vont dans cette direction en essayant de lier l'omission de $n e$ à la fréquence respective des types de sujet dans les corpus.

\subsubsection{Conclusion intermédiaire: quelques implications} pour notre analyse de corpus

Que pouvons-nous alors conclure de la discussion des études empiriques de \pm ne pour la conception de notre analyse de corpus dans le chapitre 4 ?

Nous retenons de la critique de Gadet (2000) certains points importants qu'il faudra respecter:

(76) Points importants pour la conception de l'analyse de corpus

a. Focus contrastif diaphasique et diatopique

b. Données authentiques de situations naturelles

c. Conception des facteurs linguistiques comme 'réseau'

d. Nouvelle classification des types de sujet

e. Analyse exploratrice, descriptive et multifactorielle

f. Évaluation des hypothèses existantes

g. Interprétation des résultats

h. Proposition d'une explication prosodique 
Selon Gadet (2000), le fait de tester au hasard l'influence d'une grande quantité de facteurs sur la variable $\pm n e$ n'est pas suffisant. Par rapport aux points (76)a et $\mathrm{b}$, nous nous concentrerons, par conséquent, en particulier sur l'axe intrapersonnel et diatopique en analysant des données spontanées et naturelles.

Lors de l'analyse de corpus, nous tacheronsd'interpréter les interactions entre les variables pertinentes pour \pm ne en les concevant en tant qu'un 'réseau complexe' d'influences potentiellement interdépendantes, comme illustré en (76)c.

En (77) figure un schéma de ce réseau: les influences sur \pm ne apparaissent en orange, les variantes qui favorisent sa réalisation en vert et celles qui favorisent son absence en bordeau.

(77) Réseau d'influences linguistiques sur ne

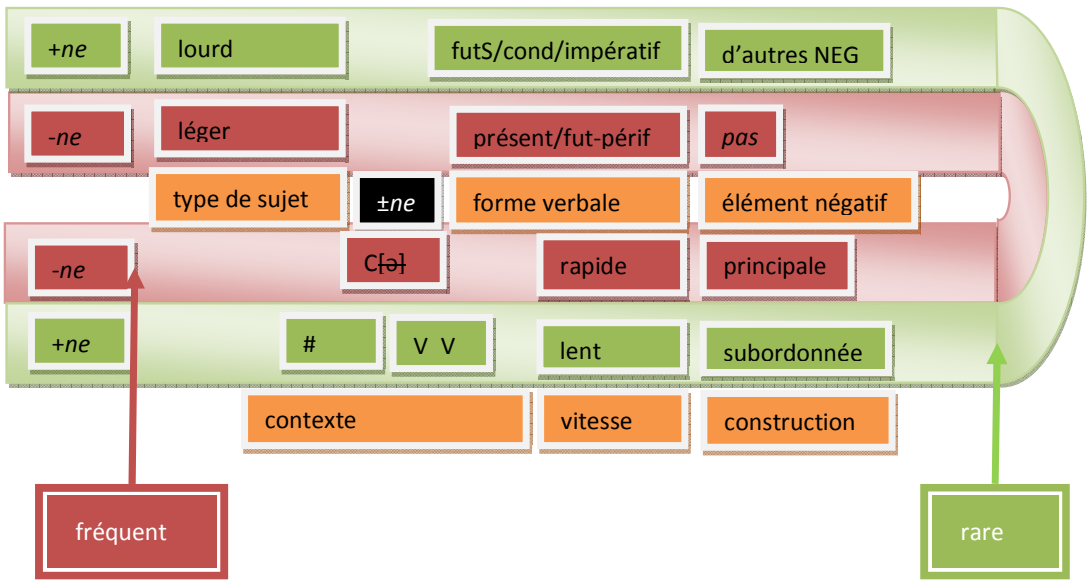

$\mathrm{Au}$ centre de la figure (77) se trouve la variable \pm ne (en noir), entourée par les facteurs qui forment son contexte morphosyntaxique et phonologique direct (en orange), à savoir le type de sujet à gauche de la variable $\pm n e$ et la forme verbale, suivie par l'élément négatif à droite ${ }^{53}$.

53 Les autres clitiques ne figurent pas dans le schéma, car leur influence n'est pas clairement déterminée. 
Les influences moins directes, comme le débit de parole et la construction syntaxique, figurent en dessous (en orange). Dans la partie inférieure du schéma (77) figure le métafacteur de la fréquence, qui conditionne dans le discours le poids respectif de tous les autres facteurs: les variantes plus fréquentes apparaissent sur fond rouge et celles plus rares surfond vert.

Dans l'ensemble, les variantes fréquentes favorisent l'absence de ne (les sujets clitiques, les formes verbales au présent, l'élément négatif pas, la phrase principale, le débit de parole et l'élision du schwa), tandis que les variantes rares favorisent sa réalisation (les sujets nonclitiques, les formes verbales du futur simple, du conditionnel et de l'impératif, des éléments négatifs autres que pas, la phrase subordonnée, l'articulation lente et le contexte phonologique vocalique et postpausal).

De plus, en (78) figure une série d'interdépendances linguistiques entre les variantes qui méritent d'être considérées.

(78) Interdépendances entre les facteurs linguistiques
a. Sujet je, ce
$\rightarrow$ (souvent) $\mathrm{C}[\mathrm{O}$
b. Sujet nous, vous $\rightarrow \mathrm{V}_{-}$
c. Passé composé avoir $\rightarrow \overline{\mathrm{V}}$
d. Impératif $\rightarrow$ \#
e. Futur périphrastique $\rightarrow$ (souvent)_C
f. personne, rien (sujet) $\rightarrow$ sujet non-clitique
g. Débit rapide
$\rightarrow$ (souvent) $\mathrm{C}$ [o]_

Les clitiques sujet je et ce contiennent un schwa qui est souvent élidé (cf. (78)a), surtout si le débit de parole est élevé (cf. (78)g), ce qui peut inciter à l'omission de $n e^{54}$. Les sujets nous et vous donnent toujours lieu à un contexte vocalique gauche $(c f$. (78)b), tandis que le passé composé avec l'auxiliaire avoir forme un contexte vocalique droit $(c f .(78) c)$ (ceci vaut aussi pour certaines formes de l'auxiliaire être: es, est et êtes), et avec l'impératif, \pm ne apparaît souvent au début d'un énoncé dans un contexte postpausal ( $c f . \pm$ ne chantez pas!, (78)d). Comme

54 Ceci peut valoir également pour tu: contrairement au [u] dans nous et vous, le [y] dans $t u$ peut être élidé comme un schwa. 
nous l'avons vu, ces contextes intervocaliques ou suivant une pause favorisent la présence de ne. Finalement, les éléments négatifs personne et rien peuvent apparaître en position de sujet, évidemment nonclitique, d'une phrase négative (cf. (78)f). Nous essayerons de tenir compte de ces interdépendances lors de notre analyse.

Retournons maintenant aux autres points retenus pour l'analyse de corpus en (76). En ce qui concerne le point d, nous avons de bonnes raisons (montrées dans la section 2.2.5.2) d'appliquer une classification révisée des types de sujet, qui représenteplus clairement les influences sur $\pm n e$ et les propriétés morphophonologiques des éléments en question que la simple distinction entresujets pronominaux et lexicaux:

(79) Classification des types de sujet

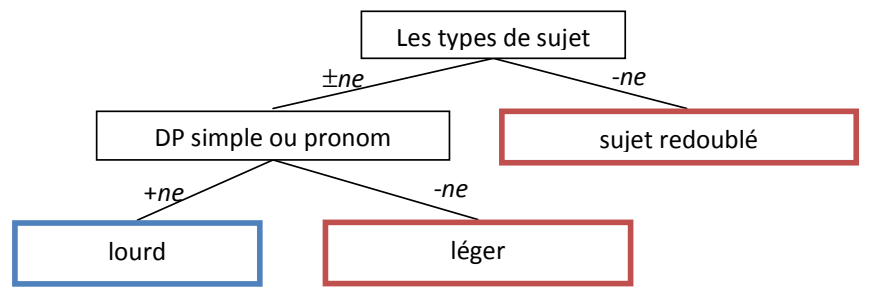

Cette classification tripartite des types de sujets en des sujets lourds, qui favorisent la présence de ne, et des sujets légers et redoublés, qui favorisent son absence, constituera la base de l'analyse qui suit.

Comme mentionné en (76)e, nous exécuterons non seulement une analyse descriptive détaillée de la variable $\pm n e$, comme l'ont déjà fait généralement les analyses précédentes, mais aussi une analyse exploratrice, qui regroupera les locuteurs en trois catégories manifestant des comportements similaires quant à la variable $\pm n e$, ainsi qu'une analyse statistique multifactorielle, qui permettra de mesurer le poids respectif des variables et leurs interactions pertinentes.

Après l'analyse, et comme annoncé en (76)f, les hypothèses existantes quant à la variation $\pm n e$ seront évaluées à l'aide des résultats issus de nos données, et finalement, l'ensemble des résultats disponibles sera discuté et réinterprété, ce qui donnera lieu à la proposition d'une explication prosodique de la variation $\pm n e$ ( $c f$. (76)g et $\mathrm{h}$ ). 
Cependant, avant de passer à l'analyse même, nous présenterons dans la section qui suit les approches théoriques de la variable $\pm n e$, que nous évaluerons à travers nos données.

\subsection{Les approches de la variation $\pm n e$}

Certains résultats des analyses des corpus phoniques, résumés dans les sections précédentes, sont repris directement ou indirectement par quatre hypothèses explicatives de la variable $\pm n e$, qui seront discutées dans les sections suivantes. Il s'agit premièrement de l'approche sociolinguistique ( $c f$. entre autres Ashby 1976, 1981, 2001, Gadet 1997b, Coveney ${ }^{2} 2002$ : 29-54 et Labov 1972a,b), qui soutient que \pm ne serait en covariation stable avec des facteurs extralinguistiques ( $c f$. section 2.3.1). Deuxièmement, nous aborderons l'approche interne ( $c f$. Larrivée 2014, Posner 1985), selon laquelle plusieurs proclitiques s'excluraient mutuellement ( $c f$. section 2.3.2). Troisièmement, l'approche diglossique (cf. Culbertson 2010, Massot 2010, Zribi-Hertz 2011 et Koch/Oesterreicher ${ }^{2} 2011$ ) soutient que les locuteurs alternent continuellement entre deux grammaires du français, dont l'une contient la particule ne et l'autre non ( $c f$. section 2.3.3). Finalement, la quatrième approche ( $c f$. van Compernolle 2008b, 2009, Fonseca-Greber 2007, Sankoff/Vincent 1980, Poplack/St-Amand 2009) analyse ne en tant que particule pragmatique, éventuellement comparable à la particule en du flamand (cf. Breitbarth/Haegeman 2010 et la section 2.3.4).

\subsubsection{L'approche externe: \pm ne comme variable sociolinguistique}

La sociolinguistique a découvert que les variantes linguistiques dites 'libres' sont en réalité souvent conditionnées par des facteurs extralinguistiques. Une variable sociolinguistique (souvent phonologique) est conçue comme ayant une fréquence élevée, étant à l'abri de toute 
maîtrise consciente du locuteur, faisant partie d'une structure plus large et étant quantifiable sur une échelle linéaire ( $c f$. Labov 1972b: 8, Gadet 1997b: 5). Elle se trouve en corrélation stable avec certains groupes de locuteurs, définis par des facteurs extralinguistiques comme l'âge ou la classe sociale ( $c f$. Armstrong 2001, Beeching et al. édd. 2009, Gadet 1997b). Un exemple célèbre est la stratification sociale de la prononciation du /r/ en anglais ( $c f$. Labov 1972b).

Les tentatives d'étendre la méthodologie variationniste de la phonologie aux autres niveaux du système linguistique se sont avérées difficiles, surtout en ce qui concerne la syntaxe et la morphosyntaxe (cf. Coveney ${ }^{2} 2002$ : 29-54 et Gadet 1997b pour une discussion). La variable $\pm n e$, qui, de la même façon que les variables phonologiques, comprend deux variantes ( $+n e$ et $-n e$ ) qui n'opèrent aucune modification sémantique, est l'un des rares phénomènes hors de la phonologie qui se prête à une démarche sociolinguistique.

Les premières grandes études sociolinguistiques du $20^{\mathrm{e}}$ siècle basées sur des corpus oraux confirment que l'usage de ne varie effectivement à travers la société ( $c f$. section 2.2.4). Toutefois, il reste peu clair si \pm ne est vraiment une variable sociolinguistique en synchronie et, si c'est le cas, de quel type, $c f$. (80). Les indicateurs montrent une stratification sociale mais pas stylistique, les marqueurs, quant à eux, montrent une stratification aussi bien sociale que stylistique et finalement, les stéréotypes sont des marqueurs qui ont gagné l'attention consciente des locuteurs et ne fonctionnent donc plus comme des variables sociolinguistiques discrètes. Les flèches indiquent la direction habituelle de l'évolution entre les différents types.

(80) Les fonctions d'une variable sociolinguistique (cf. Trudgill 2003: 63, 85, 129)

\begin{tabular}{|l|l|l|l|}
\hline & indicateur $\rightarrow$ & marqueur $\rightarrow$ & stéréotype \\
\hline Stratification sociale & + & + & + \\
\hline Stratification stylistique & - & + & + \\
\hline Attention consciente & - & - & + \\
\hline
\end{tabular}

Coveney (2002: 82) conclut que, contrairement au sexe et à la classe sociale des locuteurs, l'âge serait le seul critère de groupe déterminable 
de façon fiable à l'aide de la variable \pm ne. Tandis qu'Ashby (1976: 133) ne trouve aucune différence notable entre les jeunes et les adultes, l'importance du facteur de l'âge est soutenue par Ashby (1981: 682), qui trouve ultérieurement que l'absence de ne serait plus diffusée parmi les ouvriers que parmi la classe moyenne et élevée. Ces différences entre les classes sociales seraient interprétables en termes d'un changement 'from below'.

Si l'on veut décrire $\pm n e$ en termes du schéma cité en (80), la fonction la plus probable de \pm ne serait celle d'un marqueur. Par contre, la variable n'est pas un indicateur, car l'importance stylistique semble désormais plus importante que celle sociale ( $c f$. aussi les discussions dans Coveney ${ }^{2} 2002$ : 84-85 et en section 2.3.4). Elle ne semble pas non plus un stéréotype, car les deux variantes se trouvent sans difficultés dans tous les corpus phoniques. Dans ce contexte, Coveney (2002: 58) observe: «Whilst ne can clearly be regarded, then, as the more prestigious variant, its absence from informal styles does not seem today to be subject to any stigmatization ». Pourtant, il y a des indices qui montrent que les deux variantes pourraient être stigmatisées dans certains contextes. Ainsi, Meisner (2013) observe, par exemple, que dans les situations intimes entre jeunes, les séquences clitique sujet (léger) + ne ainsi que l'omission de la particule avec un sujet lexical dans une situation de distance, comme par exemple un exposé en classe, sont inacceptables. Nous discuterons en section 5.1.1 si les données de notre corpus permettent la classification de $\pm n e$ comme une variable sociolinguistique et si ceci est le cas, de quel type de variable sociolinguistique il s'agit.

\subsubsection{L'approche interne: l'hypothèse de l'incompatibilité entre ne et d'autres clitiques}

Selon une hypothèse formulée par Posner (1985: 189) et reprise par Larrivée (2014), les séquences clitiques du type (81)a posent des problèmes articulatoires aux locuteurs, ce qui pourrait même donner lieu à une 'contrainte clitique' qui exclurait l'élément le plus faible de la séquence ( $c f$. à cet égard aussi les idées de Culbertson 2010, Culbertson/ 
Legendre 2008 et Massot 2010 décrites dans la section suivante). Ceci serait notamment le cas du clitique de négation ne, car il est sémantiquement superflu.

(81) \pm ne dans les séquences clitiques
a. $j$
$\begin{array}{rll}n e & \text { le } & \text { lui } \\ \text { I-1S-NOM NEG } & \text { 3S-ACC } & \text { 3s-DAT }\end{array}$
ai pas donné
b. $\quad[3 ə$.
nว. lə.
lчі.
have NEG given
c. [зчі.
$\varepsilon$. pa. do.ne] articulation prudente
$\varepsilon$. pa.do.ne] articulation rapide

Tandis qu'une articulation prudente telle qu'en (81)b permet la réalisation de jusqu'à quatre clitiques consécutifs, dont chacun correspond à une syllabe, les clitiques sujet et objet se fusionnent dans les séquences complexes du langage spontané et rapide. Les éléments sont souvent réduits à une seule syllabe, comme celle en $(81) \mathrm{c}$, et ne (dans cet exemple également le) disparaît sans laisser de trace. Ce sont donc, en premier lieu, des raisons phonotactiques qui empêchent la réalisation des séquences clitiques complexes. Le patron de variation typiquement observé dans les analyses de corpus est celui présenté en (3) et repris en (82).

(82) Patron de variation \pm ne typique selon le type de sujet

\begin{tabular}{|l|l|l|}
\hline Sujet & \multicolumn{1}{l}{ \pm Exemple du corpus } \\
\hline -clitique & $+n e$ & a. Ulisse ne montre aucun intérêt dans le dialogue (0046) \\
\hline +clitique & $-n e$ & b. $\boldsymbol{j}$ 'ai pas grand chose à dire (0134) \\
\hline
\end{tabular}

Afin de modeler la variation illustrée en (82), l'on pourrait proposer une règle syntaxique qui élimine la particule ne après les sujets clitiques (cf. (83)) ou bien une règle d'insertion de ne après les sujets nonclitiques.

(83) Règle d'élimination de $n e$ : sujet +clitique $\rightarrow-n e$

(84) Règle d'insertion de $n e: \quad$ sujet-clitique $\rightarrow+n e$

Une règle d'élimination semblable à celle en (83) a été proposée par plusieurs syntacticiens par rapport aux discours informels ( $c f$. Dubois

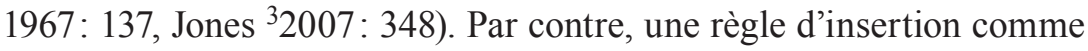


celle en (84) a été revendiquée (par exemple par Fonseca Greber 2007) face aux taux réduits de ne dans certains corpus, mais n'a jamais été formalisée. Ce manque de formalisation s'explique essentiellement par le problème de motiver au niveau théorique l'insertion d'un élément quelconque: d'un point de vue génératif, l'élimination phonologique d'un élément qui est sous-jacent en syntaxe est généralement plus économique et donc favorable à l'insertion d'un élément ${ }^{55}$. Nous discuterons en section 5.1.2 si une telle incompatibilité entre les clitiques sujet et ne peut être soutenue par les données de notre corpus.

\subsubsection{L'approche diglossique: l'hypothèse des deux grammaires du français}

En linguistique variationnelle, une langue historique est conçue comme un espace variationnel complexe contenant des variétés diatopiques, diastratiques, diaphasiques et éventuellement entre l'immédiat et la distance communicative ( $c f$. Flydal 1951, Coseriu 1988a,b, Koch/Oesterreicher ${ }^{2} 2011$ ).

Récemment, la variation intrapersonnelle observée chez les francophones a été formalisée dans une optique similaire. Les modèles de Massot (2010), Mensching (2008) (dans une conception minimaliste, comprenant deux lexiques) et Zribi-Hertz (2011) ont en commun avec les analyses variationnelles le fait qu'ils attribuent une série de phénomènes de variation en français moderne, entre autres la variable $\pm n e$, à la coexistence de deux grammaires (= variétés) du français ( $c f$. Coveney 2011, Dufter/Stark 2002, Gadet 2007 et Rowlett 2013 pour des réflexions critiques sur cette conception).

Ce groupe d'approches, que nous appelons l'hypothèse des deux grammaires, ${ }_{1}$, part de l'idée que les francophones ont accès à deux variétés ou grammaires du français, ce qui évite de postuler une seule

55 L'insertion d'un élément provoquerait la postulation de règles ultérieures pour définir la nature et les contextes de l'élément inséré, tandis que l'élimination d'un élément ne demanderait qu'une seule règle. 
grammaire qui permettrait une grande marge de variation difficile à modeler. En (85) figure une vue d'ensemble des variantes les plus importantes attribuées aux deux grammaires du français. Selon la conception de Massot (2010) et Zribi-Hertz (2011) ${ }^{56}$, les francophones possèdent deux grammaires (1 et 2 ) très similaires, mais qui se distinguent notamment par les variantes mentionnées en (85). Les locuteurs de français se trouveraient donc dans une situation de diglossie (cf. Ferguson 1959) comparable à celle qui existe entre l'arabe standard et les dialectes arabes ou entre l'allemand standard et les variétés suisse-alémaniques.

(85) Échantillon des variantes attribuées aux deux grammaires du français (cf. Koch/Oesterreicher ${ }^{2} 2011$ : 167-181, Massot 2010 : 201, Zribi-Hertz 2011 : $6-7)$

\begin{tabular}{|lll|}
\hline & Grammaire $1^{57}$ & Grammaire $2^{58}$ \\
\hline a. Négation & V-pas & ne-V-pas \\
b. Interrogation & il vient $?$ & vient-il ? \\
c. Pronoms & faut & fl faut \\
& {$[\mathrm{ja}]$} & Il y a \\
& {$[\mathrm{i}],[\mathrm{t}]$} & il, ty \\
& on & nous \\
& ça & cela \\
\hline
\end{tabular}

56 Koch/Oesterreicher (2011: 164ff, 181-182) n'adhèrent pas à la conception diglossique, mais soutiennent tout de même l'idée que 'le français parlé' forme une variété (donc une grammaire) de l'immédiat.

57 La grammaire 1 a été désignée dans la littérature par des termes divers : 'français (européen) colloquial' (cf. Culbertson 2010), 'français parlé' (cf. Koch/Oesterreicher $\left.{ }^{2} 2011\right)$, 'français démotique' ( $c f$. Massot 2010), 'français dialectal' (cf. ZribiHertz 2011), 'français informel', 'français avancé', 'français oral', 'français familier', 'français non-standard' etc. Certains traits de cette variété semblent coïncider avec une variété diastratique, décrite comme 'le français populaire'.

58 La grammaire 2 correspond au français standard, tel qu'il est prescrit par la norme ( $c f$. Grevisse/Goosse ${ }^{15} 2011$ ) et enseigné à l'école (aussi appelé 'français classique tardif' $c f$. Massot 2010, 'français formel', 'français correct' ou 'le bon usage').

$59 C f$. Koch/Oesterreicher ${ }^{2} 2011: 167$. 


\begin{tabular}{|c|c|c|}
\hline e. Relative ${ }^{60}$ & que relatif sujet & qui relatif sujet \\
\hline \multirow[t]{2}{*}{ f. Accord $^{61}$} & les lettres Pl.F que j’ai [екьi] & les lettres que j'ai [eksit] Pl.F \\
\hline & $c^{\prime}$ est $_{3 \mathrm{SG}}$ mes amis $3 \mathrm{Pl}$ & ce sont $3 \mathrm{Pl}$ mes amis $3 \mathrm{Pl}$ \\
\hline g. Redoublement du sujet & moi je viens, Pierre il vient & je viens, Pierre vient \\
\hline h. Phrases clivées & $\begin{array}{l}\text { c'est Xqui, } \\
\text { ce que/qui...c'est... }\end{array}$ & SVO \\
\hline \multirow[t]{3}{*}{ i. Temps et mode verbal ${ }^{62}$} & il a été & il fut \\
\hline & je vais aller & j'irai \\
\hline & je voulais qu'il vienne & je voulais qu'il vînt \\
\hline j. article indéfini pluriel ${ }^{63}$ & des bons vins & de bons vins \\
\hline k. liaison 'facultative' & 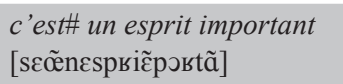 & 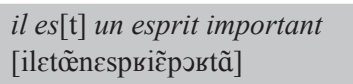 \\
\hline
\end{tabular}

Zribi-Hertz (2011 : 3) cite à cet égard la définition de diglossie de Crystal ( $\left.{ }^{3} 1991: 104\right)$ :

(86) Définition de diglossie

Diglossia (diglossic). A term used in sociolinguistics to refer to a situation where two very different varieties of a language co-occur throughout a speech community, each with a distinct range of social function. Both varieties are standardised to some degree, are felt to be alternatives by native-speakers and usually have special names. Sociolinguists usually talk in terms of a high $(\mathrm{H})$ variety and a low $(\mathrm{L})$ variety, corresponding broadly to a difference in formality: the high variety is learned in school, tends to be used in church, on radio programmes, in serious literature, etc., and as a consequence it has greater social prestige; the low variety in family conversations, and other relatively informal settings. Diglossic situations may be found in Greek (High: Katharevousa; Low: Dhimotiki), Arabic (High: classical; Low : colloquial), and some varieties of German (H: Hochdeutsch; L: Schweizerdeutsch, in Switzerland). (Crystal ${ }^{3} 1991$ : 104)

Dans cette optique, la grammaire 1 en (85) correspond à la variété basse (L), acquise inconsciemment en famille à l'âge préscolaire et de façon

60 Cf. Koch/Oesterreicher ${ }^{2} 2011: 167$.

$61 C f$. Koch/Oesterreicher ${ }^{2} 2011: 169$.

$62 C f$. Koch/Oesterreicher ${ }^{2} 2011: 171$.

$63 C f$. Koch/Oesterreicher ${ }^{2} 2011: 169$. 
active grâce au contact avec les interlocuteurs intimes dans toutes les situations sans caractère protocolaire ${ }^{64}$, par exemple les conversations ou le sport.

La variété prestigieuse $(\mathrm{H})$, par contre, est enseignée et apprise à l'école et se trouve sous l'influence du médium graphique et de la tradition littéraire. Elle est activée dans toutes les situations de protocole (dans le contact avec les institutions officielles, à l'église etc.) et se caractérise, contrairement à la grammaire 1, par une compétence variablement prononcée en fonction du degré de scolarisation des locuteurs. Tandis que tous les francophones possèdent une compétence passive dans la grammaire 2, leur production langagière générée par cette grammaire est caractérisée par des zones d'insécurité et d'hypercorrection:

(87) Insécurité et hypercorrection en grammaire 2 (cf. Rowlett 2013)

\begin{tabular}{|lll|}
\hline & Norme & Insécurité \\
\hline SUBJ & Le fait que tu dises... & Après que tu dises... ${ }^{65}$ \\
\hline IND & Après que tu dis... & Le fait que tu dis... \\
\hline
\end{tabular}

Zribi-Hertz (2011 : 12) soutient qu'il serait théoriquement et didactiquement préférable de décrire la compétence des francophones en termes d'une diglossie entre les grammaires 1 et 2 comprenant une zone d'intersection. Cette zone contiendrait un échantillon de variantes neutres (donc ni $\mathrm{H}$ ni L) qui pourraient être produites par les deux grammaires, ce qui provoquerait chez les locuteurs l'illusion de parler 'une seule langue', à savoir 'le français'. Le modèle diglossique des trois zones selon Zribi-Hertz (2011: 12) (auquel adhère également Massot 2010) figure en (88). La zone 1 correspond à notre grammaire 1, la zone 2 correspond à l'intersection entre les deux grammaires et la zone 3 à notre grammaire 2 .

64 Zribi-Hertz (2011: 3) précise que l'adjectif «(in)formel est un faux ami adapté de l'anglais (in)formal, qui implique une notion de protocole: a formal dinner est un dîner protocolaire».

65 Formé de façon analogue à avant que+SUBJ. 
(88) L'hypothèse de la diglossie francophone selon Zribi-Hertz (2011: 12)

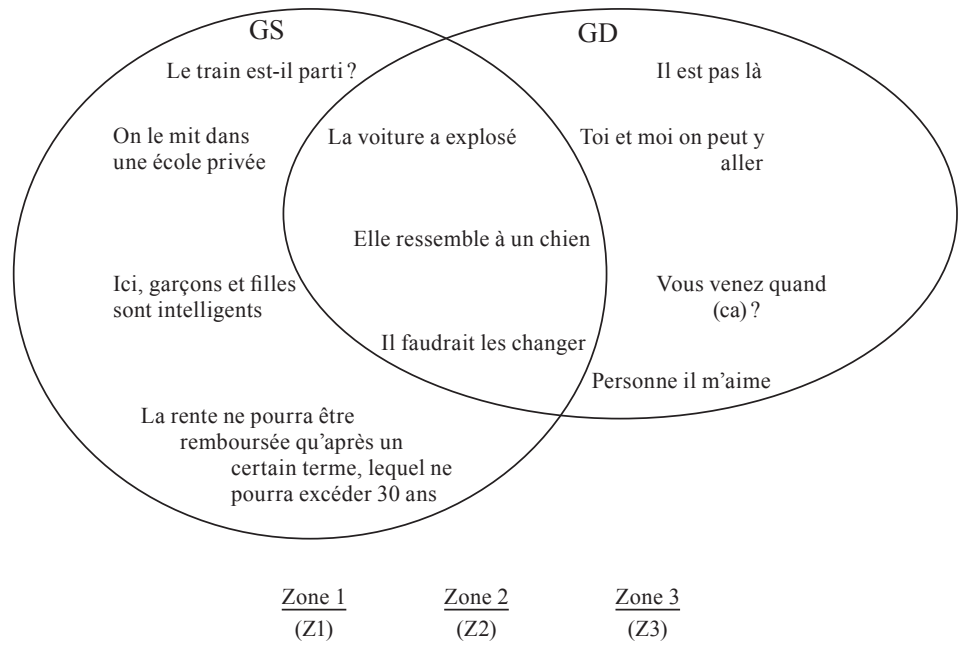

Massot (2010: 98) soutient que les francophones ne mélangent pas les variantes des grammaires 1 et 2 au niveau d'un même énoncé, bien qu'ils alternent continuellement les deux grammaires. Pour la distribution du $\pm n e$ dans notre corpus, ceci signifie que la variante -ne devrait s'associer davantage aux variantes de la grammaire 1, tandis que +ne devrait se trouver en cooccurrence avec les variantes de la grammaire 2 .

Une conception similaire, que nous appelons l'hypothèse des deux grammaires $_{2}$, a été récemment défendue par Culbertson (2010). La particularité de son approche réside dans la combinaison de l'hypothèse de l'incompatibilité entre ne et les clitiques sujet (présentée dans la section précédente) avec celle d'une diglossie en France. Son travail s'inscrit dans une série d'ouvrages (cf. Auger 1994, Kaiser 2008, Miller/Sag 1997, Roberge 1990, Zribi-Hertz 1994) qui soutiennent l'idée que les pronoms clitiques du français seraient réanalysés en tant que marques d'accord préfixées au verbe. Culbertson (2010: 96) soutient que si les pronoms clitiques sont devenus des affixes verbaux grammaticalisés sans statut argumental, le clitique ne (un élément non affixal) ne pourrait pas intervenir entre le préfixe et sa base: 
(89) On a lexicalist view of morphology, if ne is not part of the verbal inflectional material, then although it may freely intervene between lexical subjects and the verb, it may not do so between the verb and the subject-clitic prefix. (Culbertson 2010: 96) ${ }^{66}$

Culbertson (2010) adhère à l'hypothèse selon laquelle les clitiques sujet sont des marques d'accord affixées au verbe, mais uniquement dans le français de l'immédiat, c'est-à-dire dans la grammaire $1^{67}$, comme la structure en (90) le montre.

(90) Grammaire 1: les clitiques sujet sont des marques d'accord préfixées au verbe ( $c f$. par exemple Jaeggli 1982, Auger 1994

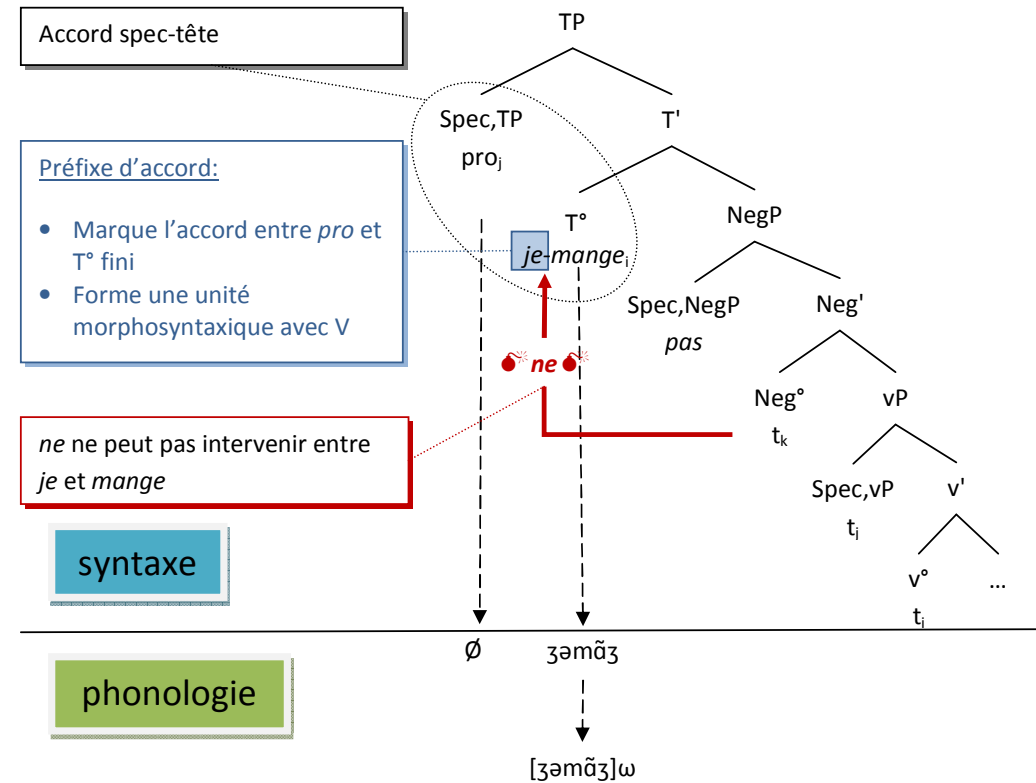

66 Dans une approche lexicaliste, le processus de flexion est présyntaxique, ceci signifie que les formes lexicales insérées en syntaxe comprennent tous les affixes. $C f$. Culbertson (2010: 96) pour une exploration de la même hypothèse dans le cadre d'une approche non-lexicaliste.

67 Dans la terminologie de Culbertson (2010: 85), notre grammaire 1 est appelée 'français européen colloquial' («European colloquial French»). 
Dans la grammaire 1, je est un préfixe qui marque l'accord entre Spec, TP et T (cf. Culbertson 2010). Ceci implique que je n'est pas un argument verbal et qu'il ne reçoit ni de cas ni de rôle thématique. Par contre, il marque morphologiquement les caractéristiques $\Phi$ (personne et nombre) du sujet au verbe fini.

Une conséquence de cette hypothèse (mentionnée mais non explorée par Culbertson 2010: 124) est que la grammaire 1 est analysée comme une langue pro-drop (ou à sujet nul) ${ }^{68}$. Ceci signifie que, dans les phrases sans sujet lexical, la position Spec,TP est occupée par un pronom sujet phonologiquement vide, spécifié en termes de personne et nombre, et capable d'assumer un cas et un rôle thématique $(=p r o)$. Le préfixe d'accord je et le verbe fini forment alors une seule unité morphosyntaxique cohérente à l'intérieur de laquelle aucun élément syntaxique (mais seulement d'autres affixes) ne peut intervenir. Ne étant un clitique (c'est à dire une unité syntaxique), il ne peut donc pas intervenir entre je et mange.

En ce qui concerne le français standard (= grammaire 2), Culbertson (2010) défend, par contre, l'analyse 'classique' des sujets clitiques du français ( $c f$. Belletti 1999, De Cat 2007, Kayne 1975, 1991 Rizzi 1986). Ceux-ci ne seraient joints au verbe que dans le composant phonologique (= clitiques phonologiques). Ils occupent en syntaxe la position canonique du sujet $\mathrm{Spec}, \mathrm{TP}$ et reçoivent un rôle thématique et un cas, tout comme les sujets lexicaux.

68 Selons l'hypothèse des deux grammaires 2 , la grammaire 1 est une langue à sujet nul qui diffère typologiquement du français standard (= grammaire 2). Cette affirmation va donc beaucoup plus loin que celle de l'hypothèse des deux grammaires $s_{1}$, qui soutient que la différence entre les deux grammaires réside dans certaines formes et constructions, présentées en (85). 
(91) Grammaire 2: les clitiques sujet sont des arguments syntaxiques (cf. par exemple Kayne 1975, 1991, Rizzi 1986, Belletti 1999, De Cat 2007)

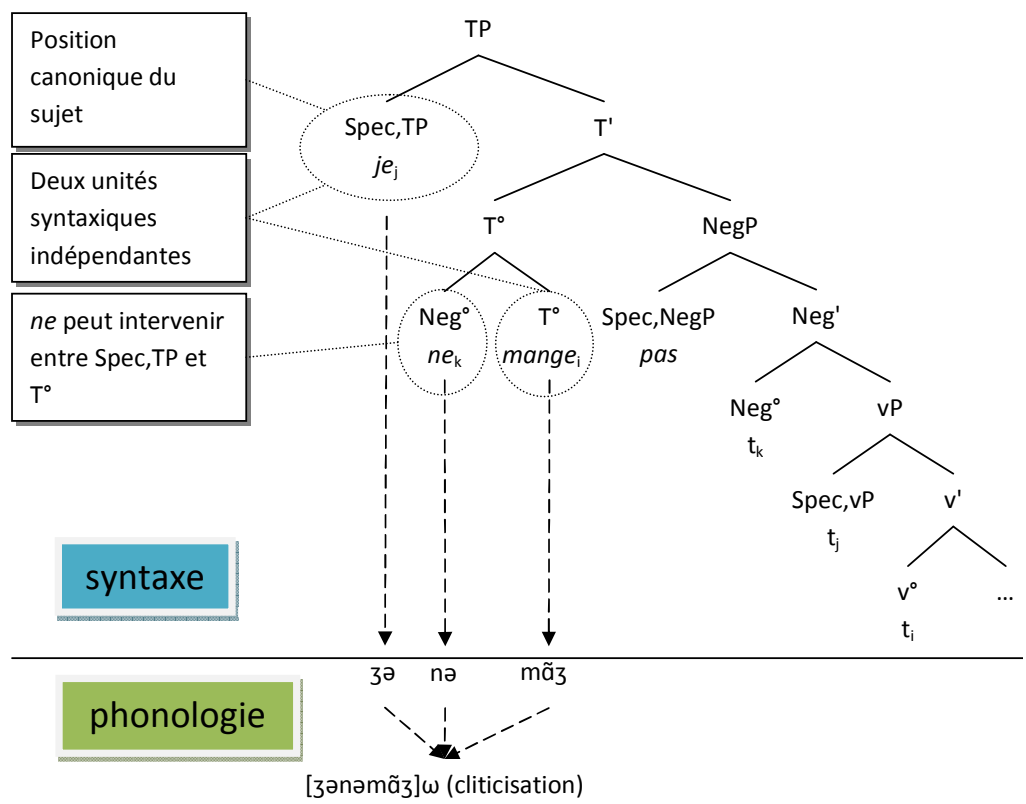

En suivant cette hypothèse, dans la grammaire $2, j e, t u$, $i l$ etc. sont des clitiques phonologiques, syntaxiquement équivalents aux sujets lexicaux, qui reçoivent un cas et un rôle thématique et qui sont situés ${ }^{69}$ dans la position canonique des sujets Spec,TP. Les éléments je et mange en (91) sont donc deux unités séparées entre lesquelles d'autres éléments syntaxiques, tels que le clitique ne, peuvent intervenir. Selon l'analyse en termes de NegP ( $c f$. section 2.1.3.1), ne est élevé à T par un mouvement tête-à-tête. Au niveau phonologique, c'est-à-dire postyntaxiquement, je est cliticisé sur le verbe ou, comme en (91), sur un autre clitique, et le verbe et le(s) clitique(s) forment alors une unité phonologique.

69 Plus précisément, les sujets sont déplacés à Spec,TP depuis leur génération en Spec,VP (cf. VP-internal subject hypothesis (VISH)) 
Toutefois, cette analyse s'avère incapable d'expliquer les asymétries syntaxiques et phonologiques entre différents types de sujet, qui ont été vérifiées empiriquement quant à la réalisation de ne ( $c f$. section 2.2.5.1). Ceci constitue l'un des problèmes majeurs du parallélisme entre les sujets lexicaux et les clitiques, revendiqué par l'analyse en (91).

Cette problématique est résolue par Culbertson (2010) en ayant recours à l'alternance codique entre les deux grammaires dans le sens de l'hypothèse des deux grammaires s $_{1}$. La présence variable de ne dans les corpus s'explique, selon Culbertson (2010), par l'alternance entre les grammaires 1 et 2. Culbertson (2010: 98) modélise l'alternance codique à l'aide de deux traits binaires: les clitiques sujet peuvent être + ou - élevés, c'est-à-dire situés en Spec,TP ou non, et la particule ne peut être + ou - réalisée. Le clitique +élevé, celui du français standard (= grammaire 2), est analysé comme un DP lexical et se trouve dans la position Spec,TP, tandis que le clitique -élevé, celui de la grammaire 1 , est un affixe en TP. La réalisation de ne, qui intervient entre le sujet et le verbe, est possible avec un clitique +élevé de la grammaire 2, mais impossible avec un clitique -élevé de la grammaire 1, car aucun matériel lexical (sauf d'autres affixes) ne peut intervenir entre un affixe et sa base. Culbertson (2010) évalue les capacités explicatives d'un modèle monogrammatical quant à la réalisation variable de ne contre celles d'un modèle d'alternance codique, $c f$. (92). Le modèle monogrammatical contient uniquement la grammaire 2, c'est-à-dire que tous les clitiques sujet sont analysés comme des clitiques phonologiques situés en Spec,TP (comme proposé par De Cat 2007 et Rizzi 1986, entre autres). Dans le modèle d'alternance codique, deux possibilités d'analyse des clitiques existent: premièrement, ils peuvent être analysés comme des affixes en $\mathrm{T}^{\circ}$ (générés par la grammaire 1) ou deuxièmement comme des arguments en Spec,TP (générés par la grammaire 2). 
(92) L'alternance entre deux grammaires selon Culbertson (2010: 98, simplifiée ${ }^{70}$ )

\begin{tabular}{|l|lll|l|ll|c|}
\hline modèle & grammaire & sujet & Spec,TP & ne & sujet & Spec,TP & $n e$ \\
\hline une seule grammaire & 2 & DP & + & + & clitique & + & + \\
\hline $\begin{array}{l}\text { alternance codique entre } \\
\text { deux grammaires }\end{array}$ & 1 & DP & + & + & clitique & - & - \\
& 2 & DP & + & + & clitique & + & + \\
\hline
\end{tabular}

Le modèle monogrammatical prédit une réalisation constante de ne, car aussi bien le sujet DP que le clitique sujet se trouvent en Spec,TP, et la réalisation de ne en position préverbale reste intacte. Tandis que les sujets DP ont toujours un statut argumental, indépendamment de la grammaire sous-jacente, le statut des clitiques peut varier dans le modèle d'alternance codique, ce qui interfère avec la réalisation de ne.

Le modèle mixte prédit une présence variable de ne, car deux analyses alternatives des clitiques sujet coexistent: les clitiques affixaux générés par la grammaire 1 bloquent la réalisation de $n e$, tandis que les clitiques argumentaux générés par la grammaire 2 la permettent. Les fréquences de ne attestées empiriquement, qui sont généralement basses mais varient en fonction du corpus ( $c f$. section 2.2), seraient donc mieux expliquées en termes d'un modèle mixte que d'un modèle monogrammatical. Dans les corpus, ne apparaît en grande partie dans le contexte de sujets lexicaux (qui n'interfèrent jamais avec la réalisation de la particule), et le résidu de ne avec les sujets clitiques découlerait de la grammaire 2, qui génère des clitiques qui se comportent parallèlement aux sujets DP. Selon Culbertson (2010), le modèle d'alternance codique serait donc plus apte à expliquer la variation de \pm ne qu'un modèle qui n'assume qu'une seule grammaire invariable.

Cependant, l'analyse de Culbertson (2010) pose une série de problèmes théoriques et empiriques qu'il faut noter. Tout d'abord, dans l'op-

70 Les combinaisons de traits impossibles ou inclassables ont été supprimées. Culbertson (2010: 98) assume ultérieurement l'existence de sujets DP en absence de ne et de sujets clitiques en spéc,TP en absence de $n e$, mais il reste peu clair à partir de quelle grammaire ces combinaisons sont générées ou s'il s'agit d'interférences entre les deux grammaires. 
tique de Culbertson (2010), toute variation constatée empiriquement, comme celle de $\pm n e$, est expliquée uniquement en termes d'alternance codique. Ceci impliquerait, au niveau théorique, qu'aucune variation interne au système d'une grammaire n'est admise. Au niveau empirique, cette approche prédit que nous devrions pouvoir observer l'alternance codique effectuée par les locuteurs dans les corpus. Autrement dit, dans les corpus, les variantes attribuées à l'une des deux grammaires devraient se regrouper selon les situations de communication et les passages d'une grammaire à l'autre devraient être reconnaissables par un changement des variantes linguistiques employées. Comme le soutient également Massot (2010), les variantes attribuées aux grammaires 1 ou 2 ne devraient pas être en cooccurrence au niveau du même énoncé. Nous discuterons en section 5.1.3 si ces conditions sont remplies dans notre corpus et si l'approche diglossique semble fondée empiriquement.

\subsubsection{L'approche pragmatique: ne comme une particule emphatique}

Pour le français hors de France, une explication pragmatique de la variation du ne a été envisagée depuis les analyses de Sankoff/Vincent (1980). Récemment, elle a gagné un nouvel intérêt et un fondement théorique grâce aux travaux de van Compernolle (2008a,b), FonsecaGreber (2007) et Poplack/St-Amand (2009).

Sankoff/Vincent (1980) et Poplack/St-Amand (2009) rapportent que les rares instances de ne (environ $1 \%+n e$ ) dans leurs corpus de français québécois apparaissent précisément dans des contextes sérieux ou moralisateurs concernant la religion, comme en (93)a, le bon usage, comme montré en (93)b, ou l'éducation, $c f$. (93)c.

(93) +ne-pragmatique dans des contextes 'sérieux' en québécois (cf. Poplack/St-Amand 2009: 535)

a. Le Bon Dieu a dit «tu ne tueras point»

b. On a vieilli en faisant le choix de bien parler notre français ou de ne pas bien le parler

c. Puis si tu parlais, il te donnait un copiage, il te donnait deux feuilles: "je ne dois pas parler en classe» 
Par contre, les facteurs extralinguistiques (le statut socio-économique ou éducatif des locuteurs) et linguistiques (le sujet, le contexte phonologique etc.) cités dans la littérature (socio)linguistique concernant ce sujet n'auraient pas d'influence particulière dans le corpus de Poplack/ St-Amand (2009: 535). Nous tenons à rappeler que le corpus de Poplack/ St-Amand (2009) a été enregistré entre 1940 et 1960 avec des locuteurs très âgés et qu'il représente donc l'état le plus ancien de français phonique disponible (cf. section 2.2.2). Au Québec, il semble donc que le $n e$ est conservé depuis presque cent ans dans certains contextes rares et pragmatiquement définis.

Mais l'hypothèse du ne-pragmatique s'avère-t-elle également valable pour le français européen? Les études qualitatives de van Compernolle (2008b) sur un corpus de chat francophone géographiquement non-spécifié et de van Compernolle (2009) et de Fonseca-Greber (2007) sur des corpus phoniques issus de France et de Suisse révèlent que les francophones hors du Canada effectuent apparemment, eux aussi, des micro-alternances stylistiques (qui ont une influence sur la variable $\pm n e$ ) en fonction de facteurs 'pragmatiques' éventuellement comparables à ceux attestés en français québécois:
(94) Les contextes 'pragmatiques' de $+n e$
a. L'autopromotion du locuteur
b. Le jeu avec la langue
c. Le discours rapporté
d. La discussion des sujets 'sérieux'
e. L'attitude personnelle du locuteur envers le sujet de communication
f. Le caractère emphatique ou contrastif de la négation

La liste en (94) pourrait être élargie en suivant (au moins) deux pistes.

Premièrement, les contextes en (94)a-d (éventuellement aussi e) pourraient être explicables à l'aide de l'audience design ${ }^{71}$ (cf. Bell 1984, 2001).

71 Bell $(1984,2001)$ soutient que le dialogue est l'instance naturelle de la langue et que le comportement linguistique des locuteurs (c'est-à-dire, les émetteurs du message) n'est évaluable qu'en considération du public (donc des destinataires). Dans l'approche de l'audience design, le style est défini comme le comportement linguistique d'un locuteur envers un public ( $c f$. Bell 2001: 141-142). 
Dans la conception de Bell (2001: 154), la variation intralocuteur dérive de et reflète la variation interlocuteur. L'alternance entre différents styles en fonction d'un thème, d'un sujet parlant ou d'une situation dérive de l'association sous-jacente des sujets, des thèmes et des situations avec les interlocuteurs prototypiques. Ceci signifie que les particularités linguistiques peuvent servir comme ressource d'identification avec un groupe. Analysée selon l'approche de Bell $(1984,2001)$, la variante $\pm n e$ serait donc un moyen d'autopromotion des locuteurs en fonction d'un public potentiel.

Deuxièmement, les contextes de + ne en (94)f (éventuellement aussi e et d) ressemblent aux contextes de réalisation de la particule discursive ou emphatique en du flamand (cf. Breitbarth/Haegeman 2010, à paraître). Contrairement à la majorité des langues germaniques, qui ont atteint l'étape finale du cycle de Jespersen, les dialectes flamands ont conservé, bien que de façon facultative, une particule préverbale de négation. À première vue, ces dialectes semblent donc, comme le français, demeurer dans la quatrième étape du cycle négatif, notamment celle de la variation entre la négation bi- et monopartite ( $c f$. section 2.1.2.1).

Toutefois, dans certaines variétés de flamand, l'optionalité de en dans des contextes négatifs n'est qu'apparente ( $c f$. Breitbarth/Haegeman 2010: 62). En effet, en apparaît en plus du négateur standard nie( $(t)$ et d'autres éléments négatifs précisément dans les négations emphatiques qui refusent une expectation sous-jacente ou explicite dans le discours:

Il dérive sa signification de l'association des particularités linguistiques avec certains groupes sociaux. Bell (2001 : 143) soutient que les locuteurs conçoivent leur style avant tout pour et en réaction à un public. L'audience design est applicable à tout code ou niveau d'un répertoire linguistique, aussi bien monolingue que plurilingue, l'approche serait donc compatible avec les hypothèses diglossiques du français ( $c f$. section 2.3.3). Bell (2001 : 146) propose aussi, en plus de la dimension 'réactive' de l'audience design, une dimension 'active', dans laquelle une alternance de styles peut initier un changement de la situation (au lieu d'en résulter). Ces alternances stylistiques 'actives' sont désignées comme referee design. 
(95) Les contextes de en-emphatique en flamand de l'ouest (cité d'après Breitbarth/Haegeman 2010: 65)
a.
A: Geefme nen keer
Valère zenen
telefoon.
give me once
Valère his
phone number
'Can you give me Valère's phone number?'
b. B: K'(en) een-k ik Valère zenen telefon nie.
I (en) have -I I Valère his phone NEG
'I don't have Valère's number.'
c. B: K-zeggen jen toch dan-k em nie (en)-een.
I say you particle that -I him NEG (en) have
'I am telling you I don't have it.' （Lapscheure, Haegeman 2002: 11)

(96) Les contextes de en-emphatique en flamand de l'est

(cf. Breitbarth/Haegeman 2010: 66)

a. Ge weunt al vijftien jaor in Gent, in g'en ken nog d'Universiteit nie? you live already fifteen years in Ghent and you en know still the university not

'What? You have lived in Ghent for fifteen years, and you are telling me you still don't know the university?!'

b. Dat en is toch mijn schuld nie!

that $e n$ is PRT my fault not

'You can't say that this is MY fault!'

(Ghent, Overdiep 1937: 456-457)

Les contextes de refus permettent l'ajout de la particule en au négateur standard. L'exigence, voire l'expectation formulée en (95)a, est déclinée en (95)b et même rejetée énergiquement en (95)c. L'exemple en (96) a fait allusion à l'université très connue de Ghent, la présupposition, selon laquelle tous les habitants de Ghent connaissent l'université, est donc implicitement donnée et le fait qu'un habitant de cette ville puisse l'ignorer provoque de l'étonnement et de l'incrédulité, ce qui est souligné par l'emploi de la particule en. En (96)b, une accusation implicite ou explicite préalable est refusée et l'emploi de en indique le caractère emphatique de l'énoncé.

Breitbarth/Haegeman (2010) concluent que c'est un changement linguistique dans les dialectes flamands qui a 'sauvé' en de l'extinction. En ne serait plus une particule de négation, mais une particule d'emphase ou de discours ( $c f$. Breitbath/Haegeman à paraître). Ceci signifie que les dialectes flamands auraient également atteint l'étape finale du cycle de Jespersen et que la quantité faible mais stable de en dans le dis- 
cours constituerait une innovation en dehors du cycle négatif, comme l'illustration en (97) le montre.

(97) La particule pragmatique: une issue alternative au cycle de Jespersen?

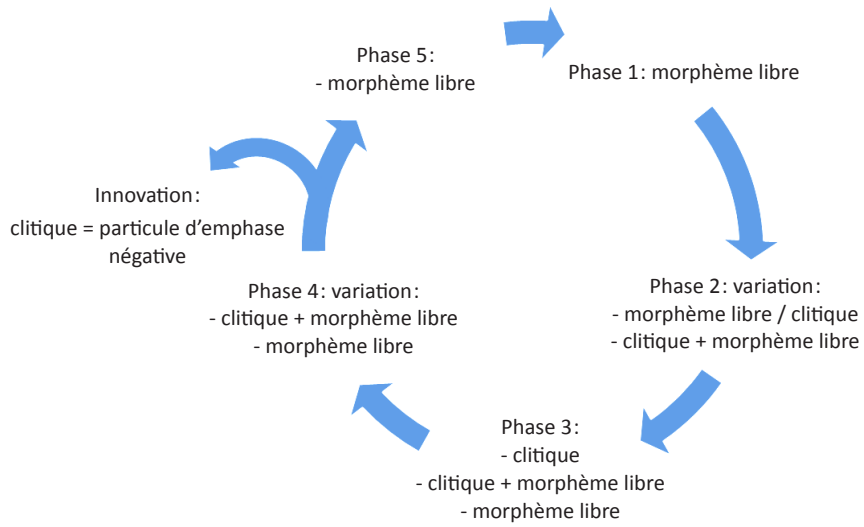

L'on peut se demander si une telle issue alternative au cycle de Jespersen serait également imaginable pour le français. Nous aborderons cette question en section 5.1.5 à la lumière des résultats de notre analyse de corpus.

\subsection{Résumé du chapitre 2}

Ce chapitre forme la base théorique et empirique de l'objectif de cette thèse, à savoir l'analyse de la réalisation variable de l'expression de la négation de phrase en français contemporain.

Le sous-chapitre 2.1 fournit les bases logiques, terminologiques et typologiques de l'expression de la négation dans les langues naturelles et décrit l'évolution de la négation en français ainsi que son expression en français moderne. Nous avons introduit une classification tripartite de la concordance négative selon Haspelmath (1997: 201), qui distingue la concordance négative (français standard: ne personne: $\neg p=\neg p$ ) 
de celle partielle, où l'interprétation dépend de la position syntaxique de l'indéfini négatif (par exemple en italien) et de la non-concordance (cf. angl. standard: not nobody $=$ somebody, $\neg \neg p=p$ ).

En français, la négation de phrase est issue du latin non/ne et est renforcée, d'abord facultativement, par des éléments nominaux comme pas, personne, rien etc., qui deviennent par la suite des éléments négatifs (cf. cycle de Jespersen: non/ne $\mathrm{V} \rightarrow$ ne $\mathrm{V}$ pas $\rightarrow \mathrm{V}$ pas, Jespersen 1917, 1924). Le début de l'omission de ne dans les phrases négatives, observable en français moderne, reste débattu. Même si des chercheurs renommés comme Ashby (1981) et Martineau/Mougeon (2003) soutiennent qu'il s'agit d'un changement récent et rapide ayant débuté au $19^{\mathrm{e}}$ siècle, la position alternative de Dufter/Stark (2007) et Martineau $(2009,2011)$ se renforce actuellement. Selon celle-ci, l'absence de $n e$ serait plus ancienne (vers le $17^{\text {e }}$ siècle ou avant) et serait éventuellement mieux analysée en termes d'une variation (relativement stable) jusqu'à nos jours.

En français contemporain, la négation de phrase est exprimée par la particule négative pas en coprésence variable avec la particule proclitique $n e$. Au niveau syntaxique, nous avons adapté, là où une modélisation syntaxique est nécessaire, le modèle du syntagme négatif NegP (selon Rowlett 1998 basé sur Pollock 1989), en admettant que, dans les cas où elle est absente, la particule ne est effacée phonologiquement. L'analyse de corpus portera sur l'absence et présence du ne avec les éléments négatifs, comme personne, rien, nul $\mathrm{N}$, jamais, plus, ni...ni etc. et aussi avec le terme restrictif que, même si ce dernier n'exprime pas la négation. Par contre, les éléments de polarité négative, comme quoi que ce soit et rien et personne, employés comme termes de libre choix dans les contextes hypothétiques et interrogatifs n'expriment aucune négation et seront exclus de l'analyse de corpus.

Dans le sous-chapitre 2.2, nous avons présenté les influences potentielles sur $\pm n e$, en les structurant selon la nature du corpus (graphique ou phonique) et des facteurs d'influence (extra vs. intralinguistique), qui semblent être les mêmes dans les deux types de corpus (en partie déjà depuis le $17^{\mathrm{e}}$ siècle).

Au niveau extralinguistique, plus précisément d'un point de vue diatopique, le ne semble plus stable au sud de la France, tandis qu'il a 
presque disparu au Canada. Quant à l'âge des locuteurs, la particule ne est généralement plus répandue chez les adultes que chez les jeunes et ce sont les premiers qui montrent également une variation plus articulée en fonction de la situation de communication. Toutefois, l'influence de ce dernier facteur extralinguistique n'a pas encore été suffisamment étudiée. La variation intrapersonnelle et surtout son interdépendance avec les facteurs linguistiques constitueront donc l'un des centres d'intérêt de ce travail.

Au niveau linguistique, l'influence la plus puissante est le type de sujet grammatical. Ce sont surtout certains clitiques légers et fréquents (je, $t u, c e$ ) qui provoquent l'absence de $n e$, tandis que les sujets lexicaux et les pronoms nous et vous manifestent des taux de ne relativement élevés. À cet égard, nous avons présenté une classification des types de sujet en tant que continuum ( $c f$. aussi Bossong 1998), qui sera pertinente pour l'explication prosodique de la variation \pm ne proposée dans le chapitre 5 de ce travail.

Contrairement à l'influence du type de sujet, l'influence des proclitiques non-sujet reste diffuse. Les éléments négatifs non-clitiques et les temps verbaux provoquent peu de variation $\pm n e$, leur influence serait éventuellement mieux analysée en tant qu'épiphénomène de la fréquence et du contexte phonologique. Par contre, il semble clair que la réalisation de ne est plus élevée dans les subordonnées que dans les principales. Au niveau de la phonologie segmentale, la réalisation de ne est inévitablement liée à la réalisation du schwa, qui forme le noyau vocalique des clitiques je, ce, me, le et de ne même et dont l'absence dans la chaîne clitique conduit à des séquences consonantiques imprononçables. Au niveau de l'articulation, un haut débit de parole implique une grande absence de ne. Le rôle de la phonologie suprasegmentale n'a pas encore été exploité complétement et sera discuté à plusieurs reprises lors du présent travail.

L'ensemble des travaux empiriques concernant \pm ne a été fortement critiqué par Gadet (2000), qui revendique, entre autres, une plus grande authenticité des données et une meilleure interprétation des résultats statistiques. Nous essayerons de combler certaines des lacunes mentionnées par Gadet (2000) lors de notre analyse. 
Finalement, le sous-chapitre 2.3 présente quatre hypothèses principales qui ont été formulées afin d'expliquer la variation $\pm n e$ en français moderne.

L'explication la plus ancienne est celle des sociolinguistes qui affirment que $\pm n e$ indiquerait la classe sociale (ou l'âge) du locuteur. Cependant, et en tenant compte de la complexité des sociétés modernes et de nombreuses autres influences sur la variable, les analyses de corpus ont du mal à appuyer cette thèse.

La deuxième hypothèse se base sur les résultats empiriques qui confirment le fait que les sujets lexicaux incitent à la réalisation de $n e$, tandis que certains clitiques, comme $j e, t u$ et $c e$ semblent l'inhiber. Toutefois, la modélisation syntaxique de cette tendance s'avère pénible, car d'une part les corpus contiennent des contre-exemples à la généralisation (sujet DP $\rightarrow+n e$ et sujet clitique $\rightarrow-n e$ ), et d'autre part, car les sujets DP et les clitiques ne forment pas de dichotomie, mais plutôt un continuum entre des éléments légers et lourds.

La troisième hypothèse, celle de l'approche diglossique, est née de la linguistique variationnelle et est également soutenue depuis quelque temps par des syntacticiens. Les avocats de l'hypothèse des deux grammaires $_{1}$ affirment que les francophones seraient en principe bilingues et qu'ils maitriseraient deux grammaires: une grammaire 1, acquise de manière naturelle des parents et utilisée dans tous les contextes privés, et une grammaire 2, apprise de manière éventuellement imparfaite à l'école et par la tradition littéraire (littérature, journaux, bureaucratie etc.). Toute variation observable en français moderne, non seulement celle de $\pm n e$, mais également la forme des pronoms personnels, le système temporel et modal etc. serait explicable à travers l'alternance codique entre les deux grammaires, effectuée inconsciemment par tous les locuteurs du français. L'hypothèse des deux grammaires 2 combine l'idée de l'alternance codique avec celle de l'influence du type de sujet: dans la grammaire 1, les pronoms clitiques seraient désormais des marques d'accord préfixées au verbe qui ne permettent aucune interférence du clitique $n e$.

La quatrième hypothèse explique les contextes dans lesquels ne est présent en fonction de critères pragmatiques. Dans un cadre d'analyse 
stylistique, les occurrences de \pm ne pourraient être analysées comme des ressources d'autopromotion du locuteur en fonction de l'adaptation à un publique envisagé ( $c f$. Bell 1984, 2001). En plus de cela, le résidu de $n e$ (en français canadien stable à environ $1 \%$ the depuis plus de cent ans) pourrait être interprété comme une issue alternative du cycle de Jespersen, comme la voie qu'ont apparemment prise les particules clitiques négatives en flamand qui sont devenues des particules d'emphase.

La validité de ces quatre hypothèses explicatives de $\pm n e$ face à nos données de corpus sera discutée dans le sous-chapitre 5.1. 
Charlotte Meisner - 978-3-0343-2332-1

Downloaded from PubFactory at 01/11/2019 02:38:59AM

via free access 


\section{Méthodologie: le corpus}

\subsection{Comment accéder au corpus?}

Le corpus à la base de cette étude et le manuel d'utilisation sont disponibles en ligne sous : <http://server.linguistik.uzh.ch/cmeisner2013-tzero> login: «TZ-Reader»

mot de passe «TZero@ling!»

\subsection{Le corpus: motivation, conception et possibilités de requête ${ }^{72}$}

La base de données T-zéro est née de la nécessité de créer un corpus qui permette une analyse détaillée de la variable $\pm n e$, c'est-à-dire un corpus qui permette l'étude de l'axe intrapersonnel de la variation linguistique et qui soit transcrit phonétiquement afin de relever les réalisations concrètes des formes linguistiques. Ces deux critères sont indispensables pour l'analyse des facteurs extralinguistiques (par exemple un locuteur dans différentes situations de communication) et linguistiques (par exemple la réalisation des clitiques sujet) qui déterminent la variable $\pm n e$. De plus, il était souhaitable que notre corpus contienne également des données de Suisse romande. Avant la création de T-zéro, aucun corpus ne correspondait à nos besoins, et cela malgré la présence considérable de moyens et de grands corpus du français oral, leur croissante disponibilité en ligne et l'évolution des systèmes de transcription.

72 Je remercie toutes les personnes qui ont contribué à la création de ce corpus, avant tout les participants, et en particulier Aurélia Robert-Tissot et David Paul Gerards, qui m’ont soutenue dans la transcription des données. 
(98) Comparaison d'une sélection de corpus du français oral

\begin{tabular}{|c|c|c|c|c|c|}
\hline Corpus & $\begin{array}{l}\text { Accès } \\
\text { en } \\
\text { ligne }\end{array}$ & Diaphasie $^{73}$ & $\begin{array}{l}\text { Suisse } \\
\text { romande }\end{array}$ & Intrapersonnel $^{74}$ & $\begin{array}{l}\text { Transcription } \\
\text { API }\end{array}$ \\
\hline Ludwig 1988 152p & - & + & - & - & - \\
\hline $\begin{array}{l}\text { C-ORAL-ROM } 2005 \\
\text { (440.000 mots) }\end{array}$ & - & + & - & - & - \\
\hline CIEL_F 2008-2010 & + & + & - & - & - \\
\hline $\begin{array}{l}\text { PFC 2004- } \\
(1.400 .000 \text { mots })\end{array}$ & + & + & Genève/Nyon & - & - \\
\hline $\begin{array}{l}\text { T-zéro } 2008-9 \\
\text { (16.150 mots) }\end{array}$ & + & + & Neuchâtel & + & + \\
\hline
\end{tabular}

Dans le tableau (98), nous présentons une brève sélection des corpus oraux du français (pour un panorama des corpus existants $c f$. Bilger 2002, Cappeau/Gadet 2007, Pusch 2002, 2007, Pusch/Kabatek 2005 éd., Pusch/Kabatek 2005). Nous citons, tout d'abord, l'ouvrage de Ludwig (1988) en tant que représentant d'une multitude d'excellents corpus récoltés, transcrits et décrits par un auteur ou une petite équipe de recherche, et publiés sous forme de livre ( $c f$. également BlancheBenveniste et al. éd. 2002). Les trois corpus suivants, C-ORAL-ROM (cf. Cresti/Moneglia 2005), CIEL_F ${ }^{75}$ et $\mathrm{PFC}^{76}$ (cf. Durand et al. 2002, 2005, 2009), sont de larges projets de recherche internationaux, financés par divers fonds nationaux, européens ou privés et effectués par des équipes régionales qui s'accordent sur des standards méthodologiques communs. Ces projets permettent la documentation d'une quantité considérable de données linguistiques du français oral à travers les continents (le PFC comporte plus de 70 points d'enquête) et les situations de communication (CIEL_F vise à documenter une multitude de situations naturelles). Malgré les avantages évidents des corpus exis-

\footnotetext{
732 locuteurs/ 2 situations.

741 locuteur/2 situations.

75 Corpus International Ecologique de la Langue Française (<http://www.ciel-f. $\operatorname{org} />)$.

76 Projet Phonologie du Français Contemporain (<http://www.projet-pfc.net/>).
} 
tants, ceux-ci manquent d'un axe intrapersonnel qui permette l'observation de la même personne dans diverses situations et d'une transcription phonétique.

Pour cette raison, nous avons créé la base de données T-zéro, qui permet d'abord le double contraste sur l'axe diatopique (Suisse ${ }^{77}$ vs. France) et intrapersonnel (c'est-à-dire entre les mêmes locuteurs dans deux situations de communication), puis d'éclairer sous un nouveau jour l'influence de la réalisation phonétique des clitiques sur $\pm n e$.

T-zéro permet le stockage et l'analyse de trois types de données: a) linguistiques, b) sociodémographiques et c) situationnelles. Celles-ci figurent respectivement dans les trois masques de saisie qui sont représentés en (99): le masque vert comporte les données linguistiques, le masque rose est réservé aux données sociodémographiques et le jaune décrit les situations de communication.

(99) Les types de données stockées dans T-zéro

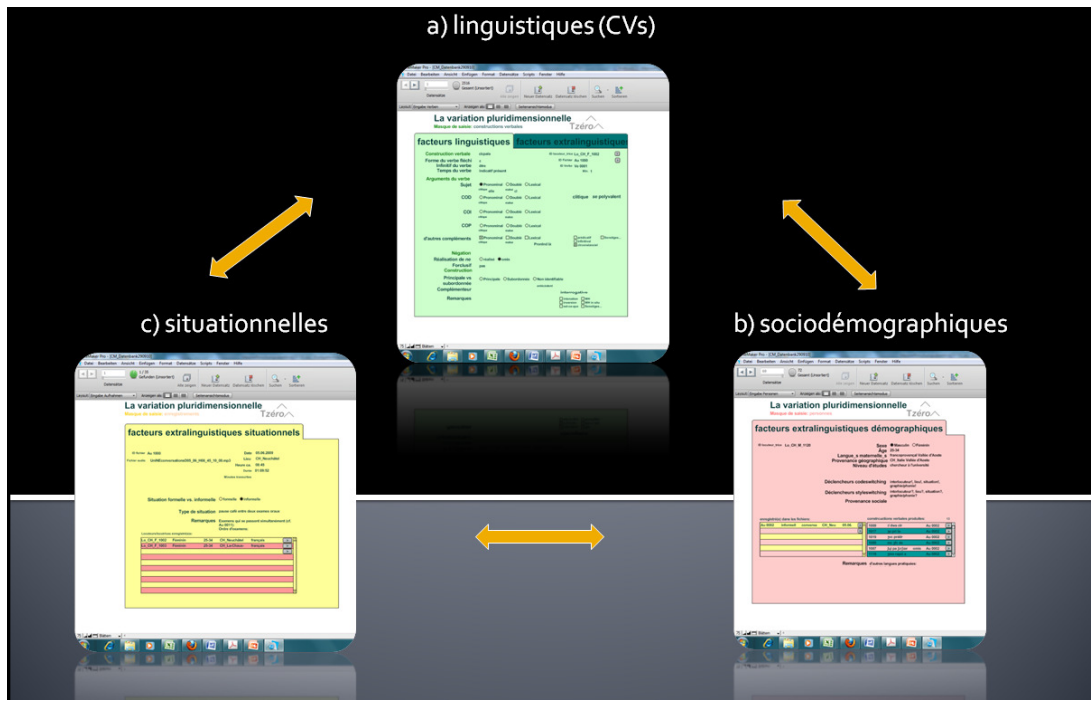

77 Les situations de communication enregistrées pour le PFC en Suisse (un texte $\mathrm{lu}$, une liste de mots, une discussion libre et une discussion guidée) ne correspondent pas aux pôles de l'immédiat et de la distance. 
Les masques de saisie sont interconnectés et la structure de la base de données permet d'opérer un changement dynamique entre les différents masques, voire entre les types de données. Ceci permet de choisir (au moins) trois points de départ différents pour une analyse. De cette façon, en partant d'une construction verbale (CV) déterminée, cf. (99)a, nous pouvons examiner les données sociodémographiques du locuteur qui l'a produite ou la comparer avec d'autres énoncés produits dans la même situation. Il est également possible de partir d'un locuteur et de comparer sa production langagière dans différentes situations. Finalement, nous pouvons choisir une situation déterminée et nous intéresser aux différents locuteurs enregistrés ou aux CVs qui ont été produites dans cette situation. Les résultats des requêtes peuvent être exportés sous divers formats ${ }^{78}$, ce qui permet l'analyse à l'aide de logiciels statistiques comme Excel, SPSS ou R.

\subsection{Description sociodémographique de T-zéro}

T-zéro contient les données sociodémographiques de 72 locuteurs et locutrices, dont la majorité provient de France ${ }^{79}$. Toutefois, le déséquilibre ${ }^{80}$ géographique n'altère aucunement les données linguistiques

$78 \quad$ *.tab; *.csv; *.mer; *.htm; *.fp7; *.xml; *.xls; *.xlsx.

79 L'expression de provenance se réfère ici au pays dans lequel les locuteurs habitent au moment des enregistrements. Pour la majorité des locuteurs, cet endroit correspond au lieu de naissance et d'enfance, mais il existe cependant quelques exceptions, signalées dans le champ remarques du masque de saisie rose.

80 Nous tenons à signaler que la répartition entre les hommes et les femmes est inégale. Il y a une présence relativement faible de CVs produites par des hommes (seulement $23 \%$ en $\mathrm{FR}$ et $\mathrm{CH}$ au total), ce qui est dû à trois facteurs: premièrement, il y a généralement une faible représentation d'hommes dans la population enregistrée (au moment de l'enregistrement, la quantité de femmes était nettement supérieure à celle des hommes, et cela aussi bien dans les deux lycées d'Île-de-France qu'à l'institut universitaire de Neuchâtel); deuxièmement, les hommes parlent moins (du moins pendant les enregistrements), en effet, dans 
brutes, car les locuteurs suisses, bien que numériquement inférieurs, manifestent une production linguistique très active. Ainsi, T-zéro permet la comparaison de quantités presque égales de données françaises et suisses:

(100) Situations et lieux d'enregistrement dans T-zéro
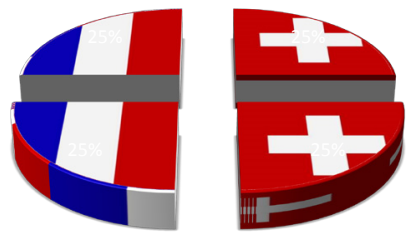

\author{
t Examen $\mathrm{CH}$ \\ t Examen FR \\ II Conversation FR \\ II Conversation $\mathrm{CH}$
}

La démographie de T-zéro s'explique par l'objectif du projet de comparer des situations d'examens avec des conversations et des discussions. Les situations d'examen se produisent dans le milieu universitaire et scolaire, où plusieurs étudiants et élèves sont interrogés par un nombre restreint de professeurs ou chargés de cours. La majorité des locuteurs enregistrés sur T-zéro est donc constituée par des jeunes de 14 à 18 ans suivis par des jeunes adultes de 19 à 24 ans. On compte six locuteurs de entre 25 à 34 ans et les groupes de 35 ans et plus ne se composent que d'une seule personne par groupe. Ce sont les groupes des jeunes gens de 18 à 24 ans qui produisent la majorité des données linguistiques stockées dans T-zéro.

La plupart des locuteurs enregistrés sont des lycéens d'Île-deFrance et des étudiants universitaires de Neuchâtel, tandis qu'une minorité de locuteurs est constituée par des professeurs de lycée, des assistants-doctorants et un professeur d'université. Dans l'ensemble, les

T-zéro, la part des CVs produites par des hommes (23\%) est inférieure à la part des hommes enregistrés (33\%); finalement, la création d'un corpus est toujours assujettie à la participation des volontaires qui se font enregistrer. Toutefois, la représentation inégale des sexes dans T-zéro est reflétée de manière uniforme aussi bien en Suisse qu'en France et les comparaisons entre les deux pays quant au comportement linguistique des deux sexes restent ainsi possibles. 
locuteurs jeunes dominent clairement la structure d'âge de T-zéro ${ }^{81}$, même si ce déséquilibre est quelque peu contrecarré par une production orale plus active des locuteurs âgés dans leur fonction d'examinateurs.

Étant donné que la majorité des locuteurs n'exerce aucune profession salariée, les revenus des participants n'ont pas été retenus. Toutefois, la provenance géographique exacte des locuteurs nous permet de retenir quelques informations concernant la structure sociale de la population enregistrée.

(101) La provenance géographique et sociale des locueurs ${ }^{82}$

a. Carte de l'Île-de-France

b. Carte du canton de Neuchâtel
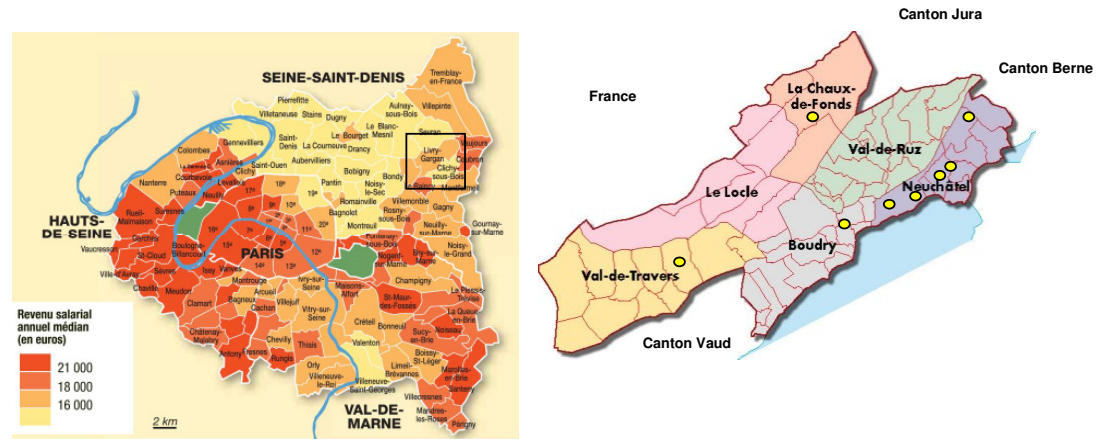

Selon l'Institut national de la statistique et des études économiques (INSEE) de France, en 2008, le revenu moyen par ménage à Clichysous-Bois était de $18^{\prime} 586.51$ Francs Suisses $\left(=15^{\prime} 541\right.$ Euro $\left.^{83}\right)$ par an et comptait parmi les plus faibles en Île-de-France en $2011^{84}$. Quant au

81 Nous tenons à noter que T-zéro offre l'option d'inclure ou d'exclure, pour les analyses, certains groupes d'âge ainsi que des groupes formés en fonction de n'importe quel autre facteur démographique.

82 Sources des cartes: <http://fr.wikipedia.org/wiki/Fichier:Revenus_\%C3\%A0_ Paris_et_Petite_Couronne.JPG $>$, et $<$ http://www.surface.ch/ne/map-district.htm $>$, consultées le 18 mars 2011.

83 Calculé à l'aide du taux du change du 29 juin 2011 (1 EUR = $1.19612 \mathrm{CHF}$ ).

$84 C f .<$ http://www.statistiques-locales.insee.fr/FICHES/RS/DEP/93/COM/RS_COM 93014.pdf>, consulté le 07 mars 2011. 
taux de chômage dans cette commune, il est de $21,2 \%$ en $2007^{85}$. À Livry Gargan, en revanche, le revenu moyen annuel en 2008 est de 28 893.53 CHF ( $=24{ }^{\prime} 156$ Euro $\left.^{86}\right)$, dépassant ainsi la moyenne du département de Seine-Saint-Denis, dont les deux communes font partie ${ }^{87}$, qui s'élève à $23,625.32 \mathrm{CHF}\left(=19^{\prime} 749\right.$ Euro $\left.^{88}\right)$ par an. Dans cette commune, le taux de chômage s'élève à $9,5 \%$ en $2007^{89}$.

En Suisse romande, par contre, le revenu moyen annuel par ménage s'élève à $78^{\prime} 096^{90} \mathrm{CHF}$ et le taux de chômage dans le canton de Neuchâtel est de $6,4 \%{ }^{91}$ en 2010. Un ménage moyen en Suisse romande gagne donc en trois mois environ le même montant qu'un ménage moyen à Clichy-sous-Bois en un an. En termes de revenus moyens, nous constatons donc que les locuteurs suisses que nous avons enregistrés sont nettement plus aisés que ceux français. Cependant, il est nécessaire de noter que le coût de la vie en Suisse est l'un des plus élevés en Europe ${ }^{92}$ : selon Eurostat, les coûts des produits alimentaires, du logement et de la santé sont entre $30 \%$ et $60 \%$ plus élevés par rapport à la moyenne européenne. En France, les mêmes coûts ne dépassent la moyenne européenne que de $3 \%$ à $24 \%$.

Compte tenu de ces chiffres, une comparaison directe du niveau social entre les deux points d'enregistrement en France, à savoir Clichysous-Bois et Livry Gargan, et le point d'enregistrement à Neuchâtel,

$85 C f .<$ http://www.statistiques-locales.insee.fr/FICHES/DL/DEP/93/COM/DL_COM 93014.pdf $>$, consulté le 07 mars 2011.

86 Calculé à l'aide du taux de change du 29 juin 2011 (1 EUR = $1.19612 \mathrm{CHF})$

$87 C f .<$ http://www.statistiques-locales.insee.fr/FICHES/RS/DEP/93/COM/RS_COM 93046.pdf >, consulté le 07 mars 2011.

88 Calculé à l'aide du taux de change du 29 juin 2011 (1 EUR = $1.19612 \mathrm{CHF}$ ).

$89 C f .<$ http://www.statistiques-locales.insee.fr/FICHES/DL/DEP/93/COM/DL_COM 93046.pdf>, consulté le 07 mars 2011.

90 Le revenu mensuel en Suisse romande entre 2006 et 2008 était de 6508 Francs suisses selon l'office fédéral de la statistique, $c f .<$ http://www.bfs.admin.ch/bfs/ portal/fr/index/themen/20/02/blank/key/einkommen0/niveau. Document.137707. xls>, consulté le 18 mars 2011.

$91<$ http://www.bfs.admin.ch/bfs/portal/fr/index/themen/03/03/blank/key/registrierte_arbeitslose/nach kanton.html>, consulté le 18 mars 2011.

$92<$ http://www.travailler-en-suisse.ch/html/cout-vie-en-suisse.html $>$, consulté le 18 mars 2011. 
en Suisse romande, semble difficile, voire impossible. D’une manière générale, nous pouvons toutefois conclure que les conditions de vie à Clichy-sous-Bois sont précaires, à Livry Gargan défavorisées et à Neuchâtel relativement bonnes.

En ce qui concerne les langues maternelles des locuteurs, nous observons dans le diagramme (102) que la majorité (81\%) indique uniquement le français comme langue maternelle.

(102) Les langues maternelles des locuteurs dans T-zéro

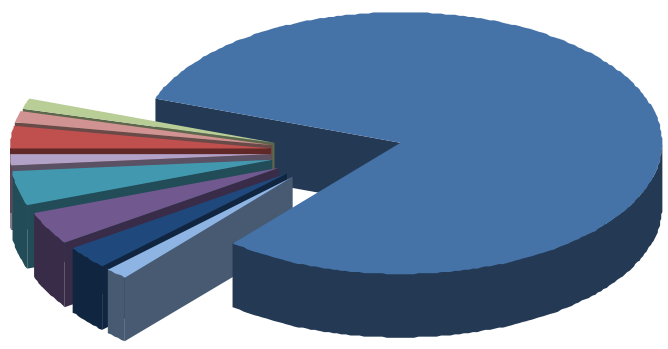

$$
\begin{aligned}
& \text { français } \\
& \text { fr }+ \text { anglais } \\
& \text { - } \mathrm{fr}+\text { suisse-allemand } \\
& \square \mathrm{fr}+\text { arabe } \\
& \square \mathrm{fr}+\mathrm{furc} \\
& \text { fr + 'camerounais' } \\
& \square \text { suisse-allemand } \\
& \square \text { créole martiniquais } \\
& \text { francoprovençal Vallée d'Aoste }
\end{aligned}
$$

Dans le diagramme (102), nous trouvons une liste des autres langues qui ont été indiquées comme langues maternelles. Certains locuteurs parlent le francoprovençal, le créole martiniquais ou le suisse allemand comme L1 et ont appris le français comme L2. Néanmoins, comme ils habitent dans une région francophone, leur performance est comparable à celle des natifs. D'autres locuteurs sont bilingues et indiquent, en combinaison avec le français, le turc, l'arabe, le suisse-allemand ou l'anglais comme seconde langue maternelle. Dans l'ensemble, le français est la langue maternelle prépondérante du corpus, mais les locuteurs bilingues et de L2 avancés forment un sous-ensemble important d'environ $20 \%$. Nous avons inclus ces locuteurs dans notre corpus, car le plurilinguisme est désormais une réalité dans les régions francophones. Ce qui revient à dire que, surtout dans les régions étudiées ici, les locuteurs bilingues et non-natifs contribuent eux aussi à la variation et aux changements linguistiques du français. 


\subsection{Description des situations d'enregistrement}

La dimension intrapersonnelle de T-zéro concerne la variation entre les différentes situations de communication. Nous visons, de cette façon, à caractériser les situations de communication enregistrées dans le cadre du modèle de Koch/Oesterreicher (2001, $\left.{ }^{2} 2011\right)$. Ce modèle permet la combinaison libre de divers paramètres communicatifs et fournit ainsi une base idéale pour la description des situations de communication qui nous intéressent. Dans le continuum entre l'immédiat et la distance communicative en (103), les situations pertinentes pour notre analyse apparaissent en caractères gras.

(103) Le continuum entre l'immédiat et la distance communicative (face-toface $^{93}$ )

Immédiat communicatif

Distance communicative

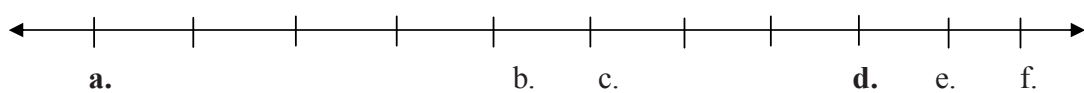
a. conversation spontanée entre amis [ou connaissances, C.M.]
b. entretien professionnel
c. interview de presse ${ }^{94}$
[d. examen oral, C.M.]
e. sermon
f. conférence scientifique
g. lecture à haute voix d'un texte de loi
(cf. Koch/Oesterreicher 2001: 585-586)

93 Koch/Oesterreicher (2001: 586) mentionnent également la situation coup de téléphone, qui a été supprimée ici, car nous nous intéressons à la communication directe, i. e. face-to-face, (cf. aussi la discussion dans Meisner 2010: 1952 sur la non-pertinence du facteur téléphone pour la réalisation de $n e$ ).

94 Koch/Oesterreicher (2001: 586) considèrent l'interview de presse de manière résultative, c'est-à-dire uniquement comme forme de réalisation graphique (par exemple dans les journaux). Par contre, nous nous référons au processus de l'interrogation et considérons donc la situation de l'interview comme conversation phonique. 
Deux situations du continuum apparaissent dans notre analyse: la situation la plus proche du pôle de l'immédiat, c'est-à-dire la conversation spontanée entre amis ou connaissances, et la situation de l'examen oral, que nous avons ajoutée au continuum de Koch/Oesterreicher (2001: 585-586) et qui représente, dans notre analyse, le domaine de la distance communicative. Dans l'ensemble, T-zéro comprend quatre types de situations, qui sont représentés de manière quantitativement équilibrée:

(104) Les types de situation présents dans le corpus

\begin{tabular}{lllll}
\hline & Distance & Immédiat & \\
\hline Suisse & Examen oral à l'université & $25 \%$ & Conversations entre amis/connaissances & $25 \%$ \\
France & Examen oral au lycée & $25 \%$ & Conversations entre amis/connaissances & $25 \%$ \\
\hline
\end{tabular}

Une moitié des données linguistiques appartient au domaine de la distance communicative et l'autre à celui de l'immédiat communicatif. Les premières ont été enregistrées lors d'examens oraux et les dernières pendant des conversations et discussions entre amis et connaissances ou avec la chercheuse, que les élèves connaissent en tant qu'assistante de langue. Une moitié des données a été collectée en France et l'autre en Suisse.

\subsubsection{La distance communicative: l'examen oral}

L'examen oral a été choisi comme situation exemplaire de la distance communicative. Cette situation unit, malgré la présence de certains aspects de l'immédiat communicatif, un nombre élevé de caractéristiques du domaine de la distance. Voici le relief conceptionnel de cette situation, établi d'après le modèle de Koch/Oesterreicher (2001, 2011): 
(105) Le relief conceptionnel de l'examen oral

\begin{tabular}{l}
\hline (1) Communication privée \\
(2) Interlocuteur intime \\
(3) Émotionnalité forte \\
(4) Ancrage actionnel et \\
situationnel \\
(5) Ancrage référentiel dans \\
la situation \\
(6) Coprésence spatio- \\
temporelle \\
(7) Coopération \\
communicative intense \\
(8) Dialogue \\
(9) Communication \\
spontanée \\
(10)Liberté thématique \\
immédiat
\end{tabular}

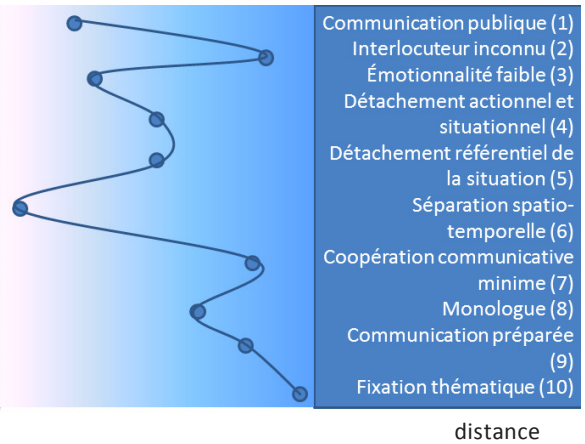

Le côté gauche de la figure (105) montre l'ensemble des caractéristiques de l'immédiat communicatif, tandis que le côté droit unit les traits typiques de la distance communicative. Le caractère graduel de neuf des dix paramètres (seule la coprésence spatio-temporelle est binaire) permet l'établissement d'un profil communicatif individuel pour les différentes situations enregistrées.

Pour la situation de l'examen oral, la majorité des paramètres est fixée plutôt proche du pôle de la distance communicative. Malgré ce classement général, le relief manifeste trois valeurs qui se trouvent dans le domaine de l'immédiat, à savoir, les paramètres (1) communication privée, (3) émotionnalité forte et (6) coprésence spatio-temporelle. Le fait, éventuellement surprenant, que les situations d'examen sont classées comme 'privées' dépend de la définition du paramètre privé vs. public par Koch/Oesterreicher (2011: 17) ${ }^{95}$ : dans le cas de l'examen oral, il serait éventuellement plus clair de parler d'une communication non-publique que d'une communication privée. Vu que les candidats peuvent être stressés lors d'un examen oral, car ils soumis à une grande

95 Les examens oraux ont lieu en présence d'un public très restreint: seuls les candidats et un ou deux professeurs ou chargés de cours sont présents. La situation est donc classée, selon les critères de Koch/Oesterreicher (22011: 7), comme une communication privée. 
pression et se trouvent dans une situation de compétition, nous fixons le paramètre de l'émotion un peu plus proche du pôle de l'immédiat que de celui de la distance communicative ${ }^{96}$.

Quant au paramètre (2), les relations entre les interlocuteurs sont caractérisées par la hiérarchie institutionnellement fixée: ainsi, les chercheurs se trouvent sous la tutelle des professeurs universitaires et ces deux groupes dominent les étudiants. Similairement, les professeurs scolaires sont des personnes hiérarchiquement supérieures par rapport aux élèves. De plus, les candidats et les examinateurs ne se connaissent pas dans tous les cas. Par conséquent, ce paramètre est l'un des plus proches du pôle de la distance.

La variété linguistique envisagée pendant les examens est clairement le français standard. Toutefois, la production verbale spontanée est soumise aux effets de performance (reformulations, interruptions, pauses etc.). Les paramètres (4) et (5) de l'ancrage situationnel, actionnel et référentiel sont classés au niveau intermédiaire entre les pôles de l'immédiat et de la distance, car les examens portent d'un côté sur des extraits de texte et des matériaux que les candidats ont sous les yeux, ce qui implique un certain ancrage dans la situation concrète, alors que d'autres questions dépassent la situation de l'examen en faisant référence à des cours antérieurs.

La coopération communicative (7) est plutôt réduite, du moins du côté de l'examinateur. De plus, à cause des rôles clairement distribués entre les deux participants (l'examinateur pose les questions; le candidat y répond), le système de prise de parole, caractéristique d'un dialogue (8), est également fortement réglementé. Même si le déroulement détaillé de l'examen reste imprévisible pour tous les participants, il s'agit toutefois d'une communication bien préparée à l'avance (9), dont la fixation thématique (10) est très élevée.

96 Il faut noter que l'attitude des candidats dans une telle situation varie considérablement en fonction de chaque individu: certains ont le trac ou sont angoissés, d'autres s'agitent ou s'énervent et d'autres encore se montrent disciplinés, détendus ou impassibles. 
Dans l'ensemble, et contrairement aux discours fortement ritualisés, qui sont typiques de la distance communicative, comme un sermon, une conférence scientifique ou une lecture à haute voix d'un texte de loi (cf. Koch/Oesterreicher 2001: 585-586), l'examen oral offre la possibilité idéale d'observer un langage dialogique et spontané, même dans le domaine de distance.

\subsubsection{L'immédiat communicatif: conversations et discussions}

Le domaine de l'immédiat est représenté par deux situations de communication: d'une part, les conversations libres entre deux et six personnes (élèves, étudiants et chercheurs) et d'autre part, les discussions en groupe qui ont eu lieu pendant les cours de la chercheuse, qui travaille comme assistante d'allemand dans les deux lycées en France (17\% du corpus).

Les conversations ont lieu dans une salle d'attente ou dans une petite cafétéria avant et après les examens. Celles-ci se caractérisent par le profil communicatif en (106), qui est relativement proche du pôle de l'immédiat.

(106) Le relief conceptionnel des conversations

(1) Communication privée
(2) Interlocuteur intime
(3) Émotionnalité forte
(4) Ancrage actionnel et
situationnel
(5) Ancrage référentiel dans
la situation
(6) Coprésence spatio-
temporelle
(7) Coopération
communicative intense
(8) Dialogue
(9) Communication
spontanée
(10)Liberté thématique

immédiat

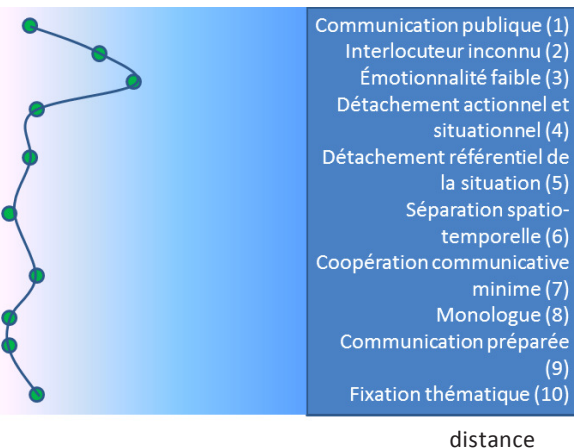


Les conversations sont menées par un nombre restreint d'interlocuteurs et ont donc lieu dans un cadre privé (1). Les degrés d'intimité (2) entre les interlocuteurs sont variés: les élèves se voient tous les jours et se connaissent plus ou moins bien, et la plupart des étudiants se fréquente depuis environ un an. Les chercheurs sont des collègues qui travaillent ensemble pendant des périodes variées et certains d'entre eux entrent également en contact privé. Dû à sa complexité, le réseau des contacts entre les interlocuteurs est difficile à déterminer et, par conséquent, nous nous limitons à noter une complicité élevée à l'intérieur des groupes d'élèves, d'étudiants et de chercheurs. Les thèmes de certaines conversations suggèrent effectivement l'existence d'amitiés profondes parmi certains participants, tandis que d'autres semblent être de simples connaissances. Vu la nature hétérogène des relations entre les participants de l'étude, nous fixons le paramètre à la moitié de l'immédiat, mais pas trop près de ce pôle.

Les émotions (3) montrées par les interlocuteurs dépendent du contexte concret, mais celles-ci sont généralement moyennes. L'ancrage actionnel, situationnel et référentiel des conversations est plus fort (4) que dans les examens: les discussions tournent souvent autour des référents immédiatement présents (les personnes, les examens passés ou à venir, les objets sous les yeux etc.). Les sujets discutés varient librement (5) et concernent tous les aspects de la vie scolaire ou estudiantine quotidienne: les examens passés, les projets pour l'été ou pour le week-end, des petites anecdotes privées, les examens et les cours de l'année académique ainsi que les rumeurs concernant les professeurs et les autres élèves ou étudiants.

Le relief conceptionnel des discussions sous (107) ressemble fortement à celui des conversations. La plupart des paramètres sont fixés dans la moitié gauche de la figure, d'où découle le classement général comme situation de l'immédiat communicatif. 
(107) Relief conceptionnel des discussions en classe

(1) Communication privée
(2) Interlocuteur intime
(3) Émotionnalité forte
(4) Ancrage actionnel et
situationnel
(5) Ancrage référentiel dans
la situation
(6) Coprésence spatio-
temporelle
(7) Coopération
communicative intense
(8) Dialogue
(9) Communication
spontanée
(10)Liberté thématique

immédiat

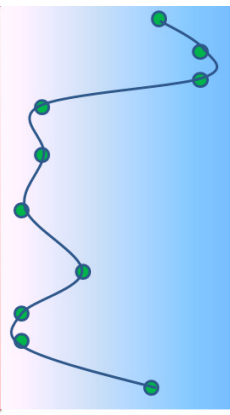

Communication publique (1)

Interlocuteur inconnu (2)

Émotionnalité faible (3)

Détachement actionnel et

situationnel (4)

Détachement référentiel de

la situation (5)

Séparation spatio-

temporelle (6)

Coopération communicative

minime (7)

Monologue (8)

Communication préparée

Fixation thématique (10)

distance

Les cours ont lieu en petits groupes de 5 à 10 élèves, qui se voient tous les jours et se connaissent bien, et dans une atmosphère détendue, conçue intentionnellement comme contrepoids aux autres cours scolaires très stricts. Le paramètre (1) est donc fixé au milieu entre les deux pôles de la communication privée et publique.

Néanmoins, et contrairement aux conversations, les discussions sont surveillées par une assistante de langue, la chercheuse, qui fait partie du corps enseignant, et qui reste donc un personnage respectable pour les élèves. Généralement, les émotions montrées par les interlocuteurs sont modérées, mais elles peuvent varier en fonction du sujet discuté ${ }^{97}$. Par conséquent, les paramètres (2) et (3) sont fixés au milieu du continuum.

L'ancrage actionnel, situationnel (4) et référentiel (5) est plutôt fort, car les discussions portent souvent sur des matériels présents dans la salle de cours ou sur les actions des interlocuteurs mêmes. Comme cela est typique dans le domaine de l'immédiat, les interlocuteurs sont également coprésents (6) pendant les discussions. La conception du cours prévoit d'inspirer la confiance des élèves et de cultiver leurs capacités d'expression libre, ce qui implique une coopération communicative élevée (7). Les sujets abordés pendant les cours concernent tous

97 Les discussions concernant les sujets comme la religion et l'amour ont parfois provoqué des réactions très fortes de la part des élèves. 
les aspects de la vie quotidienne des adolescents: l'amour, la littérature et la musique allemande ainsi que la politique ou l'actualité. Toutefois, la liberté thématique des cours est restreinte par rapport aux conversations, puisque les matériels mis en place par l'assistante imposent des thématiques concrètes pour la discussion. Le paramètre (10) est, par conséquent, classé comme moyen.

En résumant, les conversations et les discussions, qui représentent le domaine de l'immédiat communicatif, manifestent des reliefs conceptionnels similaires mais pas identiques. Ceci permet, d'une part, d'analyser un domaine communicatif dans sa profondeur et, d'autre part, de détecter d'éventuelles micro-différences entre les deux types de situations.

\subsection{Le protocole d'enregistrement}

Notre position en tant qu'assistante d'allemand ${ }^{98}$ (octobre 2007 - mars 2008) dans deux lycées d'Île-de-France, plus précisément à Livry Gargan et à Clichy-sous-Bois, nous a permis de gagner la confiance du rectorat, des professeurs et des élèves, et de les motiver à participer aux enregistrements des situations d'examen ${ }^{99}$ et de conversations privées ou en classe. Par la suite, une coopération entre l'institut des langues romanes de l'Université de Zurich et l'Université de Neuchâtel a permis d'effectuer un enregistrement similaire le 4 et 5 juin 2009 en Suisse. Dans l'ensemble, 48 heures de production langagière ont été enregistrées et transcrites partiellement (cf. section 3.7): 28.28 heures en Îlede-France et 19.69 heures à Neuchâtel.

98 Des informations détaillées sur le programme des assistants de langue sont à trouver sous: <http://www.kmk-pad.org/programme/dtsch-fsa.html>.

99 Seul l'un des deux lycées ayant participé au projet a autorisé l'enregistrement d'examens oraux. Tous les enregistrements français d'examens oraux proviennent donc du lycée de Livry Gargan. 
Après le consentement des responsables au niveau scolaire et universitaire, nous avons présenté le projet de recherche aux locuteurs restants. Ceux qui souhaitaient y participer ont alors signé une déclaration de consentement pour l'enregistrement d'un examen et/ou d'une conversation et rempli un questionnaire sociolinguistique ( $c f$. Baude 2007). Les locuteurs ont été informés qu'il s'agissait d'un projet purement linguistique, que ni le contenu ni la note de l'examen n'auraient une influence sur l'analyse, que la participation était anonyme et volontaire, et que toutes les données personnelles resteraient confidentielles et seraient anonymisées avant la publication. Si les locuteurs étaient mineurs au moment de l'enregistrement, les parents ont signé la déclaration à leur place.

Après la procédure d'information et de consentement, les enregistreurs ont été installés, aussi discrètement que possible, au milieu des salles et directement allumés avant le début de l'examen ou de la conversation.

Le paradoxe de l'observateur (cf. Labov 1972b: 209) a pu être partiellement franchi par le fait que la chercheuse s'est absentée pendant l'enregistrement des examens et que les élèves la connaissaient bien et ne la ressentaient donc pas comme un intrus dans leur vie scolaire quotidienne. De plus, certains élèves ont assumé que l'intérêt scientifique d'une chercheuse allemande ne pouvait porter que sur la langue allemande et ainsi, bon nombre de participants se sont forcés davantage à 'bien' parler en allemand, sans accorder beaucoup d'attention à leur production linguistique en français, ce qui correspond parfaitement à l'objectif de notre étude. Néanmoins, le risque minimal de toute analyse de corpus persiste, à savoir que le comportement observé des locuteurs ne soit pas identique à celui inobservé. 


\subsection{Le protocole de transcription}

La majorité des corpus oraux modernes est transcrite dans une orthographe standard adaptée aux particularités de la langue phonique (cf. Baude 2006, Bilger 2008, Cresti/Moneglia 2005, Durand et al. 2005). Ceci signifie que les éléments absents, comme par exemple les ne de négation ou les explétifs supprimés, ne sont généralement pas restitués:

(108) La transcription en orthographe standard adaptée

a. je pense pas qu'il y en ait une description où les corps sont autant décrits (0288)

b. $\quad y$ a tout ce qu'il faut (0002)

Nous avons adapté cette pratique bien établie pour la transcription de notre corpus, mais, en même temps, nous sommes allés encore plus loin. Dans T-zéro, chaque entrée en orthographe standard est redoublée par une transcription en alphabet phonétique international (API):

(109) La transcription en API ${ }^{100}$

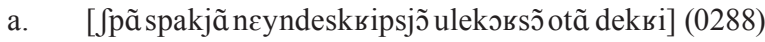

b. [jatuskifo] (0002)

Tant la lisibilité des données comme l'exactitude de la transcription sont assurées par cette double transcription. La transcription orthographique, qui a l'avantage de présenter les données sous une forme bien connue et facilement déchiffrable, présente l'inconvénient de dissimuler la forme exacte sous laquelle certains éléments grammaticaux sont réalisés, ce qui vaut avant tout pour les clitiques. En effet, si nous comparons la représentation du pronom je en (108)a et (109)a, nous observons que la transcription orthographique je pense ne témoigne rien sur la forme [ $\left.\int \mathrm{p} \tilde{\mathrm{s}} \mathrm{s}\right]$ qui est réellement produite. Bien évidemment, l'orthographe standard permettrait la représentation de certaines va-

100 Je tiens à remercier Elissa Pustka pour ses suggestions utiles quant à la transcription phonétique. 
riantes de clitiques, comme par exemple j'pense, mais cette transcription serait toujours moins exacte que celle en API. Seule cette dernière permet d'expliciter que la variante [ $\left.\int\right]$ pour je comporte non seulement l'omission du schwa [3ə] $\rightarrow$ [3], mais également l'assourdissement de la fricative post-alvéolaire [3] $\rightarrow\left[\int\right]$.

La transcription en API est une transcription phonétique acoustique étroite, c'est-à-dire que nous représentons le plus fidèlement possible le signal acoustique discerné par les transcripteurs à l'aide des symboles de l'API, mais sans signes diacritiques. Ceci signifie que la réalisation des clitiques, les contractions clitique+verbe, les élisions vocaliques, les omissions de consonnes finales, le degré d'ouverture des voyelles $\mathrm{E}$ et $\mathrm{O}$, et les géminés sont transcrits selon les conventions de la phonétique acoustique.

En revanche, la prosodie, les signaux de l'organisation de la prise de parole (euh, hmm) et les marqueurs de discours et d'autre matériel lexical syntaxiquement non-intégré et non-adjacent à une construction verbale ne sont pas transcrits. La transcription phonétique ne comprend donc pas d'espace blanc. Les pauses longues sont marquées par une barre, tandis que les micro-pauses ne sont pas transcrites (sauf si elles semblent pertinentes pour l'analyse). Afin de documenter un maximum de locuteurs, nous avons décidé de transcrire seulement quelques minutes par enregistrement. La transcription porte uniquement sur les parties dialogiques menées en français, tandis que les lectures d'extraits de textes et les fragments de discours uniquement en langue allemande sont supprimés. Le protocole de transcription complet, comprenant des exemples pour les deux systèmes de transcription, se trouve dans l'annexe V.

Compte tenu des déficits de la transcription orthographique par rapport à celle phonétique, l'on pourrait alors se demander pourquoi la première est tout de même appliquée. Cela s'explique par le fait que nous avons constaté qu'une simple transcription phonétique n'est pas suffisante non plus. En effet, même les lecteurs expérimentés ont du mal à lire des passages moyens ou longs en API, comme celui dans l'exemple (109). De plus, la recherche dans le corpus serait très pénible: comme nous ne pouvons pas connaître d'avance toutes les variantes phonétiques d'une forme, il serait difficile, voire impossible de les trouver par une simple 
requête. La combinaison des deux systèmes de transcription permet donc de compenser les inconvénients de chacun d'entre eux et de créer ainsi une base de données solide pour l'analyse envisagée.

\subsection{Le protocole pour l'analyse de la variable $\pm n e$ dans T-zéro}

Afin d'identifier les données de base pour l'analyse de corpus, une requête qui porte sur toutes les constructions verbales contenant un terme négatif est lancée sur T-zéro. À l'aide d'une recherche dans Tzéro, Filemaker fait apparaître une liste de toutes les entrées dans lesquelles ce champ a été rempli. L'export des données sous forme d'un fichier Excel est possible. Toutes les données linguistiques et extralinguistiques spécifiées par rapport aux entrées négatives figurent dans le tableau exporté.

La dernière étape avant de commencer l'analyse consiste en la suppression des constructions négatives dans lesquelles l'on ne distingue pas clairement si ne est réalisé ou non. Ceci signifie que tous les cas où la réalisation de ne pourrait être confondue avec une liaison, comme par exemple dans on (n')a pas vu [õ napavy], sont exclus du calcul. À ce sujet, Fonseca-Greber (2007) constate qu'il serait possible de distinguer à l'aide de la longueur du son [n] s'il s'agit d'une liaison ou d'une particule de négation:

(110) With respect to the on $\pm n e$ sequences [...] it appears possible to distinguish between the on -ne sequences and geminated on $+n e$ sequences in pre-vocalic contexts, given the added length in the geminate sequences. (Fonseca-Greber 2007: 256)

Fonseca-Greber (2007) suggère que les [n] de négation qui suivent on seraient 'géminés' et, par conséquent, plus longs que les [n] de liaison, mais cette hypothèse n'a pas été confirmée lors de notre analyse. 
Au niveau phonologique, il semble peu convainquant que les séquences on+ne soient réellement géminées. Si ne est produit, comme en (111)a, il n'y a aucune liaison, car le pronom on est suivi par la consonne [n] du ne et la consonne latente [n] dans on reste muette. Autrement dit, la liaison est bloquée par la présence de ne. Par contre, si ne est absent, comme en (111)b, une liaison obligatoire est produite, déclenchée par la voyelle [a] qui suit on.
(111) La liaison dans les séquences on $\pm n e$
a. on + ne: on \# n'a pas [כ̃napa]
b. on-ne: on [liaison] a pas [J̃napa]
c. on + ne: ${ }^{*}$ on $_{\text {[liaison] }}$ n'a pas $*$ [J̃nnapa]

Le troisième cas décrit par Fonseca-Greber (2007) et illustré en (111)c, à savoir la coprésence des deux [n] de négation et de liaison, est, à notre avis, agrammatical et ne se produit jamais. Nous soutenons, par contre, que les [n] du ne et de la liaison apparaissent en distribution complémentaire dans les contextes prévocaliques:

(112) La distribution complémentaire des [n] de négaion et de liaison

\begin{tabular}{|ll|}
\hline \multicolumn{2}{|l|}{ Contexte: $\pm n e \mathrm{~V}$} \\
\hline tne & -liaison \\
-ne & +liaison \\
\hline
\end{tabular}

Il n'y a donc aucune motivation phonologique à assumer une différence phonétique entre les [n] de négation et de liaison.

Néanmoins, l'on peut se demander s'il ne serait quand-même pas possible que les [n] de négation soient effectifvement plus longs, comme le suggère Fonseca-Greber (2007). Afin d'établir si une telle différence de longueur existe, nous avons comparé les deux types de [n] à l'aide du lociciel d'analyse phonétique PRAAT ${ }^{101,102}$. Les figures en (113) montrent que le [n] qui apparait dans une construction sans négation, représenté sous forme de barre noire en (113)a, est même un

$101 C f .<$ http://www.fon.hum.uva.nl/praat/>.

102 Je remercie Stephan Schmid pour son précieux conseil quant à cette comparaison. 
peu plus long que le [n] dont le statut reste ambigu et oscille entre celui de négation et de liaison en (113)b.

(113) Comparaison des [n] prévocaliques avec et sans négation

a. $\quad$ sans négation: on a [J̃na]

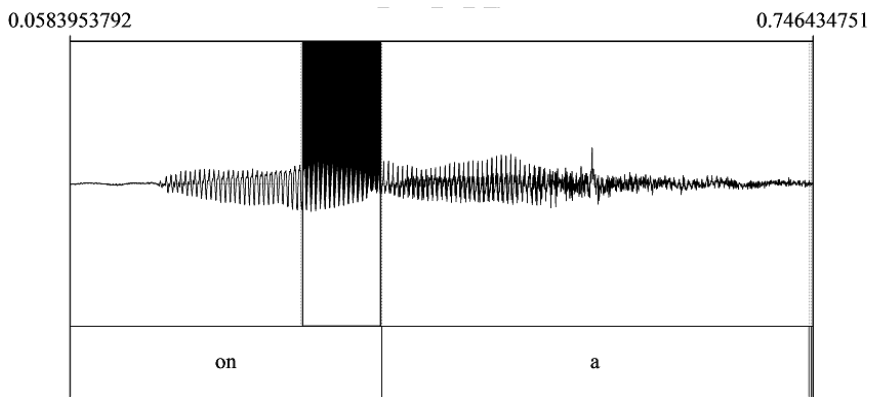

b. $\quad$ avec négation: on (n')a plus [ว̃naply]

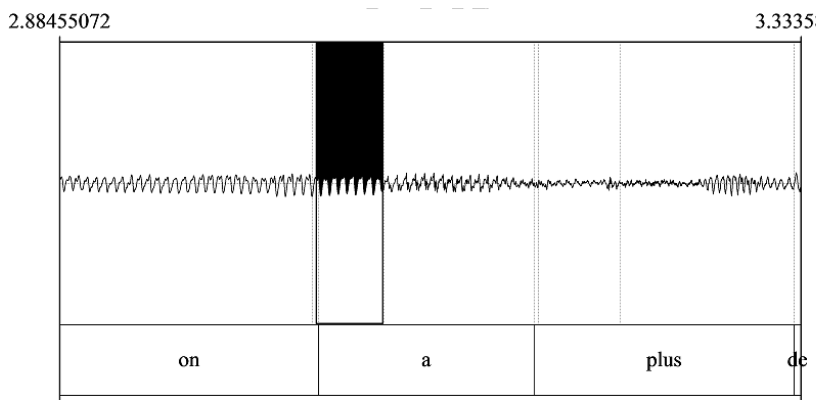

A notre avis, il n'y a donc aucun moyen de distinction entre les [n] de négation et ceux de liaison dans les contextes prévocaliques négatifs. Par conséquent, et suivant la pratique d'Ashby (1976), (1981), (2001), Armstrong (2002), Armstrong/Smith (2002), Coveney (22002), Dufter/ Stark (2007) et Hansen/Malderez (2004), ces cas ambigus sont exclus de notre analyse. 


\subsection{Les tests statistiques pour l'analyse descriptive}

Conformément aux pratiques courantes de la linguistique de corpus moderne, nous appliquerons des tests de signifiance sur nos résultats. La sélection de ces tests demande une considération prudente des données envisagées. Comme il est usuel en linguistique de corpus, la nature des données analysées dans cette thèse est statistiquement exigeante et les données linguistiques comportent une série de particularités: premièrement, et contrairement aux sciences, les données linguistiques ont très rarement une distribution normale ${ }^{103}$, ce qui exclut bon nombre de tests statistiques courants (par exemple le test $t$ ). Deuxièmement, beaucoup de corpus contiennent des documents ou des textes de différents types et de différents auteurs. Dans T-zéro nous avons contrôlé le nombre d'énoncés par situation et par provenance des locuteurs, mais il y a toujours des individus qui parlent plus que d'autres ou qui utilisent d'avantage de négations.

Le premier défi peut être surmonté par la sélection d'un test qui ne demande pas de distribution normale, comme le test chi-carré de Pearson. Ce test nous aide à décider si les différences en termes de réalisation de ne que nous observons entre différents groupes de locuteurs ou entre diverses constructions syntaxiques sont dues à une coïncidence ou à une vraie influence du facteur en question. Supposons qu'un facteur quelconque (par exemple l'âge des locuteurs) n'ait aucune influence sur la réalisation de ne. Cette assomption est appelée hypothèse nulle $\left(\mathrm{H}_{0}\right)$. $\mathrm{Si} \mathrm{H}_{0}$ était pertinente, nous nous attendrions à une distribution à peu près similaire des réalisations et omissions de ne à travers les différents groupes testés. Par contre, dans la réalité, nous allons presque toujours observer des fluctuations aléatoires entre différents groupes qui apparaissent même si le facteur testé n'est pas vraiment pertinent. Le test

103 En théorie des probabilités, on assume qu'une variable aléatoire suit la loi normale gaussienne. Par exemple, le quotient intellectuel des êtres humains a une distribution normale: un grand nombre de personnes manifestent une intelligence moyenne et un nombre restreint de personnes possèdent une intelligence supérieure et inférieure à la norme. 
chi-carré compare donc les valeurs attendues selon l'hypothèse nulle avec les valeurs réellement observées. À l'aide du test, la probabilité ( $p$ ) que $\mathrm{H}_{0}$ soit correcte est calculée. Si $\mathrm{H}_{0}$ est correcte, les différences observées sont alors aléatoires. Par contre, si le facteur en question est pertinent, $\mathrm{H}_{0}$ doit être réfutée. Suivant la pratique commune en statistique, nous considérons comme statistiquement signifiante une différence entre plusieurs groupes s'il y a moins de $5 \%$ de probabilité que $\mathrm{H}_{0}$ soit valide (donc si $p<0.05$ ). ${ }^{104}$

La deuxième difficulté est dépassée en variant les tests appliqués. Dans un premier temps, la signifiance des facteurs individuels en fonction des données brutes est calculée à l'aide d'un calculateur chi-carré en ligne ${ }^{105}$. Ce test est valide si les valeurs attendues dépassent une fréquence de 5 occurrences, et c'est donc pour cette raison que certains groupes ont été regroupés (p.ex. les professeurs du lycée et de l'université) ou exclus (par exemple le type de phrase non identifiable) pour le calcul des tests.

Dans un second temps, nous calculons, pour les variables qui se sont montrées significatives, les moyennes des locuteurs par variante afin de les comparer. De cette façon, nous pouvons exclure qu'à l'intérieur des groupes les résultats soient biaisés par un déséquilibre entre les locuteurs qui produisent beaucoup de négations et ceux qui n'en produisent que peu. Cette deuxième étape n'est possible que lorsqu'il s'agit de variables sociodémographiques ou linguistiques récurrentes dans chaque énoncé (comme le type de sujet) et est exécutée (le cas échéant) à l'aide du logiciel statistique et de programmation $R$. Nous nous servons plus précisément des fonctions wilcox.test, kruskal.test et friedman.test, afin d'établir des différences significatives entre les moyennes des groupes de locuteurs par variable. Le test de Wilcox s'applique aux variables extralinguistiques binaires (par exemple le sexe des locuteurs) et le test de Kruskal-Wallis à celles qui comprennent plus

104 Nous tenons à noter que la conclusion inverse n'est pas admise: une valeur $p$ plus grande que 0.05 n'implique pas que le facteur en question n'est pas un effet significatif, mais seulement que nous ne pouvons pas prouver sa signifiance.

$105<$ http://www.physics.csbsju.edu/stats/contingency_NROW_NCOLUMN_form. html>. 
de deux variantes. Pour tester l'influence des variables linguistiques, nous utilisons le test de Friedman.

Dans l'analyse présenté en chapitre 4, nous noterons le type de test, les variantes comparées et la base du calcul (données brutes ou moyennes des locuteurs) dans les notes en bas de pages relatives aux valeurs $p$. Les valeurs $p$ sans aucune note dérivent des tests chi-carré de Pearson basés sur les résultats bruts des variantes notées dans le tableau.

\subsection{Résumé du chapitre 3}

Au cours de ce chapitre, nous avons décrit la conception et la genèse du corpus T-zéro (disponible sous $<$ http://server.linguistik.uzh.ch/cmeisner 2013-tzero», login: «TZ-Reader», mot de passe «TZero@ling!»), les données qu'il contient ainsi que les modalités de recherche qu'il offre. De plus, les protocoles méthodologiques pour l'enregistrement, la transcription et l'analyse de la variable $\pm n e$ et les tests statistiques utilisés dans l'analyse descriptive ont été expliqués en détail.

Malgré l'existence de nombreux corpus du français oral, c'est seulement à partir de la création de T-zéro qu'un corpus phonétiquement transcrit permet d'envisager le double contraste de la variation \pm ne sur l'axe diatopique (Suisse vs. France) et intrapersonnel (un locuteur dans deux situations).

Le corpus comprend environ 16150 mots produits par 72 locuteurs et locutrices de Suisse et de France. Une moitié des données linguistiques appartient au domaine de la distance communicative (les examens oraux) et l'autre à celui de l'immédiat communicatif (les conversations libres en groupe entre élèves, étudiants et chercheurs ainsi que les discussions qui ont eu lieu pendant les cours de l'assistante d'allemand). Les situations sont classées selon le modèle de Koch/Oesterreicher (2011).

Vu que la comparaison sur l'axe intrapersonnel est l'un des objectifs principaux de la création du corpus, sa composition sociodémographique 
s'explique par la sélection des situations enregistrées: les examens, qui offrent la possibilité d'observer, même dans la distance communicative, un langage dialogique et spontané, ont lieudans le milieu universitaire et scolaire, où plusieurs étudiants et élèves sont interrogés par un nombre restreint de professeurs ou chargés de cours. Par conséquent, la plupart des locuteurs en T-zéro est relativement jeune. En ce qui concerne la langue des locuteurs, la plupart indique le français comme seule langue maternelle, mais environ $20 \%$ des locuteurs sont bilingues ou se sont installés dans une zone francophone à l'âge adolescent.

T-zéro permet l'analyse et l'exportation des données linguistiques, sociodémographiques et situationnelles, accessibles à travers les masques constructions verbales (vert), personnes (rose) et enregistrements (jaune). De cette façon, la base de données offre même aux chercheurs inexpérimentés en linguistique de corpus la possibilité de lancer des requêtes (socio)linguistiques exigeantes.

Le protocole d'enregistrement suit la pratique internationalement reconnue, qui demande, par exemple, une déclaration de consentement des participants, et qui cherche à minimiser le paradoxe de l'observateur.

Le système de transcription de T-zéro est assez complexe: d'une part, nous suivons la pratique établie en linguistique de corpus de transcrire en orthographe standard adaptée, mais d'autre part, nous ajoutons une transcription phonétique en API. Ce type de transcription permet à la fois une lecture facile et une représentation fidèle des données linguistiques.

Pour l'analyse de la variable \pm ne envisagée dans ce travail, toutes les constructions négatives sont identifiées et exportées. Étant donné qu'il semble impossible de désambiguïser les cas où la réalisation de ne pourrait être confondue avec une liaison, comme dans on (n')a pas $v u$ [õnapavy], ceux-ci sont sont exclus après une brève discussion de la problématique. La signification statistique des résultats obtenus dans l'analyse de corpus sera évidemment testée statistiquement par les tests chi-carré appropriés. 


\section{L'analyse de corpus: $\pm n e$ dans le corpus T-zéro}

\subsection{Analyse globale: $\pm n e$ dans le corpus T-zéro}

La totalité des négations analysables ${ }^{106}$ dans le corpus se compose de 334 constructions négatives potentiellement bipartites: 18\% des négations contiennent la particule $n e$, tandis que la grande majorité des négations est exprimée uniquement à travers pas ou un autre élément négatif.

(114) \pm ne dans le corpus T-zéro

a. Pourcentages

La réalisation de ne dans T-zéro

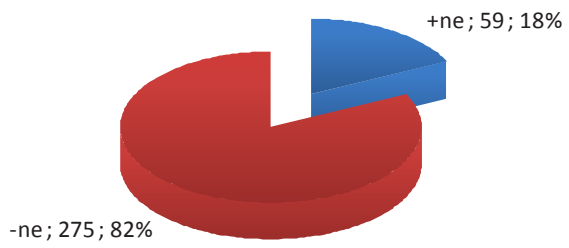

b. Données brutes

\begin{tabular}{ccccc}
\hline$+n e$ & $\%$ & $-n e$ & $\%$ & total \\
\hline 59 & $18 \%$ & 275 & $82 \%$ & 334 \\
\hline
\end{tabular}

106 Parmi les 2415 constructions verbales de T-zéro, nous avons trouvé un total de 360 verbes niés. Parmi ceux-ci, 26 constructions ont été exclues de l'analyse: dans deux cas, ne seul fonctionne comme négation; dans une construction coordonnée, non est employé comme négation verbale; et nous avons exclu 23 constructions négatives $(6 \%)$ dans lesquelles la réalisation éventuelle de $n e$ n'est pas perceptible, car elle coïncide avec une liaison, comme par exemple dans on (n') a pas [õnapa] (cf. section 3.8). Dû à la mise à jour continuelle de T-zéro, l'ensemble des résultats décrits ici ne peut pas être reproduit dans la version plus récente de T-zéro en ligne sous: $<$ http://server.linguistik.uzh.ch/cmeisner2013tzero>, login: «TZ-Reader», mot de passe «TZero@ling!». 
La nette prépondérance de l'omission de ne que nous observons en (114) confirme les résultats obtenus dans d'autres analyses de corpus portant sur le français phonique contemporain utilisé de manière spontanée ( $c f$. section 2.2.2).

\subsection{Analyse exploratrice: \pm ne selon le type de locuteur}

Les 334 occurrences négatives, qui seront analysées en détail par la suite, ont été produites par 54 locuteurs ${ }^{107}$ différents de Suisse et de France $^{108}$.

En nous basant sur une classification préliminaire et purement empirique extraite du corpus, nous distinguons trois groupes de locuteurs:

(115) La classification empirique des trois types de locuteurs et leurs caractéristiques

\begin{tabular}{|lll|}
\hline Type A & montre une variation $\pm n e$ & Ce type représente le francophone européen typique. \\
Type B & $\begin{array}{l}\text { montre uniquement } \\
\text { l'absence de } n e\end{array}$ & $\begin{array}{l}\text { Une généralisation de la négation monopartite } \\
\text { au niveau de la compétence linguistique est } \\
\text { éventuellement possible. }\end{array}$ \\
Type C & $\begin{array}{l}\text { montre uniquement la } \\
\text { présence de } n e\end{array}$ & $\begin{array}{l}\text { Il s'agit d'une pure coïncidence empirique et la } \\
\text { généralisation de la négation bipartite au niveau de la } \\
\text { compétence linguistique semble exclue. }\end{array}$ \\
\hline
\end{tabular}

Le type A manifeste une variation $\pm n e$, le type $\mathrm{B}$ montre uniquement l'absence de ne et le type $\mathrm{C}$ indique une présence absolue de $n e$.

107 Davantage d'informations sur les locuteurs sont disponibles dans T-zéro, dans le masque Personnes (cherchez le numéro d'identification respectif).

108 Pendant l'enregistrement, les locuteurs produisent entre 1 et 34 négations par personne. Ce nombre assez restreint de négations produites par personne est dû au fait que les intervalles enregistrés et transcrits par locuteur sont courts (entre deux et cinq minutes). De plus, comme il s'agit d'une production spontanée, la chercheuse n'a pas incité la production de négations dans le discours ( $c f$. section 3.6 pour le protocole d'enregistrement). 
La vue d'ensemble des occurrences négatives produites par les trois types de locuteurs en (116) et (117) montre que la majorité des négations dans le corpus est produite par les locuteurs du type A, suivi du type $\mathrm{B}$, et que seul un nombre restreint d'énoncés négatifs provient des locuteurs du type $\mathrm{C}$.

(116) La variation \pm ne par type de locuteur

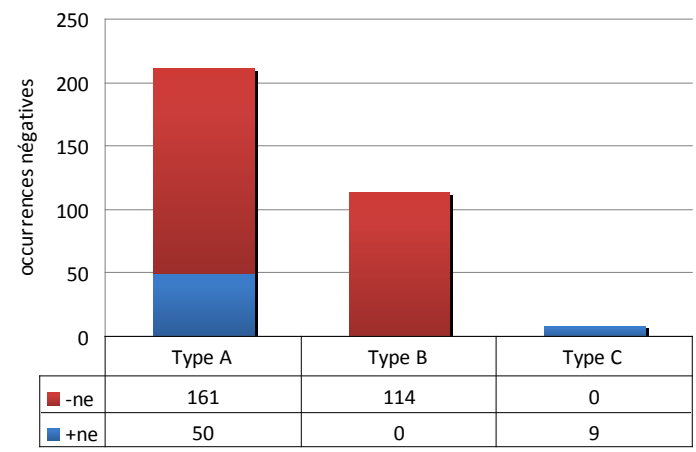

(117) La distribution des occurrences négatives en fonction du type de locuteur Les occurrences négatives produites par les types de locuteurs (total :

54 locuteurs produisent 334 négations)

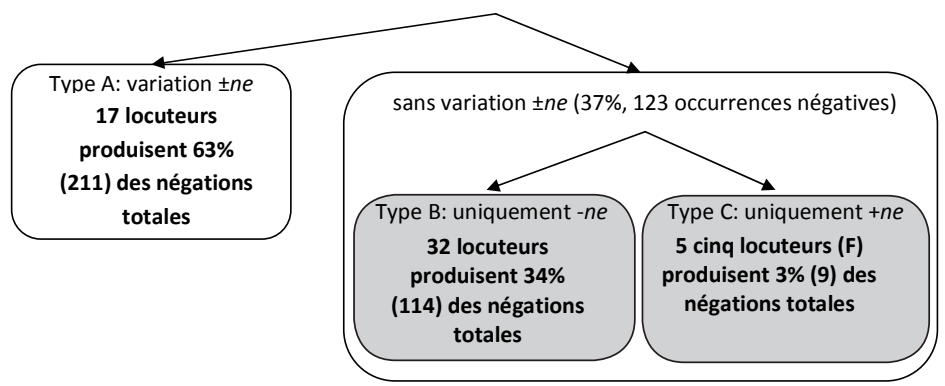

Le type A produit $63 \%$ des négations de phrase dans le corpus, tandis que les $37 \%$ d'occurrences négatives restantes sont produits par des locuteurs qui ne montrent aucune variation dans le corpus. Dans ce dernier groupe, le type B, qui produit uniquement la négation monopartite, est clairement dominant. Les 32 locuteurs du type B produisent $34 \%$ des 
négations dans notre corpus, et seulement 3\% des négations totales sont produits par 5 locuteurs de France qui n'utilisent que la négation bipartite.

L'analyse qui suit expliquera que le type le plus dominant dans le corpus, à savoir le type A, représente également au niveau de la compétence linguistique le francophone européen typique ( $c f$. section 4.2.1). Quant aux types B et C, qui ne montrent qu'une variante dans le corpus, ils sont plus difficiles à interpréter.

Le type B (analysé en détail en section 4.2.2) produit un nombre considérable de négations dans le corpus (34\%), et même si beaucoup de ces occurrences sans ne sont explicables par des facteurs linguistiques (comme par exemple un sujet clitique léger, $c f$. section 2.2.5.1), ceci ne vaut pas pour toutes les occurrences. Il semble donc possible que quelques individus appartenant au type B aient (déjà) généralisé la négation monopartite dans leurs grammaires internes, ce qui signifie qu'au niveau de la compétence, l'existence du type B n'est pas exclue. Comme l'analyse du type B le montrera, les données de corpus ne suffisent pas pour prouver l'existence d'un tel type au niveau de la compétence, un fait éventuellement démontrable à l'aide des tests d'intuition (cf. Coveney 1998, Meisner 2013).

Si l'on pouvait montrer à l'aide des tests d'intuition que certains locuteurs ont effectivement généralisé l'absence de ne, ceci signifierait qu'ils auraient développé une 'nouvelle compétence' et devraient, par conséquent, être exclus de futures analyses de la variation $\pm n e$, tout simplement parce qu'ils ne manifestent pas de variation que l'on pourrait étudier. Néanmoins, vu que nous ne pouvons pas prouver empiriquement l'existence du type B, les données des locuteurs en question ne seront pas exclues de l'analyse.

En ce qui concerne le type C, il n'y a aucun motif d'assumer l'existence de francophones compétents qui appartiennent à ce groupe, décrit en section 4.2.3. Ces individus sont très rares dans le corpus et ils ne produisent que 3\% des négations totales. Ces occurrences rares sont toutes explicables en raison des influences linguistiques (comme par exemple un sujet lourd, $c f$. section 2.2.5.2), ce qui fait que la raison la plus probable pour l'absence d'une variation \pm ne dans ces cas-là est le nombre restreint de négations produites par ces individus. Le fait que 
les locuteurs du type $\mathrm{C}$ montrent uniquement une variante pendant l'enregistrement est donc probablement une pure coïncidence empirique.

Afin d'analyser plus profondément les différents types de locuteurs, nous considérerons, dans les sections suivantes, leurs caractéristiques sociodémographiques et quelques particularités linguistiques des négations qu'ils produisent.

\subsubsection{Type A: les locuteurs qui montrent une variation \pm ne}

Les 17 locuteurs du type A produisent chacun entre 2 et 34 occurrences négatives dans le corpus, ils constituent environ un tiers des locuteurs enregistrés dans notre corpus et produisent un total de 211 négations de phrase, ce qui correspond à $63 \%$ des négations contenues dans le corpus. Les pourcentages de $+n e$ de ce groupe se situent entre $6 \%+n e$, produit par une doctorante neuchâteloise, et $75 \%+n e$, produit par un professeur de lycée en région parisienne.

Considérons à présent les caractéristiques extralinguistiques qui unissent les locuteurs du type $\mathrm{A}^{109}$. Tout d'abord, ce groupe comprend aussi bien des locuteurs et des locutrices suisses que français de 14 à 64 ans. Quant à la langue, il s'agit uniquement de locuteurs monolingues, majoritairement des francophones natifs. Seuls deux locuteurs L2 avancés de Suisse romande ayant comme langue maternelle le suisse allemand figurent également dans ce groupe. Au niveau professionnel et éducatif, l'ensemble des secteurs professionnels ${ }^{110}$ qui ont été considérés dans cette étude apparaît dans le type A: il s'agit d'élèves, d'étudiants, de docteurs et chercheurs à l'université ainsi que des professeurs au lycée et à l'université.

Dans l'ensemble, les locuteurs du type A sont donc indéterminables socio-démographiquement: ce sont des hommes et des femmes, jeunes

109 Dans l'annexe VI figure une liste des locuteurs du type A et de leurs caractéristiques démographiques en détail.

110 Nous rappelons que, dans le but de comparer des situations d'examens oraux avec des conversations détendues, les enregistrements ont était faits dans le milieu universitaire et scolaire, ce qui restreint la variation sociale entre les individus ( $c f$. section 3.2 pour une description sociodémographique du corpus). 
et âgés, natifs ou L2 qui proviennent de différentes régions ou secteurs professionnels. Nous pouvons donc conclure que le type A, qui produit aussi bien des négations bipartites que monopartites, peut être considéré comme le 'prototype' du francophone européen.

\subsubsection{Type B: négation monopartite uniquement}

Même si les locuteurs du type A produisent la plupart des négations dans le corpus, un nombre considérable de négations, plus précisément un tiers des négations au total, est produit par des locuteurs du type B, qui ne manifestent jamais de négations bipartites dans le corpus.

Pour le type B, le nombre de négations produites est presque proportionnellement inverse au nombre de locuteurs, c'est-à-dire que ce groupe se compose d'un grand nombre de locuteurs qui produisent chacun un nombre restreint de négations. Seuls sept locuteurs du type B produisent plus de quatre négations, alors que la plupart des locuteurs dans ce groupe produit uniquement une ou deux négations. L'on peut alors se demander si l'existence du type B est due au grand nombre de locuteurs qui produisent assez peu de négations.

En effet, plus de la moitié des locuteurs suisses et français empiriquement attribués au type $\mathrm{B}$ ne produisent qu'une ou deux négations (cf. annexe VII pour une liste détaillée des locuteurs du type B). Le nombre restreint de négations produites est donc très probablement la raison principale pour laquelle nous n'observons aucune variation $\pm n e$ dans ce groupe de locuteurs. La plupart de ces cas s'explique par la courte durée de l'enregistrement ou par le nombre restreint de négations totales produites par locuteur.

Toutefois, dans le cas de huit locutrices ${ }^{111}$ suisses, qui ont été enregistrées aussi bien dans les situations d'examen que de l'immédiat, sans

111 Le fait que ce ne sont que des femmes qui omettent systématiquement ne dans les deux situations est probablement dû à leur surreprésentation dans notre corpus ( $c f$. section 3.2). Toutefois, la comparaison avec davantage d'hommes dans les mêmes situations serait une piste de recherche future intéressante. 
y manifester la variante $+n e$, l'absence constante de ne est un comportement cohérent qui pourrait faire partie de leurs grammaires individuelles. Ici, l'absence de ne semble indépendante non seulement de la situation de communication, mais également du contexte linguistique.

À titre d'exemple, nous présentons en (118) les données de l'étudiante bilingue Lo_CH_F_1004 qui, sur 22 négations, ne réalise aucun $n e$, et ceci malgré le fait que la majeure partie de ses énoncés négatifs est produite lors d'un examen oral.

(118) L'absence de ne dans tous les contextes? Les énoncés négatifs de CH_F_1004 $4^{112}$

\begin{tabular}{|c|c|c|c|c|c|}
\hline ID & exemple & Situation & Sujet & Temps & Phrase \\
\hline 0021 & qu'il le maîtrise pas si bien que ça & dist & cl.lé & ind. prés. & sub \\
\hline 0023 & $\begin{array}{l}\text { Arius à mon sens c'est sûrement pas un } \\
\text { savant }\end{array}$ & dist & dou & ind. prés. & prin \\
\hline 0025 & $\begin{array}{l}\text { je l'apparentrais ni à un savant peut-être } \\
\text { pas non plus à un homme d'église }\end{array}$ & dist & cl.lé & $\begin{array}{l}\text { cond. } \\
\text { prés. }\end{array}$ & prin \\
\hline 0029 & c'était peut-être pas ça votre question & dist & cl.lé & ind. prés. & prin \\
\hline 0031 & j'ai pas réfléchi & dist & cl.lé & ind. $\mathrm{pc}$ & prin \\
\hline 0035 & $\begin{array}{l}\text { ben c'est pas quelqu'un que } \\
\text { j'apparentrais à quelqu'un de savant }\end{array}$ & dist & cl.lé & ind. prés. & prin \\
\hline 0037 & je sais pas & dist & cl.lé & ind. prés. & prin \\
\hline 0039 & il me semble pas avoir croisé d'Arius & dist & cl.lé & ind. prés. & prin \\
\hline 0041 & je savais pas si c'était & dist & cl.lé & ind. imp. & prin \\
\hline 0049 & $\begin{array}{l}\text { le bouleversement vocalique je pense pas } \\
\text { le citer }\end{array}$ & dist & cl.lé & ind. prés. & sub \\
\hline 0051 & c'est pas le même phénomène & dist & cl.lé & ind. prés. & prin \\
\hline 0053 & je sais pas & dist & cl.lé & ind. prés. & prin \\
\hline 0061 & je sais plus & dist & cl.lé & ind. prés. & prin \\
\hline 0065 & je sais plus & dist & cl.lé & ind. prés. & prin \\
\hline
\end{tabular}

112 dist $=$ distance communicative, cl.lé $=$ clitique léger, pron.lou $=$ pronom lourd, lex $=$ sujet lexical, ind. prés. $=$ indicatif présent, cond. prés. $=$ conditionnel présent, ind. fut. périph. $=$ indicatif futur périphrastique, ind. $p c=$ indicatif $=$ passé composé, prin $=$ phrase principale, $s u b=$ phrase subordonnée. 


\begin{tabular}{|llllll|}
\hline 0161 & on va plus en avoir que des courtes & dist & cl.lé & $\begin{array}{l}\text { ind. fut. prin } \\
\text { périph. }\end{array}$ \\
0223 qui est pas un terme péjoratif & dist & pron.lou & ind. prés. sub \\
0243 & $\begin{array}{l}\text { le latin archä̈que était que du latin } \\
\text { classique }\end{array}$ & dist & lex & ind. prés. prin \\
0295 y a pas de différences parce que c'est & dist & pron.lou & ind. prés. prin \\
$0319 \begin{array}{l}\text { je sais plus si c'est diamésique ou } \\
\text { diamérique }\end{array}$ & dist & cl.lé & ind. prés. prin \\
0715 je sais pas si c'est allé & imméd & cl.lé & ind. prés. prin \\
0789 où je connais pas la langue & imméd & cl.lé & ind. prés. sub \\
0801 que je le sais pas l'anglais & imméd & cl.lé & ind. prés. sub \\
\hline
\end{tabular}

Bien que la locutrice $\mathrm{CH}_{-} \mathrm{F} \_1004$ produise un nombre considérable de négations dans différents contextes linguistiques, le facteur linguistique le plus pertinent, à savoir le type de sujet, varie peu. Dans la majorité des cas, un clitique sujet léger (abrégé dans le tableau par cl.lé) est employé, ce qui incite normalement à l'omission de ne, comme les analyses de corpus résumées dans la section 2.2.5.1 l'ont montré. Seul l'énoncé 0243 comporte le DP lourd le latin archaïque dans la position de sujet, ce qui normalement déclencherait, du moins statistiquement, l'emploi de la particule ne. La rareté des sujets lexicaux dans le langage spontané est parfaitement naturelle ( $c f$. section 5.3), mais nous nous demandons si un seul énoncé de ce type est suffisant pour argumenter en faveur d'une généralisation de la négation monopartite dans la grammaire individuelle de CH_F_1004.

Afin d'approfondir cette question, considérons maintenant l'ensemble des 56 énoncés négatifs sans ne produits par CH_F_1004 et les autres sept locutrices suisses en question, qui figurent sous (119). Tout d'abord, nous observons que la distribution des occurrences négatives à travers les situations de communication est très équilibrée: il y a autant de négations sans ne dans la distance communicative que dans l'immédiat communicatif. Une telle 'négligence' du degré de formalité de la situation semble être un phénomène typiquement suisse (cf. aussi Fonseca-Greber 2007), qui s'oppose au comportement typique des Français. 
(119) Les caractéristiques des énoncés négatifs sans ne de 8 locutrices suisses

\begin{tabular}{|c|c|c|}
\hline Paramètre & Valeurs & No. d'occurrences \\
\hline \multirow[t]{2}{*}{ Situation } & distance communicative & 29 \\
\hline & immédiat communicatif & 27 \\
\hline \multirow[t]{5}{*}{ Sujet } & cl.lé & 44 \\
\hline & pron.lou & 5 \\
\hline & lexical & 1 \\
\hline & redoublé & 6 \\
\hline & & 56 \\
\hline \multirow[t]{9}{*}{ Pronom sujet } & $i l$ & 2 \\
\hline & ce & 6 \\
\hline & je & 31 \\
\hline & il impersonnel & 3 \\
\hline & on & 3 \\
\hline & $t u$ & 2 \\
\hline & $c ̧ a$ & 3 \\
\hline & elle & 2 \\
\hline & $q u i$ & 3 \\
\hline \multirow[t]{2}{*}{ Phrase } & principale & 44 \\
\hline & subordonnée & 12 \\
\hline \multirow[t]{6}{*}{ Temps } & indicatif présent & 39 \\
\hline & indicatif imparfait & 9 \\
\hline & indicatif passé composé & 2 \\
\hline & indicatif futur périph. & 3 \\
\hline & conditionnel présent & 2 \\
\hline & conditionnel passé & 1 \\
\hline
\end{tabular}

Nous observons à l'aide du tableau (119) qu'également au niveau linguistique l'absence de ne semble assez indépendante du contexte: les locutrices omettent la particule de négation dans les subordonnées et les principales, avec un verbe au présent, au passé composé et au futur périphrastique de l'indicatif, mais également au conditionnel.

Or, au niveau du sujet, l'occurrence du sujet lexical produit par CH_F_1004, déjà cité en (118), reste un cas unique, ce qui rend difficile l'évaluation définitive des données en (118) et (119). Nous notons tout 
de même que parmi les pronoms sujet se trouvent non seulement des clitiques légers, mais également des pronoms lourds (pron.lou) comme qui et ça, qui pourraient favoriser l'emploi de ne, même si à un moindre degré que les sujets lexicaux.

Dans l'ensemble, nous ne pouvons pas prouver que le type B existe réellement au niveau de la compétence linguistique et que cette impression n'est pas due à une coïncidence empirique. Afin d'établir des connaissances solides, il faudrait à l'avenir varier les méthodes d'étude: par exemple, les locutrices susceptibles d'appartenir au type B pourraient être confrontées, lors de tests d'acceptabilité ciblés, à des négations monopartites contenant divers types de sujet ( $c f$. Coveney 1998, Meisner 2013). Les tests d'acceptabilité effectués à ce sujet par Meisner (2013: 126) montrent clairement que les élèves de la région parisienne (qui sont en partie également enregistrés dans le présent corpus) distinguent clairement les négations monopartites avec des sujets DP lourds de celles avec des sujets clitiques légers: les dernières leur semblent constamment plus acceptables que les premières (indépendamment de la situation de communication).

Par conséquent, si ces huit locutrices suisses susceptibles d'avoir généralisé la variante sans ne acceptaient l'absence de ne de la même façon dans des exemples contenant un sujet lourd qu'un clitique léger, il serait effectivement très probable que la négation monopartite soit réellement fixée dans leurs grammaires individuelles (du moins pour la production langagière phonique).

\subsubsection{Type C: négation bipartite uniquement}

Un nombre très restreint de neuf négations, ce qui correspond à 3\% des négations analysées, a été produit par 5 élèves d'Île-de-France qui ne produisent jamais la négation sans ne ( $c f$. annexe VIII pour une liste de ces locuteurs). Parmi eux, quatre ne produisent qu'une seule négation et ne peuvent donc montrer aucune variation.

Seul un lycéen français, enregistré uniquement dans une situation de distance communicative et qui produit cinq négations bipartites, au- 
rait la possibilité de montrer les deux variantes de la négation. L'on se demande donc si la négation bipartite qu'il emploie constamment dans notre corpus fait partie d'une stratégie communicative appliquée pendant l'examen oral ou si elle est éventuellement un trait idiosyncratique de la grammaire de ce jeune homme français.

L'extrait de notre base de données en (120) montre qu'il ne s'agit ni d'une stratégie communicative ni d'une idiosyncrasie grammaticale. $\mathrm{Au}$ contraire, ce sont très probablement les caractéristiques linguistiques des énoncés qui incitent à la réalisation de $n e$.

(120) Les énoncés négatifs produits par Lo_FR_M_1056

\begin{tabular}{|llllll|}
\hline ID & Exemple & Situation & Sujet & Temps & Phrase \\
\hline 0330 & $\begin{array}{l}\text { que son væu de sagesse ne lui a pas apporté } \\
\text { grand-chose }\end{array}$ & dist & lex & ind.pc & sub \\
$0342 \begin{array}{l}\text { et que son vou de sagesse ne lui a pas été } \\
\text { profitable }\end{array}$ & dist & lex & ind.pc & sub \\
0356 & l'erreur ne vient pas que de lui & dist & lex & ind. prés. & sub \\
1006 & $\begin{array}{l}\text { alors qu'il avait dit qu'il n'aura pas de } \\
\text { problèmes avec ses amis }\end{array}$ & dist & cl.lé & ind. fs & sub \\
1058 & que l'homme n'est pas perfectible & dist & lex & ind. prés. sub \\
\hline
\end{tabular}

Parmi les cinq énoncés dans le tableau (120), quatre contiennent des sujets DP lourds, ce qui favorise fortement la réalisation de la particule ne (cf. section 2.2.5.1), et la seule construction avec un sujet pronominal contient un verbe au futur simple en attaque vocalique, ce qui semble avoir un effet similaire ( $c f$. les sections 2.2.5.5 et 2.2.5.7). De plus, tous les énoncés négatifs produits par Lo_FR_M_1056 apparaissent dans des constructions subordonnées, qui sont également connues comme l'un des contextes déclencheurs de la négation bipartite.

Nous pouvons donc conclure que ce sont avant tout ces caractéristiques linguistiques particulières, observées chez le locuteur Lo_ FR_M_1056, qui incitent à l'emploi de ne (cf. sous-chapitre 2.2.5 pour une discussion détaillée des facteurs linguistiques qui influencent la variation $\pm n e$ ). Il n'y a donc aucune raison d'assumer que la réalisation constante de ne serait un trait de sa grammaire. 


\subsection{Analyse descriptive de l'influence des facteurs extralinguistiques}

Dans les sections précédentes, nous avons choisi une approche pilotée par les données, c'est-à-dire que nous avons abordé le corpus à travers les données linguistiques ( $c f$. section 3.5 pour l'accès aux données linguistiques de T-zéro) afin de former des groupes de locuteurs en fonction de leur production de ne, à savoir les types A, B et C.

Dans les sections qui suivent, nous adapterons la perspective pilotée par la théorie sociolinguistique qui est typiquement employée dans les études de corpus quant à la variable $\pm n e$ ( $c f$. entre autres Armstrong/ Smith 2002, Ashby 1976, 1981, 2001, Coveney ${ }^{2} 2002$, Hansen/Malderez 2004). Ceci signifie que nous considérons les faits linguistiques dans notre corpus en partant des facteurs extralinguistiques qui ont joué un rôle pour la réalisation de la variable dans d'autres analyses ( $c f$. section 2.2.4 pour un résumé de l'influence des facteurs extralinguistiques sur \pm ne et section 3.4 pour l'accès aux données extralinguistiques dans la base de données T-zéro).

\subsubsection{La diatopie}

Le tableau (121) montre la réalisation de \pm ne en fonction de la provenance géographique des locuteurs. Nous comparons les données de Suisse, enregistrées à Neuchâtel, avec celles de France, enregistrées en Île-de-France ( $c f$. section 3.2).

(121) La réalisation de $\pm n e$ en Suisse et en France

\begin{tabular}{|lcccccc|}
\hline & $+n e$ & $\%$ & $-n e$ & $\%$ & Total & $\chi^{2}$ \\
\hline CH & 15 & $10 \%$ & 136 & $90 \%$ & 151 & $p=0,398^{113}$ \\
FR & 44 & $24 \%$ & 139 & $76 \%$ & 183 & \\
Total & 59 & $18 \%$ & 275 & $82 \%$ & 334 & \\
\hline
\end{tabular}

113 Test de Wilcox chi-carré appliqué aux moyennes des locuteurs. 
Dans notre corpus, la fréquence de la réalisation de ne varie considérablement en fonction de la provenance géographique. Il saute à l'œil que la réalisation de ne en Suisse est avec 10\% +ne nettement plus basse que les $24 \%$ + ne en Île-de-France.

Comme Fonseca-Greber (2007) l'a déjà remarqué, les francophones de Suisse manifestent, du moins dans l'immédiat communicatif, des taux très restreints de $n e$ :

(122) $n e$ is used in only $2.5 \%$ of the total negative utterances produced (n. $=1,982)$. In other words, $n e$ is all but gone from Conversational Swiss French. To my knowledge, this result represents the lowest figure attested so far for $n e$ use in studies that include educated adult middle-class speakers of European French. (Fonseca-Greber 2007: 256)

Les résultats de Fonseca-Greber (2007) pour la Suisse romande semblent confirmés par les taux restreints de + ne dans notre corpus. Comme nous l'avons vu en section 4.2.2, quelques locutrices suisses sont mêmes susceptibles d'avoir généralisé l'absence de ne indépendamment de la situation de communication ou du contexte linguistique.

Toutefois, nous constatons que le ne n'est pas inexistant dans le français de Suisse. Dans notre corpus, la réalisation de ne en Suisse est clairement plus élevée que celle trouvée par Fonseca-Greber (2007) et, même si elle reste nettement plus basse qu'en France, la différence entre les deux pays n'est pas significative. Autrement dit, les résultats dans le tableau (121) pourraient être dus à une coïncidence ( $c f$. section 3.9 pour une description détaillée des tests statistiques employés dans ce chapitre).

Il s'agira donc, dans ce qui suit, d'examiner avec précaution ces $10 \%+n e$ trouvés en Suisse et de se demander pourquoi ces cas échappent à la tendance générale d'absence du ne. 


\subsubsection{L’âge des locuteurs}

La comparaison de la réalisation de ne en fonction de l'âge des individus sous (123) révèle une tendance intéressante: jusqu'à l'âge de 30 ans environ, les locuteurs omettent plus de la moitié des ne possibles. Or, à partir de 40 ans, les personnes produisent plus de négations bipartites que monopartites. Un tournant concernant la réalisation de ne semble donc se manifester entre 30 et 40 ans.

(123) La réalisation de ne en fonction de l'âge des locuteurs_trices

\begin{tabular}{|cccccccccccccc|}
\hline & $14-18$ & $\%$ & $19-24$ & $\%$ & $25-34$ & $\%$ & $35-44$ & $\%$ & $45-54$ & $\%$ & $55-64$ & $\%$ & $\chi^{2}$ \\
\hline$-n e$ & 135 & 79 & 76 & 95 & 59 & 84 & 1 & 50 & 1 & 33 & 3 & 33 & $p=0.1481^{114}$ \\
$+n e$ & 35 & 21 & 4 & 5 & 11 & 16 & 1 & 50 & 2 & 67 & 6 & 67 & \\
\hline
\end{tabular}

Nous observons à l'âge de 14-18 ans un taux de réalisation de $21 \%+n e$, qui est légèrement supérieur à la moyenne du corpus de $18 \%$, cependant à 19-24 ans la réalisation de ne diminue à 5\% et atteint son niveau plancher. À l'âge de 25-34 ans, la production moyenne des négations bipartites recommence à monter à $16 \%$, et entre 35 et 44 ans, la relation entre les négations sans et avec ne se stabilise à $50 \%$. Les locuteurs de 45-54 et de 55-46 ans produisent en moyenne 67\% des négations avec la particule ne. Toutefois, la variation entre les différents groupes d'âge dans notre corpus n'est pas statistiquement significative et pourrait donc être due à une coïncidence.

\subsubsection{La diastratie: la profession et la formation des locuteurs}

Au niveau de la profession et de la formation des locuteurs, nous observons les tendances suivantes quant à la réalisation de $n e$ :

114 Test de Kruskal-Wallis chi-carré appliqué sur les moyennes des locuteurs. 
(124) La réalisation de ne en fonction de la profession/formation des locuteurs trices

\begin{tabular}{|lrrrrrrrrrrrrc|}
\hline & élève & $\%$ & étud & $\%$ & prof lycée & $\%$ & doc & $\%$ & prof uni & $\%$ & cherch & $\%$ & $\chi^{2}$ \\
\hline$+n e$ & 35 & 21 & 4 & 5 & 9 & 69 & 4 & 7 & 6 & 67 & 1 & 20 & $0,076^{115}$ \\
$-n e$ & 135 & 79 & 76 & 95 & 4 & 31 & 53 & 93 & 3 & 33 & 4 & 80 & \\
\hline
\end{tabular}

Les deux premiers groupes, c'est-à-dire les élèves français et les étudiants suisses, coïncident avec les groupes d'âge de 14-18 et 19-24 (cf. section 3.2). Pour les groupes plus âgés, le tableau (124) nous offre des détails intéressants: la production de ne des doctorants suisses est presque aussi basse que celle des étudiants $(7 \%+n e)$. Les chercheurs que nous avons testés produisent $20 \%$ des ne, ce qui correspond approximativement à la production des élèves français.

Il y a une coupure claire et nette entre les professeurs, que nous n'avons pu enregistrer que lors des examens, et les autres groupes. Les professeurs d'école français produisent $69 \%$ des ne possibles et les professeurs suisses en réalisent $67 \%$. Dans l'ensemble, nous observons une production de ne assez basse chez les élèves et les étudiants et considérablement élevée chez les professeurs. Le fait que cette différence n'est pas statistiquement significative pourrait être dû au déséquilibre entre les professions, en effet, dans notre corpus, les élèves et les étudiants sont surreprésentés ( $c f$. section 3.2).

\subsubsection{Locuteurs bilingues et monolingues}

Le dernier facteur interpersonnel que nous avons testé est la langue maternelle des locuteurs. Bien que tous les locuteurs aient une excellente performance en français, il y a des différences quant à leur statut bi- ou monolingue et L1 ou L2. Dans le diagramme (125) figure la réalisation de ne des 43 locuteurs français monolingues, des 8 locuteurs bilingues et celle des 3 locuteurs L2 qui habitent une zone francophone depuis des années.

115 Test de Kruskal-Wallis chi-carré appliqué sur les moyennes des locuteurs. 
(125) La réalisation de ne selon la langue maternelle

a. Pourcentages

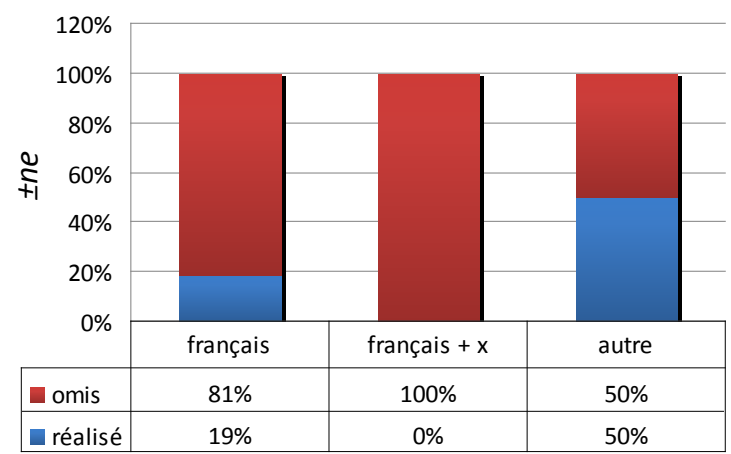

b. Données brutes

\begin{tabular}{|lrrrrrr|}
\hline & $+n e$ & \multicolumn{1}{c}{$\%$} & \multicolumn{1}{c}{$-n e$} & \multicolumn{1}{c}{$\%$} & \multicolumn{1}{c|}{ total } & \multicolumn{1}{c|}{$\chi^{2}$} \\
\hline français L1 & 51 & $19 \%$ & 223 & $81 \%$ & 274 & $p=0,038^{116}$ \\
français $+\mathrm{x}$ & 0 & $0 \%$ & 44 & $100 \%$ & 44 & \\
autre & 8 & $50 \%$ & 8 & $50 \%$ & 16 & \\
total & 59 & $18 \%$ & 275 & $82 \%$ & 334 & \\
\hline
\end{tabular}

Les locuteurs monolingues, qui n'indiquent que le français comme langue maternelle, ont un taux moyen de $22 \%+n e$. Pour les locuteurs qui ont appris le français comme L2, la moyenne est légèrement plus élevée $(33 \%+n e)$. Quant à la moyenne des locuteurs bilingues, elle constitue la valeur la plus frappante dans ce diagramme: ces locuteurs ne réalisent jamais la particule de négation dans notre corpus.

Nous tenons à signaler que la tendance qui se cristallise ici nous semble digne d'une réflexion approfondie. En effet, l'on peut se demander si les locuteurs généralisent les systèmes de négation de leurs deuxièmes langues maternelles sur le français. Comme deuxième langue maternelle, trois individus indiquent l'arabe, deux indiquent respectivement le turc et le suisse-allemand et une locutrice parle l'anglais en plus du français.

116 Test de Kruskal-Wallis chi-carré appliqué sur les moyennes des locuteurs. 
Effectivement, mis à part l'arabe, ces langues se servent des morphèmes postverbaux (comme pas) pour exprimer la négation. De plus, vu que la différence entre les différentes langues maternelles est statistiquement significative, nous avons décidé d'approfondir l'hypothèse du contact linguistique dans l'un des paragraphes de la discussion ( $c f$. section 5.1.5), parallèlement à la discussion des approches établies dans la littérature scientifique, qui seront discutées dans les sections 5.1.1 à 5.1.4.

\subsubsection{La variation situationnelle}

Dans les analyses de $\pm n e$, la situation de communication apparaît comme un facteur puissant mais rarement testé à cause des difficultés méthodologiques ( $c f$. section 2.4.4.4). Pour cette raison, nous accordons une attention particulière à ce facteur.

Dans notre corpus, qui cible la variation intrapersonnelle ( $c f$. section 3.3), les taux de réalisation de ne atteignent $35 \%$ des cas dans les énoncés enregistrés pendant les examens oraux ( $c f$. section 3.3.1). La réalisation de ne dans la distance communicative est donc significativement plus élevée que lors des conversations et discussions, classées comme situations de l'immédiat communicatif ( $c f$. section 3.3.2), où la réalisation de la particule est plutôt marginale avec $5 \%+n e$. Toutefois, dans l'ensemble, le nombre de négations bipartites reste, même dans la distance communicative, inférieur à la moitié des négations totales.

(126) La réalisation de $n e$ en fonction de la situation de communication

\begin{tabular}{|lrrrrrr|}
\hline & $+n e$ & \multicolumn{1}{c}{$\%$} & $-n e$ & $\%$ & Total & $\chi^{2}$ \\
\hline Distance communicative & 50 & $35 \%$ & 91 & $65 \%$ & 141 & $p=0,025^{117}$ \\
Immédiat communicatif & 9 & $5 \%$ & 184 & $95 \%$ & 193 & \\
Moyenne du corpus & 59 & $100 \%$ & 275 & $100 \%$ & 334 & \\
\hline
\end{tabular}

117 Test de Friedman appliqué sur les moyennes des locuteurs par situation de communication. 
La réalisation de ne est significativement inférieure dans l'immédiat que dans la distance communicative, ce qui ne peut donc pas être une différence aléatoire.

Néanmoins, contrairement à l'affirmation de Koch/Oesterreicher (22011: 172), qui soutiennent que la réalisation de ne serait absolue dans les situations de distance communicative, nous avons vu que les taux de ne n'atteignent pas, même dans la distance communicative, la moitié des cas.

Voyons maintenant s'il y a des différences entre les deux pays, la France et la Suisse romande, quant à l'influence de la situation de communication. Dans le diagramme (127)a et dans le tableau (127)b figurent les taux de réalisation et omission de ne par situation de communication en Suisse (colonne de gauche) et en France (colonne de droite).

(127) La réalisation de ne selon la situation de communication en $\mathrm{CH}$ et $\mathrm{F}$

a. Pourcentages

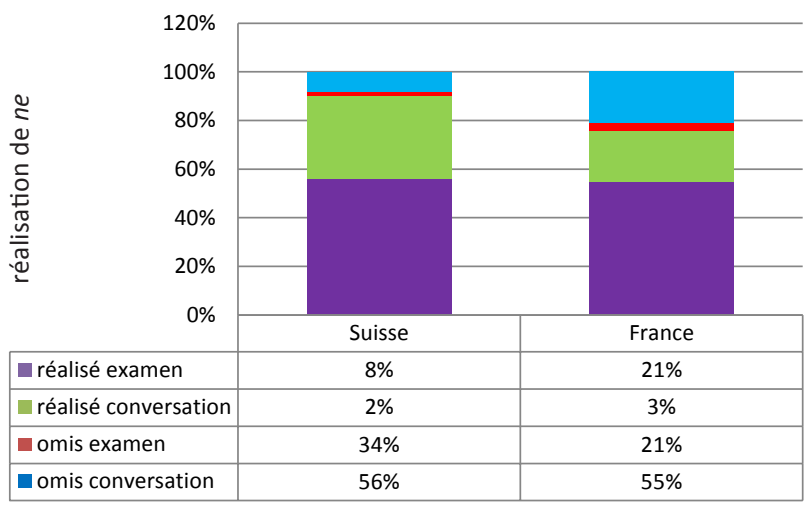

b. Données brutes

\begin{tabular}{|lccccc|}
\hline & $+n e$ examen & $+n e$ conversation & $-n e$ examen & $-n e$ conversation & $\chi^{2}$ \\
\hline CH & 12 & 3 & 52 & 84 & $p=0,002$ \\
FR & 38 & 6 & 39 & 100 & \\
Total & 50 & 9 & 91 & 184 & \\
\hline
\end{tabular}


Dans le diagramme (127)a, chaque colonne représente l'ensemble des négations produites par pays. Les différentes couleurs indiquent si ne a été réalisé ou non et dans quelle situation de communication: les omissions de ne figurent en violet s'il s'agit d'une situation de l'immédiat et en vert si elles ont lieu pendant un examen. Les parties bleues représentent les ne réalisés pendant un examen et les parties rouges les occurrences produites pendant une conversation.

Le diagramme (127)a montre que, dans le domaine de l'immédiat, la (non-)réalisation de $n e$ est presque égale dans les deux pays: ceci se voit dans le fait que les parties violettes (omis conversations) et rouges (réalisé conversations) sont quasiment identiques dans les deux colonnes.

En revanche, le comportement des locuteurs en situation d'examen diffère. Les Suisses, représentés par la colonne de gauche, y omettent la majorité des ne, comme la grande partie verte (omis examens) dans la colonne suisse le montre, et ne réalisent la particule ne qu'occasionnellement, comme la petite partie bleue (réalisé examen) l'indique.

Par contre, les Français, représentés par la colonne de droite, manifestent une véritable alternance codique entre les deux situations de communication: en situation d'examen, ils réalisent un ne sur deux (bleu $21 \%+n e$; vert 21\%-ne), tandis qu'ils manifestent, dans l'immédiat communicatif, une réalisation de $n e$ aussi basse que celle des Suisses.

Nous avons donc établi un résultat intermédiaire important: en situation d'examen, les Français contrôlent beaucoup plus leur production de ne que les Suisses. La différence diatopique (non significative) entre les Suisses et les Français quant à la réalisation de ne détectée en section 4.3.1 reflète donc uniquement le comportement distinct que les Suisses et les Français manifestent dans les situations de distance communicative (ce dernier diffère de manière significative $p=0,002$ ). Ceci signifie que, dans l'immédiat communicatif, il n'y a aucune différence entre la production de ne d'un Suisse et d'un Français. Les différences ne se manifestent que dans la distance communicative: tandis que les Français insèrent, consciemment ou non, un nombre élevé de ne pendant un examen oral, les Suisses ne le font pas.

Nous tenons à aller encore un peu plus loin dans l'exploitation du facteur de la situation de communication dans notre corpus et pour 
cela, nous allons nous servir des profils individuels des 15 locuteurs qui ont été enregistrés aussi bien dans les situations de distance que de l'immédiat communicatif.

À l'aide du diagramme (128), dans lequel chaque colonne représente la production absolue de négations par locuteur, nous pouvons comparer leur production de ne pendant les examens et les conversations : les réalisations de ne en situation d'examen figurent en bleu et celles en situation de conversation en rouge; les omissions de ne pendant un examen sont représentées en vert et en violet pendant une conversation.

(128) Les profils variationnels individuels de 15 locuteurs

La réalisation individuelle de ne par situation de communication

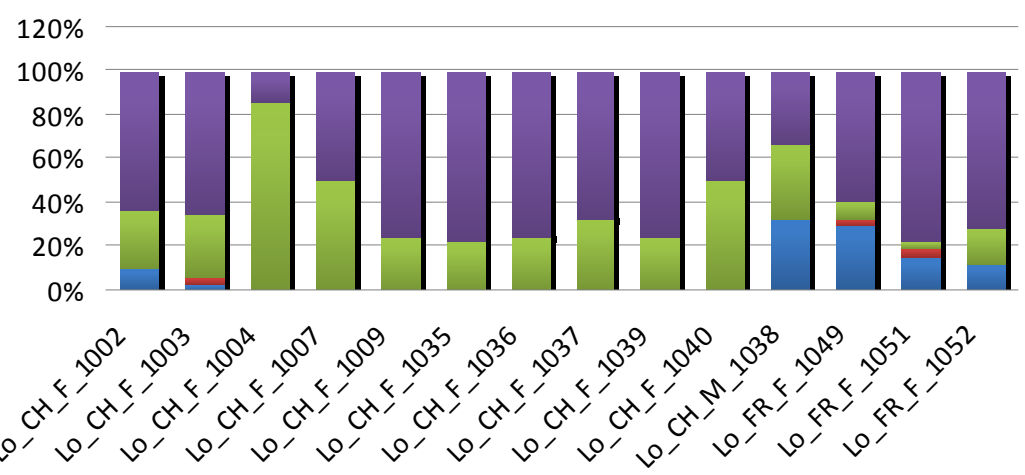

q réalisé examen $\square$ réalisé conversation $\square$ omis examen $\square$ omis conversation

Plus de la moitié des personnes en question appartient au type B des locuteurs ( $c f$. section 4.2.2), c'est-à-dire qu'elles ne réalisent jamais la particule de négation, indépendamment de la situation de communication. Leurs colonnes ne se composent que de deux couleurs: les parties violettes, qui indiquent les omissions pendant les conversations, et les parties vertes, qui représententles omissions pendant les examens. Il s'agit notamment des huit étudiantes suisses susceptibles d'avoir généralisé l'absence de ne et qui ont déjà été décrites en section 4.2.2 (de Lo_CH_F_1004 jusqu'à Lo_CH_F_1040, de gauche à droite dans le diagramme). 
Les personnes restantes appartiennent au type A, c'est-à-dire qu'elles manifestent une variation $\pm n e$ (ceci se voit dans le fait que leurs colonnes sont composées de trois ou même de quatre couleurs).

Considérons d'abord les trois colonnes tripartites qui représentent la réalisation de $\pm n e$ d'une assistante-doctorante de l'université Neuchâtel (CH_F_1002), d'un étudiant de la même université (Lo_CH_M_1038) et d'une élève de la banlieue parisienne (Lo_FR_F_1052). Ces trois locuteurs omettent constamment le ne dans les conversations et n'en réalisent qu'environ un sur deux pendant les examens. Ce comportement indique qu'il y a uniquement dans la distance communicative un facteur qui incite à la réalisation de ne ( $c f$. la discussion en section 5.3).

Les trois colonnes restantes se composent de quatre couleurs, ce qui signifie que les locuteurs correspondants exploitent complètement le spectre de variation possible. Il s'agit ici d'une autre assistante-doctorante suisse (Lo_CH_F_1003) et de deux lycéennes françaises (Lo_ FR_F_1049 et Lo_FR_F_1051). Comme la majorité des francophones, ces trois locutrices omettent plus de ne qu'elles n'en réalisent, et ceci se manifeste dans le diagramme par le fait que l'ensemble des parties vertes et violettes est plus grand que celui des parties rouges et bleues. Les parties rouges, c'est-à-dire le résidu inattendu de négations bipartites lors d'une situation de l'immédiat, sont minimes. En effet, nous n'observons qu'une occurrence de + ne par locutrice.

Nous pouvons donc nous demander si ces occurrences inattendues de +ne dans l'immédiat communicatif sont déclenchées par des facteurs linguistiques qui incitent à l'emploi de la particule, comme par exemple par un sujet lourd ( $c f$. sections 2.2.5.1 et 4.4.1.1) ou par une construction subordonnée ( $c f$. sections 2.2.5.6 et 4.4.5.1).

(129) Le résidu de +ne en situation d'immédiat

\begin{tabular}{|llllll|}
\hline ID & Exemple & Situation & Sujet & Temps & Phrase \\
\hline 1248 & $\begin{array}{l}\text { ma question n'a rien à voir avec ce que t'es } \\
\text { en train de dire }\end{array}$ & imméd & lex & ind. prés. & prin \\
1244 & je ne veux pas & imméd & cl.lé & ind. prés. & prin \\
2418 & je ne sais pas & imméd & cl.lé & ind. prés. & prin \\
\hline
\end{tabular}


Le tableau sous (129) montre que ceci semble être le cas pour le premier exemple (1248), qui contient un sujet lexical (DP ma question), mais pas pour les deux autres cas, dans lesquels le clitique léger je est employé comme sujet. La discussion en section 5.1.4 montrera que le ne dans l'énoncé 1244 est déclenché pragmatiquement, mais en ce qui concerne l'exemple 2418, aucune motivation morphosyntaxique ou pragmatique pour ne n'est reconnaissable. Notons tout de même, pour le moment, que la réalisation phonétique de cet énoncé est [3ən.se.pa]. Le ne est donc résyllabifié dans la coda de la syllabe précédente, ce qui, comme nous allons voir en chapitre 5.2, facilite sa réalisation.

Finalement, nous observons très clairement dans le diagramme (128) les différences quant à l'emploi de \pm ne dans la distance communicative que manifestent les Français et les Suisses: tandis que la doctorante suisse (Lo_CH_F_1003) y produit seulement une partie minime de négations bipartites, les deux élèves françaises (Lo_FR_F_1049 et Lo_FR_F_1051) les produisent de manière assez conséquente. Dans la production langagière de la doctorante suisse (Lo_CH_F_1003), la réalisation de ne reste marginale dans les deux situations de communication, alors que les deux élèves françaises utilisent systématiquement la réalisation de ne comme une variable d'alternance stylistique entre deux situations. Nous pouvons même soupçonner une intention, ou le besoin inconscient d'impressionner les professeurs de lycée, qui produisent, eux-mêmes, des taux très élevés de $69 \%+n e$.

Toutefois, même les deux Françaises qui manifestent une alternance visible entre les situations de communication n'arrivent pas à une production consistante de ne pendant les examens. Le résidu de -ne en situation d'examen est donc éventuellement à expliquer à l'aide des facteurs linguistiques qui incitent à l'omission de la particule ( $c f$. sections 2.2.5 et 4.4).

(130) Le résidu de -ne de Lo_FR_F_1049 et_1051 en distance communicative

\begin{tabular}{|c|c|c|c|c|c|}
\hline ID & Exemple & Situation & Sujet & Temps & Phrase \\
\hline 0066 & mais c'est que dans un sens & dist & cl.lé & ind. prés. & prin \\
\hline 0068 & car il répond qu'à l'écrit & dist & cl.lé & ind. prés. & sub \\
\hline 0898 & $\begin{array}{l}\text { elle se considère pas comme quelqu'un utile } \\
\text { à la société }\end{array}$ & dist & cl.lou & ind. prés. & prin \\
\hline
\end{tabular}


En effet, deux énoncés négatifs sans ne produits par les deux élèves françaises en situation d'examen sur trois comprennent des sujets clitiques légers: $c e$ et $i l$ ( $c f$. sections 2.2.5.1 et 4.4.1.2).

Dans le premier exemple (0066), le clitique léger ce est par ailleurs employé en combinaison avec un verbe de fréquence élevée (c'est). Dans les séquences préformées de ce type, l'absence de ne est presque inévitable, indépendamment de la situation de communication. Le $i l$ dans l'exemple 0068 est également un clitique léger atone qui peut favoriser l'absence de ne pour des raisons rythmiques ( $c f$. section 5.2). Seul le clitique lourd elle dans le dernier exemple (0898) ne constitue pas en soi un obstacle à la réalisation de ne. Néanmoins, dans le cas cité en (130), elle est suivi par le clitique réfléchi se, ce qui alterne la prosodie de la séquence et provoque l'omission de ne (Sturm 1981: 134-135 est le premier à remarquer la tendance à l'omission de ne dans les séquences sujet-lourd +clitique-léger \pm ne, qui, comme nous montrerons dans les sections 5.2.4.3 et 5.2.4.4, est motivée prosodiquement).

Avant de conclure cette section dédiée à l'influence de la situation de communication sur $\pm n e$, nous tenons encore à comparer deux sous-corpus de l'immédiat communicatif enregistrés dans l'un des deux lycées de la région parisienne ( $c f$. section 3.3.2). Il s'agit, d'une part, de conversations libres, menées par les élèves seuls pendant les pauses, dans les couloirs ou dans la salle d'attente pour les examens; et de l'autre, de discussions relativement détendues en classe, enregistrées pendant les cours de l'assistante de langue, qui est la chercheuse.

Afin de déterminer s'il y a une différence entre ces deux types de discours de l'immédiat, nous avons comparé les taux de ne dans les deux sous-corpus. Dans le diagramme (131)a, la colonne de gauche contient les valeurs des personnes enregistrées en conversation libre et la colonne de droite, celles des discussions en classe. 
(131) La réalisation de ne selon la situation de communication en $\mathrm{CH}$ et $\mathrm{F}$

a. Pourcentages

$\pm n e$ dans les conversations libres et les discussions en classes

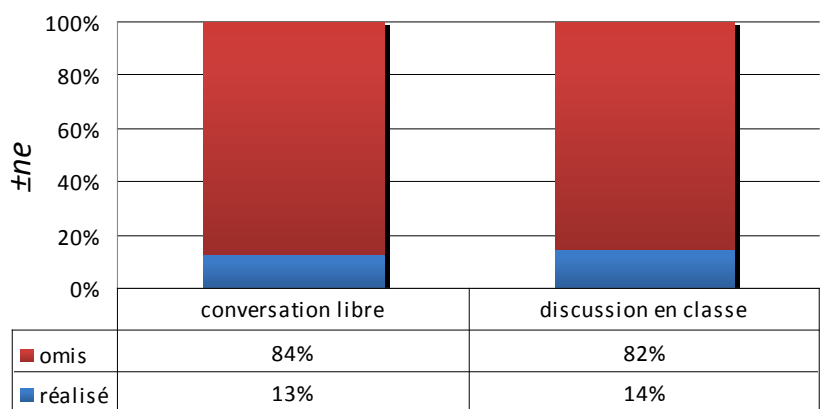

b. Données brutes

\begin{tabular}{|lrrrc|}
\hline & $+n e$ & $-n e$ & Total & $\chi^{2}$ \\
\hline Conversation libre & 4 & 27 & 32 & $p=0,796$ \\
Conversation en classe & 12 & 69 & 84 & \\
Total & 16 & 96 & 116 & \\
\hline
\end{tabular}

Dans la diagramme (131)a, nous observons une ressemblance frappante entre les deux colonnes: dans les conversations libres, 13\% des négations sont monopartites et $14 \%$ le sont lors des conversations en classe. La présence de l'assistante de langue ne provoque donc, contrairement à celle d'un professeur pendant un examen, aucun effet mesurable sur la production de ne des élèves, ce qui est confirmé par le test chi-carré qui n'indique aucune différence significative entre les deux sous-corpus. Ce résultat confirme notre supposition, formulée dans la section 2.4.4.4, selon laquelle la seule présence d'un chercheur n'est pas suffisante pour alterner le comportement des locuteurs. C'est donc probablement pour cette raison qu'Armstrong 2002 n'a trouvé aucune différence dans la production $\pm n e$ des élèves qu'il a testé de cette manière.

Nous pouvons conclure que le regroupement des deux sous-corpus sous la catégorie de l'immédiat ou des conversations effectué en T-zéro est absolument justifié dans la perspective adoptée ici: quant à la réali- 
sation du ne de négation, les élèves français de notre corpus ne semblent guère faire de distinction entre les deux types de conversation.

En somme, nous pouvons retenir qu'il existe une différence significative entre l'immédiat et la distance communicative. Ce sont en particulier les locuteurs français qui effectuent de véritables alternances codiques ou plutôt stylistiques entre les conversations et les examens, tandis que ce comportement est inconnu à la majorité des locuteurs suisses. En situation d'examen, certains élèves français omettent $n e$ très rarement et seulement en combinaison avec des facteurs linguistiques qui le défavorisent. Par contre, à l'intérieur du domaine de l'immédiat, la présence d'une assistante de langue dans la salle ne provoque aucune modification de la réalisation de $n e$.

\subsubsection{Conclusion intermédiaire: l'influence des facteurs extralinguistiques}

En ce qui concerne l'influence des facteurs extralinguistiques sur $\pm n e$, nous pouvons retenir que trois facteurs ont une influence statistiquement significative, donc non-aléatoire, dans notre corpus.

Il s'agit, premièrement, de la situation de communication: la production de ne est plus élevée dans la distance communicative que dans l'immédiat, mais toujours loin d'être conséquente. Deuxièmement, la combinaison entre la provenance géographique et la situation de communication produit une différence significative: il est certes vrai que les Suisses produisent la particule plus rarement que les Français (comme le soutient par exemple Fonseca-Greber 2007), mais cette différence ne se manifeste de manière significative que dans la distance communicative. Dans l'immédiat, le comportement des locuteurs suisses et français est très similaire. Finalement, le statut bi- ou monolingue des locuteurs semble décisif: nous avons détecté ici une tendance intéressante, éventuellement due à un effet de contact langagier, qui n'a pas été thématisée auparavant, à savoir l'absence de ne dans la production des locuteurs bilingues, qui sera approfondie dans la section 5.1.5. 
En revanche, la provenance géographique seule ainsi que l'âge et l'éducation des locuteurs ne produisent pas de différences statistiquement significatives. Bien sûr, ceci ne veut pas dire que ces facteurs ne jouent aucun rôle: l'absence de signification statistique pourrait être due, tout simplement, à la composition démographique de notre corpus (cf. section 3.2).

\subsection{Analyse descriptive de l'influence des facteurs linguistiques}

\subsubsection{L'influence du type de sujet}

Nous avons établi en section 2.2.5.1 qu'une distinction binaire pronominal vs. lexical, telle qu'elle est usuelle pour le type de sujet précédant un prédicat verbal nié dans les analyses de corpus portant sur $\pm n e$ ( $c f$. Ashby 1976, 1981, 2001, Armstrong/Smith 2002, Coveney ${ }^{2} 2002$, Hansen/ Malderez 2004 etc.), n'est pas suffisante. Les sujets forment plutôt un continuum entre deux pôles et sont donc mieux décrits comme sujets légers et lourds.

Ceci se manifeste, tout d'abord, dans le fait que les taux de ne augmentent proportionnellement et régulièrement avec le poids prosodique du sujet. Ensuite, nous avons montré en section 2.2.5.2 qu'également aux niveaux syntaxique, morphologique et phonologique les sujets lexicaux et pronominaux ne forment pas une dichotomie mais plutôt un spectre continu. Par conséquent, il faut effectuer une distinction plutôt prosodique entre les sujets simples qui peuvent être légers (par exemple $j e, t u, c e, i l$ ) ou lourds (par exemple nous, qui, le chien, quelqu'un) et les distinguer des sujets redoublés ( $c f$. section 2.2.5.2) ${ }^{118}$.

118 La simple comparaison des taux de ne des sujets pronominaux et lexicaux provoque elle aussi une différence statistiquement très significative $(p>0.000)$, ce qui indique l'importance du type de sujet pour la réalisation de ne. Dans notre 
La comparaison globale de ces trois types de sujet comme facteur déclenchant une (non-)réalisation du ne en (132) révèle des différences très significatives $(p<0.000)$. Comme l'on s'y attendrait en connaissant les résultats d'autres analyses, la réalisation de ne avec les sujets légers est extrêmement basse. En revanche, le taux de $n e$ avec les sujets lourds dépasse de peu la moitié des énoncés. Finalement, et ceci est peut-être le résultat le plus frappant, avec les sujets redoublés, nous ne trouvons aucune négation bipartite dans notre corpus.

(132) \pm ne selon le type de sujet

\begin{tabular}{|lcrrrrrr|}
\hline & Sujet & \multicolumn{1}{c}{$+n e$} & \multicolumn{2}{c|}{$-n e$} & Total & $\chi^{2}$ \\
\hline \multirow{2}{*}{ Simple } & léger & 14 & $6 \%$ & 206 & $94 \%$ & 220 & $p<0,000$ \\
& lourd & 37 & $45 \%$ & 45 & $55 \%$ & 82 & \\
Redoublé & & 0 & $0 \%$ & 29 & $100 \%$ & 29 & \\
& & 51 & $15 \%$ & 280 & $85 \%$ & 331 & \\
\hline
\end{tabular}

L'élévation régulière et constante des taux de $+n e$ dans le diagramme (133) montre qu'il y a effectivement un continuum entre les sujets légers et lourds et que, comme l'on s'y attendrait après les discussions dans les sections 2.2.5.1 et 2.2.5.2, ces deux catégories forment plutôt les extrémités d'une échelle que deux groupes disjoints ${ }^{119}$.

corpus, les sujets pronominaux comportent une moyenne de $15 \%+n e$ et les sujets lexicaux, une moyenne de $73 \%+n e$. Le faible taux $+n e$ avec les sujets pronominaux s'explique presque exclusivement par le grand nombre de sujets clitiques parmi eux. Les clitiques, surtout ceux que nous classons comme clitiques légers, sont depuis les premières analyses de $\pm n e$ une entrave connue à la réalisation de ne ( $c f$. résumé des études en section 2.2.5.1).

119 Comme nous l'avons déjà mentionné, ce continuum n'est pas du tout une idiosyncrasie de notre corpus et s'observe également dans des corpus beaucoup plus grands ( $c f$.par exemple les résultats de Meisner 2010: 1949, basés sur 2432 occurrences négatives du corpus C-ORAL-ROM de 440.000 mots dans l'annexe IX). 
(133) Le continuum $\pm n e$ entre les sujets légers et lourds

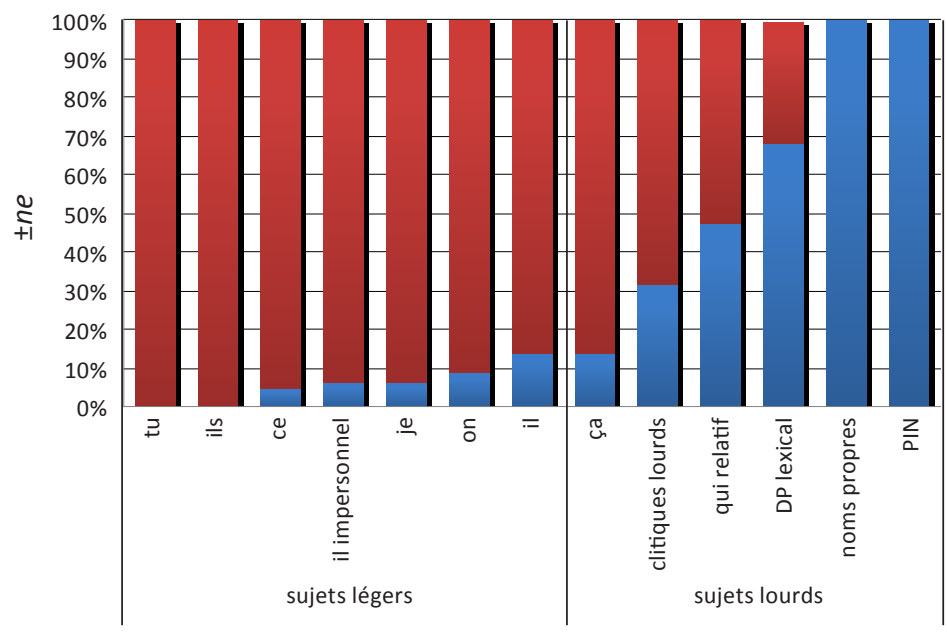

Après cette vue d'ensemble de l'influence des types de sujet, nous considérons dans les sections suivantes les taux de ne en fonction des sujets lourds, légers et redoublés en détail.

\subsubsection{Les sujets lourds}

Considérons, tout d'abord, les sujets lourds des énoncés négatifs dans notre corpus en (134).

(134) $\pm n e$ avec les sujets lourds

a. Pourcentages

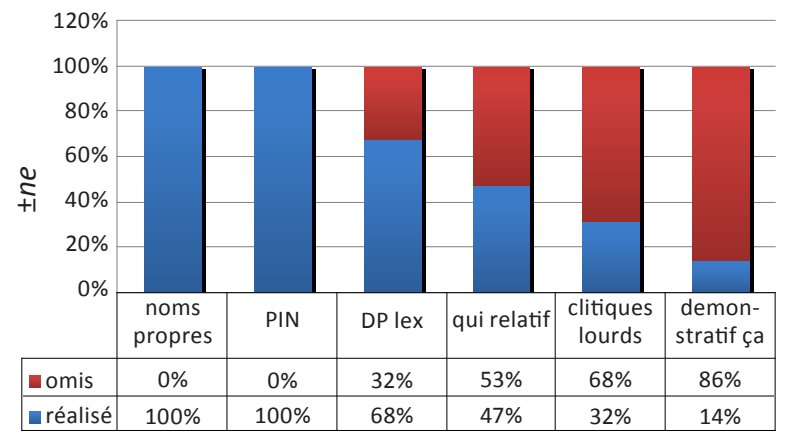


b. Données brutes

\begin{tabular}{|lrrrrrc|}
\hline Sujets lourds & $+n e$ & & $-n e$ & \multicolumn{2}{c|}{ total } & $\chi^{2}$ \\
\hline noms propres & 3 & $100 \%$ & 0 & $0 \%$ & 3 & $p=0,001^{120}$ \\
PIN & 2 & $100 \%$ & 0 & $0 \%$ & 2 & \\
DP lex & 13 & $68 \%$ & 6 & $32 \%$ & 19 & \\
qui relatif & 9 & $47 \%$ & 10 & $53 \%$ & 19 & \\
clitiques lourds & 8 & $32 \%$ & 17 & $68 \%$ & 25 & \\
démonstratif ça & 2 & $14 \%$ & 12 & $86 \%$ & 14 & \\
total & 37 & $45 \%$ & 45 & $55 \%$ & 82 & \\
\hline
\end{tabular}

En (134), nous observons un net déclin des taux de ne à partir des noms propres et des pronoms indéfinis négatifs, qui sont, dans notre corpus, toujours réalisés avec la particule ne, comme les exemples (135)a et b le montrent. Ces deux types de sujet sont suivis par les DP lexicaux, illustrés en (135)c, qui manifestent un taux de $68 \%+n e$. Ces trois types de sujet favorisent donc très clairement la présence de $n e$.

(135) Les sujets lourds qui favorisent la réalisation de ne dans plus de $50 \%$ des cas

a. Les noms propres: car Ulysse n'écoute pas le récit de Cornélius (0034)

b. Les PIN : personne ne s'est mû (1472)

c. Les DP lexicaux: la mère ne sait pas qu'elle s'est séparée (0796)

Par contre, le pronom relatif qui (cf. (136)a, 47\% +ne), les clitiques lourds elle (cf. 35\% +ne) et vous ( $c f .(136)$ c, 33\% +ne) provoquent moins de négations bipartites que monopartites. Toutefois, ces pronoms dépassent toujours largement, avec une moyenne de $32 \%+n e$, la moyenne du corpus entier de $18 \%+n e$.

(136) Les sujets lourds qui favorisent la réalisation de ne dans plus de $30 \%$ des cas

a. Le pronom relatif qui: une narratrice qui ne sert à rien (0978)

b. Le clitique lourd elle: elle n'est pas religieuse (1537)

c. Le clitique lourd vous: si vous ne voulez pas après vous pouvez dire (1473)

120 Les groupes des noms propres, des PIN et des DP lexicaux ont été regroupés pour l'application du test chi-carré afin de créer des chiffres suffisamment élevés. 
Le démonstratif ça est avec 14\% +ne le seul sujet lourd qui montre un taux de ne inférieur à la moyenne du corpus, c'est-à-dire que les exemples sans ne comme celui en (137)a sont beaucoup plus nombreux dans le corpus que ceux contenant la particule comme en (137)b. On pourrait donc conclure que, quant à la variable $\pm n e, c ̧ a$ se comporte plutôt comme un sujet léger.

(137) Le sujet lourd ça qui incite comme les sujets légers à l'omission de ne

a. $\quad$ ça va pas de soi (0409)

b. ça ne me dérange pas (1903)

De même, au niveau morphologique, le pronom monosyllabique ça, composé d'une attaque consonantique et d'un noyau vocalique, ressemble fortement aux clitiques légers je, tu et $c e$, mais contrairement à ceux-ci, au niveau syntactico-phonologique, ça reste syntaxiquement séparable du verbe fléchi, s'emploie dans l'isolement et est accentuable (cf. section 2.2.5.2).

En tenant compte de ce que nous avons appris jusqu'à présent, l'on pourrait présumer l'existence d'une tendance globale selon laquelle la longueur du sujet influence clairement la réalisation de ne. Afin de contrôler si une telle tendance existe, nous présentons dans le diagramme (138) les taux de réalisation de ne par nombre de syllabes des sujets lourds (pour plus de détails, $c f$. la liste des sujets lourds en annexe X).

(138) $\pm n e$ en fonction du nombre de syllabes des sujets lourds

a. Pourcentages

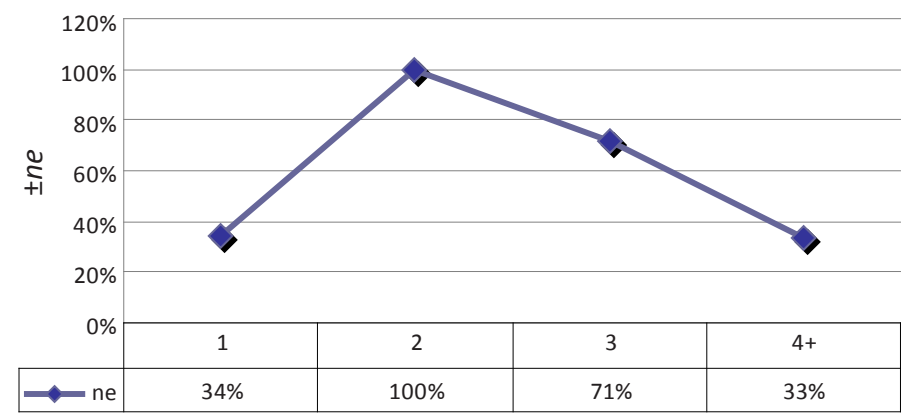


b. Données brutes

\begin{tabular}{|lccccc|}
\hline Nombre de syllabes & 1 & 2 & 3 & $4+$ & $\chi^{2}$ \\
\hline Réalisé & 20 & 9 & 5 & 3 & $p=0,001^{121}$ \\
& $34 \%$ & $100 \%$ & $71 \%$ & $33 \%$ & \\
Omis & 39 & 0 & 2 & 6 & \\
& $66 \%$ & $0 \%$ & $29 \%$ & $67 \%$ & \\
Total & 59 & 9 & 7 & 9 & \\
\hline
\end{tabular}

Nous observons effectivement des différences significatives entre les différentes longueurs de sujets. Il y a une hausse de ne surtout avec les sujets dissyllabiques, mais également avec ceux qui sont trisyllabiques, tandis qu'aussi bien les sujets monosyllabiques que les sujets polysyllabiques (composés de plus de 4 syllabes) conduisent à une réalisation plus restreinte de $n e$.

Par conséquent, pour les sujets mono- et dissyllabiques, il y a un parallélisme net entre la longueur du sujet et la probabilité que ne soit réalisé. Avec un sujet monosyllabique, bien que phonologiquement lourd, l'omission de ne est probable, alors que les DPs dis- ou trisyllabiques s'associent généralement à la particule $n e$.

Toutefois, cette tendance semble ne plus être opératoire lorsqu'un sujet a une longueur supérieure à deux syllabes, c'est-à-dire que la relation biunivoque entre la longueur d'un sujet et la probabilité que ne soit présent n'est pas indéterminée. Il faut noter que pour les DPs plus complexes il est difficile d'établir des tendances sur la base d'un corpus spontané, car celles-ci sont plutôt rares dans ce type de données ${ }^{122}$. $\mathrm{Au}$ vu de la nature éventuellement fortuite de ces observations, nous nous limitons donc à mentionner qu'il existe une relation stable entre la longueur d'un sujet jusqu'à deux syllabes et la réalisation de ne: plus le sujet est long, plus la réalisation de ne est probable.

121 Les groupes des sujets tri- et quadrisyllabiques ont été regroupés pour l'application du test chi-carré afin de créer des chiffres suffisamment élevés.

122 Dans les DPs plus longs, leur structure interne peut éventuellement provoquer des différences quant à la réalisation de ne. Il vaudrait donc la peine de vérifier nos observations par le biaisd'une analyse pointue sur ce facteur (par exemple à travers un sondage). 
Dans ce sous-chapitre, nous avons vu que les sujets lourds forment, eux aussi, un continuum quant à la variable $\pm n e$. Ils provoquent (jusqu'à une longueur de deux syllabes) la réalisation de la particule de manière proportionnelle à leur propre longueur, c'est-à-dire qu'également à l'intérieur du groupe des sujets lourds, les sujets dissyllabiques sont beaucoup plus susceptibles de déclencher l'emploi de ne que les monosyllabiques. De fait, les sujets bisyllabiques atteignent des taux de $100 \%$ +ne dans notre corpus. Nous laissons aux futurs travaux de recherche la tâche d'établir l'influence des sujets polysyllabiques dans les énoncés négatifs, car un sondage, voire des tests d'intuition ( $c f$. Coveney 1998, Meisner 2013) se prêteraient mieux à cet objectif qu'une analyse de corpus.

\subsubsection{Les sujets légers}

La majorité des sujets dans les énoncés négatifs sont des sujets dits légers (66\%), qui devraient provoquer, indépendamment de la situation de communication, des taux très restreints, voire une réalisation zéro de ne ( $c f$. section 2.2.5.1). Les chiffres sous (139) montrent que cette attente est complètement comblée dans notre corpus.

(139) \pm ne avec les sujets légers

a. Pourcentages

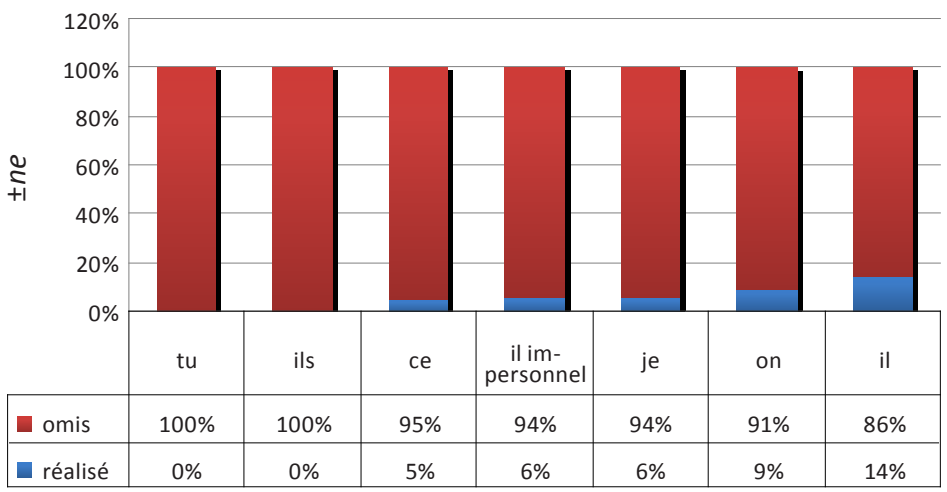


b. Données brutes

\begin{tabular}{|crrrrrr|}
\hline Clitique & \multicolumn{2}{c}{+ ne } & \multicolumn{2}{c|}{$-n e$} & Total & \\
\hline$t u$ & 0 & $0 \%$ & 12 & $100 \%$ & 12 & $p=0,565$ \\
$i l s$ & 0 & $0 \%$ & 7 & $100 \%$ & 7 & \\
ce & 2 & $5 \%$ & 39 & $95 \%$ & 41 & \\
il impersonnel & 1 & $6 \%$ & 15 & $94 \%$ & 16 & \\
je & 5 & $6 \%$ & 89 & $94 \%$ & 94 & \\
on & 2 & $9 \%$ & 20 & $91 \%$ & 22 & \\
$i l$ & 4 & $14 \%$ & 24 & $86 \%$ & 28 & \\
Total & 14 & $6 \%$ & 206 & $94 \%$ & 220 & \\
\hline
\end{tabular}

Les clitiques $t u$ et $i l s$, illustrés par les exemples en (140)a et b, n'apparaissent jamais en combinaison avec le clitique de négation.

(140) Les clitiques légers qui provoquent une réalisation minime, voire zéro de $n e$

a. $\quad$ tu peux pas t'asseoir (0758)

b. $\quad$ ça les critiques ils ont pas du tout accepté qu'on montre quelque chose comme ça (2178)

c. c'est pas vraiment habile de faire un plan (0444)

d. est-ce qu'avant il y avait pas déjà deux codes un oral et un écrit (0059)

e. j'ai pas grand-chose à dire

f. on peut pas faire les bruits des animaux (0656)

g. $\quad$ parce qu'il a rien d'autre après (0208)

Les pronoms ce, il impersonnel et je (cf. (140)c-e) manifestent eux aussi avec respectivement $5 \%, 6 \%$ et $6 \%+n e$ une forte tendance générale à l'omission de ne. Les taux de on et il $(9 \%$ et $14 \%+n e)$ sont légèrement plus élevés, mais toujours inférieurs à la moyenne du corpus, qui s'élève à $18 \%$. Il est important de noter que ces observations sont valables pour l'ensemble du corpus: même dans les situations de distance communicative, il y a une réalisation très basse ou zéro de ne avec ces éléments. De plus, le tableau en (139)b montre un autre fait intéressant: il n'y a aucune différence significative entre les clitiques légers en ce qui concerne $\pm n e$. Ceci signifie que, contrairement aux sujets lourds, les sujets légers semblent former un bloc relativement homogène, du moins en ce qui concerne la variable $\pm n e$. 
(141) \pm ne selon la variante du sujet léger

a. Pourcentages

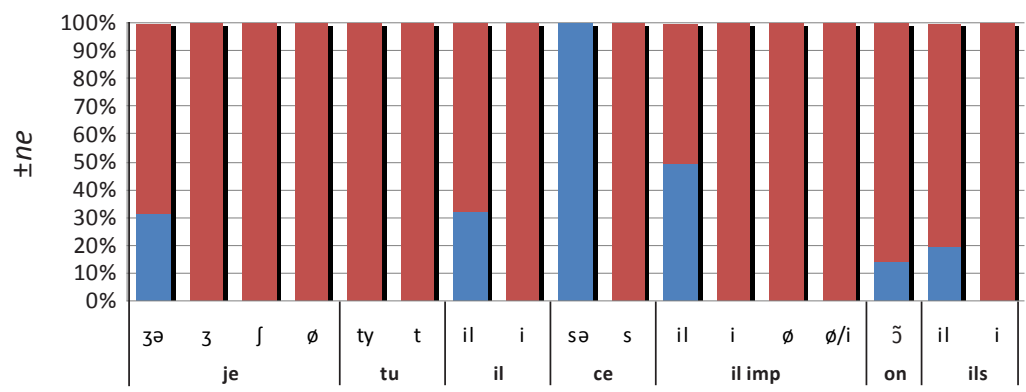

b. Données brutes

\begin{tabular}{|c|c|c|c|c|c|c|c|c|}
\hline & & $+n e$ & & $-n e$ & & Total & $\chi^{2}$ & Total par pronom \\
\hline \multirow[t]{4}{*}{ je } & 32 & 5 & $32 \%$ & 13 & $68 \%$ & 19 & \multirow[t]{4}{*}{$p<0,000^{123}$} & 94 \\
\hline & 3 & 0 & $0 \%$ & 52 & $100 \%$ & 52 & & \\
\hline & $\int$ & 0 & $0 \%$ & 23 & $100 \%$ & 23 & & \\
\hline & $\varnothing$ & 0 & $0 \%$ & 1 & $100 \%$ & 1 & & \\
\hline \multirow[t]{2}{*}{$t u$} & ty & 0 & $0 \%$ & 9 & $100 \%$ & 9 & & 12 \\
\hline & $\mathrm{t}$ & 0 & $0 \%$ & 3 & $100 \%$ & 3 & & \\
\hline \multirow[t]{2}{*}{ il } & il & 8 & $32 \%$ & 17 & $68 \%$ & 25 & & 28 \\
\hline & $\mathrm{i}$ & 0 & $0 \%$ & 3 & $100 \%$ & 3 & & \\
\hline \multirow[t]{2}{*}{ ce } & sə & 2 & $100 \%$ & 0 & $0 \%$ & 2 & & 41 \\
\hline & $\mathrm{s}$ & 0 & $0 \%$ & 39 & $100 \%$ & 39 & & \\
\hline \multirow[t]{4}{*}{ il imp } & il & 2 & $50 \%$ & 2 & $50 \%$ & 4 & & 16 \\
\hline & $\mathrm{i}$ & 0 & $0 \%$ & 2 & $100 \%$ & 2 & & \\
\hline & $\varnothing$ & 0 & $0 \%$ & 9 & $100 \%$ & 9 & & \\
\hline & $\varnothing / i$ & 0 & $0 \%$ & 1 & $100 \%$ & 1 & & \\
\hline on & $\tilde{\jmath}$ & 3 & $14 \%$ & 19 & $86 \%$ & 22 & & 22 \\
\hline \multirow[t]{2}{*}{$i l s$} & il & 1 & $20 \%$ & 4 & $80 \%$ & 5 & & 7 \\
\hline & $\mathrm{i}$ & 0 & $0 \%$ & 2 & $100 \%$ & 2 & & \\
\hline Total & & 21 & $10 \%$ & 199 & $90 \%$ & 220 & & 220 \\
\hline
\end{tabular}

123 Variantes standard vs. non-standard (les cas peu clairs comme [3] ont été exclus). 
Contrairement aux analyses précédentes de \pm ne ( $c f$. section 2.2.2), notre corpus permet d'accéder à l'influence respective des divers allomorphes clitiques, comme les données en (141) le montrent.

Nous observons une différence extrêmement significative entre les variantes standard et non-standard des clitiques. Pour de nombreux clitiques, les taux de ne diffèrent clairement entre la forme standard et les variantes allomorphiques. Dans certains cas, la particule ne apparaît uniquement avec la réalisation standard des pronoms personnels, à savoir avec les formes suivantes: [3ə] pour je, [il] pour il(s) et [sə] pour ce. À l'inverse, les variantes suivantes ne se combinent jamais avec ne: [3] et [ $\left.\int\right]$ pour je, l'ellipse de je ou de il impersonnel (représentée dans le diagramme par le signe $<\varnothing>$ ) et les variantes [i] pour il(s) et [s] pour $c e$. Le seul pronom qui ne manifeste aucune différence de \pm ne entre ses deux formes de réalisation est $t u$ : le taux de ne est zéro aussi bien avec [ty] qu'avec [t].

Nous pouvons donc résumer l'influence des sujets légers sur la réalisation de ne comme suit: de façon générale, les taux globaux de ne avec un sujet léger sont inférieurs à la moyenne du corpus et s'il y a plusieurs variantes d'un clitique, la négation s'associe alors exclusivement avec la forme standard, tandis que les autres variantes sont toujours réalisées sans $n e$.

\subsubsection{Les sujets redoublés}

Dans notre corpus, 29 des sujets redoublés se trouvent dans les énoncés négatifs analysés, ce qui correspond à $9 \%$ de tous les énoncés négatifs. Comme nous l'avons mentionné dans la section 4.4.1 (cf. tableau (132)), aucun de ces cas ne contient la particule ne, ce qui pourrait peut-être signifier qu'il s'agit d'une incompatibilité principale entre les sujets redoublés et le clitique ne, comme le soutient par exemple Massot (2010).

Le tableau en (142) montre que trois quarts des sujets redoublés contiennent un élément non-clitique, c'est-à-dire un pronom tonique ou un DP, en position préverbale ( $c f$. sa fille elle veut pas, 0624), et que seulement un quart des éléments non-clitiques redoublés apparaissent après le verbe (cf. c'est pas mal les Autrichiens, 0756). 
(142) Les sujets redoublés dans les énoncés négatifs

\begin{tabular}{|lrr|}
\hline XP préverbal & 22 & $76 \%$ \\
XP postverbal & 7 & $24 \%$ \\
\hline Total & 29 & $100 \%$ \\
\hline
\end{tabular}

Ce rapport de 3 à 1 des sujets redoublés pré- et postverbaux reflète une tendance générale du corpus. La distribution des éléments non-clitiques redoublés dans l'ensemble du corpus est presque pareille à celle observée pour les énoncés négatifs:

(143) Les sujets redoublés dans T-zéro

\begin{tabular}{|lrr|}
\hline XP préverbal & 204 & $78 \%$ \\
XP postverbal & 54 & $21 \%$ \\
XP pré et postverbal & 2 & $1 \%$ \\
total & 260 & $100 \%$ \\
\hline
\end{tabular}

Quant à la structure interne des sujets redoublés, les diagrammes en (144) montrent que les XPs typiquement redoublés sont les pronoms toniques et les DP lexicaux, suivis par les noms propres et que seule une partie très restreinte des éléments redoublés sont d'autres XP. Le diagramme (144)b montre clairement que la variante [s] du clitique léger $c e$, qui ne se combine jamais avec ne ( $c f$. section 4.4.1.2), est largement la plus récurrente dans les cas de redoublement et qu'elle est suivie par une autre variante prosodiquement légère, à savoir [3]. Dans l'ensemble, $65 \%$ des sujets redoublés contiennent des variantes clitiques monosegmentales, qui semblent incompatibles avec le clitique ne dans notre corpus, comme nous l'avons montré précédemment ( $c f$. section 4.4.1.2). 
(144) La structure interne des sujets redoublés dans le corpus

a. Les XPs redoublés par un pronom

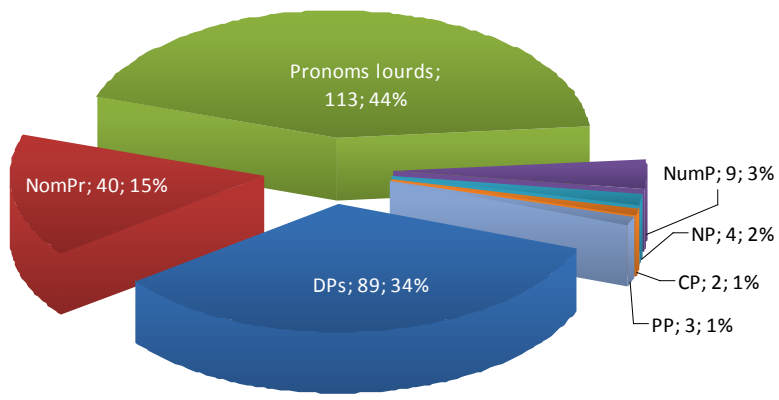

b. Les variantes pronominales redoublées par un XP

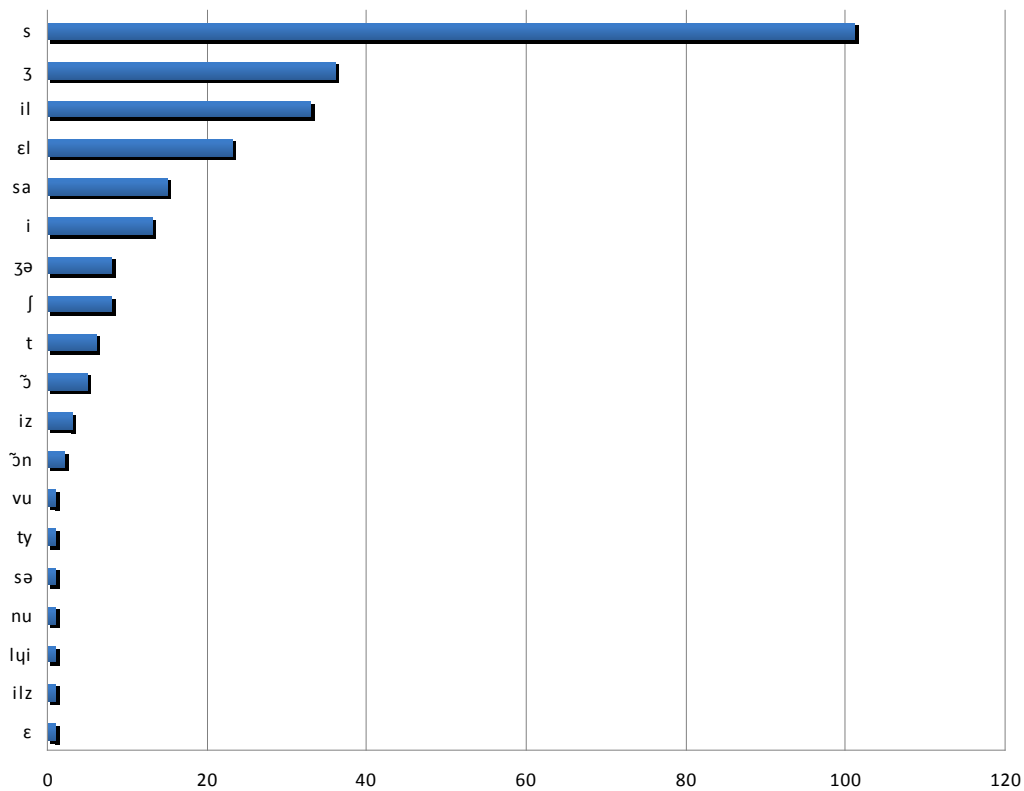

Dans les cas restants, ce sont des variantes bisegmentales légères comme [il], [عl], [sa] ou [3ə], qui font également obstacle à la réalisation de $n e$. 
Avant de conclure cette section, considérons encore les verbes qui s'emploient avec les sujets redoublés:

(145) Les verbes employés avec les sujets redoublés

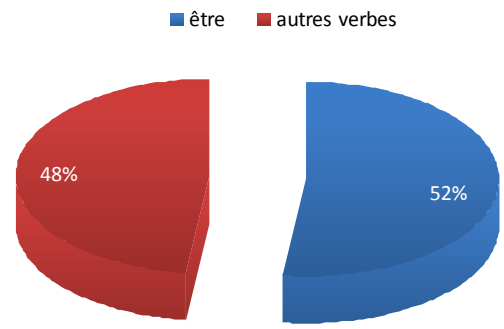

Le diagramme sous (145) montre que dans plus de la moitié des sujets redoublés, le verbe être est employé. Dans $40 \%$ des cas totaux, il se combine avec le clitique démonstratif $c e$ et donne lieu à la séquence préformée c'est, dans laquelle ne est quasiment exclu: dans l'ensemble du corpus, la particule n'est introduite que dans deux des 43 séquences c'est ${ }_{N E G}$ (avec et sans redoublement). Les deux réalisations de ne dans ce contexte ( $c f$. CVs 1517 et 1507 dans T-zéro) sont produites lors d'un examen oral par un professeur universitaire non-natif. Nous pouvons donc conclure que, sauf dans les cas d'hypercorrection, aucun locuteur natif et compétent n'introduirait un ne dans une séquence $\mathrm{XPlourd}+c^{\prime} e s t+\mathrm{NEG}$.

De manière globale, l'absence de $n e$ avec les sujets redoublés n'est donc pas fortuite. Au contraire, nous avons vu qu'elle est due à la forte présence de pronoms sujets prosodiquement légers et en particulier de leurs variantes monosegmentales (qui semblent incompatibles avec ne). Ceci est particulièrement visible dans les structures extrêmement fréquentes XPlourd $+c^{\prime} e s t+\mathrm{NEG}$, dans lesquelles une insertion de $n e$ semble catégoriquement impossible pour les locuteurs natifs sauf dans des cas d'hypercorrection. 


\subsubsection{Les séquences proclitiques}

Bien que la présence des proclitiques soit souvent citée comme un facteur incitant à l'omission de ne, par exemple par les adhérents à l'hypothèse d'une contrainte clitique ( $c f$. section 5.1.2 et Ashby 1977, Culbertson/Legendre 2008, Larrivée 2014, Posner 1985: 189), cette thèse n'est pas clairement confirmée par les corpus existants ( $c f$. section 2.2.5.3).

Dans notre corpus, nous avons trouvé 44 structures niées qui comportent, outre le clitique sujet et la variable $\pm n e$, au moins un autre proclitique. En (146) figurent des exemples pour chaque type de séquence clitique que nous avons trouvé parmi les énoncés négatifs.

(146) $\pm n e$ dans les séquences proclitiques

$\mathrm{S}( \pm n e)+\mathrm{COD}$

a. moi je la regarde même pas (1908)

b. je l'ai pas lu (1246)

$\mathrm{S}( \pm n e)+y$ présentatif

c. et il y avait que cinq minutes qui avaient passé (1940)

d. il n'y a pas beaucoup (0124, examen F)

$\mathrm{S}( \pm n e)+$ réfléchi

e. je me rappelais pas (1100)

f. $\quad$ tu te mets pas là (0766)

$\mathrm{S}( \pm n e)+\mathrm{COI}$

g. $\quad$ mardi elle nous a rien donné (2030)

h. qu'ils lui mettent pas échec (2430)

$\mathrm{S}( \pm n e)+\mathrm{COP}$

i. $\quad$ que la Bretagne elle en fait pas partie (1361)

Dans les séquences proclitiques, la variable \pm ne suit toujours le clitique sujet. La combinaison d'un clitique sujet avec un clitique object direct (le, $\left.l a, l^{\prime}\right)$, illustrée dans les exemples (146)a et b, est la séquence la plus fréquente dans nos données. La séquence proclitique qui la suit immédiatement en termes de fréquence est la combinaison quasi lexicalisée entre le clitique impersonnel $i l$ et le présentatif $y$ illustrée en (146)c et d. Les combinaisons de clitique sujet et des formes du clitique réfléchi me/te/se sont illustrées en e et $\mathrm{f}$. En $\mathrm{g}$ et $\mathrm{h}$ figurent des combinaisons entre des clitiques marquant le sujet et le COI, et en i figure un exemple comprenant le clitique en qui représente un complément prépositionnel. 
Les taux de réalisation de $n e$ dans les séquences proclitiques donnent lieu à une moyenne de $14 \%+n e$, comme le montre le diagramme (147).

(147) $\pm n e$ dans les séquences proclitiques

a. Pourcentages

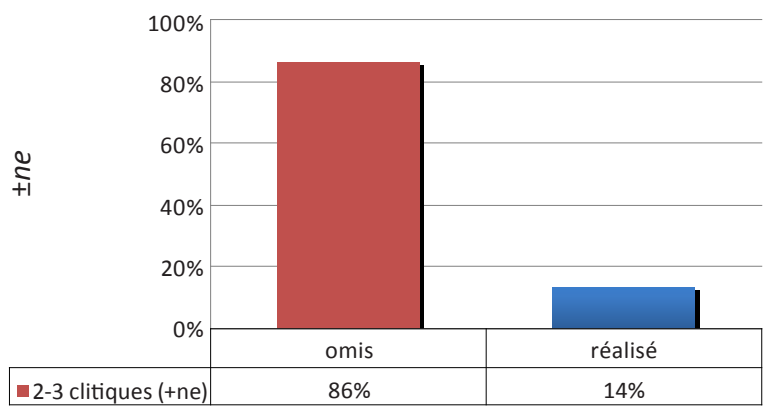

b. Données brutes

\begin{tabular}{|cccccccc|}
\hline & $2 \mathrm{Cl}( \pm n e)$ & & & \multicolumn{5}{c|}{$3 \mathrm{Cl}( \pm n e)$} \\
\hline Sujet + & $\mathrm{COD}$ & $\mathrm{y}$ & réfléchi & $\mathrm{COI}$ & $\mathrm{COP}$ & $\mathrm{COD} /$ réfléchi, COI & total \\
\hline$-n e$ & 18 & 9 & 5 & 4 & 2 & 0 & 38 \\
$\%$ & $95 \%$ & $82 \%$ & $100 \%$ & $80 \%$ & $67 \%$ & $0 \%$ & $86 \%$ \\
\hline$\%$ & $5 \%$ & $18 \%$ & $0 \%$ & $20 \%$ & $33 \%$ & $100 \%$ & $14 \%$ \\
$+n e$ & 1 & 2 & 0 & 1 & 1 & 1 & 6 \\
\hline Total & 19 & 11 & 5 & 5 & 3 & 1 & 44 \\
\hline
\end{tabular}

Dans le diagramme (147)a, la colonne de gauche, en rouge, représente les $86 \%$ des séquences clitiques qui manifestent une négation monopartite, et la colonne bleue représente les $14 \%$ des cas dans lesquels le clitique ne fait partie de la séquence proclitique. Au total, dans les séquences proclitiques, nous détectons une claire tendance à l'omission du ne par rapport à la moyenne du corpus $(18 \%+n e)$. Par contre, par rapport à la réalisation de $n e$ avec un sujet léger, qui est extrêmement basse $(6 \%+n e)$ indépendamment de la présence ou non d'autres clitiques, la présence de ne est élevée dans les séquences proclitiques. Il s'agira donc, par la suite, de comprendre pourquoi il y a un tel déca- 
lage: les séquences proclitiques incitent généralement à l'omission de ne, mais dans une moindre mesure que la simple présence d'un sujet clitique léger.

Selon une première hypothèse, il existe certains proclitiques qui incitent à l'omission de ne et d'autres qui favorisent sa présence. À cet égard, le tableau (147)b montre les différentes combinaisons de clitiques que nous avons trouvées et les taux de ne respectifs. Les séquences en (147)b sont classées selon la fonction grammaticale des clitiques (qui est illustrée par les exemples en (146)) et apparaissent en ordre décroissant, selon leur fréquence. En effet, il semble que les clitiques objets directes et réfléchis favorisent davantage l'omission de ne que le locatif $y$ et ceux représentant le complément d'objet indirect et le complément prépositionnel. Toutefois, comme c'est souvent le cas dans les corpus (cf. section 2.2.5.4), les chiffres totaux pour les diverses séquences proclitiques sont trop bas pour émettre des conclusions robustes.

Selon une deuxième hypothèse, les cas de réalisation de ne dans les séquences proclitiques sont simplement des hypercorrections. Considérons dans ce contexte l'exemple sous (148), qui représente la seule séquence de quatre clitiques dans notre corpus et la plus longue que nous avons trouvée. Comme tous les exemples contenant un ne dans les séquences proclitiques, elle provient d'un examen oral.

(148) La réalisation de ne dans les séquences proclitiques: un hypercorrection? $\begin{array}{ccccccc}\text { tout du moins } & \text { il } & \text { ne } & \boldsymbol{s} & \boldsymbol{y} & \text { intéresse pas } & \text { (0080, examen F) } \\ & \text { S } & \text { NEG } & \text { réfl. } & \text { loc. }\end{array}$

Étant donné que dans les séquences proclitiques la réalisation de ne est généralement improbable par rapport à l'ensemble du corpus et que les occurrences de ne dans ces structures proviennent sans exception des situations d'examen, il semble prudent de soutenir que l'exemple en (148) et toutes les autres ocurrences de ne dans les séquences proclitiques sont des cas d'hypercorrection. De plus, nous allons montrer dans la section 5.2.4.2 que la réalisation de ne dans une séquence proclitique n'est possible que si les clitiques sont réalisés sous leur variante standard et la structure syllabique de la séquence est maintenue. 


\subsubsection{L'élément négatif non-clitique}

L'élément négatif non-clitique est un facteur qui apparaît, comme le type de sujet, dans toute analyse empirique de la réalisation de ne, même si, contrairement à ce dernier, son influence n'est pas clairement établie (cf. section 2.2.5.4). Toutefois, une certaine tendance incontestable qui s'est cristallisée dans les analyses précédentes émerge également de nos données. Il s'agit de l'impact de pas, l'élément négatif le plus fréquent dans tous les corpus, qui semble favoriser l'omission de $n e$.

Dans le tableau (149), nous observons que la réalisation de ne avec la particule négative pas $(17 \%+n e)$ ou ses variantes modifiées ( pas $d u$ tout, pas beaucoup, pas encore) est plus basse qu'avec l'ensemble des autres termes négatifs postverbaux.

(149) \pm ne selon l'élément négatif non-clitique

a. Tous les éléments négatifs

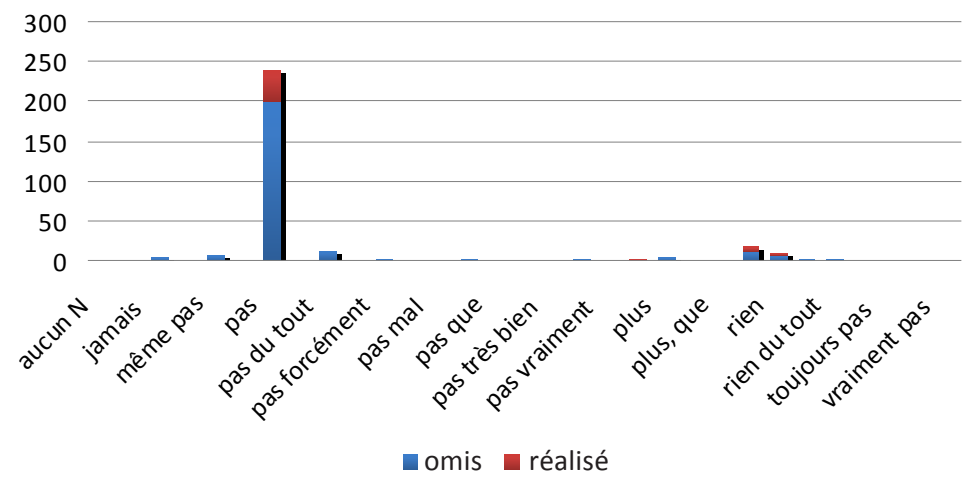

b. La différence entre pas et les autres éléments négatifs

\begin{tabular}{|lccrrrr|}
\hline Élément négatif non-clitique & $-n e$ & $\%$ & $+n e$ & $\%$ & Total & \\
\hline pas & 200 & $83 \%$ & 41 & $17 \%$ & 241 & pas $(+\mathrm{X})$ vs. autres NEG: \\
pas $+\mathrm{x}$ & 24 & $89 \%$ & 3 & $11 \%$ & 27 & $p=0,001$ \\
autres & 51 & $77 \%$ & 15 & $23 \%$ & 66 & \\
Total & 275 & $82 \%$ & 59 & $18 \%$ & 334 & \\
\hline
\end{tabular}


En (149)a, nous observons les occurrences des termes négatifs et les réalisations de $n e$ respectives ( $c f$. annexe XI pour les chiffres exactes) d'une manière plus détaillée. Mis à part la grande présence de pas, nous observons que les autres éléments négatifs sont rares, ce qui rend l'évaluation de leurs influences respectives sur \pm ne particulièrement difficile.

De manière générale, nous constatons que l'omission de ne est possible avec tout élément négatif, mais elle est significativement plus fréquente avec pas qu'avec d'autres éléments.

Il est fort possible que la fonction grammaticale des pronoms négatifs personne et rien, en tant que sujet ou objet, provoque une différence. Effectivement, les deux occurrences de personne dans le corpus se distinguent notamment par leur fonction et par la (non-)réalisation de $n e$.

(150) L'influence de la fonction grammaticale de personne sur \pm ne

a. personne $e_{\mathrm{Su}}$ ne s'est mû (1472)

b. vous avez vu personne $\mathrm{COD}_{\mathrm{C}}$ ici en haut ce matin (0563)

Comme l'on s'y attendrait en connaissant l'influence du type de sujet (cf. section 2.2.5.1), ne est réalisé précisément là où un élément prosodiquement lourd, dans ce cas l'indéfini négatif personne, se trouve en position de sujet.

Dans l'ensemble, comme la réalisation de ne avec l'élément négatif le plus fréquent pas correspond quasiment à la moyenne du corpus, nous supposons que l'élément négatif en soir n'exerce aucune influence puissante sur la variable $\pm n e$. Il semble toutefois possible que la position pré- ou postverbale ainsi que la fréquence de certains éléments négatifs jouent un rôle.

\subsubsection{Le verbe}

L'analyse de l'influence du type de sujet sur $\pm n e$ a montré que le contexte gauche de la variable est très puissant. Afin de tester également le rôle de la fréquence des éléments dans le contexte immédiatement à droite de $\pm n e$, nous comparons ses taux de réalisation selon la fréquence du verbe. 
En (151), les verbes et leurs taux de réalisation de ne respectifs sont classés par ordre croissant, selon le nombre total d'occurrences dans le corpus. Les constructions négatives de T-zéro contiennent 81 verbes différents. Tandis que les verbes à haute fréquence (jusqu'à 100-200 occurrences) manifestent des taux de ne plutôt bas, les verbes les moins fréquents (1-10 occurrences) manifestent des taux de réalisation plus élevés $(68 \%+n e)$. Néanmoins, ces tendances globales ne s'appliquent pas à avoir et être: le taux de réalisation de ne avec avoir (200-300 occurrences) reflète parfaitement la moyenne du corpus $(18 \%+n e)$, tandis que celui avec être ( $>650$ occurrences) reste légèrement inférieur $(15 \%+n e)$.

(151) \pm ne selon la fréquence du verbe

a. Pourcentages

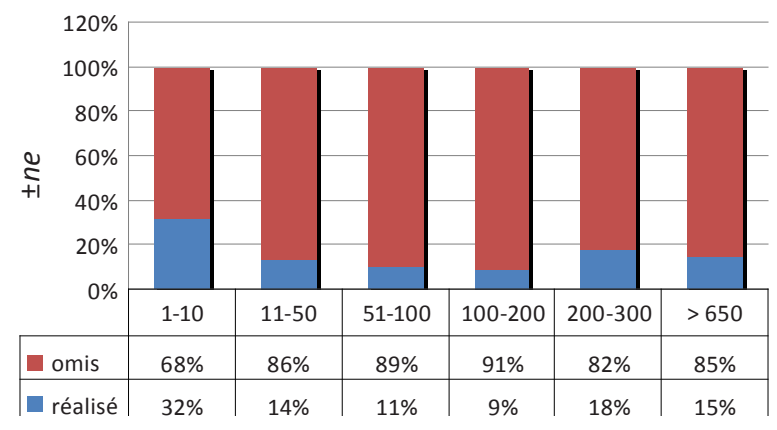

b. Données brutes

\begin{tabular}{|lclrlrrrrr|}
\hline $\begin{array}{l}\text { Occurrences } \\
\text { dans T-zéro }\end{array}$ & No. de verbes & Exemple & $-n e$ & $\%$ & $+n e$ & $\%$ & Total & $\chi^{2}$ \\
\hline $1-10$ & 55 & convaincre & 53 & $68 \%$ & 25 & $32 \%$ & 78 & $p=0,008$ \\
$11-50$ & 18 & devoir & 38 & $86 \%$ & 6 & $14 \%$ & 44 & \\
$51-100$ & 4 & savoir & 82 & $89 \%$ & 10 & $11 \%$ & 92 & \\
$100-200$ & 2 & faire, dire & 10 & $91 \%$ & 1 & $9 \%$ & 11 & \\
$200-300$ & 1 & avoir & 23 & $82 \%$ & 5 & $18 \%$ & 28 & \\
$>650$ & 1 & être & 69 & $85 \%$ & 12 & $15 \%$ & 81 & \\
Total & 81 & & 275 & $82 \%$ & 59 & $18 \%$ & 334 & \\
\hline
\end{tabular}


Voyons à présent les effets de fréquence selon la forme verbale employée: dans le tableau (152) figurent les formes verbales le plus fréquemment niées du corpus. Nous avons intégré dans le tableau toute forme verbale qui apparait plus de 10 fois sous forme négative ainsi que les deux formes contractées $\left[\int \varepsilon\right]$ pour je sais et [ $\left.\int \mathrm{u} i\right]$ pour je suis, même si cette dernière ne montre que 5 occurrences négatives $(c f$. annexe XII pour une liste des toutes les constructions négatives contenant une forme contractée).

(152) \pm ne selon la fréquence de la forme verbale ${ }^{124}$

\begin{tabular}{|c|c|c|c|c|c|c|c|}
\hline & Forme verbale & $-n e$ & $\%$ & $+n e$ & $\%$ & Total & $\chi^{2}$ \\
\hline \multirow{7}{*}{$>10$ occurrences } & [a] $a$ & 13 & $87 \%$ & 2 & $13 \%$ & 15 & \multirow{7}{*}{$\begin{array}{l}p=0,002 \\
\text { (formes } \\
\text { fréquentes et } \\
\text { contractées } \\
\text { vs. les autres } \\
\text { formes) }\end{array}$} \\
\hline & [a] $a+\mathrm{X}$ & 12 & $71 \%$ & 5 & $29 \%$ & 17 & \\
\hline & {$[\mathrm{e}] /[\varepsilon]$ est } & 50 & $86 \%$ & 8 & $14 \%$ & 58 & \\
\hline & {$[\mathrm{e}] /[\varepsilon]$ est $+\mathrm{X}$} & 20 & $95 \%$ & 1 & $5 \%$ & 21 & \\
\hline & {$[$ ave/E] avai (en)t $(+\mathrm{X})$} & 9 & $90 \%$ & 1 & $10 \%$ & 10 & \\
\hline & [e/ctc] étai $(e n) t(+\mathrm{X})$ & 13 & $100 \%$ & 0 & $0 \%$ & 13 & \\
\hline & {$[\mathrm{se} / \varepsilon]$ sais/t } & 26 & $81 \%$ & 6 & $19 \%$ & 32 & \\
\hline \multirow{2}{*}{$\begin{array}{l}\text { Formes } \\
\text { contractées }\end{array}$} & {$\left[\int \varepsilon\right]$ je sais } & 9 & $100 \%$ & 0 & $0 \%$ & 9 & \\
\hline & [كчi] je suis & 5 & $100 \%$ & 0 & $0 \%$ & 5 & \\
\hline Total & & 157 & $87 \%$ & 23 & $13 \%$ & 180 & \\
\hline
\end{tabular}

Les formes très fréquentes et contractées manifestent effectivement des valeurs significativement inférieures de $+n e$ par rapport aux formes restantes.

124 Le calcul se base sur la réalisation phonétique de la forme verbale ( $c f$. section 3.7 pour le système de transcription). Pour cette raison, nous trouvons, d'une part, des réalisations alternatives pour une même forme verbale, comme par exemple les réalisations [e] et [ $\varepsilon$ ] pour est, et d'autre part, plusieurs formes homophones d'un paradigme verbal sous une seule forme, comme par exemple avait et avaient, qui peuvent correspondre à la forme phonologique [ave/c]. Par forme contractée nous désignons les complexes inséparables formés par un clitique sujet et un verbe fini $\left(\mathrm{Cl}_{\mathrm{s}}+\mathrm{V}_{\text {fin }}\right)$ qui ne permettent aucune insertion d'un clitique ultérieur, indépendamment du fait qu'il s'agisse d'un clitique de négation

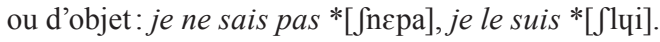


Par conséquent, nous retenons, de manière générale, les tendances suivantes pour les verbes lexicaux avec des fréquences entre 1 et 200 occurrences dans nos données: le grand groupe des verbes rares provoque tendanciellement la réalisation de $n e$, tandis que les quelques verbes très fréquents sont moins nombreux et favorisent plutôt l'omission de ne. Au niveau de la fréquence des formes verbales, ce sont les séquences contractées et presque lexicalisées comportant une variante clitique monosegmentale qui excluent la réalisation de $n e$.

Bien évidemment, les taux de \pm ne pourraient varier non seulement en fonction de la fréquence du verbe, mais également en fonction du temps et du mode de celui-ci. Même si les études précédentes sur $\pm n e$ ont montré que les temps et modes verbaux ne provoquent pas de différences considérables ( $c f$. section 2.2.5.5), nous testerons, par la suite, leurs influences dans notre corpus. Dans le tableau (153) figurent, en ordre décroissant selon le nombre d'occurrences, les temps et les modes verbaux employés dans le corpus et les taux de réalisation de ne correspondants.

(153) \pm ne selon le temps et le mode verbal

\begin{tabular}{|lrrrrrr|}
\hline Temps verbal & \multicolumn{1}{c}{-ne } & \multicolumn{1}{c}{$\%$} & $+n e$ & $\%$ & total & $\chi^{2}$ \\
\hline Indicatif présent & 188 & $80 \%$ & 47 & $20 \%$ & 235 & $p=0,351$ \\
Indicatif passé composé & 34 & $83 \%$ & 7 & $17 \%$ & 41 & \\
Indicatif imparfait & 30 & $94 \%$ & 2 & $6 \%$ & 32 & \\
Indicatif futur périphrastique & 9 & $100 \%$ & 0 & $0 \%$ & 9 & \\
Conditionnel présent & 6 & $86 \%$ & 1 & $14 \%$ & 7 & \\
Impératif & 2 & $67 \%$ & 1 & $33 \%$ & 3 & \\
Indicatif présent passif & 2 & $100 \%$ & 0 & $0 \%$ & 2 & \\
Conditionnel passé I & 1 & $100 \%$ & 0 & $0 \%$ & 1 & \\
Indicatif futur simple & 0 & $0 \%$ & 1 & $100 \%$ & 1 & \\
Indicatif plus-que-parfait & 1 & $100 \%$ & 0 & $0 \%$ & 1 & \\
Indicatif présent / impératif? & 1 & $100 \%$ & 0 & $0 \%$ & 1 & \\
Subjonctif présent & 1 & $100 \%$ & 0 & $0 \%$ & 1 & \\
Total & 275 & $82 \%$ & 59 & $18 \%$ & 334 & \\
\hline
\end{tabular}


Dans les premières lignes du tableau figurent les temps verbaux les plus fréquents du corpus. Il s'agit des formes de l'indicatif: le présent $(20 \%+n e)$, le passé composé $(17 \%+n e)$, l'imparfait $(6 \%+n e)$ et le futur périphrastique $(0 \%+n e)$. Tandis que les taux du présent et du passé composé reflètent à peu près la moyenne du corpus, l'imparfait et le futur périphrastique manifestent des pourcentages nettement plus bas. Le taux de réalisation dans les constructions impératives se situe, avec $27 \%$ +ne, à un niveau intermédiaire, légèrement supérieur à la moyenne du corpus. Ceci dément clairement la règle proposé par Ashby (1981: 680), «the imperative retains ne categorically», vu que deux phrases impératives sur trois sont réalisées sans ne ( $c f$. section 5.2.4.6 pour une discussion approfondie de l'impératif négatif).

(154) $\pm n e$ dans les constructions impératives négatives

a. alors parlez pas (1568)

b. ne repassez pas jamais $(2590)^{125}$

c. fais pas cette tête (1090)

D'autres temps ou modes verbaux ne sont employés qu'une ou deux fois dans des constructions négatives et leurs taux de $100 \%$ ou de $0 \%+n e$ ne se prêtent donc à aucune interprétation généralisable. Toutefois, même en omettant les cas en question, les temps et modes verbaux ne produisent aucun effet statistiquement significatif. Les différences observées quant à ce facteur pourraient donc être purement aléatoires.

Avant de conclure la section dédiée à l'influence du verbe sur la réalisation de $n e$, nous allons encore tester l'influence de différents types de verbes selon une classification purement sémantique et selon deux classifications sémantico-syntaxiques. Dans le tableau (155), nous distinguons entre les verbes d'état (être, aimer, dormir etc.), les verbes d'action (aller, demander, dire etc.) et les verbes de procès (tomber, devenir, changer etc.) (d'après Jones ${ }^{3} 2007$ : 51). Dans le tableau (156), nous faisons la différence entre avoir/être, les verbes

125 La discussion de cet exemple dans la section 5.1.4 montrera que la présence de ne est ici avant tout explicable par le contexte pragmatique. 
modaux (pouvoir, devoir, savoir etc.) et les verbes lexicaux. Finalement, dans le tableau (157) figurent les verbes classés selon leur valence respective telle qu'elle est indiquée par Busse/Dubost ( $\left.{ }^{2} 1983\right)$.

(155) \pm ne selon la sémantique du verbe

\begin{tabular}{|lrcrcrc|}
\hline & $-n e$ & $\%$ & $+n e$ & $\%$ & Total & $\chi^{2}$ \\
\hline Verbes d'état & 202 & $86 \%$ & 33 & $14 \%$ & 235 & $p=0,017$ \\
Verbes d'action & 56 & $72 \%$ & 22 & $28 \%$ & 78 & \\
Verbes de procès & 17 & $81 \%$ & 4 & $19 \%$ & 21 & \\
Total & 275 & $82 \%$ & 59 & $18 \%$ & 334 & \\
\hline
\end{tabular}

(156) $\pm n e$ selon la classe sémantico-syntaxique du verbe

\begin{tabular}{|lrccccc|}
\hline & $-n e$ & $\%$ & $+n e$ & $\%$ & Total & $\chi^{2}$ \\
\hline avoirlêtre & 92 & $84 \%$ & 17 & $16 \%$ & 109 & $p=0,036$ \\
Verbes modaux & 81 & $89 \%$ & 10 & $11 \%$ & 91 & \\
Verbes lexicaux & 102 & $76 \%$ & 32 & $24 \%$ & 134 & \\
Total & 275 & $82 \%$ & 59 & $18 \%$ & 334 & \\
\hline
\end{tabular}

(157) \pm ne selon la valence ${ }^{126}$ du verbe

\begin{tabular}{|llrrrrrr|}
\hline $\begin{array}{l}\text { Valence verbale } \\
\text { (nombre de verbes) }\end{array}$ & Exemple & -ne & $\%$ & ne & $\%$ & Total & $\chi^{2}$ \\
\hline Avalent (1) & falloir & 3 & $100 \%$ & 0 & $0 \%$ & 3 & $p=0,174$ \\
Monovalent (2) & dormir & 2 & $67 \%$ & 1 & $33 \%$ & 3 & \\
Bi-valent (17) & déranger & 24 & $73 \%$ & 9 & $27 \%$ & 33 & \\
Tri-valent (7) & convaincre & 10 & $91 \%$ & 1 & $9 \%$ & 11 \\
Mono-bi-valent (4) & arriver & 6 & $100 \%$ & 0 & $0 \%$ & 6 & \\
Bi-tri-valent (11) & aider & 11 & $65 \%$ & 6 & $35 \%$ & 17 \\
Mono-bi-tri-valent (3) & parler & 6 & $100 \%$ & 0 & $0 \%$ & 6 & \\
Autres/multiples sous- & aller & 213 & $84 \%$ & 42 & $16 \%$ & 255 \\
catégorisations (36) & & 275 & $82 \%$ & 59 & $18 \%$ & 334 & \\
Total (81 verbes) & & & & & & & \\
\hline
\end{tabular}

126 Notre classification suit celle de Busse/Dubost (21983: 44) et dépend, dans les cas de doute, de l'emploi concret dans notre corpus. 
Sans observer de différences considérables ou vraiment significatives entre les différentes classes sémantiques et sémantico-syntaxiques en (155) et (156), nous notons que ce sont les verbes d'action $(28 \%+n e)$ et tout le groupe des verbes lexicaux $(24 \%+n e)$ qui provoquent les taux de $n e$ les plus élevés. Les verbes de procès $(19 \%+n e)$ et avoirlêtre $(16 \%+n e)$ se situent au milieu, et les verbes d'état $(14 \%+n e)$ et modaux $(11 \%+n e)$ manifestent les taux les plus bas de ne. Dans le tableau (157), la première colonne spécifie la valence du verbe et le nombre d'occurrences des verbes (apparaissant à l'intérieur d'une construction négative dans le corpus) qui appartiennent à cette catégorie. Les taux de réalisation de $n e$ selon la valence du verbe se situent entre $0 \%+n e$ (provoqué par les verbes mono-, bi- et trivalents) et 35\% +ne (avec les verbes bi- et trivalents). Il n'y a aucune tendance claire qui suggérerait que la valence lexicale (donc le nombre d'arguments potentiels) soit liée de manière quelconque à la réalisation du ne, vu que les valeurs extrêmes dans le tableau (157) correspondent à trois groupes de verbes qui sont très similaires.

Nous avons vu dans cette section que ni les temps et les modes verbaux, ni les classes sémantiques ou syntaxiques des verbes n'exercent une influence notable sur \pm ne dans notre corpus. La fréquence des différents verbes et les 'séquences préformées' ( $c f$. Moreau 1986) qui excluent l'emploi de ne, comme je sais [ $\left.\int \varepsilon\right]$ ou je suis, [ $\left.\int \mathrm{yi}\right]$ avec une négation semblent les seuls facteurs pertinents dans ce contexte.

\subsubsection{Le type de phrase}

\subsubsection{L'opposition entre les principales et les subordonnées}

Ashby (1976: 125, 1981: 678) et Sturm (1981: 62) ont découvert que la réalisation de ne est plus fréquente dans les subordonnées que dans les principales ( $c f$. section 2.2.5.6). Les données en (158) montrent que cette tendance significative se trouve également dans notre corpus. 
(158) \pm ne selon le type de phrase

a. Pourcentages

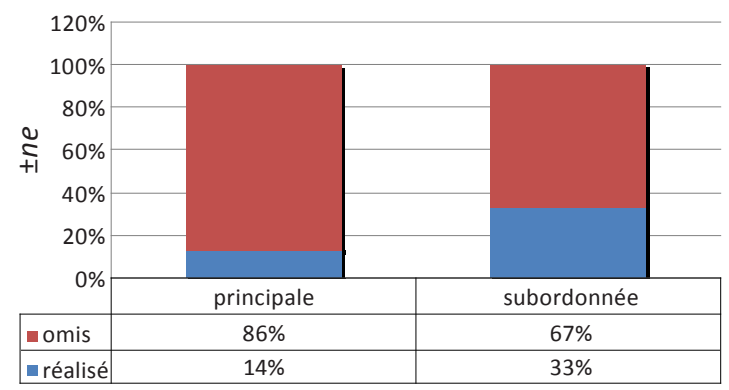

b. Données brutes

\begin{tabular}{|lrrrrrr|}
\hline & \multicolumn{2}{c}{$-n e$} & \multicolumn{1}{c}{$+n e$} & Total & $\chi^{2}$ \\
\hline Non identifiable & 3 & $100 \%$ & 0 & $0 \%$ & 3 & $p<0,000$ \\
Principale & 224 & $86 \%$ & 35 & $14 \%$ & 259 & \\
Subordonnée & 48 & $67 \%$ & 24 & $33 \%$ & 72 & \\
Total & 275 & $82 \%$ & 59 & $18 \%$ & 334 & \\
\hline
\end{tabular}

Nous notons également une différence (cette fois-ci, non-significative) à l'intérieur du groupe des subordonnées. Comme dans les études d'Ashby (1976, 1981), les relatives montrent un taux de ne supérieur aux autres types de subordonnées:

(159) \pm ne selon le type de subordonnée

a. Pourcentages

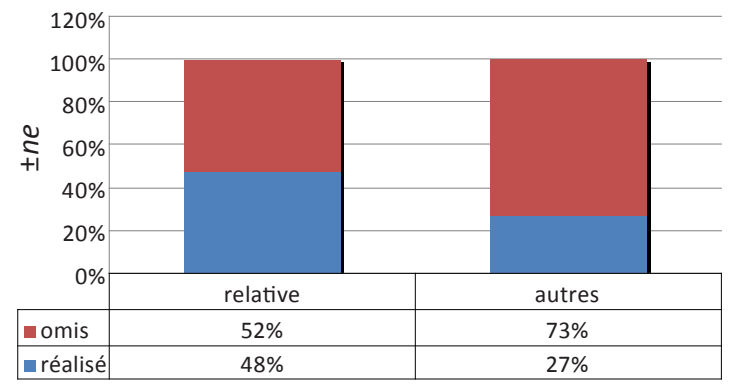


b. Données brutes

\begin{tabular}{|lrlllll|}
\hline & $-n e$ & & & $+n e$ & $\chi^{2}$ \\
\hline Relatives & 11 & $52 \%$ & 10 & $48 \%$ & 21 & $p=0,099$ \\
Autres subordonnées & 37 & $73 \%$ & 14 & $27 \%$ & 51 & \\
& 48 & $67 \%$ & 24 & $33 \%$ & 72 & \\
\hline
\end{tabular}

La discussion en section 5.2.4.2 montrera que l'influence du type de phrase s'inscrit dans une explication prosodique générale de l'absence et présence de $n e$.

\subsubsection{Les constructions interrogatives et clivées}

Les constructions négatives spécifiques, comme les interrogatives et les clivées, sont plutôt rares dans le corpus et ne se prêtent à aucune interprétation quantitative. Toutefois, nous tenons à retenir que la seule instance de ne dans une interrogative niée (160)a se trouve dans une question à l'inversion, $c f$. (160)a. Par contre, les 15 questions niées restantes (majoritairement des questions intonatives) ne manifestent aucune instance de ne, comme les exemples (160)b-d le montrent.

(160) \pm ne dans les constructions interrogatives

a. ce n'est(-il) pas la Dalmatie? (1399) 127

b. on peut pas faire les bruits des animaux? (0656)

c. est-ce qu'avant il y avait pas déjà deux codes un oral et un écrit (0059)

d. pourquoi ils viennent pas nous chercher? (1278)

De la même façon, les trois constructions clivées niées du corpus, qui figurent en (161), ne contiennent aucun ne.

(161) $\pm n e$ dans les constructions clivées

a. il y a que quand je faisais de l'improvisation que je la regardais (1934)

b. et il y a avait que cinq minutes qui avaient passé (1940)

c. c'est pas $[\mathrm{NomPr}]$ qui est en train de parler (1996)

127 Le clitique $i l$ figure entre parenthèses parce que l'enregistrement ne permet pas de discerner s'il y a réellement une inversion du clitique ou non ( $c f$. section 3.7). 


\subsubsection{Le contexte phonétique}

$\mathrm{Vu}$ que la particule proclitique ne est souvent directement adjacente au verbe fini ou à l'auxiliaire, nous supposons que la réalisation phonétique de l'attaque verbale a une certaine influence sur sa réalisation. En (162) figurent les taux de réalisation de ne selon le phonème initial de la forme verbale respective.

(162) \pm ne selon l'attaque de la forme verbale

a. Attaque vocalique vs. consonantique

\begin{tabular}{|lcccccc|}
\hline Attaque & $-n e$ & $\%$ & $+n e$ & $\%$ & Total & $\chi^{2}$ \\
\hline Vocalique & 132 & $84 \%$ & 25 & $16 \%$ & 157 & $p=0,432$ \\
Consonantique & 143 & $81 \%$ & 34 & $19 \%$ & 177 & \\
Total & 275 & $82 \%$ & 59 & $18 \%$ & 334 & \\
\hline
\end{tabular}

b. Attaques détaillées

\begin{tabular}{|c|c|c|c|c|c|c|c|c|}
\hline \multicolumn{3}{|l|}{ Attaque verbale } & $-n e$ & $\%$ & $+n e$ & $\%$ & Total & $\chi^{2}$ \\
\hline \multirow[t]{7}{*}{ Vocalique } & \multirow[t]{5}{*}{ non-nasales } & {$[\mathrm{a}]$} & 39 & $81 \%$ & 9 & $19 \%$ & 48 & \multirow[t]{19}{*}{$p=0,319$} \\
\hline & & {$[\mathrm{e} / \varepsilon]$} & 88 & $87 \%$ & 13 & $13 \%$ & 101 & \\
\hline & & {$[\mathrm{y}]$} & 1 & $100 \%$ & 0 & $0 \%$ & 1 & \\
\hline & & {$[\mathrm{u}]$} & 1 & $100 \%$ & 0 & $0 \%$ & 1 & \\
\hline & & [0] & 1 & $50 \%$ & 1 & $50 \%$ & 2 & \\
\hline & \multirow[t]{2}{*}{ nasales } & {$[\tilde{\varepsilon}]$} & 1 & $50 \%$ & 1 & $50 \%$ & 2 & \\
\hline & & {$[\tilde{\jmath}]$} & 1 & $50 \%$ & 1 & $50 \%$ & 2 & \\
\hline \multirow[t]{11}{*}{ Consonantique } & \multirow[t]{2}{*}{ bilabiales } & [p] & 24 & $89 \%$ & 3 & $11 \%$ & 27 & \\
\hline & & {$[\mathrm{m}]$} & 4 & $50 \%$ & 4 & $50 \%$ & 8 & \\
\hline & \multirow[t]{2}{*}{ labio-dentales } & {$[\mathrm{f}]$} & 7 & $88 \%$ & 1 & $13 \%$ & 8 & \\
\hline & & [v] & 27 & $84 \%$ & 5 & $16 \%$ & 32 & \\
\hline & \multirow[t]{3}{*}{ alvéolaires } & {$[\mathrm{t}]$} & 1 & $50 \%$ & 1 & $50 \%$ & 2 & \\
\hline & & [d] & 4 & $67 \%$ & 2 & $33 \%$ & 6 & \\
\hline & & {$[\mathrm{s}]$} & 39 & $78 \%$ & 11 & $22 \%$ & 50 & \\
\hline & post-alvéolaires & {$\left[\int\right]^{128}$} & 18 & $95 \%$ & 1 & $5 \%$ & 19 & \\
\hline & \multirow[t]{2}{*}{ vélaires } & [3] & 0 & $0 \%$ & 2 & $100 \%$ & 2 & \\
\hline & & {$[\mathrm{k}]$} & 9 & $100 \%$ & 0 & $0 \%$ & 9 & \\
\hline & uvulaires & [в] & 10 & $71 \%$ & 4 & $29 \%$ & 14 & \\
\hline Total & & & 275 & $82 \%$ & 59 & $18 \%$ & 334 & \\
\hline
\end{tabular}

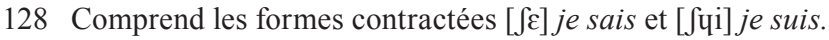


Contrairement à ce que l'on pourrait attendre, ni l'opposition entre une attaque vocalique ou consonantique, ni les attaques détaillées ne produisent des différences notables ou significatives.

Étant donné que le contexte droit seul ne produit aucune variation significative de $\pm n e$, considérons maintenant le contexte phonétique dans son intégralité. Dans le tableau (163) figure une vue d'ensemble des contextes phonétiques gauches et droits de la variable \pm ne dans le corpus.

Nous distinguons six types de contextes: il s'agit des quatre combinaisons possibles de voyelles et de consonnes, d'un contexte postpausal, qui, dans notre corpus, n'apparaît que suivi par des consonnes (\#_C), et des complexes clitique+verbe quasiment agglutinés, comme [ste] pour c'étai(en)t, [ja] pour (il) y a et [ $\left.\int \mathrm{e}\right]$ ou $\left[\int \varepsilon\right]$ pour je sais (cf. aussi section 4.4.4 pour une discussion de ces cas).

(163) Les contextes phonétiques de $\pm n e$

\begin{tabular}{|lrrrrrr|}
\hline Contexte phonétique & $-n e$ & $\%$ & $+n e$ & $\%$ & Total & $\chi^{2}$ \\
\hline V_V & 6 & $40 \%$ & 9 & $60 \%$ & 15 & $p=0,000$ \\
V_C & 74 & $80 \%$ & 18 & $20 \%$ & 92 & \\
C_C & 69 & $75 \%$ & 23 & $25 \%$ & 92 & \\
C_V & 98 & $93 \%$ & 7 & $7 \%$ & 105 & \\
\#_C & 1 & $33 \%$ & 2 & $67 \%$ & 3 & \\
Forme contractée & 26 & $100 \%$ & 0 & $0 \%$ & 26 & \\
Cas exclu - position de $n$ ne n'est pas claire & 1 & & 0 & & 1 & \\
Total & 275 & $82 \%$ & 59 & $18 \%$ & 334 & \\
\hline
\end{tabular}

Contrairement à l'attaque verbale, les différences quant à la réalisation de ne entre les divers contextes phonétiques sont extrêmement significatives, et le nombre de cas par groupe est, sauf dans les contextes postpausals, suffisamment élevé pour permettre des conclusions solides: contrairement à la tendance générale à l'omission du ne qui se manifeste dans le corpus, dans le contexte V_V, $60 \%$ des ne ont été réalisés. Dans le contexte \#_C, la réalisation de ne semble également la variante préférée, même si la base empirique (trois cas au total) n'est pas assez solide. Dans ce contexte, nous citons Ashby (1981: 677), qui trouve 
des tendances identiques quant à la réalisation de $n e:$ «It is likely to be retained postpausally [...] and in intervocalic position provided one of the vowels is nasal». À l'inverse, les contextes restants manifestent des valeurs relativement, ( $c f$. V_C 20\% +ne et C_C 25\% +ne) voire extrêmement basses ( $\left.c f . \mathrm{C}_{-} \mathrm{V} 7 \%+n e\right)$.

Dans ce qui suit, nous allons vérifier, grâce à une analyse plus détaillée, l'affirmation d'Ashby (1981) selon laquelle l'une des voyelles devrait être une nasale pour provoquer la réalisation de ne. En (164) figurent les quatre combinaisons de voyelles et consonnes et le contexte post-pausal, sous-classifiés, cette fois-ci, selon voyelles nasales (V) et non nasales $(\mathrm{V})$, et consonnes sourdes $\left(\mathrm{C}_{-}\right.$sou) et sonores $\left(\mathrm{C}_{-} \text {son }\right)^{129}$.

(164) \pm ne selon l'environnement phonétique ${ }^{130}$

a. Pourcentages

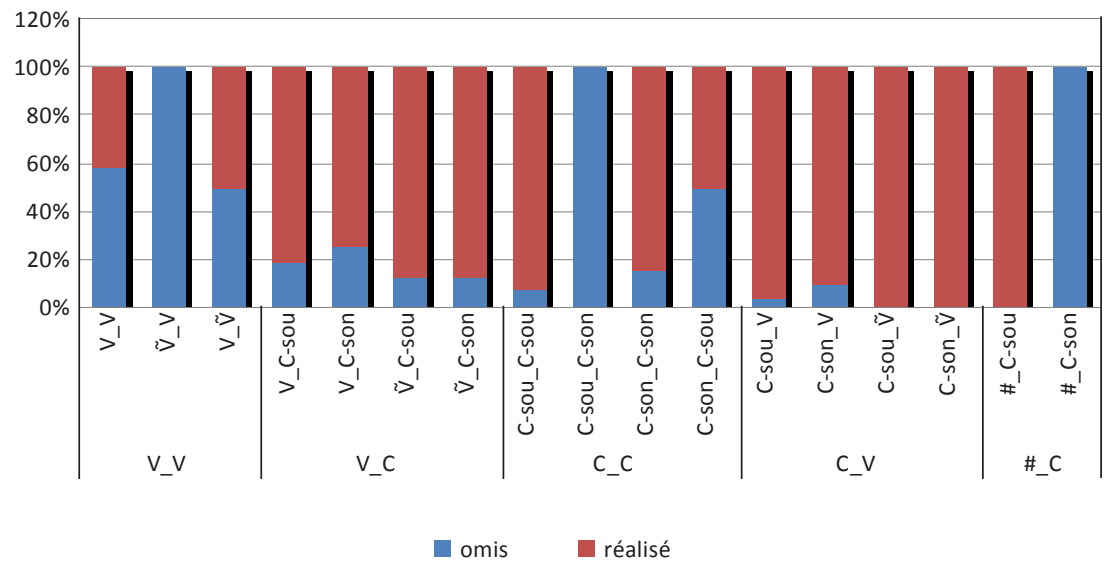

129 Suivant la hiérarchie sonore adoptée pour le français par Pustka (2011: 113), nous considérons comme C-sonores: [m], [v], [1], [r], [d], [3] et [z]. Le son [j], la seule glide qui apparaît en adjacence à ne, est également traitée ici comme une consonne sonore. Comme C-sourdes, nous comptons: [s], [S], [f], [k], [p] et [t].

$130 C f$. l'annexe XIII pour les taux exacts de $\pm n e$ dans tous les contextes phonétiques. 
b. Données brutes

\begin{tabular}{|c|c|c|c|c|c|c|c|}
\hline \multicolumn{2}{|c|}{ Environnement phonétique } & \multirow{2}{*}{$\begin{array}{r}-n e \\
5\end{array}$} & \multirow{2}{*}{$\begin{array}{l}\% \\
42 \%\end{array}$} & \multirow{2}{*}{$\begin{array}{r}+n e \\
7\end{array}$} & \multirow{2}{*}{$\begin{array}{l}\% \\
58 \%\end{array}$} & \multirow{2}{*}{$\begin{array}{r}\text { Total } \\
12\end{array}$} & \multirow[b]{2}{*}{$\leftrightarrow$} \\
\hline V_V & $\mathrm{V}_{-} \mathrm{V}$ & & & & & & \\
\hline & $\tilde{V}_{-} \mathrm{V}$ & 0 & $0 \%$ & 1 & $100 \%$ & 1 & $\leftrightarrow$ \\
\hline & V_V & 1 & $50 \%$ & 1 & $50 \%$ & 2 & $\leftrightarrow$ \\
\hline \multirow[t]{4}{*}{ V_C } & V_C-sourde & 30 & $81 \%$ & 7 & $19 \%$ & 37 & $\downarrow$ \\
\hline & V_C-sonore & 23 & $74 \%$ & 8 & $26 \%$ & 31 & $\downarrow$ \\
\hline & $\tilde{V} \_C$-sourde & 14 & $88 \%$ & 2 & $13 \%$ & 16 & $\downarrow$ \\
\hline & $\tilde{\mathrm{V}} \_$C-sonore & 7 & $88 \%$ & 1 & $13 \%$ & 8 & $\downarrow$ \\
\hline \multirow[t]{4}{*}{ C_C } & C-sourde_C-sourde & 24 & $92 \%$ & 2 & $8 \%$ & 26 & $\leftrightarrow$ \\
\hline & C-sourde_C-sonore & 0 & $0 \%$ & 4 & $100 \%$ & 4 & $\uparrow$ \\
\hline & C-sonore_C-sonore & 36 & $84 \%$ & 7 & $16 \%$ & 43 & $\leftrightarrow$ \\
\hline & C-sonore_C-sourde & 10 & $50 \%$ & 10 & $50 \%$ & 20 & $\downarrow$ \\
\hline \multirow[t]{4}{*}{$\mathrm{C}_{-} \mathrm{V}$} & C-sourde_V & 48 & $96 \%$ & 2 & $4 \%$ & 50 & $\uparrow$ \\
\hline & C-sonore_V & 45 & $90 \%$ & 5 & $10 \%$ & 50 & $\uparrow$ \\
\hline & C-sourde_ $\tilde{V}$ & 1 & $100 \%$ & 0 & $0 \%$ & 1 & $\uparrow$ \\
\hline & C-sonore_ $\tilde{V}$ & 3 & $100 \%$ & 0 & $0 \%$ & 3 & $\uparrow$ \\
\hline \multirow[t]{4}{*}{ \#_C } & \#_C-sourde & 1 & $100 \%$ & 0 & $0 \%$ & 1 & \\
\hline & \#_C-sonore & 0 & $0 \%$ & 2 & $100 \%$ & 2 & \\
\hline & Forme contractée & 26 & $100 \%$ & 0 & $0 \%$ & 26 & \\
\hline & Exclu - position de ne n'est pas claire & 1 & - & 0 & - & 1 & \\
\hline Total & & 275 & & 59 & & 334 & \\
\hline
\end{tabular}

Le diagramme (164)a semble confirmer la deuxième observation d'Ashby (1981: 677) selon laquelle ce sont en particulier les voyelles nasales qui provoquent à la réalisation de ne. Toutefois, la valeur de $100 \%+n e$ pour le contexte $\tilde{V}_{-} \mathrm{V}$ n'est fondée que sur un seul cas et ne constitue donc aucune base empirique valide ${ }^{131}$. De la même façon, les deux autres valeurs de $100 \%$ +ne ne se basent que sur très peu de cas: pour le contexte C-sourde_C-sonore nous n'avons compté que quatre occurrences et pour le contexte postpausal \#_C-sonore, seulement deux.

131 Ce problème naît du fait que la plupart de ces contextes ont été exclus de l'analyse en raison de la liaison obligatoire entre les voyelles nasales et non-nasales adjacentes et appartenant à un même groupe rythmique: on (n') a pas [õnapas] (cf. section 3.8). 
Quoi qu'il en soit, nous pouvons tout de même observer quelques tendances intéressantes en considérant la structure syllabique et le profil de sonorité provoqués par les contextes phonétiques manifestant une réalisation de ne de $50 \%$ +ne ou plus élevée:

(165) Les structures syllabiques des contextes $+n e$

\begin{tabular}{|c|c|c|c|c|c|c|}
\hline Contexte & $-n e$ & $+n e$ & Syllabes & & Sonorité & Exemple \\
\hline $\mathrm{V}_{-} \mathrm{V}$ & $42 \%$ & $58 \%$ & V.nV & V.CV & $\uparrow \uparrow \downarrow \uparrow$ & [3ə.nع.pa.ã.vi] (0108) \\
\hline$\tilde{V}_{-} \mathrm{V}$ & $0 \%$ & $100 \%$ & $\tilde{\mathrm{V}} . \mathrm{nV}$ & V.CV & $\uparrow \downarrow \uparrow$ & 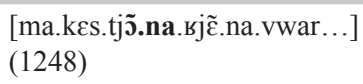 \\
\hline $\mathrm{V}_{-} \tilde{\mathrm{V}}$ & $50 \%$ & $50 \%$ & V.ñ & V.CV & $\uparrow \downarrow \uparrow$ & [ki.ñ̃.pa.ak.sep.te...] (2124) \\
\hline C-sou_C-son & $0 \%$ & $100 \%$ & C.nə.C & C.CV.C & $\uparrow \downarrow \uparrow \downarrow$ & $\begin{array}{l}\text { [s̃̃.vø.də.sa.zes.nə.lui.a.pa] } \\
(0342)\end{array}$ \\
\hline C-son_C-sou & $50 \%$ & $50 \%$ & C.nə.C & C.CV.C & $\uparrow \downarrow \uparrow \downarrow$ & $\begin{array}{l}\text { [la.fuaz.nə.jãz.pa.sõ.sãs] } \\
\text { (0418) }\end{array}$ \\
\hline
\end{tabular}

Entre deux voyelles, l'insertion de [n] évite un hiatus. Le schwa étant élidé, la séquence syllabique produite contient celle universellement privilégiée, à savoir (V.)CV, et le profil de sonorité forme un tour à tour parfait $\uparrow \downarrow \uparrow$ entre les segments sonores et sourds. Curieusement, les séquences $\mathrm{C} \_\mathrm{C}$ peuvent être analysées selon le même schéma, pourvu que le schwa du ne soit réalisé. Apparemment, les différences de sonorité entre les deux consonnes adjacentes à ne privilégient sa réalisation avec un schwa, fait observable dans les exemples (166)a et (167)a. Par contre, l'absence du schwa dans ces contextes produirait des séquences presque imprononçables comme celles présentées en (166)b et (167)b.

(166) et que son vœu de sagesse ne lui a pas été profitable (0342)
a. [sa.zes.nə.lui]
b. ?[sa.zes.n.lyi]

(167) la phrase ne change pas son sens (0418)
a. [fваz.nə.jãz]
b. ?[ваz.n.fãz] 
Ainsi, dans les contextes qui le favorisent, $n(e)$ est toujours intégré dans une syllabe CV. La forme de syllabe pleine semble donc une précondition pour la stabilité de ne. En effet, le tableau (168) montre que la comparaison entre les cas qui 'conservent' ne à l'intérieur d'une syllabe pleine et les cas restants produit une différence extrêmement significative.

(168) \pm ne selon la structure syllabique

\begin{tabular}{|lrlrlrr|}
\hline Structure syllabique autour de $\pm n e$ & \multicolumn{1}{c}{$-n e$} & $\%$ & $+n e$ & $\%$ & Total & $\chi^{2}$ \\
\hline Syllabes CV & 16 & $39 \%$ & 25 & $61 \%$ & 41 & $p<0,000$ \\
D'autres syllabes & 232 & $87 \%$ & 34 & $13 \%$ & 266 & \\
\hline
\end{tabular}

\subsubsection{Conclusion intermédiaire: l'influence des facteurs linguistiques}

Nous avons donc démontré qu'au niveau linguistique ce sont avant tout les types de sujet et de phrase qui influencent la réalisation de $\pm n e$. L'influence pertinente sur \pm ne dérive donc en premier lieu du contexte gauche: si $\pm n e$ est précédé par un élément prosodiquement lourd, les possibilités de sa réalisation sont beaucoup plus élevées qu'avec un clitique ou dans une séquence de clitiques. Finalement, le contexte phonétique de la variable et la structure syllabique dans laquelle elle est intégrée semblent extrêmement pertinents: tandis que les syllabes pleines 'protègent' le ne, tous les autres types de syllabes favorisent son omission.

De même, dans le contexte droit, la fréquence des éléments peut jouer un rôle: avec la particule pas, employée deux fois plus souvent que l'ensemble des autres éléments négatifs postverbaux, la réalisation de ne est décidemment la plus basse. Néanmoins, les types de verbe et l'élément négatif non-clitique n'exercent une influence qu'à travers leurs fréquences respectives: les éléments rares favorisent la présence de ne et ceux fréquents son absence. Seul l'emploi des pronoms indéfinis négatifs comme personne et rien en tant que sujet peut provoquer la réalisation de ne, ce qui témoigne ici encore de la puissance du contexte gauche de la variable. 
Dans le but de comprendre l'importance du contexte gauche, il est nécessaire de tenir compte, tout d'abord, du fait que, dans le langage spontané, les éléments lourds en position de sujet sont considérablement plus rares que les éléments légers, comme nous allons montrer lors de l'analyse multifactorielle dans le chapitre suivant.

\subsection{Analyse multifactorielle}

\subsubsection{L'interaction entre \pm ne et une série de variables dites 'indépendantes'}

À ce stade de notre étude, une analyse multifactorielle nous permet de mieux discerner le poids respectif des influences individuelles sur $\pm n e$ et de détecter des liens pertinents entre celles-ci en ce qui concerne la variable en question. Pour effectuer cette analyse, nous utilisons le logiciel statistique et de programmation $R$ (cf. <http://www.r-project.org/> et Gries 2009 pour une introduction).

Les données sont préparées de la manière suivante: après l'exportation depuis la base de données sous format Excel, nous éliminons, dans un premier temps, tous les caractères spéciaux (accents, cédilles, espaces blancs, caractères API). Par la suite, nous ne gardons pour l'analyse multifactorielle que les variables qui sont spécifiées dans la base de données T-zéro pour chaque énoncé négatif, c'est-à-dire que les facteurs qui ne s'appliquent pas à tous les cas en question sont exclus. Ceci vaut, par exemple, pour la forme des sujets lexicaux, car seulement un sous-ensemble des énoncés négatifs possède un sujet lexical. Certaines lignes dans la colonne E en (169) restent par conséquent vides: 
(169) Les données brutes avant la transformation pour l'analyse en R

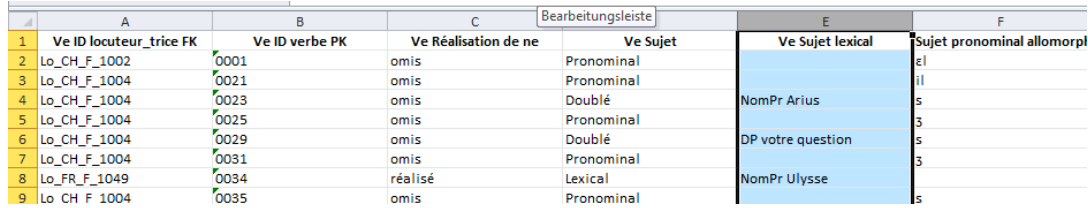

Afin de garder l'information des facteurs ainsi supprimés, certains sont transformés dans un format récurrent et lisible par R. La forme du sujet, lexical et pronominal, est transformée dans un format numérique scalaire en fonction du nombre des segments réalisés (p.ex. ça $[\mathrm{sa}]=2$, je $[3]=1$, Ulysse $[$ ylis] $=4)$. Cette transformation de la forme des sujets en échelle numérique en fonction de leur longueur correspond à l'observation faite dans les sections 2.2.5.1, 2.2.5.2 et 4.4.1, selon laquelle une distinction binaire entre les sujets pronominaux et lexicaux est insuffisante pour décrire l'influence du type de sujet sur la variation du $\pm n e$. Le degré de formalité de la situation est lui aussi transformé, de manière très simplifiée, en une échelle numérique: 1 = conversation informelle privée, 2 discussion informelle en cours et 3 = examen oral. Pour les facteurs type de sujet et situation de communication il existe donc deux classifications, dont l'une est catégorique et l'autre numérique.

En (170) figurent les variables que nous gardons pour l'analyse multifactorielle sous un format transformé.

(170) Les données brutes après la transformation pour l'analyse en $\mathrm{R}$

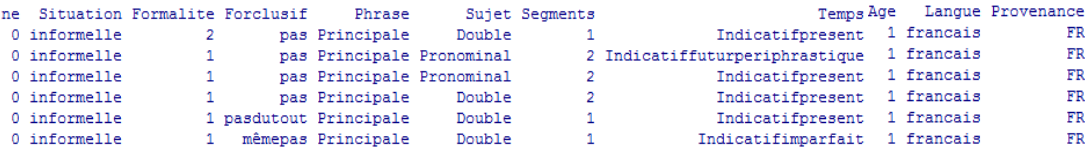

Lors de l'analyse multifactorielle, l'interaction entre huit variables (extra)linguistiques indépendantes et leur influence relative sur la variable dépendante \pm ne seront testées: 
(171) Les variables testées dans l'analyse multifactorielle

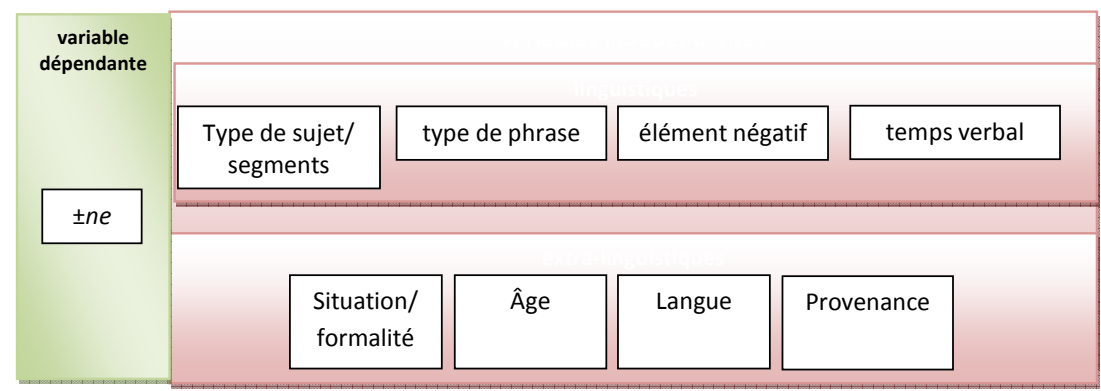

Afin de tester l'influence respective des variables et leurs interactions, nous utilisons la fonction du modèle linéaire généralisé (generalized linear model $\mathrm{glm}()$ ). Une formule exemplaire pour l'analyse multifactorielle de la variable dépendante \pm ne figure en (172): la variable dépendante est séparée des variables indépendantes par un tilde et les variables indépendantes sont séparées par des astérisques.

(172) multifac $<$-glm(ne $\sim$ Sujet * Forclusif* Phrase * Temps * Formalite * Age * Langue * Provenance)

Toutefois, dû aux particularités des variables (certains éléments négatifs ou temps verbaux n'apparaissent qu'une seule fois), une formule aussi complexe que celle en (172) ne produit aucun résultat valable. La formule est donc scindée en groupes de facteurs dont nous pouvons soupçonner une interaction pertinente pour l'explication de $\pm n e$.

Si nous passons maintenant aux résultats de l'analyse multifactorielle, la fonction summary() nous permet de voir les résultats sous forme de tableau. $\mathrm{R}$ se sert d'un code pour indiquer la signifiance des résultats : $p<0.000<* * *>; p<0.001=<* *>; p<0.01=<*>$. Nous rappelons qu'il y a une relation inversement proportionnelle entre la valeur $p$, qui indique la probabilité qu'il y ait une relation non-aléatoire entre deux variables, et la probabilité qu'une interdépendance existe entre les deux variables testées: plus $p$ est petit, plus une interdépendance est probable.

Compte tenu des résultats constatés lors de l'analyse descriptive, il n'est pas surprenant que, dans nos données, trois variables indépen- 
dantes apparaissent directement liées à la variable $\pm n e$ : le type de sujet $\left(p>0.000^{132}\right)$, le type de phrase $(p=0,024)$ et le degré de formalité de la situation $(p=0,011)$ :

(173) Les variables indépendantes ayant une influence sur \pm ne

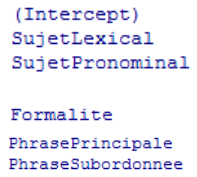

Les interactions pertinentes pour $\pm n e$ sont désignées par $\mathrm{R}$ à travers un double point entre les deux variantes en question ( $c f$. tableau (174)). $\mathrm{R}$ trouve également des liens entre le type de situation, d'un côté, et d'autres facteurs linguistiques de l'autre: le type de sujet $(p=0,047)$ et l'élément négatif non-clitique $(p=0,009)$; le type de phrase $(p<0,000)$ et le temps verbal $(p=0,002)$. Le seul facteur extralinguistique qui est lié de manière significative à la situation de communication est la provenance des locuteurs $(p<0,000)$.

(174) Les interactions pertinentes pour \pm ne entre la situation de communication et...

a. ...les facteurs linguistiques

\begin{tabular}{|c|c|c|c|c|c|c|}
\hline \multicolumn{7}{|c|}{ Estimate Std. Error t value $\operatorname{Pr}(>|t|)$} \\
\hline SujetLexical:Situationinformelle & $-7.018 e-02$ & 2.4 & $424 \mathrm{e}-01$ & -0.289 & 0.7724 & \\
\hline SujetPronominal: Situationinformelle & $-2.845 e-01$ & 1.4 & $430 e-01$ & -1.989 & 0.0476 & $*$ \\
\hline Forclusifpas: Situationinformelle & $5.918 \mathrm{e}$ & & $2.234 \mathrm{e}-01$ & 2.649 & 0.00851 & $* \star$ \\
\hline PhrasePrincipale:Situationinformelle & 0.41875 & & 0.08627 & 4.854 & $1.89 e-06$ & $\star * \star$ \\
\hline CempsIndicatifimparfait:Situationinformelle & 3.831 & & $1.249 \mathrm{e}-01$ & 3.068 & $0.00234 *$ & * * \\
\hline empsIndicatifpassecompose: Situationinformel & -3.00 & e-01 & $1.252 \mathrm{e}-01$ & -2.403 & $0.01686 *$ & \\
\hline
\end{tabular}

b. ...un facteur extra-linguistique

Formalite: ProvenanceFR $0.14973 \quad 0.03968 \quad 3.7730 .000192 * * *$

Finalement, pour les facteurs langue maternelle et âge, notre analyse multifactorielle n'a produit aucune interaction significative avecles variables testées: ces facteurs sont donc indépendants des autres variables.

$132 C f .<$ http://www.easysurf.cc/scintd.htm $>$ pour la conversion de la notation scientifique $3.83 \mathrm{e}-7$ en décimales. 
Pour conclure, nous observons en (175) que les variables indépendantes se séparent en différents groupes. Tout d'abord, une distinction entre les facteurs linguistiques et extralinguistiques, que nous avons déjà vue en (171), s'établit. Ensuite, il existe une séparation en trois colonnes: celle des influences directes, celle des influence indirectes et celle des variables sans influence.

(175) Résumé des résultats de l'analyse multifactorielle

\begin{tabular}{|c|c|c|c|c|}
\hline $\begin{array}{l}\text { Variable } \\
\text { dépendante }\end{array}$ & & Variables indépendantes & & \\
\hline $\pm n e$ & & influences primaires & $\begin{array}{l}\text { influences } \\
\text { secondaires }\end{array}$ & $\begin{array}{l}\text { sans } \\
\text { influence }\end{array}$ \\
\hline & linguistiques: & $\begin{array}{l}\text { type de sujet/segments } \\
(p<0.000 \text { et lié à la } \\
\text { situation: } p<0.005)\end{array}$ & $\begin{array}{l}\text { élément négatif } \\
\text { (lié à la situation } \\
\mathrm{p}<0.009)\end{array}$ & \\
\hline & & $\begin{array}{l}\text { type de phrase }(p=0,024 \\
\text { et lié à la situation: } \\
p<0.000)\end{array}$ & $\begin{array}{l}\text { temps verbal } \\
\text { (lié à la situation } \\
p<0,002)\end{array}$ & \\
\hline & $\begin{array}{l}\text { extra- } \\
\text { linguistiques: }\end{array}$ & $\begin{array}{l}\text { situation/formalité } \\
(p=0,011)\end{array}$ & $\begin{array}{l}\text { provenance } \\
\text { (liée à la situation } \\
p<0,000)\end{array}$ & âge / langue \\
\hline
\end{tabular}

Les influences directes, comme le type de sujet, le type de phrase (au niveau linguistique) et le type de situation (au niveau extralinguistique), sont liées de manière significative et directe à la variable dépendante $\pm n e$.

Bien sûr, le fait qu'il existe des relations très significatives entre $\pm n e$ et les influences primaires n'empêche pas que ces influences soient, de plus, en interaction avec d'autres variables dans le corpus. Au contraire, c'est précisément le fait de pouvoir détecter tout un réseau d'interdépendances entre les facteurs testés qui est l'avantage de l'analyse multifactorielle vis-à-vis de l'analyse descriptive (que nous avons effectuée dans les sections 4.3 et 4.4). Effectivement, le type de sujet et le type de phrase manifestent, au-delà de leur interaction avec $\pm n e$, également une interaction significative avec le type de situation. Par 
contre, les facteurs désignés comme influences secondaires en (175), à savoir l'élément négatif non-clitique, les temps et modes verbaux et la provenance géographique des locuteurs, ne manifestent aucune interaction directe avec la variable \pm ne en question, mais seulement une relation significative avec la situation de communication (qui est, elle, l'une des influences directes sur $\pm n e$ ).

Dans l'ensemble, nous pouvons donc présenter les interactions entre $\pm n e$ et les variables indépendantes sous forme d'un réseau:

(176) Les interactions entre $\pm n e$ et les variables indépendantes

Influences secondaires

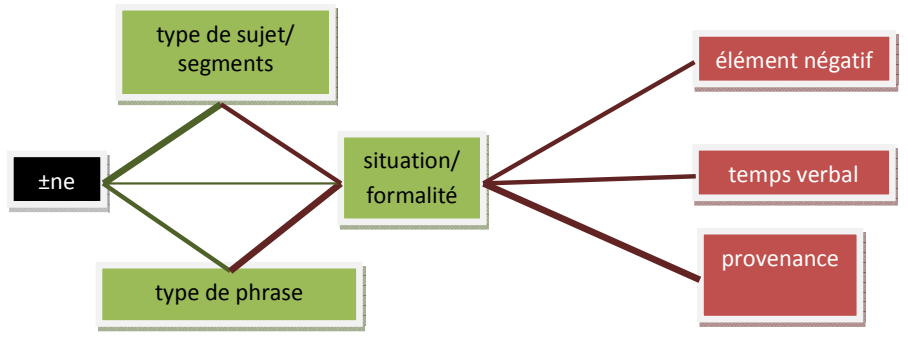

En (176), nous présentons les influences primaires et secondaires séparées selon leur nature linguistique ou extralinguistique. Les interactions entre les variables que nous avons détectées à l'aide de l'analyse multifactorielle sont affichées sous forme de lignes. Les lignes et les variables vertes désignent les influences directes sur $\pm n e$, et les facteurs et les lignes en rouge désignent les interdépendances pertinentes pour $\pm n e$ entre les variables indépendantes. L'épaisseur des lignes désigne le degré de signifiance statistique d'une interaction (plus une ligne est épaisse, plus l'interaction est significative).

Nous avons donc réussi, à l'aide de l'analyse multifactorielle, à scinder les influences pertinentes et directes sur \pm ne des influences indirectes et des facteurs sans influence. Ce sont le type de sujet, le type de phrase et la situation de communication qui ont une influence significative et directe sur $\pm n e$, tandis que l'élément négatif, le temps verbal et la provenance des locuteurs exercent une influence indirecte 
sur la variable en question, car ceux-ci sont liés à la situation de communication.

En effet, la situation de communication semble un facteur très puissant pour la variable $\pm n e$, et ceci non seulement parce qu'il exerce une influence directe (celle-ci est moins significative que celle du type de sujet ou de phrase), mais surtout parce qu'il est connecté à presque tous les autres facteurs testés. Il existe des interactions significatives entre la situation de communication et les influences linguistiques directes sur $\pm n e$, c'est-à-dire que le type de sujet et de phrase, l'élément négatif, le temps verbal et même la provenance des locuteurs semblent avoir une influence sur le comportement des locuteurs lors de différentes situations de communication (ce dernier phénomène a déjà été découvert lors de l'analyse descriptive en section 4.3.5 $)^{133}$. Le rôle de cette interaction entre la situation de communication et les facteurs linguistiques qui influencent $\pm n e$ est crucial pour la description théorique de cette variable et sera discuté en détail dans la section 5.3.

Dans l'ensemble, nous tenons à noter que, jusqu'ici, l'analyse multifactorielle ne nous permet que de détecter si des interactions pertinentes pour la variable dépendante existent entre les variables indépendantes, et ne nous renseigne aucunement sur la nature de ces liens. Il faut donc se poser deux questions:

i. L'interaction se manifeste-elle seulement par rapport à la variable $\pm n e$ ou s'agit-il d'une tendance discursive générale?

ii. S'agit-il d'une interdépendance mutuelle ou est-ce qu'un facteur est déterminé par l'autre?

133 Seuls les facteurs âge et langue maternelle apparaissent sans influence sur $\pm n e$ et sans connexion avec d'autres facteurs dans l'analyse multifactorielle. Dans le cas de la langue maternelle, ce résultat est en contradiction avec le résultat de l'analyse descriptive, $c f$. section 4.3.4. D’une manière générale, les résultats d'une analyse multifactorielle sont plus fiables que ceux d'une analyse descriptive, parce que la première tient en compte plusieurs facteurs simultanément. Néanmoins, vu que notre échantillon n'est pas précisément conçu pour tester l'importance éventuelle de la langue maternelle et que ce facteur a été découvert plutôt de façon inattendue lors de notre analyse, il semble donc nécessaire d'effectuer une recherche ciblée sur ce facteur. 
Dans le but de répondre à ces questions nous proposons, dans les sections suivantes, une analyse prudente des données de l'ensemble du corpus.

\subsubsection{L'interaction entre les variables dites 'indépendantes' dans le corpus entier}

Nous allons tester, par la suite, si les interactions détectées par rapport à la variable \pm ne se manifestent effectivement dans l'ensemble du corpus, ou bien si elles sont seulement pertinentes par rapport à la variable $\pm n e$ (cf. aussi la discussion en section 5.3). Avant de tester les corrélations en détail, la fonction summary() en R nous permet une vue d'ensemble de la répartition des variables pertinentes dans le corpus entier.

(177) Vue d'ensemble de la répartition des variables pertinentes dans T-zéro

a. Les influences primaires sur \pm ne

Le type de sujet/la longueur du sujet

\begin{tabular}{lrrl} 
& \multicolumn{2}{c}{ Sujet } & \multicolumn{2}{c}{ Segments } \\
Double & $: 268$ & Min. $: 0.000$ \\
imperatif & $:$ & 30 & 1st Qu. : 1.000 \\
Lexical & $:$ & 167 & Median $: 2.000$ \\
nonidentifiable: & 1 & Mean $: 2.051$ \\
Pronominal & $: 2033$ & Mrd Qu. : 2.000 \\
& & &
\end{tabular}

Le type de phrase

\begin{tabular}{lr}
\multicolumn{2}{c}{ Phrase } \\
Nonidentifiable: 24 \\
Principale $\quad 1826$ \\
Subordonnee $\quad: 649$
\end{tabular}

Le type de situation/le degré de formalité de la situation

\begin{tabular}{cl} 
Situation & \multicolumn{2}{c}{ Formalite } \\
formelle :1232 & Min. $: 1.000$ \\
informelle:1267 & 1st Qu. $: 1.000$ \\
& Median $: 2.000$ \\
& Mean $: 2.063$ \\
& 3rd Qu. $: 3.000$ \\
& Max. $: 3.000$
\end{tabular}


b. Les influences secondaires sur \pm ne

\begin{tabular}{|c|c|c|c|c|c|}
\hline Ten & nps & Provenance & \multicolumn{3}{|c|}{ Forclusif } \\
\hline Indicatifpresent & $: 1710$ & $\mathrm{CH}: 1268$ & \multicolumn{3}{|c|}{$: 2145$} \\
\hline Indicatifpassecompose & : 296 & \multirow{6}{*}{ FR: 1231} & pas & : & 255 \\
\hline Indicatifimparfait & : 171 & & que & : & 18 \\
\hline Indicatiffuturperiphrastique & : 77 & & pasdutout & $=:$ & 14 \\
\hline Conditionnelpresent & $: 58$ & & rien & : & 12 \\
\hline Subjonctifpresent & $: \quad 43$ & & memepas & : & 9 \\
\hline (Other) & : 144 & & (Other) & : & 46 \\
\hline
\end{tabular}

Les tableaux en (177) nous dévoilent les occurrences des variantes de chaque variable et permettent ainsi une première impression de leur répartition globale dans le corpus. Étant donné qu'une classification binaire entre les sujets lexicaux et pronominaux n'est pas suffisante (cf. sections 2.2 .5 .1 et 2.2.5.2), nous avons aussi transformé la variable du type de sujet en une échelle numérique basée sur le nombre de segments du sujet ( $c f$. la préparation des données pour l'analyse multifactorielle, décrite dans la section 4.5.1 ci-dessus).

Ainsi, nous concevons non seulement que le nombre de pronoms en position de sujet est beaucoup plus élevé que celui des éléments lexicaux, mais également que les longueurs médiane ${ }^{134}$ (angl. median) et moyenne ${ }^{135}$ (angl. mean) des sujets dans le corpus sont d'environ deux segments, ce qui correspond, par exemple, à la longueur d'un clitique (cf. je [3ə]) ou d'un pronom relatif ( $c f$. qui [ki]). Nous observons par la suite qu'il y a deux fois plus de phrases principales que de subordonnées et que l'indicatif présent est le temps verbal le plus largement préféré dans le corpus.

La situation de communication et la provenance géographique sont réparties de manière à peu près équilibrée, comme le prévoit la conception du corpus ( $c f$. chapitre 3 ). Comme le type de sujet, le type de situation a, lui aussi, été transformé en une classification scalaire. Ceci nous permet une distinction plus fine entre les différents types de communication: les conversations reçoivent la valeur 1, les discussions en classe

134 L'élément médian d'une liste de valeurs est la valeur qui se trouve au milieu de la liste.

135 La moyenne est la mesure arithmétique de la valeur moyenne d'un groupe de valeurs. 
la valeur 2 et les examens la valeur 3 ( $c f$. la préparation des données pour l'analyse multifactorielle décrite dans la section 4.5 .1 ci-dessus). Finalement, l'emploi des éléments négatifs est le même dans l'ensemble du corpus que dans le sous-ensemble des énoncés négatifs, car seuls ces derniers comportent des éléments négatifs. En effet, comme nous l'avons déjà vu dans la section 4.4.3, pas est l'élément négatif largement préféré.

Après cette vue d'ensemble, nous entrons dans l'analyse détaillée des relations entre les variables directement ou indirectement pertinentes pour $\pm n e$. En (178) figure une vue d'ensemble des résultats.

(178) Les corrélations entre les variables dites 'indépendantes' dans Tzéro

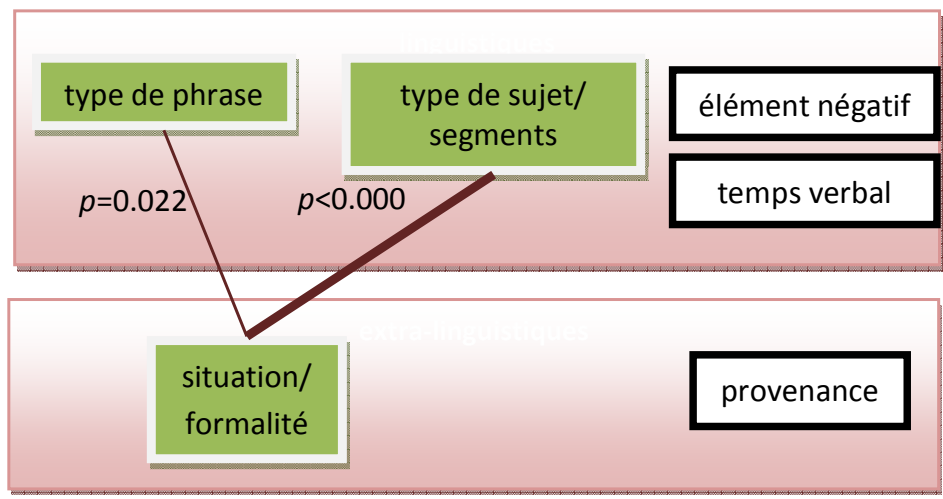

Dans le schéma (178), les relations entre les variables vertes qui sont pertinentes dans l'ensemble du corpus figurent encore sous forme de lignes. Effectivement, parmi toutes les combinaisons logiquement possibles des cinq variables dites 'indépendantes' en (178), il y a trois variables qui sont fortement dépendantes l'une de l'autre. Plus précisément, comme dans le sous-ensemble des énoncés négatifs, la situation de communication est en interaction avec les influences primaires sur $\pm n e$ dans T-zéro: d'un côté, elle interagit avec le type de sujet et de l'autre avec le type de phrase. Le facteur type de situation est donc en corrélation significative avec les types de sujet et de phrase, et ce non seulement par rapport à la variable $\pm n e$, mais aussi de façon générale 
dans le corpus. Cette tendance se manifeste d'ailleurs probablement dans tous les corpus contenant des données du langage spontané ( $c f$. la discussion en section 5.3).

Par contre, les influences secondaires sur \pm ne, à savoir le temps verbal, l'élément négatif et la provenance des locuteurs, $c f$. (178), semblent être indépendantes lesunes des autres, vu qu'elles ne manifestent aucune corrélation significative dans T-zéro. Ces corrélations se sont donc manifestées ponctuellement, seulement par rapport à la variable $\pm n e$ et seulement dans notre corpus et ne seront donc pas prises en considération dans la discussion qui suit.

Considérons maintenant la nature de l'interaction entre le type de situation et les deux facteurs linguistiques qui sont en interdépendance avec celui-ci, afin d'établir quel est le facteur qui exerce une influence sur l'autre et de quel sorte d'influence il s'agit.

\subsubsection{L'influence de la situation de communication sur l'emploi du type de sujet}

L'analyse portant sur la longueur de l'ensemble des sujets dans le corpus en fonction du degré de formalité de la situation en $\mathrm{R}$ a montré qu'il y a effectivement une corrélation significative $(p<0.000)$ entre ces deux variables: la longueur du sujet et la situation de communication.

La figure en (179) montre la relation entre le nombre de segments du sujet et le degré de formalité de la situation de communication. Nous distinguons entre les conversations libres 1 , les discussions en classe 2 et les examens oraux 3 ( $c f$. la préparation des données pour l'analyse multifactorielle, décrite dans la section 4.5.1 ci-dessus). 
(179) La longueur du sujet en segments par degré de formalité (immédiat communicatif: 1 , formalité moyenne: 2 , distance communicative: 3 )

Régression de la longueur du sujet par degré de formalité

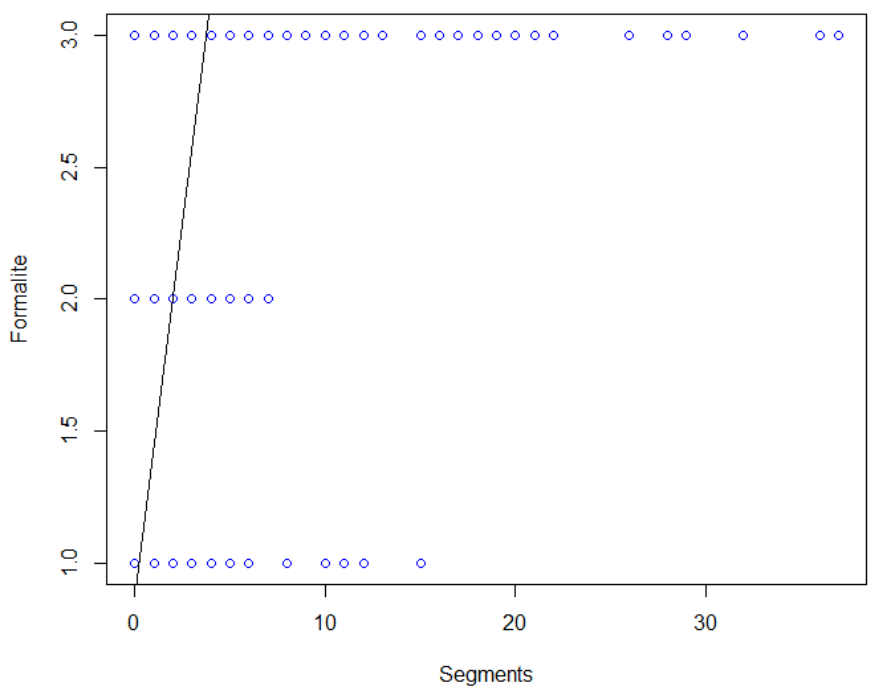

La longueur du sujet varie visiblement le plus dans les situations d'examen. Dans ce contexte, nous trouvons aussi bien des sujets zéro ou monosegmentaux que des sujets très longs, qui comptent jusqu'à plus de 30 segments, comme le montrent les exemples en (180).

(180) Exemples de sujets très longs (> 30 segments)

a. que [l'Albanie la Roumanie la France l'Angleterre et l'Allemagne] ont suivi la même évolution (2145)

b. [le renseignement sur le déroulement de ses journées] se manifeste (0180)

Dans les situations d'immédiat, par contre, la longueur des sujets varie moins : les sujets comptent entre 0 et 15 segments au maximum. 
(181) La longueur des sujets en fonction de la situation de communication

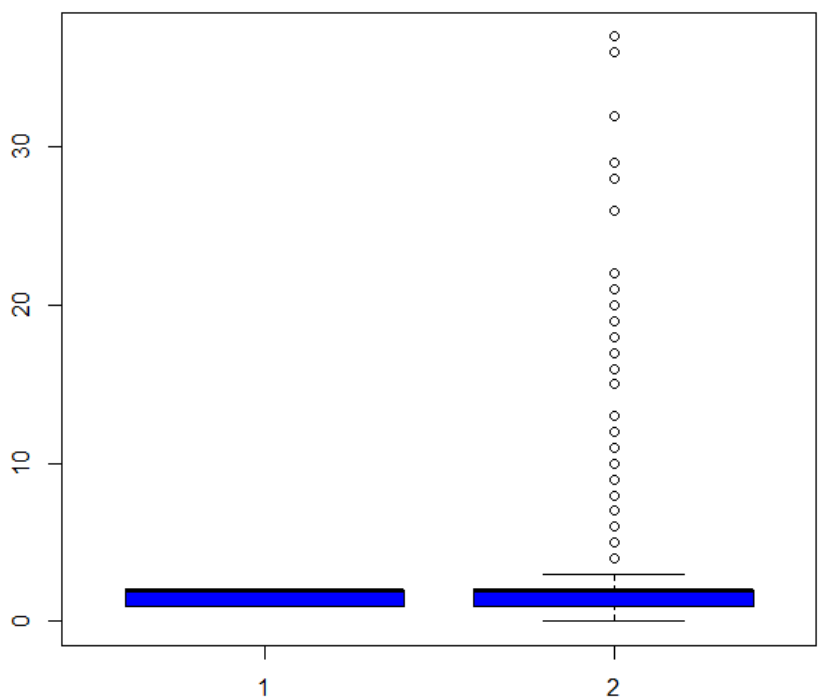

La comparaison directe des situations de l'immédiat (conversations et discussions en classe) avec la distance communicative (examens oraux) en (181) montre que la longueur moyenne du sujet (qui est environ de 2 segments, $c f$. (177)a dans la section 4.5.2 ci-dessus) est comparable dans les deux sous-corpus. Néanmoins, nous observons clairement que la variation de la longueur des sujets est beaucoup plus grande dans la distance communicative que dans l'immédiat.

Cela revient à dire Plus précisément, cela revient à dire que dans l'ensemble du corpus, mais en particulier dans l'immédiat communicatif, la fréquence des sujets légers et lourds est proportionnellement inverse à leurs taux de $\pm n e$, comme le diagramme détaillé en (182) le montre. 
(182) La fréquence des types de sujet selon la situation de communication

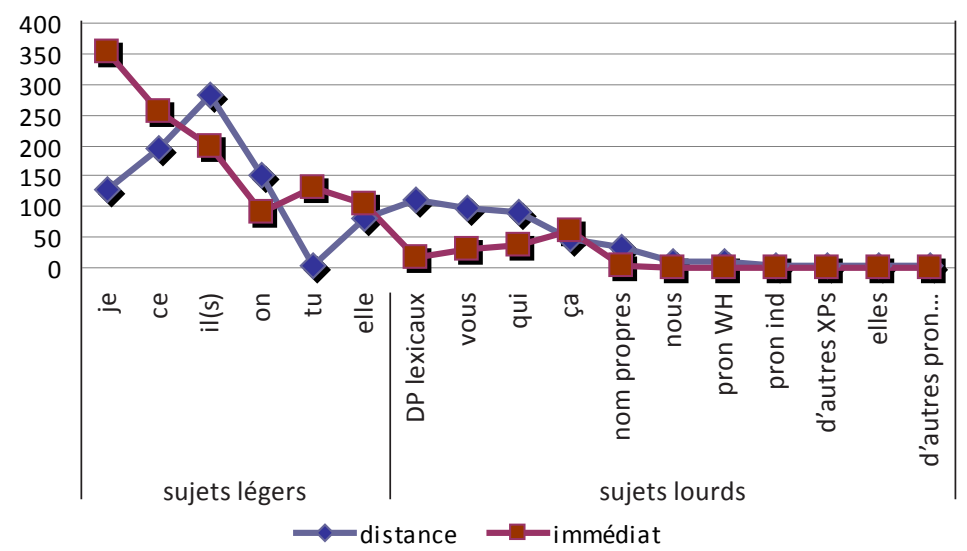

Dans l'immédiat communicatif, les sujets lourds sont extrêmement rares et manifestent des taux de ne très élevés, et, à l'inverse, les sujets légers sont très fréquents et manifestent des taux extrêmement restreints de $\pm n e$.

Par contre, la distance communicative montre une baisse frappante des types de sujet qui incitent à l'omission de $n e$ : le clitique léger je y est employé très rarement et le clitique tu est presque complètement absent. La distance communicative se caractérise par un taux élevé de $i l(s)$ (dont environ un quart sont impersonnels) et des DP lexicaux en position de sujet, qui incitent fortement à la réalisation de ne ( $c f$. section 2.2.5.1).

Nous pouvons donc retenir que, dans l'ensemble du corpus, la distribution des types de sujet dépend de manière significative de la situation de communication. Cette tendance n'est ni une observation nouvelle, ni une idiosyncrasie de notre corpus et a été observée pour la première fois dans une langue maya par Du Bois (1987), pour le français par Lambrecht (1987) et par la suite dans les corpus de nombreuses autres langues ( $c f$. les références citées en section 5.3). Cependant, les implications de cette tendance pour le traitement théorique de la variable $\pm n e$ n'ont jamais été exploitées en profondeur et seront donc discutées de manière détaillée dans la section 5.3. 


\subsubsection{L'influence de la situation de communication sur le type de phrase}

Il existe également une corrélation significative entre le degré de formalité et le type de phrase $(p=0,022)$.

(183) Le type de phrase en fonction de la situation de communication dans T-zéro (gris clair: subordonnée, gris sombre: principale, noir: non-identifiable)

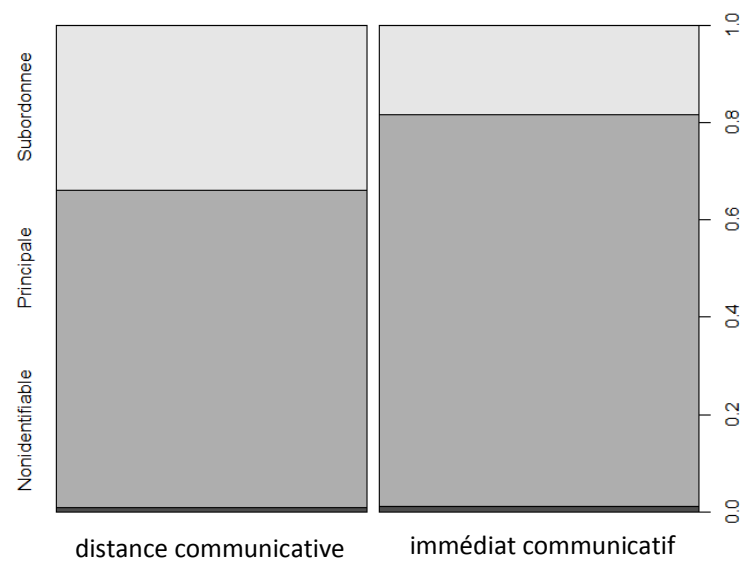

Nous observons en (183) que les phrases principales et les subordonnées sont distribuées de manière déséquilibrée à travers les situations de communication. Tandis que les principales (gris foncé) sont dominantes dans les deux situations de communication, la partie des subordonnées (gris clair) est plus grande dans le domaine de la distance communicative. Les deux situations manifestent, de plus, une ligne menue noire qui désigne les constructions non-identifiables.

Nous pouvons donc retenir que, comme la distribution des types de sujet, celle des types de phrase diffère également en fonction du degré de formalité de la situation de communication, ce qui n'est pas non plus une observation nouvelle. Koch/Oesterreicher (2011: 12) comptent la présence élevée des parataxes parmi les caractéristiques universelles du langage de l'immédiat communicatif, et Finegan/Biber (2001: 252) listent des études de corpus qui, comme la nôtre, classent l'emploi élevé 
des hypotaxes en tant que caractéristique typique de la distance communicative. La question de la signification de cette dépendance unilatérale entre la situation de communication et le type de phrase pour la variable $\pm n e$, qui, à notre connaissance, n’a pas encore été thématisée auparavant, sera discutée dans la section 5.3.

\subsubsection{Conclusion intermédiaire}

L'analyse multifactorielle nous a permis de détecter un réseau de facteurs dans lequel seuls les types de sujet, de phrase et de situation sont directement liés à la variable $\pm n e$. À l'aide de l'analyse des interactions générales entre les variables dites 'indépendantes', nous avons réussi, par la suite, à transformer le réseau de facteurs qui influencent $\pm n e$ (détecté lors de l'analyse multifactorielle dans la section 4.5.1) en une structure hiérarchique:

(184) La hiérarchie des facteurs qui influencent $\pm n e$

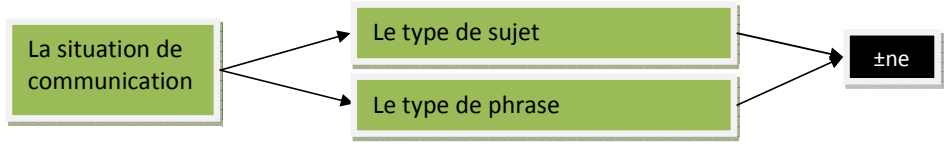

La situation de communication exerce une influence sur la distribution des types de sujet et de phrase, et ces deux derniers déterminent la distribution de la variable $\pm n e$ dans le discours: tandis que les sujets légers et les phrases principales, qui favorisent l'omission de ne, sont préférés dans l'immédiat communicatif, les sujets lourds et les phrases subordonnées, qui déclenchent la réalisation de ne, sont davantage représentés dans la distance communicative. La hiérarchie représentée en (184) et ses implications pour la théorie variationnelle et sociolinguistique par rapport à la variable $\pm n e$ seront discutées en détail dans la section 5.3. 


\subsection{Résumé du chapitre 4}

L'analyse globale dans le sous-chapitre 4.1 a montré que plus de $80 \%$ des 334 négations produites par les 54 locuteurs suisses et français dans le corpus sont monopartites.

Lors de l'analyse exploratrice dans le sous-chapitre 4.2, nous avons distingué trois types de locuteurs: le type A, qui manifeste une variation $\pm n e$, le type $\mathrm{B}$, qui montre l'absence de ne, et le type $\mathrm{C}$, qui montre une présence générale de ne. Le type A représente le francophone européen typique, il produit la majorité des négations dans le corpus, n'est pas déterminable par sa provenance géographique, son sexe, son âge ou sa profession et manifeste une la variation \pm ne dans différentes situations de communication. La raison principale pour laquelle nous n'observons aucune variation $\pm n e$ chez les locuteurs du type $\mathrm{B}$ et $\mathrm{C}$ est le nombre restreint de négations disponibles dans le corpus. À l'intérieur du type B, il y a certaines locutrices (uniquement de Suisse) qui semblent manifester l'absence de ne indépendamment du contexte morphosyntaxique et situationnel, mais seul un test d'acceptabilité permettrait de déterminer si l'assomption d'un type B également au niveau de la compétence est justifiée. Par contre, il n'y a aucune raison d'assumer que les locuteurs du type $\mathrm{C}$ aient fixé la réalisation constante de ne dans leurs grammaires. L'analyse a montré que, dans les cas en question, ce sont les caractéristiques linguistiques et situationnelles qui permettent d'expliquer l'emploi de ne.

Les analyses descriptives dans les sous-chapitres 4.3 et 4.4 se penchent en détail sur les facteurs (extra)linguistiques.

Au niveau intrapersonnel, qui est au cœur de la conception de notre corpus, l'analyse descriptive des facteurs extralinguistiques ( $c f$. souschapitre 4.3) fait surgir certains résultats significatifs et en partie inobservés jusqu'à présent. Premièrement, la comparaison des taux de ne dans les situations d'examen par rapport aux situations de conversation produit une différence statistiquement signifiante: dans les conversations, la réalisation de ne est nettement plus basse que lors des examens. Une telle distribution de la variable $\pm n e$ a déjà été proposée 
intuitivement, par exemple par Koch/Oesterreicher ( $\left.{ }^{2} 2011\right)$, mais sans appui empirique.

Deuxièmement, les différences de traitement de la variable $\pm n e$ entre les locuteurs suisses et français dans la distance communicative constitue un autre résultat important. Tandis que, lors des conversations, les taux de ne des Suisses et des Français sont pareils, il y a une différence significative dans leur comportement pendant les examens. Les Suisses omettent dans n'importe quelle situation la majorité des $n e$, alors que les Français corrigent leur production langagière de façon significative vers la norme dans une situation de distance. De plus, au niveau interpersonnel, la première exploration de l'influence du statut L1, bi- ou monolingue, et L2 dans l'analyse descriptive révèle une tendance intéressante: la réalisation du ne chez les locuteurs bilingues semble significativement inférieure à celle des locuteurs restants.

Par ailleurs, l'analyse reflète certaines tendances sociolinguistiques qui ne sont pas significatives dans nos données: la réalisation de ne semble moins fréquente en Suisse romande qu'en région parisienne, et les locuteurs plus âgés et possédant une formation académique supérieure tendent à réaliser plus de négations bipartites (surtout s'ils ont un rôle éducatif) que les élèves et les étudiants.

Au niveau linguistique ( $c f$. sous-chapitre 4.4), par contre, l'analyse descriptive détecte une série de facteurs statistiquement significatifs. Tout d'abord, une nouvelle classification morphophonologique des types de sujet est établie. À l'aide de celle-ci, des différences significatives ressortent entre les sujets légers, lourds et redoublés. Nous avons découvert qu'il y a, jusqu'à une longueur de deux syllabes, une relation stable entre la longueur d'un sujet simple et la réalisation de ne: tandis que les taux de $+n e$ avec des sujets lourds (par exemple les clitiques nous et vous, les pronoms indéfinis et les DP lexicaux) dépassent clairement la moyenne du corpus, pour ceux comportant des sujets légers (par exemple les clitiques de fréquence élevé comme je, tu, ce etc.) la réalisation de ne est très basse, voire inexistante. De plus, parmi les clitiques, il y a plusieurs variantes ( $c f$. [3ə], [3] et [ $\left.\int\right]$ pour je) et la particule de négation s'associe exclusivement avec la forme standard (donc avec [3ə]), tandis que les autres variantes ne sont jamais réalisées avec ne. 
Le dernier point important par rapport au facteur du sujet est l'absence totale de ne avec les sujets redoublés, que nous observons dans notre corpus. Cette absence semble un épiphénomène de la forte présence de pronoms sujets légers et en particulier de leurs variantes monosegmentales incompatibles avec ne. De plus, les réalisations de ne dans une séquence proclitique semblent des hypercorrectismes, produits uniquement pendant les examens.

$\mathrm{Au}$ niveau de la construction syntaxique de la phrase, la réalisation de ne est significativement plus fréquente dans les subordonnées que dans les principales. Il y a même une différence (non-significative) entre les relatives, qui montrent un taux de ne supérieur, et les autres types de subordonnées. De même, au niveau phonétique, il y a des influences significatives sur la variable $\pm n e$. Les contextes V_V et \#_C incitent à la réalisation de $n e$, tandis que la tendance générale à l'omission $\mathrm{du}$ ne se manifeste dans les contextes $\mathrm{V} \_\mathrm{C}, \mathrm{C} \_\mathrm{C}$ et $\mathrm{C} \_\mathrm{V}$. Nous notons que les syllabes pleines semblent 'protéger' ne: dans les contextes qui le favorisent, $n(e)$ est toujours réalisé comme une syllabe CV.

En ce qui concerne les facteurs linguistiques restants, nous ne constatons aucune influence indépendante sur $\pm n e$, mais plutôt des effets de fréquence. Ceci signifie que les formes très fréquentes manifestent des valeurs de $+n e$ (parfois significativement) inférieures par rapport aux formes restantes, sans avoir pourtant un impact linguistique sur $\pm n e$ (contrairement aux facteurs du type de sujet et de phrase). Parmi les éléments négatifs non-clitiques, l'omission de ne est plus forte avec l'élément fréquent pas qu'avec d'autres éléments (surtout si ceuxci, comme personne ou rien, sont employés en tant que sujets lourds).

Quant à l'influence du verbe, nous constatons que les verbes peu fréquentsincitent tendanciellement à la réalisation de $n e$, tandis que les verbes très fréquents favorisent l'omission de ne. Ce sont les verbes d'action et lexicaux qui provoquent les taux de ne les plus élevés. Cependant, les verbes de procès et avoir/être se situent au milieu et les verbes d'état et modaux manifestent les taux de ne les plus restreints. Tandis que les valeurs du présent et du passé composé reflètent à peu près la moyenne du corpus, l'imparfait et le futur périphrastique manifestent des taux de + ne nettement plus bas. Dans ce contexte, nous avons pu 
réfuter une 'règle' formulée par Ashby (1981: 680), qui soutient que la réalisation de $n e$ avec les impératifs était catégorique.

Finalement, dans le sous-chapitre 4.5 , nous avons réussi, à l'aide d'une analyse multifactorielle, à séparer les influences significatives et directes sur $\pm n e$ des influences indirectes et à représenter ainsi les influences sur \pm ne sous forme d'un réseau complexe. L'analyse multifactorielle confirme les tendances qui se sont montrées déjà en partie lors de l'analyse descriptive, à savoir que le type de sujet et le type de phrase ainsi que la situation de communication exercent directement des influences significatives sur $\pm n e$. Par contre, l'élément négatif, le temps verbal et la provenance des locuteurs sont en corrélation avec la situation de communication et n'influencent, à travers celle-ci, la variable \pm ne qu'indirectement.

Par la suite, une analyse détaillée des interactions entre les variables qui influencent \pm ne directement ou indirectement, effectuée sur l'ensemble du corpus, a permis de structurer le réseau des facteurs qui influencent \pm ne et d'identifier une hiérarchie entre ceux-ci. Nous avons vu que les interactions entre les types de sujet et de phrase, d'un côté, et la situation de communication, de l'autre, représentent des tendances générales de notre corpus, qui sont indépendantes de la variable $\pm n e$. Nous avons pu montrer, par la suite, que c'est la situation de communication qui exerce une influence considérable sur la distribution des types de sujet et de phrase: dans l'immédiat communicatif, les sujets légers et les parataxes (qui incitent à l'omission de ne) sont largement préférés, tandis que, dans le distance communicative, le pourcentage des sujets lourds et des hypotaxes (qui favorisent la réalisation de ne) est élevé.

Les implications théoriques de cette observation empirique seront discutées en détail dans la section 5.3. 
Charlotte Meisner - 978-3-0343-2332-1

Downloaded from PubFactory at 01/11/2019 02:38:59AM

via free access 


\section{Discussion des résultats}

\section{1 Évaluation des approches de la variation $\pm n e$ face aux résultats de l'analyse}

\subsubsection{L'approche externe: \pm ne comme variable sociolinguistique}

La variable \pm ne est souvent décrite comme une variable sociolinguistique (cf. Armstrong/Smith 2002: 23, Coveney ${ }^{2} 2002: 55$, Gadet 1997b, Labov 1972a,b) qui est en covariation relativement stable avec des facteurs extralinguistiques. Les facteurs sociolinguistiques 'classiques' que nous avons testés sont l'âge, la profession et le niveau de formation.

Avant de passer à l'évaluation de l'hypothèse du statut de $\pm n e$ en tant que variable sociolinguistique, nous tenons à noter qu'à cause de sa taille restreinte et sa focalisation sur la variation intrapersonnelle ( $c f$. sections 3.1, 3.2 et 3.3), notre corpus n'est pas idéalement conçu pour tester cette hypothèse. Ceci concerne aussi le fait que les différents facteurs sociodémographiques coïncident parfois dans certains de nos locuteurs: les individus plus âgés sont ceux qui possèdent une formation élevée et qui exercent une profession dans le domaine de l'éducation. De plus, les données de certains professeurs proviennent uniquement des examens oraux et ne permettent donc aucune évaluation intégrale de leur comportement verbal.

Malgré ces quelques limitations du corpus, l'analyse descriptive a permis la confirmation de certaines tendances sociolinguistiques générales (même si celles-ci ne sont pas statistiquement significatives). En effet, tout comme le soutiennent Ashby (2001), Armstrong/Smith (2002) et Blanche-Benveniste/Jeanjean (1987), les locuteurs plus âgés produisent des taux de $+n e$ plus élevés que les jeunes, et les professions dans le domaine de l'éducation, qui demandent une formation supé- 
rieure, incitent ultérieurement à la réalisation de $n e$ (cf. Ashby 1976, Coveney ${ }^{2} 2002$ et Hansen/Malderez 2004).

Toutefois, mis à part la langue maternelle, aucun des facteurs interpersonnels ne produit un effet statistiquement significatif sur la réalisation de $n e$. De plus, même les analyses sociolinguistiques à grande échelle (cf. Ashby 1976, 1981, 2001, Coveney ${ }^{2} 2002$ ) donnent lieu à des résultats contradictoires quant à l'hypothèse du \pm ne comme variable sociolinguistique: dans l'étude d'Ashby (1976), par exemple, la couche 'populaire' manifeste les taux de $+n e$ les plus élevés, tandis qu'elle montre les taux les plus bas dans Ashby (2001) (cf. section 2.2.4.3).

D'une manière générale, les analyses sociolinguistiques ne permettent pas de reconnaître si la variable $\pm n e$ devrait être traitée comme un indicateur, un marqueur ou un stéréotype variationnel. Autrement dit, la question de savoir s'il s'agit d'une variable qui indique une stratification sociale mais pas stylistique, une stratification aussi bien sociale que stylistique, ou si elle a gagné une attention consciente parmi les locuteurs et ne fonctionne donc plus comme variable sociolinguistique discrète reste ouverte.

Il reste donc difficile, selon nous, de traiter $\pm n e$ comme une variable sociolinguistique. Ceci devient encore plus clair si l'on considère que les facteurs linguistiques, qui sont extrêmement importants pour la variation de $\pm n e$, comme il a été montré non seulement lors de notre analyse, mais également par de nombreux autres chercheurs (cf. entre autres Ashby 1976, 1981, 2001, Armstrong/Smith 2002, Hansen/Malderez 2004), ne sont aucunement intégrés dans l'hypothèse de $\pm n e$ comme variable sociolinguistique. Or, il faudrait expliquer pourquoi un locuteur de classe sociale élevée devrait employer davantage de sujets lourds (qui incitent à la réalisation de $n e, c f$. section 4.4.1.1) qu'un locuteur de classe sociale moyenne ou basse. Dans l'ensemble, les locuteurs du type A, qui manifestent la variation de $\pm n e$, ne sont pas déterminables par leurs caractéristiques sociodémographiques. En d'autres termes, dans les zones francophones représentées dans notre corpus, la variation $\pm n e$ est un phénomène largement répandu à travers toute la société et non pas une caractéristique d'un certain groupe sociodémographique. 


\subsubsection{L'approche interne: l'hypothèse de l'incompatibilité entre ne et d'autres clitiques}

L'idée d'une exclusion mutuelle de ne et d'autres clitiques (cf. Ashby 1977, Culbertson/Legendre 2008, Larrivée 2014, Posner 1985: 189) semble, à priori, bien refléter certains faits observés dans notre corpus. Comme illustré schématiquement dans le tableau (3) (répété sous (185)), les données généralement trouvées dans les corpus de français spontané (aussi dans le nôtre) confirment une certaine tendance à l'incompatibilité entre les clitiques légers et $n e$.

(185) \pm ne selon le type de sujet: le patron de variation souvent observé dans les corpus

\begin{tabular}{|l|l|l|}
\hline Sujet & \multicolumn{1}{l|}{ $\pm n e$} & exemple du corpus \\
\hline -clitique & $+n e$ & a. Ulisse ne montre aucun intérêt dans le dialogue (0046) \\
\hline +clitique & $-n e$ & b. $\boldsymbol{j}$ 'ai pas grand chose à dire (0134) \\
\hline
\end{tabular}

Dans notre corpus, le type de sujet grammatical possède une influence solide sur la variable $\pm n e$ ( $c f$. section 4.4.1). Tandis que les sujets phonologiquement lourds (sujets lexicaux, noms propres, pronoms indéfinis négatifs) incitent à la réalisation de ne, $c f$. (82)a, les sujets phonologiquement légers, comme par exemple les pronoms clitiques je, $t u, i l$ ou $c e$ (qui sont en même temps les plus fréquents, $c f$. section 5.3), semblent empêcher la réalisation de $n e, c f$. (82)b.

Toutefois, l'hypothèse de l'incompatibilité entre ne et d'autres clitiques pose une série de problèmes par rapport aux données empiriques. Tout d'abord, la tendance observée n'est pas sans exception, comme en témoignent les contre-exemples (très rares mais existants) tirés de Tzéro en (186):

(186) Contre-exemples au patron 'typique' de la variation $\pm n e$

\begin{tabular}{|lll|}
\hline Sujet & $\pm n e$ & exemple du corpus \\
\hline -clitique & $-n e$ & a. la personne suivante est pas là (0869) \\
+clitique & $+n e$ & b. je n'ai pas envie (0108) \\
\hline
\end{tabular}


Ensuite, non pas tous les clitiques provoquent l'effacement de ne: les analyses de corpus ont montré qu'il y a des différences considérables quant à la présence de ne à l'intérieur du paradigme des clitiques. Par exemple, nous et vous manifestent des taux de ne beaucoup plus élevés que je et il ( $c f$. sections 2.5.1 et 4.4.1). Cette tendance reste donc relativement difficile à modeler, du moins en termes d'une règle syntaxique (pour une proposition dans le cadre de la morphologie distribuée, $c f$. Meisner/Pomino 2014).

Dans l'ensemble, une règle syntaxique qui exclut ne en présence d'autres proclitiques semble trop restrictive pour répondre à la réalité linguistique. Cependant, même si une incompatibilité généralisée (cf. Ashby 1977, Culbertson/Legendre 2008, Larrivée 2014, Posner 1985: 189) entre ne et d'autres clitiques est improbable, il y a de fortes évidences en faveur d'une incompatibilité entre ne et certaines variantes clitiques. Ceci est particulièrement saillant dans les séquences proclitiques où l'omission de ne semble beaucoup plus naturelle que sa réalisation. Les propriétés phonotactiques des séquences en question seront discutées dans la section 5.2.4.2.

\subsubsection{L'approche diglossique: l'hypothèse des deux grammaires du français}

Culbertson (2010), Culbertson/Legendre (2008), Massot (2010), Mensching (2008), Koch/Oesterreicher (2011) et Zribi-Hertz (2011) proposent d'analyser la variation $\pm n e$ en termes d'une alternance codique entre une variété de l'immédiat (grammaire 1), sans ne, acquise de manière naturelle de génération en génération, et une variété de distance (grammaire 2), qui est acquise à l'école et qui comprend la particule ne. Selon Culbertson (2010), la grammaire 1, qui génère uniquement la négation monopartite, est une langue à sujet nul contenant des marques personnelles affixées au lieu des proclitiques, tandis que dans la grammaire 2, qui comprend une négation bipartite, les clitiques sont analysés comme des arguments pleins, parallèlement aux DP lexicaux (cf. section 2.3.3). Cependant, mis à part l'analyse de corpus de Massot 
(2010), portant sur les données linguistiques d'un seul locuteur, cette approche n'a jamais été testée empiriquement.

L'approche diglossique selon Culbertson (2010), Mensching (2008), Koch/Oesterreicher (2011) et Zribi-Hertz (2011) fait des prédictions claires concernant la distribution de nombreuses variantes linguistiques, dont certaines peuvent être testées dans notre corpus.

Cette approche implique d'abord que l'absence de ne est plus articulée dans les situations de l'immédiat que dans la distance communicative, puisque la grammaire 1 y serait davantage utilisée. Deuxièmement, l'absence de ne devrait être en cooccurrence dans les corpus avec les autres variantes attribuées à la grammaire 1, tandis que la présence de $n e$ serait en cooccurrence stable avec les variantes attribuées à la grammaire 2. Troisièmement, en se basant sur Morin (1979) et Fonseca-Greber/Waugh (2002), Culbertson (2010: 91) soutient que la diglossie francophone se manifeste au niveau des variantes des clitiques sujet: les formes réduites, comme $\left[\int\right],[\mathrm{t}]$ ou [i], appartiendraient à la grammaire 1, tandis que les variantes standard, comme [3ə], [ty] et [i1], appartiendraient à la grammaire 2. Selon l'approche diglossique, nous nous attendrions donc à ce que, comme la variable $\pm n e$, les variantes des clitiques sujet se distribuent, elles aussi, selon les situations de communication: les variantes 'affixées' de la grammaire 1 seraient davantage utilisées dans les situations de l'immédiat communicatif, tandis que les 'vrais clitiques' de la grammaire 2 seraient réservés aux situations de distance communicative.

Dans notre corpus, la première condition est à peu près remplie: dans les situations de l'immédiat, les locuteurs montrent une moyenne de $5 \%+n e$, tandis que dans les examens oraux, la réalisation de $n e$ atteint $35 \%+n e$ ( $c f$. section 2.4.4.4). Selon la théorie diglossique, ce premier résultat pourrait signifier que les locuteurs alternent continuellement entre les deux variétés, mais qu'ils préfèrent généralement la grammaire 1 (sans ne), puisque l'absence de ne est représentée aussi bien dans les situations de l'immédiat que dans celles de distance communicative. Ceci revient à dire que les locuteurs utiliseraient aussi la grammaire 1 dans les situations de distance, vu qu'avec 35\% +ne l'absence de la variable y est toujours plus fréquente que sa présence. La 
seule différence entre les deux situations résiderait donc dans le fait que, dans la distance communicative, les locuteurs emploient de temps en temps des énoncés produits dans la variété standard (grammaire 2), ce qui n'est presque jamais le cas dans les conversations.

Or, étant donné que dans n'importe quelle situation, la majorité des négations sont monopartites, il est nécessaire d'expliquer pourquoi la variété standard est utilisée de manière généralement faible. S'agit-il d'un processus social de 'démocratisation' dans le domaine de la distance communicative phonique ( $c f$. le concept de social shift selon Posner 22007: 57-101), qui fait que la variété de l'immédiat y devient peu à peu acceptable? Ou bien, ces résultats montrent-ils tout simplement qu'il n'y a pas de diglossie dans le monde francophone et que la variation observée relève d'une même grammaire? Dans ce qui suit, nous allons présenter quelques arguments en faveur de cette dernière position.

Considérons maintenant le deuxième critère déterminant pour l'hypothèse diglossique, à savoir la covariation présupposée entre $\pm n e$ et les variantes attribuées respectivement aux deux grammaires. Massot (2010: 98) soutient que les variantes des deux grammaires ne sont jamais mélangées au niveau d'un même énoncé. Vu que T-zéro contient uniquement des constructions verbales ( $c f$. section 3.1), c'est-à-dire des énoncés minimaux, ce critère semble parfaitement testable dans notre corpus. L'évaluation du critère de la cooccurrence des variables au niveau de l'énoncé figure en tableau (187):

(187) La covariation entre \pm ne et les autres variantes attribuées aux grammaires 1 et 2

(cf. Massot 2010: 201, Koch/Oesterreicher 22011: 167-181 et Zribi-Hertz 2011: 6-7)

\begin{tabular}{|c|c|c|c|c|c|}
\hline & Grammaire 1 & $\%+n e$ & Grammaire 2 & \multicolumn{2}{|c|}{$\%+n e$} \\
\hline Interrogation & il vient? & $0 / 13$ & vient-il? & $1 / 1$ & (:) \\
\hline \multirow[t]{5}{*}{ Pronoms } & faut & $0 / 2$ & il faut & $0 / 2$ & 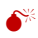 \\
\hline & [ja] & $0 / 3$ & il ya & $2 / 12$ & $\sigma^{\prime \prime}$ \\
\hline & {$[\mathrm{i}],[\mathrm{t}]$} & $5 \%$ & $i l, t y$ & $19 \%$ & $?$ \\
\hline & on & $9 \%$ & nous & - & r \\
\hline & $c ̧ a$ & $19 \%$ & cela & - & - \\
\hline Relative & que relatif sujet & - & qui relatif sujet & $8 / 15$ & ? \\
\hline
\end{tabular}




\begin{tabular}{|c|c|c|c|c|c|}
\hline \multirow[t]{2}{*}{ Accord } & $\begin{array}{l}\text { les lettres }{ }_{\mathrm{Pl.F}} \\
\text { que j'ai [ekbi] }\end{array}$ & - & $\begin{array}{l}\text { les lettres } \\
\text { que j'ai [eksit] Pl.F }\end{array}$ & $0 / 1$ & 要 \\
\hline & $c^{\prime} e^{2} t_{3 \mathrm{SG}}$ mes amis $3 \mathrm{Pl}$ & $0 / 5$ & ce sont ${ }_{3 \mathrm{Pl}}$ mes amis $3 \mathrm{Pl}$ & $1 / 1$ & (;) \\
\hline $\begin{array}{l}\text { Redoublement du } \\
\text { sujet }\end{array}$ & $\begin{array}{l}\text { moi je viens, Pierre } \\
\text { il vient }\end{array}$ & $0 / 34$ & je viens, Pierre vient & $18 \%$ & $?$ \\
\hline \multirow[t]{2}{*}{ Phrases clivées } & c'est Xqui, & $0 / 3$ & \multirow[t]{2}{*}{ SVO } & $19 \%$ & r \\
\hline & ce que/qui...c'est... & - & & & \\
\hline \multirow[t]{3}{*}{ Temps et mode verbal } & il a été & $17 \%$ & il fut & - & r \\
\hline & je vais aller & $0 / 9$ & j'irai & $1 / 1$ & (;) \\
\hline & je voulais qu'il vienne & - & je voulais qu'il vînt & - & \\
\hline Article indéfini pluriel & des bons vins & - & de bons vins & - & \\
\hline liaison 'facultative' & $\begin{array}{l}\text { c'est\# un esprit } \\
\text { important }\end{array}$ & $0 / 25$ & $\begin{array}{l}\text { il es }[\mathrm{t}] \text { un esprit } \\
\text { important }\end{array}$ & $2 / 6$ & $\sigma$ \\
\hline
\end{tabular}

Dans le tableau (187), nous présentons les variantes linguistiques qui, selon Massot (2010: 201), Koch/Oesterreicher (2011 : 167-181) et ZribiHertz (2011: 6-7), appartiennent à l'une des deux grammaires du français (cf. aussi Culbertson 2010). Il s'agit essentiellement d'une reprise du tableau (85) de la section 2.3.3, la seule différence étant que nous indiquons ici dans les deux colonnes intitulées $\%+n e$ la proportion respective de cooccurrences entre la variable $\pm n e$ et les autres variables en question qui sont correctement pronostiquées par l'approche diglossique. Les symboles dans la dernière colonne du tableau (187) montrent si le critère de la cooccurrence est atteint $(\odot)$, tendanciellement atteint (?) ou pas du tout atteint ( $\left({ }^{*}\right)$.

Ainsi, nous lisons dans la première ligne du tableau qu'il y a treize questions intonatives, $c f$. (188)a, qui sont attribuées à la grammaire 1 de l'immédiat et dont aucune n'est en cooccurrence avec $+n e$. En revanche, la seule question éventuellement inversée dans un contexte négatif contient la particule ne, $c f$. (188)b.

(188) Les interrogatives intonatives vs. inversées

a. on peut pas faire les bruits des animaux? (0656)

b. ce n'est(-il) pas la Dalmatie? (1399) $)^{136}$

136 Le fait que l'élément (-il) est mis entre parenthèses signifie qu'il n'est pas parfaitement audible dans l'enregistrement ( $c f$. le protocole de transcription pré- 
Nous rappelons que la grammaire 1 n'est censée générer que la négation monopartite, tandis que la grammaire 2 génère toujours les négations bipartites. Selon cette approche, nous nous attendons donc à une réalisation zéro de $n e$ avec les variantes de la grammaire 1 et à une réalisation stable avec les variantes de la grammaire 2. Concernant la formation des interrogatives, présentée dans la première ligne du tableau (187), la cooccurrence avec \pm ne est donc correctement prédite par l'approche diglossique, comme le désigne le symbole $\odot$.

Dans l'ensemble, seules trois variables montrent une covariation parfaite avec $\pm n e$ : la formation des interrogatives décrite ci-dessus, l'accord sujet-verbe ( $c f$. (189)) et la forme du futur ( $c f$. (190)). Mis à part l'objection que le nombre d'interrogations niées est très limité dans ce corpus, l'on pourrait soutenir que ne est toujours absent avec les interrogatives intonatives, c'est + pluriel et avec le futur périphrastique, tandis que sa réalisation est absolue avec l'inversion complexe, la tournure ce sont+pluriel et le futur simple.

(189) c'est + pluriel vs. accord correct

a. $\quad$ c'est pas mal les Autrichiens (0756)

b. des systèmes philosophiques qui ensuite ne sont pas applicables (1026)

(190) Le futur périphrastique vs. simple

a. moi je vais pas lire en allemand (0664)

b. alors qu'il avait dit qu'il n'aura pas de problèmes avec ses amis (1006)

Toutefois, la plupart des variables ne montre aucune covariation dans le sens de l'approche diglossique ou ne manifeste que des tendances qui ne sont pas formalisables en termes de deux grammaires.

Plus précisément, il est vrai que les formes [ja], faut, [i] et [t] ne sont jamais en cooccurrence avec ne, mais leur contreparties il y a, il faut, [il] et [ty] ne le sont pas non plus. Les pronoms nous et cela ne manifestent aucune occurrence dans une phrase négative dans le corpus, cependant la réalisation de ne avec leur variantes on et ça n'est

senté dans la section 3.7), mais vu qu'il s'agit du seul exemple potentiel dans ce cas, il est n'a pas été exclu de l'évaluation. 
clairement pas de zéro, comme le prédirait l'approche diglossique.Les relatives en qui montrent elles aussi un taux de ne relativement élevé, mais il n'atteint jamais $100 \%$ des cas ${ }^{137}$. Pour l'accord du participe passé, nous ne trouvons qu'un seul exemple négatif, $c f$. (191). Dans celui-ci, l'accord est exprimé, mais ne est omis, ce qui selon l'approche diglossique devrait être impossible.

(191) parce que vous dites des choses qui sont pas fausses (0136) [passkəvuditdefozkisว̃ pafos]

Nous avons déjà vu dans la section 4.4.1.3 qu'avec les sujets redoublés, la réalisation de ne est de zéro, comme le prédit l'approche diglossique:

(192) moi je vais pas lire en allemand (0664)

Ce fait, également observé par Massot (2010), amène celui-ci à conclure qu'il y a effectivement une diglossie en France. Toutefois, Massot (2010) ne teste pas l'implication inverse, à savoir, si la réalisation de ne avec les sujets simples est stable, comme la théorie de la diglossie le prédit. Dans notre corpus, le taux de $+n e$ avec les sujets non-redoublés ne correspond environ qu'à la moyenne du corpus, qui est de $18 \%+n e$, ce qui constitue une preuve contre la théorie des deux grammaires.

La même observation est valable pour les constructions clivées (cf. section 4.4.5.2): dans les trois clivées négatives trouvées dans le corpus, ne est absent (cf. (193)), mais par contre dans les énoncés suivant l'ordre des mots non-marqué SVO, sa réalisation est de $19 \%+n e$.

(193) c'est pas [NomPr] qui est en train de parler (1996)

L'emploi de $\pm n e$ avec le passé composé correspond lui aussi à la moyenne du corpus, tandis que le passé simple n'apparaît dans aucun énoncé négatif. Finalement, selon l'approche diglossique, +ne serait censé se regrouper avec les liaisons facultatives réalisées.

137 Les relatives négatives dans lesquelles que est employé comme sujet sont marginales et ne peuvent pas être vérifiées. 
(194) Liaison facultative

a. c'est pas nous qu'on veut \# être naïfs (0466)

b. ce n'est pas [z] une religieuse (1517)

c. que le glossaire de Reichenau n'a pas \# été une (option?) (1625)

En effet, comme prédit par l'hypothèse diglossique, +ne n'est jamais en cooccurrence avec une liaison supprimée, $c f$. (194)a. Si ne est réalisé, ceci accroît les possibilités d'une liaison, $c f$. (194)b. Cependant, il y a également des occurrences de + ne sans liaison, comme celle en (194)c.

Pour conclure sur le critère de la cooccurrence entre \pm ne et les autres variantes attribuées aux deux grammaires du français, nous constatons que la cooccurrence entre ces variantes est plutôt marginale et inconsistante. Jusqu'à ce point de la discussion, il n'y a aucun indice empirique de l'existence de deux variétés distinctes, entre lesquelles les locuteurs alternent, comme le soutiennent Culbertson (2010), Massot (2010), Zribi-Hertz (2011) et Koch/Oesterreicher (2011). Au contraire, il semble que les locuteurs mélangent librement les variantes attribuées aux deux grammaires. Pour maintenir l'hypothèse de la diglossie francophone, nous serions donc forcés d'admettre une vaste gamme de variation à l'intérieur des deux grammaires. Cependat, ceci n'est pas souhaitable, vu que l'hypothèse a été introduite dans le seul but d'expliquer la variation observée en français.

Le troisième critère pour l'évaluation de l'approche diglossique concerne les variantes des clitiques sujet qui devraient refléter (selon Culbertson 2010: 91, cf. aussi Culbertson/Legendre 2008) l'existence des deux grammaires. Ce critère a été testé par Meisner/Pomino (2014) sur notre corpus et nous allons résumer à continuation les résultats les plus importants de cette évaluation.

Meisner/Pomino (2014) constatent qu'il serait effectivement possible de projeter les variantes clitiques trouvées dans notre corpus ( $c f$. le tableau (62) dans la section 2.2.5.2) sur les deux grammaires proposées par les défenseurs de l'approche diglossique. Cette idée est illustrée dans la figure sous (195), où tous les éléments dans la section grise appartiendraient à la grammaire 1. Ces éléments sont monosegmentaux et seraient des préfixes flexionnels du verbe qui ne peuvent jamais être en cooccurrence avec ne ( $c f$. section 2.3.3). Par contre, les éléments dans 
les cases blanches seraient forcément ambigus, vu qu'ils sont parfois en cooccurrence avec ne et parfois non, c'est-à-dire qu'ils appartiendraient en même temps aux deux grammaires: dans la grammaire 1, ils seraient des préfixes incompatibles avec ne et dans la grammaire 2, de vrais clitiques permettant l'insertion de $n e$.

(195) Les variantes clitiques dans notre corpus selon l'hypothèse diglossique ${ }^{138}$ (cf. Meisner/Pomino 014)

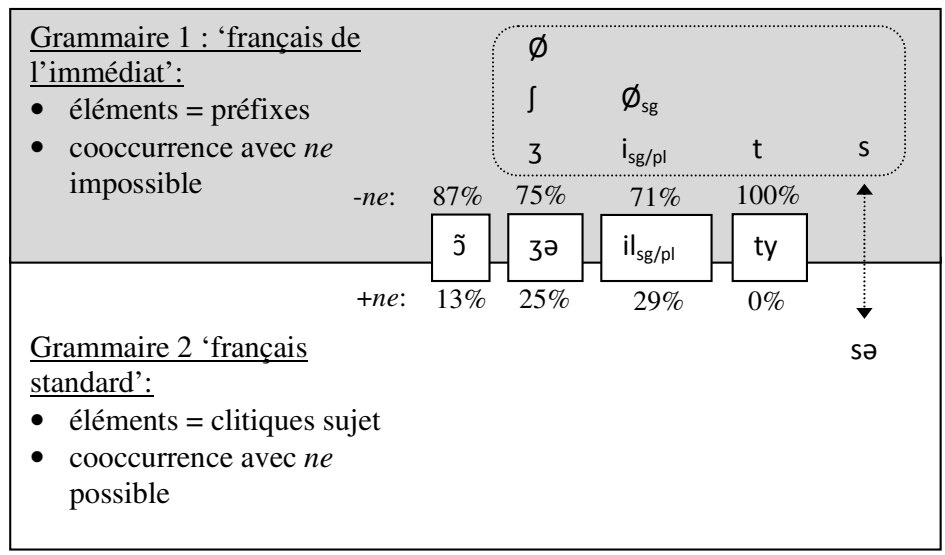

Meisner/Pomino (2014) concluent que la possibilité de 'distribuer' les variantes clitiques sur les deux grammaires comporte plusieurs désavantages. Tout d'abord, l'on se demande pourquoi il y a dans la grammaire 1 aussi bien des formes fortement érodées que des formes bisegmentales. Nous nous attendrions plutôt à une distribution binaire sur les deux grammaires, comme celle du démonstratif $c e$ : la forme monosegmentale [s] n'est jamais en cooccurrence avec ne et la forme bisegmentale [sə] manifeste une présence absolue de ne.

138 Notons que [3] existe aussi en français standard (= grammaire 2), mais uniquement s'il précède une voyelle. La variante [3] ne sera jamais attestée dans un contexte négatif en français standard, sauf si nous assumons aussi l'omission de ne dans cette variété (ex. j'arrive pas vs. je n'arrive pas). 
Ensuite, nous pouvons nous demander si la grammaire 2, donc le français standard, existe vraiment hors des manuels de grammaire, et si oui, quelles seraient ses propriétés: au niveau des pronoms, il n'y aurait qu'une seule forme, à savoir [sə], qui appartiendrait clairement et exclusivement à cette variété. Pour conclure sur les variantes pronominales, l'évaluation du critère des formes clitiques nous amène lui aussi à refuser l'hypothèse diglossique.

Le dernier critère pour l'évaluation de l'approche diglossique concerne la distribution des variantes clitiques dans notre corpus. Nous nous attendrions à ce que les variantes attribuées à la grammaire 1 soient davantage utilisées dans les situations de l'immédiat, tandis que celles pouvant appartenir à la grammaire 2 apparaissent plutôt dans les situations de distance communicative. Ce dernier critère a lui aussi été évalué par Meisner/Pomino (2014) et leurs résultats quant à la distribution des formes clitiques dans notre corpus figurent sous (196).

(196) Les variantes des clitiques sujet dans les sous-corpus de l'immédiat et de la distance communicative ${ }^{139}$ (cf. Meisner/Pomino 2014)

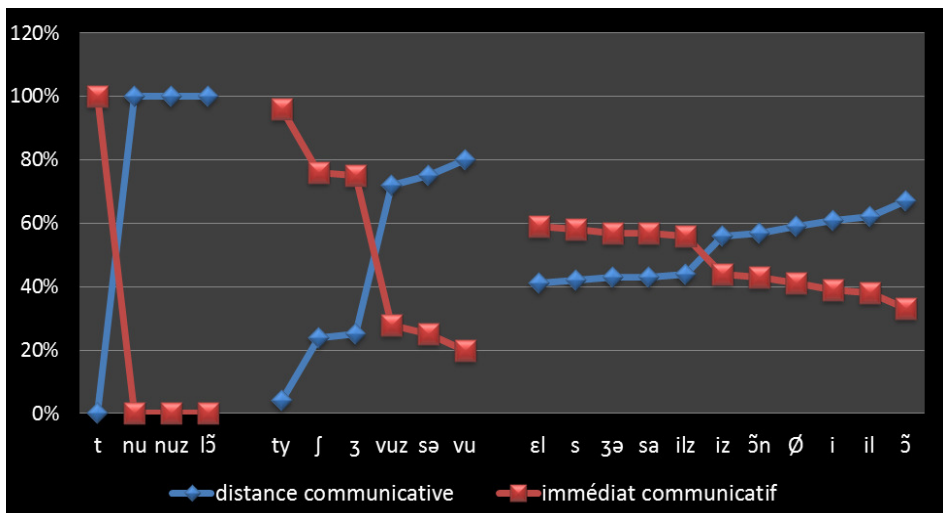

Le diagramme (196) montre que seul un nombre restreint de variantes semble appartenir clairement à l'un des deux sous-corpus : ceci vaut pour la variante [t] de $t u$, qui apparaît uniquement dans le corpus de l'immédiat

139 Seulement les formes avec plus d'une occurrence ont été considérées. 
(cf. la ligne rouge), tandis que [nu]/[nuz] et la variante impersonnelle l'on, qui est marquée comme extrêmement soutenue, sont restreintes au sous-corpus de la distance communicative ( $c f$. la ligne bleue).

Tous les autres éléments sont présents dans les deux sous-corpus et se distinguent uniquement par leurs fréquences. Dans le corpus de la distance, les occurrences de $t u$ et je sont rares, tandis que nous et vous apparaissent plus fréquemment. Les variantes de la troisième personne et de on ainsi que [3ə] sont représentées de manière assez équilibrée dans les deux sous-corpus. Étant donné que le pronom on est souvent caractérisé comme la variante informelle de nous (cf. entre autres Coveney 2010: 134, le tableau (187) et la section 2.3.3), l'on pourrait être surpris par sa fréquence élevée dans la distance communicative. Néanmoins, vu que on s'emploie également comme pronom impersonnel stylistiquement neutre, sa présence élevée dans la distance communicative est compréhensible.

Étant donné que ce sont les mêmes variantes qui apparaissent dans les deux sous-corpus, la question qui se pose est de savoir comment ces données sont liées à l'hypothèse d'une diglossie.

S'il est correct de supposer que le sous-corpus de l'immédiat devrait représenter la grammaire 1, tandis que le sous-corpus de la distance contiendrait des énoncés générés par la grammaire 2, nous sommes amenés à conclure qu'il n'y a aucune caractéristique idiosyncratique qui permet de distinguer les deux grammaires, car presque toutes les variantes apparaissent dans les deux sous-corpus.

En résumant les résultats obtenus, les données dans notre corpus nous permettent de réfuter clairement l'hypothèse diglossique. Il semble plus convaincant d'admettre tout simplement qu'il existe une variation à l'intérieur de la grammaire du français et d'essayer d'expliquer cette variation en termes de régularités internes, par exemple comme nous le proposons dans les sections 5.2 et 5.3 ( $c f$. aussi Coveney 2011, Gadet 2007, Meisner 2013 et Rowlett 2013 pour davantage d'arguments empiriques et théoriques contre l'approche diglossique). 


\subsubsection{L'approche pragmatique: ne comme une particule emphatique}

En français québécois, la particule ne semble apparaître, depuis presque cent ans, seulement dans des contextes moralistes, sérieux ou emphatiques, c'est-à-dire dans des contextes pragmatiquement définis (cf. Poplack/St-Amand 2009, Sankoff/Vincent 1980 et section 2.2.5.7).

Les analyses qualitatives récentes des contextes $+n e$ montrent que l'idée d'une évolution de ne vers une particule pragmatique est également soutenue par un nombre croissant de corpus français hors du Québec. Ceci vaut par exemple pour le français en ligne (van Compernolle 2008a,b et section 2.2.1.2) ainsi que pour le français phonique informel de Suisse (Fonseca-Greber 2007) et de France (van Compernolle 2009). L'on pourrait donc éventuellement comparer ces variétés du français avec certains dialectes flamands qui ont développé une particule pragmatique à partir de l'ancienne particule négative en ( $c f$. Breitbarth/Haegeman à paraître et section 2.2.5.7).

Une remarque méthodologique est de mise ici: à la différence du français, qui est une langue standardisée et nationale avec une grande tradition littéraire, ces dialectes flamands ne sont pas normés et appartiennent surtout au domaine de l'immédiat. Ceci implique que les dialectes flamands permettent de tester l'existence de règles grammaticales qui exigent ou interdisent l'emploi de la particule en, tandis qu'il serait presque impossible de trouver un francophone prêt à confirmer l'agrammaticalité de la variante +ne dans une construction donnée, car en français standard l'emploi de ne est obligatoire ( $c f$. Coveney 1998 et Meisner 2013 pour des tentatives d'analyses de l'acceptabilité de $\pm n e$ dans divers contextes).

Pour vérifier l'hypothèse pragmatique, nous suivons donc la méthodologie établie par Fonseca-Greber (2007) et raffinée par van Compernolle (2009). Les deux contextes principaux pour + ne que FonsecaGreber (2007: 258-260) identifie sont: le ne micro-stylistique et le ne emphatique. Le premier terme désigne l'introduction de +ne comme marque stylistique formelle dans une conversation généralement plutôt informelle, déclenchée par l'évocation du 'monde institutionnel' (règles, lois, administration etc., cf. Fonseca-Greber 2007: 258). Le 
deuxième marque l'emphase en cooccurrence avec des traits phonologiques emphatiques, comme la répétition ou la combinaison d'un accent syllabique et une vitesse réduite, ce qui est confirmé par van Compernolle (2009):

(197) Indeed, the combination of slowed speech and syllable stress during a negation appeared to produce an environment that nearly categorically favored ne retention. (van Compernolle 2009: 11)

$\mathrm{Vu}$ que dans nos situations de distance, ce que Fonseca-Greber (2007) conçoit comme 'discours institutionnel' est omniprésent, il semble plus intéressant d'examiner les exemples + ne des situations de l'immédiat afin de tester d'éventuelles alternances du type micro-stylistique ou emphatique. Il s'agit d'un résidu relativement restreint qui figure en (198).

(198) Les exemples $+n e$ issus des situations de l'immédiat

\begin{tabular}{|c|c|c|}
\hline a. & 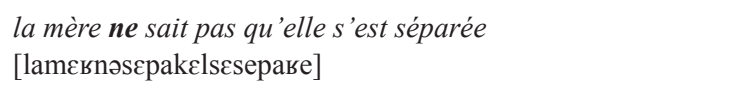 & 0796 \\
\hline b. & 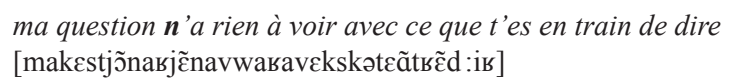 & 1248 \\
\hline c. & $\begin{array}{l}\text { je ne veux pas } \\
\text { [зənəvøpa] }\end{array}$ & 1244 \\
\hline d. & $\begin{array}{l}\text { ne repassez pas jamais } \\
\text { [nәьәраsepazam }]\end{array}$ & 2590 \\
\hline e. & 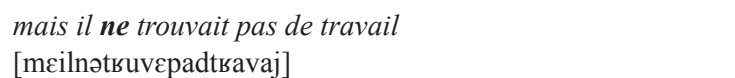 & 2276 \\
\hline f. & $\begin{array}{l}\text { ça ne me dérange pas } \\
\text { [sanmdекãzpa] }\end{array}$ & 1903 \\
\hline g. & $\begin{array}{l}\text { je ne sais pas } \\
\text { [зənsepa] }\end{array}$ & 2418 \\
\hline h. & $\begin{array}{l}\text { il ne va pas bien } \\
\text { [ilnəvapabjẽ }]\end{array}$ & 0862 \\
\hline
\end{tabular}

L'exactitude des prédictions de la théorie du ne-pragmatique est indiquée, comme dans la section précédente, par des symboles situés derrière les exemples en (198).

Les deux premiers exemples, en (198)a et b, ne laissent soupçonner aucune motivation pragmatique pour $+n e$. Au contraire, dans ces cas, 
il semble plus convainquant d'expliquer la présence de ne par le sujet grammatical lourd, qui, dans l'analyse de corpus, s'est avéré être le facteur le plus puissant conduisant à la réalisation de $n e$ ( $c f$. section 4.4.1.1). Les deux exemples suivants, (198)c et d, cependant, semblent être de bons candidats pour une explication emphatique de $+n e$.

(199) moi Pierre Boulle je veux pas / JE-NE-VEUX-PAS / je l'ai pas lu

En (199) figure le contexte direct de l'énoncé (198)c. La locutrice, une élève de la banlieue parisienne, se prononce sur ses préférences pour l'examen oral qui l'attend. Elle produit une série de trois énoncés négatifs, dont seul le deuxième comprend ne. Ce dernier est par ailleurs caractérisé par l'intonation staccato décrite comme typique des négations emphatiques par van Compernolle (2009: 11): chaque syllabe est accentuée, le débit de parole est réduit par rapport au reste de l'énoncé et les morphèmes sont séparés par des micropauses. L'exemple (198)c répété sous (199) contient donc clairement un ne emphatique selon les critères de van Compernolle (2009: 11).

Pour comprendre l'exemple suivant, (198)d, nous avons besoin de son contexte plus large, qui figure en (200).

(200) Le contexte de l'exemple (198)d (énoncé 2590)

A: j'ai mis trois mots quatre minutes / j'étais là comme ça tu vois [...]

B: elle va l'entendre RIRES elle va vous le repasser en cours pour bien vous torturer

A: c'est mort / ne repassez pas-jamais

L'élève A (la même qui produit l'exemple précédent (198)c) raconte comment s'est déroulé l'horrible examen oral qu'elle vient de passer. Sa camarade lui rappelle que l'examen a été enregistré et que elle, la chercheuse, pourrait faire repasser l'enregistrement en cours. A supplie ensuite la chercheuse de ne pas repasser l'enregistrement et ajoute après une micropause le deuxième élément négatif jamais. L'emploi insistant de pas et jamais à la fois en (198)d/(200) indique très clairement qu'il s'agit d'un emploi emphatique de ne dans ce cas aussi.

Les exemples suivants, (198)e à h, ne peuvent pas être identifiés comme des emplois emphatiques ou pragmatiquement motivés de ne. 
Pour (198)e, l'on pourrait à la rigueur supposer qu'il s'agit d'un discours 'institutionnel', puisque le sujet du travail est thématisé. Néanmoins, étant donné qu'il y a de nombreux exemples sans ne dans le corpus qui thématisent le même sujet, cette argumentation ne semble pas suffisante, comme le constate aussi Fonseca-Greber (2007: 259): «A public or institutional topic does not guarantee a micro-shift in register to include ne usage». Nous notons également que les exemples (198)e et $\mathrm{f}$ comportent un élément prosodiquement lourd (la conjonction mais et le pronom $c ̧ a$ ) dans le contexte gauche de la variable \pm ne. (La section 5.2.4 montrera pourquoi ceci peut être pertinent pour la réalisation de ne.)

Le dernier exemple, en (198)h, mérite d'être commenté. Il est produit lors d'une discussion métalinguistique concernant la construction du verbe allemand gehen 'aller', menée en alternance codique entre le français et l'allemand. L'assistante d'allemand (A) explique les pièges de cette construction, en se basant pour cela sur un texte allemand que l'élève (B) a sous les yeux.

(201) Le contexte de l'exemple (198)h (0862, allemand en caractères gras)

A: si on dit wie gehst du hein ich gehe gut - ich gehe schlecht ça veut dire geht ça se réfère seulement euh au verbe aller dans le sens devraiment de marcher hein - donc ça veut dire je marche mal RIRES je marche bien [...] dem geht's gut ça veut dire

B: je vais bien

A: oui - il va bien - il va bien / aber was sagt die Mutter / [NomPr] was sagt die Mutter hier - sie sagt / elle ne dit pas euh il va bien - mais...

B: il ne va pas bien

$\mathrm{A}$ : voilà...

L'énoncé 0862 est donc pratiquement la traduction d'une phrase tirée d'un texte écrit en allemand, un commentaire métalinguistique. Par conséquent, il n'est pas surprenant que la locutrice, une élève de la banlieue parisienne, ait recours à la forme normativement correcte, même si ni le contexte grammatical ni la situation le lui demandent.

Pour conclure cette section, nous pouvons retenir que les caractéristiques intonatives de l'emphase formulées par van Compernolle (2009) peuvent aider à identifier et expliquer certaines occurrences de +ne qui, autrement, resteraient inexplicables. Toutefois, la majorité des 
exemples rapportés ici échappe aux critères micro-stylistiques discutés ou pourrait tout aussi bien être expliquée par des facteurs linguistiques (le type de sujet, l'environnement phonologique etc.) ${ }^{140}$. Une analyse discursive prudente peut tout de même nous faire découvrir de nouveaux aspects intéressants de la variation autour de \pm ne et nous amener à une compréhension plus profonde des données, comme le dernier exemple l'a montré.

\subsubsection{L'approche du contact linguistique}

La dernière hypothèse évaluée ici a surgi de notre analyse de corpus (cf. section 4.3.4) et n'a, à notre connaissance, jamais été évoquée dans la littérature scientifique relative au présent sujet. Elle concerne l'interaction entre la généralisation de la négation monopartite et le statut bilingue des locuteurs. Nous nous demandons s'il est possible que les locuteurs bilingues, qui ne réalisent dans notre corpus aucun ne, généralisent la négation monopartite par analogie syntaxique (angl. syntactic borrowing) avec leurs deuxièmes langues maternelles.

Afin d'approfondir cette hypothèse, considérons la syntaxe négative dans les langues de contact attestées dans notre corpus.

(202) L'expression de la négation dans les langues de contact ( $c f$. Dryer 2011b)

\begin{tabular}{|llc|}
\hline Langue de contact & \multicolumn{2}{c|}{ Expression de la négation } \\
\hline $\begin{array}{l}\text { Anglais } \\
\text { Arabe }\end{array}$ & $\begin{array}{l}\text { particule négative postverbale } \\
\text { pégation bipartite optionnelle }\end{array}$ & VNEG \\
& NEGV/[NEG-V-NEG] \\
Suisse-allemand & particule négative postverbale & VNEG \\
Turc & affixe négatif postverbal & VNEG \\
\hline
\end{tabular}

140 Ceci vaut également pour la majorité des exemples cité par van Compernolle (2009). Ce que cet auteur appelle syllable stress coïncide très souvent avec des contextes intervocaliques de $n e$, connus pour favoriser sa réalisation. 
En effet, dans trois des quatre langues de contact, à savoir l'anglais, le suisse allemand et le turc, la négation est exprimée par un seul élément (particule ou affixe) postverbal (VNEG) :

(203) La négation postverbale dans les langues de contact

a. the mouse did not eat the cheese.

(anglais) (cf. Dryer 2011b)

b. chume hüt leider nöd (suisse-alémanique) ${ }^{141}$

venir-1Sg aujourd'hui malheureusement NEG

'malheureusement je ne viens pas aujourd'hui'

c. Oku-mu-yor-um

(turc) (cf. Dahl 2010: 14)

lire-NEG-PROG-1Sg

'je ne suis pas en train de lire'

En arabe, la situation est un peu plus complexe: Haspelmath et al. (2011) notent 21 variétés diatopiques de l'arabe. En arabe standard ainsi que dans les variétés orientales de la région du golfe, la négation préverbale (VNEG) est obligatoire, comme l'exemple (204) le montre.

(204) La négation préverbale dans les dialectes arabes orientaux

$\rightarrow$ par exemple dans l'arabe parlé au Koweït (cf. Brustad 2000: 280)

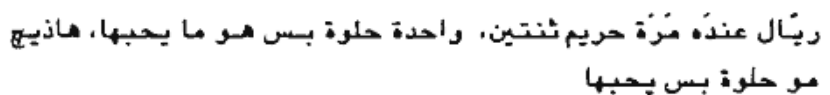

rayyāl cinda mara ḥarīm tintēn, waḥda ḥilwa bass hu mā yḥibbha hadīič mū hịilwa, bass yḥibbha man at-him woman wives two one pretty but he neg he-loves-her that-one neg pretty but he-loves-her A man has a wife, two wives, one is pretty but he doesn't love her, the other one is not pretty but he loves her

(205) La négation bipartite pré et postverbale dans les dialectes arabes occidentaux $\rightarrow$ par exemple en arabe marocain (cf. Brustad 2000: 279)

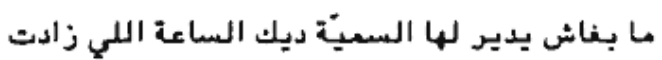

mā bğāš ydīr-lha s-smiyya dīk s-sāca lli zādət

neg he-wanted-neg make-for-her the name that the-hour that shewas-born

He didn't want to give her a name at the time she was born

$141 C f$. corpus $<$ sms 4 science.ch $>$, ID-SMS 825 . 
Dans les dialectes occidentaux du Maghreb (par exemple en marocain), la négation bipartite illustrée en (205) (NEGVNEG) est le procédé standard (cf. Brustad 2000: 277-314, Cowan 1958: 99 et Harrel 1962: 152). Malheureusement, il n'est plus possible de retracer la provenance géographique des familles des trois locuteurs franco-arabes dans notre corpus afin de savoir s'ils emploient une négation préverbale monopartite ou bipartite dans leurs autres langues maternelles. Néanmoins, nous savons que, contrairement aux autres langues de contact, l'arabe ne présente jamais une négation postverbale unique.

Dans l'ensemble, l'on peut se demander si l'hypothèse du contact linguistique permet d'expliquer le fait que tous les bilingues dans le corpus sont des locuteurs du type B ne réalisant que la particule négative postverbale.

Quant aux bilingues parlant le français en plus de l'allemand, l'anglais ou le turc, il semble plausible qu'ils généralisent la négation monopartite de leurs deuxièmes langues maternelles, vu qu'en français deux stratégies sont admises. Toutefois, les trois locuteurs franco-arabes, qui dans leur deuxième langue emploient une négation préverbale ou bipartite, seraient censés, selon l'hypothèse du contact linguistique, généraliser la variante bipartite. Un deuxième argument contre l'hypothèse du contact linguistique est le fait que le type B (i.e. les locuteurs sans ne) comprend non seulement des locuteurs bilingues, mais également des locuteurs monolingues. L'hypothèse du contact linguistique n'offre donc qu'une explication partielle des faits observés. Néanmoins, une piste prometteuse pour de futurs travaux de recherche a été découverte. Une étude ciblée et approfondie de l'interaction entre le bilinguisme et la variation $\pm n e$ serait particulièrement souhaitable.

\subsubsection{Conclusion intermédiaire}

Dans les sections précédentes, nous avons vu que les cinq approches de la variation \pm ne offrent des explications prometteuses mais seulement partielles de la variation observée. 
Premièrement, la conception de \pm ne comme variable sociolinguistique ( $c f$. Armstrong/Smith 2002: 23, Coveney 22002: 55, Gadet 1997b, Labov 1972) ne reflète pas la réalité de notre corpus, dans lequel le francophone moyen sans spécification sociodémographique quelconque montre les deux variantes +ne et -ne, généralement en fonction des facteurs linguistiques. Deuxièmement, l'incompatibilité entre ne et d'autres clitiques, proposée par Ashby (1977), Culbertson/ Legendre (2008), Larrivée (2014) et Posner (1985: 189) n'est qu'une forte tendance dans le corpus, mais n'a rien de régulier et ignore complètement le caractère continuel du paradigme dit 'clitique' ( $c f$. section 2.2.5.2). Troisièmement, l'approche diglossique, qui soutient que les francophones vivraient entre deux grammaires, le français standard et le français de l'immédiat ( $c f$. Culbertson 2010, Massot 2010, Mensching 2008, Koch/Oesterreicher ${ }^{2} 2011$ et Zribi-Hertz 2011), manque de preuves empiriques et semble avoir été construite ad hoc dans le but, justemment, d'expliquer des phénomènes variationnels comme celui de $\pm n e$. Quatrièmement, l'approche pragmatique, qui soutient que ne serait désormais devenu une particule pragmatique ou d'emphase ( $c f$. van Compernolle 2008a,b, 2009, Fonseca-Greber 2007, Poplack/St-Amand 2009, Sankoff/Vincent 1980) comparable à en en flamand de l'ouest (cf. Breitbarth/Haegeman à paraître), offre des perspectives d'analyse qualitative ponctuelles de certains exemples, mais aucunement une explication globale de l'absence et de la présence de ne. Finalement, le statut bilingue des locuteurs comme possible déclencheur de l'absence totale de ne en français, découvert par hasard dans notre analyse de corpus, devrait être examiné en profondeur avant de pouvoir affirmer qu'il s'agit d'une explication valable de la variation $\pm n e$.

$\mathrm{Vu}$ les difficultés à trouver une explication globale de la variation $\pm n e$, nous allons (ré)examiner dans le prochain sous-chapitre un facteur dont l'importance pour \pm ne est bien connue (cf. Ashby 1976: 128, 1981 : 677, Le Bidois/Le Bidois 1938: 655, Lüdicke 1982: 45, Coveney ${ }^{2} 2002$ : 77-78), mais qui a été quelque peu négligé dans les analyses de corpus et qui reste donc jusqu'à présent exclu des explications théoriques de cette variable: il s'agit de l'influence de la prosodie. 


\subsection{Proposition d'une explication prosodique de $\pm n e$ en français}

\subsubsection{Pourquoi une explication prosodique?}

Dès les premiers travaux sur le phénomène de variation $\pm n e$ de Jespersen (1917, 1924), la faiblesse prosodique de ne a été associée avec sa disparition, comme la citation de Le Bidois/Le Bidois (1938) en (206) témoigne.

(206) La négation ne, étant à la fois monosyllabique et proclitique, se réduit ainsi fréquemment à la seule lettre $n[\ldots]$. N'ayant plus de voyelle sonore où s'appuyer, l'n [sic] tombe à son tour et d'autant plus facilement que l'auxiliaire de négation. (Le Bidois/Le Bidois 1938: 655)

Dans cette perspective et dans celle diachronique et typologique adoptée par Jespersen $(1917,1924)$, la faiblesse de ne constitue l'explication par excellence au besoin de le renforcer par d'autres éléments et à sa disparition successive (cf. Jespersen 1992 [1924]: 479).

Cependant, dans une perspective synchronique variationnelle, le système prosodique du français ( $c f$. section 5.2.2) et son rôle pour la variable $\pm n e$ restent, à notre connaissance, exclus des explications théoriques décrites en section 2.4 , et ce même si le contexte phonétique de $\pm n e$ et le débit de parole s'avèrent empiriquement pertinents ( $c f$. Ashby 1976: 128, 1981: 677, Coveney 22002: 77-78, Lüdicke 1982: 45). Nous nous proposons donc de montrer, par la suite, qu'une explication prosodique de $\pm n e$ émerge tout naturellement d'une évaluation prudente de l'ensemble des données empiriques disponibles.

La prosodie est tout d'abord un 'métafacteur' qui intègre les facteurs linguistiques qui se sont avérés pertinents pour la réalisation de ne dans l'analyse multifactorielle ( $c f$. sections 4.5 .1 et 5.2.4), c'est-à-dire l'effet des sujets lourds, légers et redoublés et des séquences proclitiques ainsi que celui des principales et des subordonnées. De plus, même le type de situation communicative, le seul facteur extralinguistique statistiquement significatif dans l'analyse multifactorielle, se laisse inté- 
grer dans l'approche prosodique: ceci se fait à travers la fréquence des éléments linguistiques pertinents pour $\pm n e$, qui varie considérablement en fonction de la situation ( $c f$. section 5.3). Finalement, l'approche proposée ici permet l'intégration de certaines lignes argumentatives provenant d'autres approches de $\pm n e$ ( $c f$. section 5.4.2).

Ainsi, une évaluation prudente du facteur prosodique permet l'explication de tous les exemples négatifs du corpus et l'intégration de bon nombre d'explications proposées jusqu'à présent dans la littérature, ce que les approches alternatives évaluées dans la section 5.1 n'ont pas réussi à faire.

\subsubsection{La prosodie du français}

Toutes les descriptions de la prosodie du français assument un accent primaire final, assigné à la dernière syllabe pleine (i.e. sans schwa) d'un groupe accentuel. Ce groupe sera désigné par la suite comme AP («accentual phrase», cf. Jun/Fougeron 2000: 210 aussi «stress group» Di Cristo 1998: 196 ou «prosodic phrase» Welby 2003: 10).

Contrairement à d'autres langues romanes ( $c f$. it. anCOra [aך'ko :ra] 'encore' vs. ANcora ['anko:ra] 'ancre'), l'accent principal du français n'a aucune fonction distinctive. Il est assigné selon trois principes ( $c f$. Di Cristo 1998: 196 suivant Garde 1968): d'abord, le principe de l'accentogénéité, qui effectue la sélection des éléments accentuables (généralement des mots lexicaux), ensuite le critère du groupage, qui unit les éléments lexicaux aux clitiques qui l'accompagnent, et finalement le principe de la tête à droite, qui assigne l'accent à la dernière syllabe accentuable du groupe. Les deux derniers principes expliquent pourquoi les clitiques, même s'ils sont généralement inaccentués, peuvent, en position finale, porter un accent:

(207) Crois-TU |que c'est BIEN?

(cf. Di Cristo 1998: 196)

Si un groupe accentuel se trouve en position finale dans un groupe intonatif, l'accent final coïncide avec le contour tonal croissant ou caduc de l'intonation. 
Par ailleurs, la plupart des chercheurs soutiennent que le français possède un accent initial optionnel, désigné par la suite comme Hi (cf. «initial high tone», Jun/Fougeron 2000: 210, aussi «accent initial», «accent secondaire» ou «ictus mélodique», Jun/Fougeron 2000: 211), qui apparaît «near the beginning of the phrase» (Welby 2003: 11).

Contrairement à l'accent final, l'accent initial n'apparaît pas dans tous les groupes accentuels. Il semble y avoir un certain consensus sur le fait que l'accent initial se place sur l'une des premières syllabes du premier mot lexical des groupes accentuels polysyllabiques. Jun/Fougeron (2000) notent à cet égard:

(208) [...] we noticed that the realization of $\mathrm{Hi}$ is sensitive to the presence of a function word. When an AP begins with one or more function words, especially when the function words are monosyllables, Hi tends to be realized after all the function words. (Jun/Fougeron 2000: 212)

Hi est donc réalisé au début de la phrase uniquement sur les morphèmes lexicaux et non pas sur les proclitiques grammaticaux, qui, comme nous l'avons vu dans la section 2.2.5.2, sont inaccentuables. La sensibilité de l'accent initial à l'information lexicale a déjà été observée par Hirst/Di Cristo (1996), qui montrent que Hi est lié au début d'un mot lexical, et par Vaissière (1997), qui propose que l'accent initial se situe sur la première ou la deuxième syllabe du premier mot lexical.

Dans les groupes accentuels lexicaux, l'accent initial est réalisé sur la première ou la deuxième syllabe, comme les résultats d'un travail de Jun/Fougeron (2000: 211-212) sur la prononciation des lexèmes dis- et polysyllabiques (comme par exemple in-vite, in-vi-ter, in-di-vi-du, in$d i$-vi-du-a-lisme, in-di-vi-du-a-li-té, in-di-vi-du-a-li-sa-tion) en (209) le montrent. 
(209) La position de l'accent initial dans les AP lexicaux de 2 à 8 syllabes
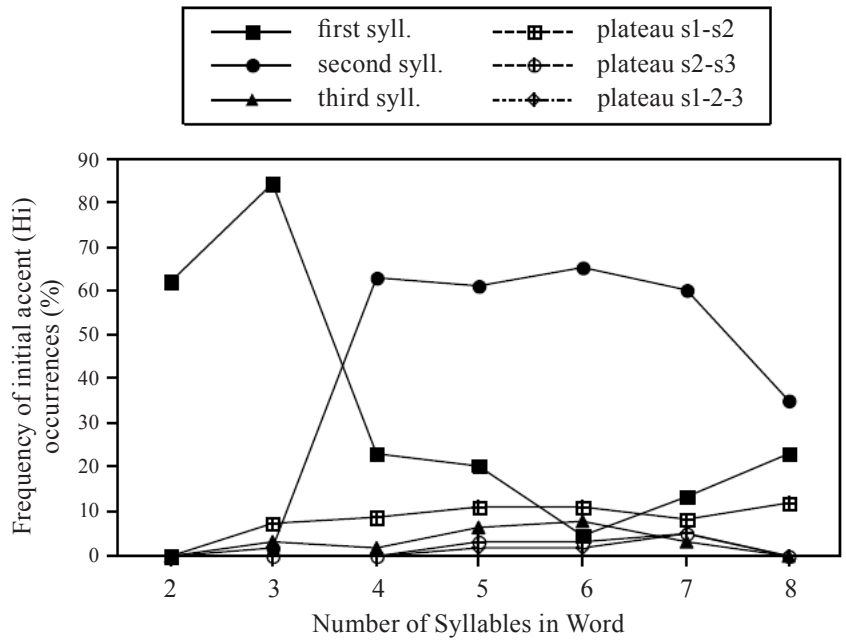

Location of initial accent (Hi) within a 2-8 syllable-word which forms an AP in sentence initial position: the frequency (in \%) of realization of $\mathrm{Hi}$ on the 1st, 2nd, 3rd syllable, or as a plateau high realized over the initial two or three syllables (e.g., "plateau s1-s2": Hi over the 1st and 2nd syllables).

Le diagramme (209) montre que dans les lexèmes dis- et trisyllabiques, comme invite et inviter, l'accent initial est généralement placé sur la première syllabe. Dans les lexèmes de quatre syllabes ou plus (par exemple. individu, individualisme, individualité et individualisation), Hi se situe sur la deuxième syllabe.

Contrairement à sa position, les fonctions de l'accent initial ne sont pas encore complètement explorées. Il semble clair que l'accent initial ne doit pas être confondu avec l'accent emphatique ( $c f$. c'est DEgeulasse, Di Cristo 1998: 198), même s'il apparaît dans la même position. Son absence ou présence ne semble pas entraîner une différence pragmatique, mais Fónagy (1979) note tout de même qu'il forme avec l'accent final un arc accentuel qui facilite la reconnaissance de l'unité sémantique du groupe. Welby (2003: 61-223) confirme dans une expérience perceptuelle que l'accent initial sert à la segmentation de la chaîne parlée et à la reconnaissance du début d'un mot lexical. Astésano/Bard (2003) constatent qu'il marque au niveau rythmique et 
structurel la frontière gauche d'un groupe accentuel et qu'il peut servir comme ressource de désambiguïsation prosodique.

$\mathrm{Au}$ niveau phonétique, aussi bien l'accent initial que l'accent final se manifestent par une proéminence tonale. Selon Di Cristo (1998: 199), l'accent final montre également des signaux temporels comme le prolongement de la syllabe portant l'accent et la réduction de la syllabe suivante. Welby (2003: 11) note que l'accent initial peut être lui aussi accompagné d'un allongement syllabique et d'une intensité élevée. Astésano/Bard (2003 : 503) soutiennent que l'accent initial se distingue phonétiquement de l'accent final par l'allongement de l'attaque de la syllabe (au lieu de la rime).

Même si l'accent initial est aujourd'hui complètement intégré dans le système prosodique du français, il semble être un phénomène relativement récent dans l'évolution de cette langue. Selon Welby (2003 : 13), il a été condamné jusqu'au début du $19^{\mathrm{e}}$ siècle par les grammairiens, qui le considéraient comme atypique du français ou comme une émanation du français populaire ( $c f$. Delattre 1940). De nos jours, il est cependant observable dans toutes les situations de communication, également dans la distance communicative ( $c f$. Welby 2003: 14).

\subsubsection{Le modèle prosodique de Jun/Fougeron (2000)}

L'argumentation qui suit se basera surtout sur l'accent initial et sa sensibilité à la nature grammaticale ou lexicale des morphèmes qui peuvent le porter. Nous allons plus précisément argumenter que l'omission fréquente de la particule ne avec les sujets clitiques est due à une tendance de compensation prosodique. Comme les séquences proclitiques ne peuvent pas porter l'accent initial, ils vont à l'encontre du principe eurythmique. Selon ce principe, évoqué par Dell (1984), le français favorise l'alternation des syllabes fortes et faibles afin d'éviter des séquences rythmiquement déséquilibrées ( $c f$. Di Cristo 1998: 197). À travers l'omission du ne, une séquence clitique peut être réduite et le groupe accentuel mieux équilibré. 
Cette idée sera explicitée à l'aide d'un modèle prosodique de Jun/ Fougeron $(1995,2000,2002)$ et Fougeron/Jun (1998) ${ }^{142}$. Il s'agit d'un modèle développé dans le cadre de la théorie autosegmentale-métrique selon Pierrehumbert (1980), qui formalise les tendances intonatives décrites dans la section 5.2.2 ci-dessus. Jun/Fougeron (2000) décrivent l'intonation à l'aide des tons discrets (haut: $\mathrm{H}$, bas: L, accent:*, frontière: \%), situés sur une couche tonale autonome et associés aux syllabes fortes $\left(\sigma_{\mathrm{s}}\right)\left(c f\right.$. angl. CHOC(o)late ['t $\mathbf{f} \mathbf{p} \mathbf{k}_{\sigma \mathrm{s}}$. lət] vs. all. SchokoLAde,

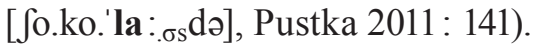

En français, l'accent principal final $\left(\mathrm{H}^{*}\right)$ est associé à la dernière syllabe d'un groupe accentuel et l'accent secondaire initial (Hi) tombe sur la première ou la deuxième syllabe. Le contour intonatif basique d'un AP en français est donc: /LHiLH*/ (cf. Jun/Fougeron 2000: 210). Plusieurs APs forment une unité d'intonation (IP), qui est délimitée par un ton de frontière (\%) ( $c f$. Jun/Fougeron 2000: 210). La structure modèle d'un AP figure en (210).

(210) La structure intonative d'un AP selon Jun/Fougeron (2000: 214)

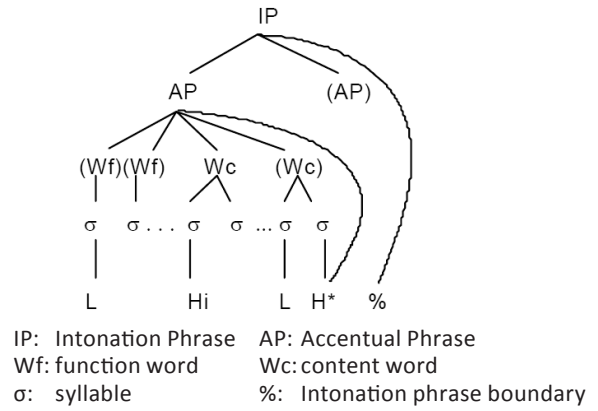

Schematics of underlying tones in AP (LHiLH*) and their affiliation.

$142 C f$. Welby (2003: 26-60) pour une vue d'ensemble des modèles prosodiques du français et pour une discussion de Jun/Fougeron (2002). Welby (2003 : 51-52) résume une série de critiques à ce modèle, entre autres, l'assomption d'une structure basique de quatre tons, qui serait trop restrictive, et du contour basique /LHiLH*/, auquel toutes les autres formes observées sont attribuées comme des variantes. Toutefois, malgré la critique, Welby (2003 : 72) adopte également le modèle de Jun/ Fougeron (2002) comme point de départ de sa propre analyse, notamment à cause de son inventaire terminologique très précis et ses prédictions théoriques claires. 
Jun/Fougeron (2000: 214) assument l'existence de quatre tons basiques associés à un AP. La réalisation concrète des accents varie en fonction du nombre et de la nature (grammaticale ou lexicale) des morphèmes dans un AP, du débit de parole et du style individuel du locuteur.

L'AP peut être monosyllabique (par exemple $\mathrm{Paul}$ ) ou comporter jusqu'à environ huit syllabes (par exemple in-di-vi-du-a-li-sa-tion). Dans un AP monosyllabique, seul $\mathrm{H}^{*}$ est réalisé. Pour les autres types d'AP, Jun/Fougeron (2000: 216) proposent cinq réalisations concrètes du contour intonatif abstrait /LHiLH*/:

(211) Cinq réalisations possibles de AP /LHiLH*/ selon Jun/Fougeron (2000: 216)

Five types of surface realizations of AP (/LHiLH*/) when not all four underlying tones are realized. The tone(s) in a parentheses refers to the tone(s) not realized due to undershoot.

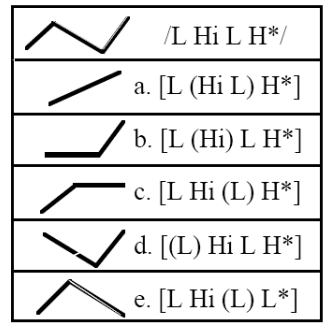

En règle générale, si l'AP se compose d'au moins quatre syllabes, les quatre tons sous-jacents sont réalisés. En (211)a-e figurent les réalisations alternatives qui se manifestent si certains tons abstraits ne sont pas réalisés ( $c f$. «undershot», Jun/Fougeron 2000: 216). Les auteurs observent le contour (211)a [LH*] pour les AP mono- ou dissyllabiques: $P A U L_{\mathrm{H}^{*}}$ ou $m e ́ D I T E_{\mathrm{H}^{*}}$. Pour les trisyllabiques, il y a quatre possibilités différentes: (211)a [LH*], (ex. mé(di)TER $\left.\mathrm{H}^{*}\right) \mathrm{b}$ [LLH*] (ex. médiTER), c [LHiH*] (cf. méDI $\left.I_{\mathrm{Hi}} T E R_{\mathrm{H}^{*}}\right)$ ou d [HiLH*] (ex. $\left.M E_{\mathrm{Hi}} d i T E R_{\mathrm{H}^{*}}\right)$. Finalement, un AP polysyllabique peut également montrer le contour en (211) e [LHi(L)L*] si l'AP est suivi par un autre AP qui commence en Hi (ex. $\left.m e ́ D I_{\mathrm{Hi}} t a T I O N_{\mathrm{L}^{*}} I N_{\mathrm{Hi}} i T I A L E_{\mathrm{H}^{*}}\right)$ ou s'il est le dernier AP dans un IP qui se termine en $\mathrm{L} \%$ (ex. j'aDORE $\mathrm{H}^{*}$ la $\left.M E_{\mathrm{Hi}} \operatorname{ditaTION}_{\mathrm{L} \%}\right)$. 


\subsubsection{Application $\grave{a} \pm$ ne}

\subsubsection{L'influence prosodique des sujets légers}

La réalisation et la position de l'accent initial Hi dépendent non seulement de la longueur d'un AP, mais également de sa composition en termes d'unités grammaticales et lexicales. Contrairement aux AP lexicaux, dans les AP qui commencent sur un ou plusieurs morphèmes grammaticaux (comme les clitiques sujet, objet ou de négation), sur lesquels Hi ne peut pas être réalisé, l'accent tombe obligatoirement sur la première syllabe du premier élément lexical qui est, dans les cas qui nous intéressent ici, généralement le verbe, $c f$. (212).

(212) Les positions de Hi (en gris) dans les AP lexicaux et grammaticaux (cf. Jun/Fougeron 2000: 21) $)^{143}$

\begin{tabular}{|c|c|c|c|c|c|c|c|c|}
\hline & 1 & 2 & 3 & 4 & 5 & 6 & 7 & 8 \\
\hline & $\sigma$ & $\sigma$ & $\sigma$ & $\sigma$ & $\sigma$ & $\sigma$ & $\sigma$ & $\sigma$ \\
\hline \multirow{7}{*}{$\begin{array}{l}\text { a. APs lexicaux: } \\
\text { Hi sur la } 1^{\mathrm{e}} \text { ou } 2^{\mathrm{e}} \text { syllabe de } \\
\text { l'AP }\end{array}$} & $m e ́$ & dite & & & & & & \\
\hline & $m e ́$ & $\underline{d i}$ & ter & & & & & \\
\hline & mé & $d i$ & $t a$ & tion & & & & \\
\hline & mé & $d i$ & ter & $r a$ & née & & & \\
\hline & mé & $d i$ & ter & $\mathrm{ra}$ & $n e ́$ & en & & \\
\hline & mé & $d i$ & ter & $r a$ & $n e ́$ & $i$ & ser & \\
\hline & mé & $d i$ & ter & $\mathrm{ra}$ & né & $i$ & $s a$ & tion \\
\hline \multirow{7}{*}{$\begin{array}{l}\text { b. APs débutant en clitiques } \\
\text { grammaticaux: } \\
\text { Hi sur la } 1^{\mathrm{e}} \text { syllabe de } \\
\text { l'élément lexical dans l'AP }\end{array}$} & $\mathrm{Je}$ & veux & & & & & & \\
\hline & & veux & nas & & & & & \\
\hline & $\mathrm{Je}$ & $n e$ & veux & pas & & & & \\
\hline & $\mathrm{Je}$ & $n e$ & le & rends & pas & & & \\
\hline & $\mathrm{Je}$ & $n e$ & le & ren & voie & pas & & \\
\hline & $\mathrm{Je}$ & ne & le & lui & ren & voie & pas & \\
\hline & $J e$ & ne & le & lui & $r a$ & con & te & pas \\
\hline
\end{tabular}

143 Les positions de Hi dans les AP en (212)a ont été établies par Jun/Fougeron (2000: 211-212). Les positions de Hi dans les exemples en (212)b suivent les observations de Hirst/Di Cristo (1996), Vaissière (1997) et Jun/Fougeron (2000: 212), qui soutiennent que les proclitiques sont inaccentuables. 
La comparaison schématique des positions de Hi en (212) montre que l'accent initial (en gris) tombe sur la première ou la deuxième syllabe d'un AP lexical en fonction de sa longueur, comme désigné en (212) a. Par contre, dans les APs qui débutent par une séquence de clitiques, comme en (212)b, sa position est surtout conditionnée par les propriétés syntactico-prosodiques des syllabes contenues dans l'AP: vu que les clitiques grammaticaux sont inaccentuables, Hi devrait tomber par défaut sur la syllabe initiale du verbe, $c f$. (212)b. Par conséquent, un nombre croissant de clitiques préverbaux implique un déplacement à droite de l'accent initial jusqu'à la 5e syllabe. Il n'y a donc qu'une seule réalisation parallèle de $\mathrm{Hi}$, désignée par le cadre pointillé en (212), entre les deux types d'AP en (212)a et b: celle sur la deuxième syllabe. Seul l'AP dans lequel le clitique négatif ne est omis en (212)b manifeste alors le même contour intonatif que les APs lexicaux en (212)a.

Si nous partons du fait que le contour intonatif assumé en (210) par Jun/Fougeron (2000: 214) présente la structure prosodique générale du français (et non seulement celle des APs lexicaux ou comportant un seul clitique), il est surprenant que les APs contenant des proclitiques en (212)b se distinguent autant des APs lexicaux en (212)a quant à la position de Hi. De plus, le contour intonatif des APs comportant plus d'un proclitique (schématisé en (212)b) entraîne une série d'inconvénients prosodiques qui contredisent le principe eurythmique de Dell (1984):

(213) Inconvénients prosodiques des APs comportant une séquence de proclitiques

a. Difficultés articulatoires d'une série de syllabes inaccentuées

b. AP déséquilibré: syllabes inaccentuées à gauche vs. accumulation des tons à droite

c. Rapprochement défavorable ( $c f$. accent clash) de l'accent initial Hi en direction de l'accent principal final $\mathrm{H}^{*}$

Nous montrerons, par la suite, que les différences entre les deux types d'APs en (212)a et b aussi bien que les inconvénients prosodiques des APs comportant des proclitiques, listés en (213), peuvent être surmontés par une série de stratégies de compensation, entre autres par l'omission du ne: 
(214) Stratégies de compensation dans les APs comportant plus d'un proclitique

a. Omission du ne (= syncope d'une syllabe atone sémantiquement superflue)

b. Réduction phonétique des proclitiques (= omission du schwa/d'autres voyelles, dévoisement)

c. Fusions entre clitiques (ex. $\operatorname{cod}+\mathrm{coi}: l e+l u i>l u i)$ ou clitique et verbe

Ceci revient à dire que la structure intonative proposée par Jun/Fougeron (2000) en (210) vaut pour tous les APs du français et tend possiblement à être réalisée dans tous les contextes grammaticaux du français: nous partons alors de l'idée qu'il y a une forte tendance à réaliser $\mathrm{Hi}$ sur l'une des premières syllabes d'un AP. Ce postulat de base permet d'expliquer et de réinterpréter les phénomènes de variation linguistique observés en français moderne, listés en (214), comme des stratégies de compensation prosodique.

La première stratégie compensatrice est l'omission de la particule de négation ne, dont l'effet est illustré en (215), ce qui permet un rapprochement entre les structures contenant des proclitiques et le contour prosodique des APs soutenu par Jun/Fougeron (2000) ${ }^{144}$.

(215) L'omission de ne comme stratégie de compensation prosodique

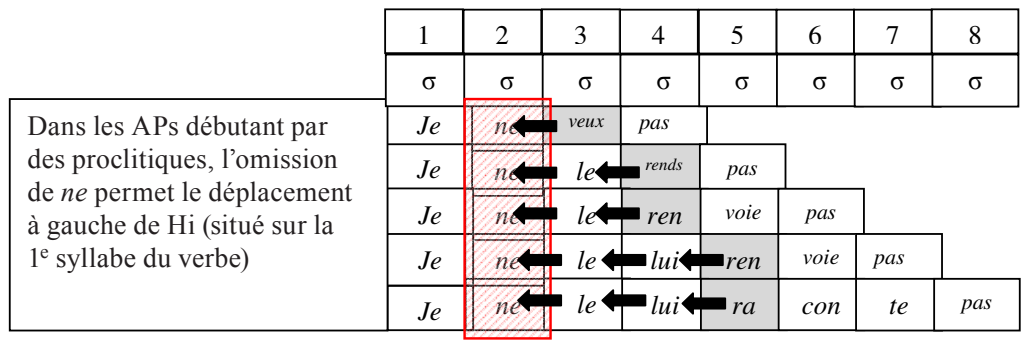

Comme la figure en (215) le montre, l'accent initial se déplace vers le début du groupe accentuel si ne est omis. Cette position sur la deuxième syllabe est le lieu naturel de $\mathrm{Hi}$, ce qui fait qu'en présence d'un ou de plusieurs autres proclitiques (marquant le sujet, le COD, COI etc.), les structures sans $n e$ sont clairement favorisées.

144 Les autres stratégies de compensation prosodique, qui seront discutées en détail dans la section suivante, ont le même effet que l'omission de $n e$ : elles provoquent elles aussi le déplacement de Hi à gauche. 
En conlusion, nous constatons que l'accent initial Hi est réalisé de préférence sur l'une des premières syllabes d'un groupe accentuel. Ce fait est indépendant de la structure interne de l'AP et a été prédit par de nombreux experts de la phonologie française ( $c f$. Jun/Fougeron 1995, 2000, 2002, Di Cristo 1998, Astésano/Bard 2003, Welby 2003). Dans les APs comportant des proclitiques, l'omission du ne et d'autres stratégies de compensation prosodique (décrites dans la section suivante) soutiennent la réalisation de Hi dans cette position initiale naturelle, ce qui n'est pas nécessaire dans les APs lexicaux ( $c f$. section 5.2.4.3). Plusieurs analyses de corpus (y compris la nôtre, $c f$. sections 4.4.1 et 4.5.1) ont prouvé statistiquement que l'omission de ne est provoquée en premier lieu par les sujets clitiques légers. Nous avons vu que ceci peut être expliqué par les stratégies de compensation prosodique conduisant à la réduction des séquences proclitiques, par exemple à travers l'omission de ne. Dans ce sens, la variation $\pm n e$ est un épiphénomène des caractéristiques prosodiques du type de sujet employé dans l'énoncé négatif.

\subsubsection{L'influence prosodique des séquences proclitiques}

Après avoir montré que l'omission de ne peut en effet être considérée une stratégie de compensation prosodique, nous examinons, par la suite, une deuxième stratégie de compensation, illustrée en (216), qui concerne non seulement le clitique négatif ne, mais tous les clitiques préverbaux.

(216) La réduction des proclitiques comme stratégie de compensation prosodique

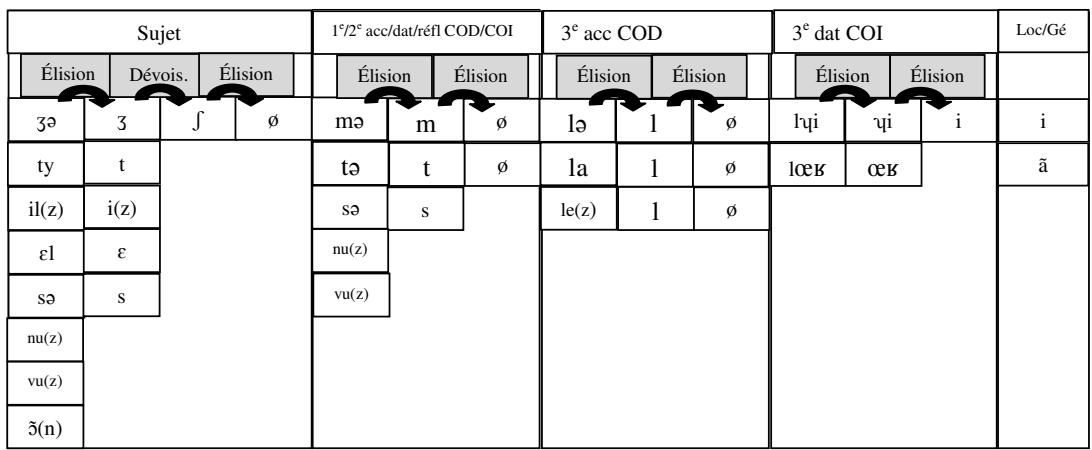


En (216) figurent les proclitiques français (sans ne) dans l'ordre de leur occurrence en position préverbale ( $c f$. section 2.2.5.3). Les formes des pronoms personnels sont présentées verticalement et les différentes variantes attestées pour chaque pronom figurent dans des cases individuelles à côté de celles-ci. Au-dessus des formes, les flèches et les cases grises indiquent les processus phonologiques qui opèrent entre les différentes formes d'un clitique. Il s'agit presque toujours d'élisions et, dans le cas de [3] $\rightarrow$ [J], d'un dévoisement, qui est observé devant les verbes en attaque sourde (ex. je pense [ jpãs]).

Tous les processus illustrés en (216) conduisent à une érosion phonétique des clitiques, les réduisent souvent à une seule consonne et les privent ainsi de leur nature syllabique. Comme effet secondaire de ces processus, nous pouvons noter que l'élision des voyelles conduit (surtout dans les séquences polyclitiques) à des attaques syllabiques complexes et défavorisées (je le dis $\rightarrow$ [3ldi]). Ces attaques complexes tendent à être réduites, ce qui provoque à son tour une élision des consonnes représentant un clitique $(\rightarrow[3 \mathrm{di}])$. L'omission des clitiques le, la, les devant lui et leur est décrite comme un processus d'hapologie ( $c f$. aussi Pomino 2009), ce qui signifie qu'il s'agit d'un cas particulier de dissimilation, donnant lieu à l'élision d'une syllabe adjacente à une unité similaire (par exemple all. *Zaubererin $\rightarrow$ Zauber_in, $c f$. Bussmann ${ }^{4} 2008$ : 255).

Les omissions de clitiqueset les fusions entre plusieurs clitiques ou entre un clitique et un verbe, dont certaines sont déjà presque lexicalisées ( $c f$. je sais pas $\rightarrow\left[\int \varepsilon \mathrm{pa}\right]$ ), ont le même effet que les autres processus de compensation, c'est-à-dire qu'elles permettent le déplacement de Hi à gauche:

(217) Les fusions clitique+clitique ou clitique+verbe comme stratégies de compensation prosodique (la syllabe qui peut porter Hi est soulignée)

a. Je le lui ai dit [3 Yicdi]

b. Je le leur ai dit [зœкеdi]

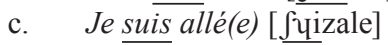

d. Je sais pas [jepa]

e. Il $y \underline{a}[\underline{\mathrm{ja}}]$ 
L'ensemble des stratégies de compensation réduit donc la périphérie gauche d'un AP contenant des proclitiques et le rapproche prosodiquement des APs lexicaux. Ceci signifie que seule la réduction des proclitiques rend possible la réalisation de Hi sur une des premières syllabes.

Or, nous avons vu lors de l'analyse de corpus ( $c f$. section 4.4.2) que l'influence des proclitiques sur $\pm n e$ est quelque peu paradoxale: la présence d'un clitique sujet léger conduit très probablement à l'omission de $n e(6 \%+n e)$, tandis que la présence de plusieurs clitiques produit un taux de ne un peu plus élevé $(14 \%+n e)$. Il faut alors se demander comment ce résultat s'accorde avec l'explication prosodique.

Nous avons proposé dans la section 4.4.2 que les réalisations de $n e$ dans les séquences proclitiques sont tout simplement des hypercorrections, puisqu'elles se trouvent exclusivement dans la distance communicative.

Afin de déterminer si cette hypothèse permet d'expliquer les exceptions au principe prosodique que nous revendiquons, toutes les séquences proclitiques qui pourraient potentiellement comprendre la particule ne seront analysées d'un point de vue phonotactique. Les colonnes dans le tableau (218) correspondent à l'ordre grammatical des clitiques préverbaux du français ( $c f$. section 2.2.5.4). La première colonne du tableau (218), celle du sujet, est toujours remplie. Dans la deuxième colonne figure, dans les rares cas de sa présence, le clitique de négation ne. Les colonnes suivantes sont remplies selon les données que nous avons trouvées dans le corpus: dans l'avant-dernière colonne figurent les réalisations phonétiques de chaque séquence clitique et la dernière colonne montre les structures syllabiques respectives. 
(218) Les séquences proclitiques $\pm n e$ et leurs réalisations dans le corpus ${ }^{145}$

\begin{tabular}{|c|c|c|c|c|c|c|c|c|}
\hline 1 & 2 & 3 & 4 & 5 & 6 & 7 & \multirow[t]{2}{*}{ réalisation } & \multirow{2}{*}{$\begin{array}{l}\text { structure } \\
\text { syllabique }\end{array}$} \\
\hline nom & 'nég' & réfl/acc/dat & $3^{\mathrm{e}}$ acc. & $3^{\mathrm{e}}$ dat. & loc. & gén. & & \\
\hline je & & $m e$ & & & & & [3m], [3m॰] & $\mathrm{CC}, \mathrm{CCV}$ \\
\hline je & & te & & & & & {$\left[\int \mathrm{ft}\right]$} & $\mathrm{CC}$ \\
\hline je & & & le & & & & [3lə], [3əl] & $\mathrm{CCV}, \mathrm{CVC}$ \\
\hline je & & & la & & & & [3ə.la], [3la] & CV.CV, CCV \\
\hline je & & & $l$ & & & & {$[31]$} & $\mathrm{CC}$ \\
\hline je & & & & lui & & & [3lui] & CCglideV \\
\hline$j^{\prime}$ & & & & & & en & [3ã] & $\mathrm{CV}$ \\
\hline$t u$ & & & & & & en & [tã] & $\mathrm{CV}$ \\
\hline il & $n^{\prime}$ & & & & $y$ & & [il.nj] & VC.Cglide \\
\hline il & & me & & & & & [il.mə] & VC.CV \\
\hline il & & se & & & & & [il.sə] & VC.CV \\
\hline il & ne & $s^{\prime}$ & & & $y$ & & [il.nə.si] & VC.CV.CV \\
\hline$i l$ & & & le/ l' & & & & [i.lə], [il(1)], & V.CV \\
\hline il & & & & lui & & & [i.lui] & V.CglideV \\
\hline il & & & & & $y$ & & [ilj], [j] & VCglide, glide \\
\hline elle & & se & & & & & [El.sə] & VC.CV \\
\hline elle & & nous & & & & & [El.nu] & VC.CV \\
\hline elle & & & & & & en & [عl.ã] & VC.V \\
\hline on & ne & & $l^{\prime}$ & & & & [ว̃n.1] & VC.C \\
\hline on & & & la & & & & [̃̃.1a] & C.CV \\
\hline
\end{tabular}

Dans cette vue d'ensemble, nous observons très bien les processus de compensation prosodique qui ont été décrits ci-dessus: tout d'abord, nous notons que les séquences de 2 à 4 clitiques sont rarement réalisées par le nombre correspondant de syllabes. Souvent, les séquences clitiques apparaissent réduites à une syllabe: [3mə] pour je me et [3lə], [3əl] ou [3la] pour je le/la. Parfois, les clitiques ne forment même plus une syllabe mais juste une attaque consonantique complexe: [3m] pour je me, $[\mathrm{st}]$ pour je te etc. Comme prédit, la tendance générale que nous observons est donc la réduction des séquences clitiques. Celle-ci

145 L'exemple 1637: je vous ne demande pas de réponse à la première question produit par un locuteur non-natif a été exclu, car l'ordre des proclitiques est ici agrammatical en français. 
comprend l'omission de ne, mais également la réduction d'autres clitiques, qui, à son tour, empêche la réinsertion de ne: nous allons montrer, par la suite, qu'il semble impossible d'introduire la particule ne ou le segment [n] dans certaines séquences proclitiques réalisées sous une telle forme réduite ${ }^{146}$.

Deux types de réduction des structures clitiques sont fréquemment observés: la réduction à un seul segment (C ou glide, 7 occurrences) et celle à deux consonnes (6 occurrences).

Le premier type concerne le cas très fréquent de la réduction, voire fusion de la séquence présentative il y a à une seule syllabe [ja]. Le clitique $i l$ est élidé ou bien $i l$ et $y$ sont inséparablement fusionnés jusqu'au point de ne plus pouvoir insérer $n e$.

(219) Type $1:$ la séquence clitique réduite à un segment y a pas de différence [ja.pa.də.di.fe.кãs] (0295

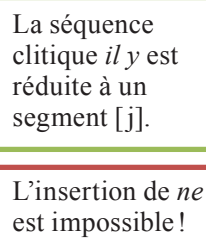
est impossible!

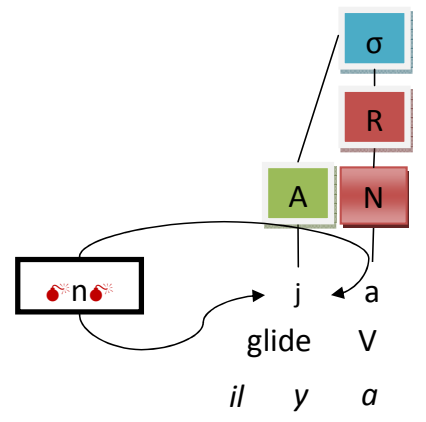

$\mathrm{Si}$, dans des cas d'hypercorrection, ne est inséré dans la séquence il $y$ a, ceci provoque un changement de la structure syllabique, comme le montre l'exemple en (220). Au lieu de prononcer la syllabe simple et universellement préférée [ja], l'insertion de ne obligerait le locuteur

146 Évidemment, comme l'omission de ne fait partie des processus de réduction des séquences proclitiques, l'on pourrait argumenter qu'elle précède la réduction des autres clitiques. Néanmoins, si nous partons de l'idée que les variantes monosegmentales des proclitiques comme [3], [S], [s] s'emploient indépendamment de la variable $\pm n e$, leur présence pourrait tout à fait avoir une influence sur la variable, comme nous le soutenons ici. 
à produire deux syllabes, dont la première serait nue et la deuxième aurait une attaque complexe:

(220) il n'y a pas beaucoup [il.nja.pa.bo.ku] (0124) (= structure syllabique normative)

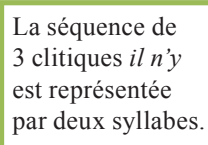

La séquence de 3 clitiques il n'y est représentée par deux syllabes.

L'insertion de $n e$ provoque la production d'une séquence syllabique plus complexe.

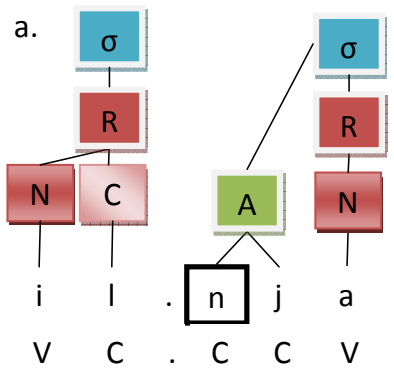

Le fait que cette stratégie complexe ne se trouve que dans les exemples provenant du sous-corpus de distance suggère qu'elle est choisie uniquement et délibérément dans les cas d'hypercorrection, par exemple pendant les examens oraux.

Un deuxième cas de réduction clitique extrême est cité en (221).

(221) Type 2: la séquence clitique en CC

je l'aime pas du tout [3lem.pa.dy.tu] (1660)

\begin{tabular}{|c|}
\hline $\begin{array}{l}\text { La séquence de } \\
\text { deux clitiques je } \\
l(e) \text { est réduite à } \\
\text { deux consonnes. }\end{array}$ \\
\hline $\begin{array}{l}\text { L'insertion de } n e \\
\text { est impossible, } \\
\text { car les attaques } \\
\text { complexes du type } \\
{\left[*^{*} \text { nl] ne sont pas }\right.} \\
\text { admises. }\end{array}$ \\
\hline
\end{tabular}

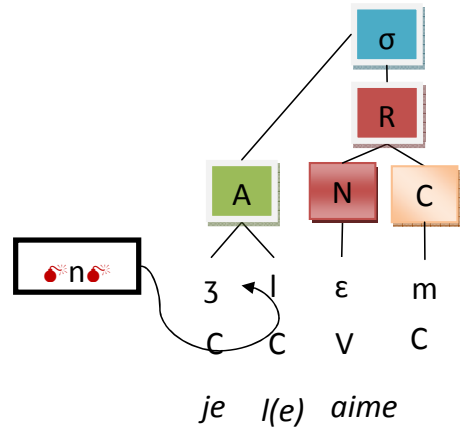


Dans cette structure, les clitiques je et $l(e)$ sont réduits à deux consonnes : [3] et [1]. Ceci veut dire qu'ils ne représentent pas de syllabes indépendantes, mais forment une attaque complexe dans une syllabe CCVC avec la forme verbale aime. Dans une telle syllabe, l'introduction de [n] entre [3] et [1] est impossible, car les attaques triconsonantiques comme *[3nl] ne sont pas admises en français.

D’une manière générale, et comme nous l'avons déjà vu dans la section précédente, nous observons que les séquences proclitiques complexes qui comprennent la variable $\pm n e$ tendent à être réduites. Dans certains cas, cette réduction n'implique que l'omission du ne, mais dans beaucoup d'autres, les clitiques restants sont également réduits, voire fusionnées ou élidés par l'élision du schwa ou de la consonne [1].

Ces réductions érodent le statut de syllabes indépendantes des clitiques et rendent la réinsertion de ne impossible au niveau phonotactique (comme l'observe aussi Larrivée 2014). La réalisation de ne dans les séquences proclitiques reste possible, mais seulement dans des rares cas d'hypercorrection, qui présupposent l'articulation de séquences syllabiques plus longues, contenant les variantes standard des autres clitiques (par exemple [sə] au lieu de [s]). Celles-ci sont défavorisées par le principe eurythmique ( $c f$. Dell 1984, Di Cristo 1998: 197), car elles impliquent la suite de plusieurs éléments atones. D’ailleurs, nous ne trouvons ces exemples que lors des examens oraux, ce qui indique fortement qu'il s'agit de séquences produites délibérément et intentionnellement dans cette situation précise et non pas dans les conversations. Par conséquent, même s'il n'y a aucune incompatibilité générale entre ne et d'autres clitiques, la réalisation de ne semble phontactiquement impossible avec certaines variantes clitiques et peut être qualifiée comme hypercorrection dans les rares séquences proclitiques où elle apparaît.

\subsubsection{L'influence prosodique des sujets lourds}

Nous avons vu dans les sections précédentes que la présence d'un ou de plusieurs proclitiques conduit à des stratégies de compensation prosodique, comme la réduction, voire l'élision des clitiques, ce qui permet de réaliser l'accent initial sur la deuxième syllabe de l'AP. 
Par contre, si un élément lourd et donc accentogène se trouve dans la position de sujet d'un énoncé négatif, aucune compensation prosodique n'est nécessaire.

Si le sujet lourd est court (par exemple nous, qui, Paul etc.), il peut s'unir aux éléments suivants pour former un AP plus large. Cependant, s'il s'agit d'un DP long (par exemple une narratrice, le glossaire de Reichenau), il est souvent réalisé comme un ou plusieurs APs indépendants. Dans les deux cas, Hi peut être réalisé sur un élément lexical au début de l'AP, et la particule ne, se trouvant dans une position inaccentuée, n'est donc pas menacée. Les exemples en (222), dans lesquels la particule ne est toujours réalisée, comportent sans exception un sujet accentogène.

(222) Les sujets accentogènes comme lieux de Hi dans les négatives

a. la mère / ne sait pas / qu'elle s'est séparée (0796)

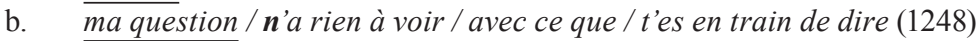

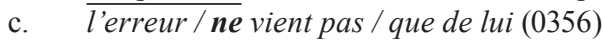

d. ce qui / n'explique pas / pourquoi on parlait grec / dans ces régions-là (2620)

e. qu'aucun savant / philosophe / ni poète / n'a jamais / imaginés / surtout sous les singes (0090)

f. $\quad$ une narratrice / qui ne sert / à rien (0978)

g. et Ulysse / qui ne l'écoute / même pas (0076)

Le schéma en (223) montre les possibles contours prosodiques des énoncés en (222), toujours basés sur la structure de base assumée par Jun/Fougeron (2000) (cf. section 5.2.3). Notons que les possibilités de prononciation des exemples cités en (223) sont multiples et que l'interprétation exacte du contour intonatif de base / $\mathrm{LHiLH}^{*} /$ adoptée par le locuteur reste imprévisible ( $c f$. Jun/Fougeron 2000: 216).

Ce que nous voudrions montrer, à l'aide de la reprise des exemples cités en (222) dans le tableau (223), c'est que, même s'il y a une certaine liberté intonative à l'intérieur d'un AP, les propriétés prosodiques des éléments accentogènes et atones sont toujours à respecter. Autrement dit, les proclitiques (pronoms ou articles) n'apparaissent jamais dans l'une des positions accentuées ( $\mathrm{Hi}$ ou $\mathrm{H}^{*}$ ), car celles-ci sont réservées uniquement aux éléments lourds. 
(223) La position de ne avec les sujets lourds: quelques contours prosodiques exemplaires

\begin{tabular}{|c|c|c|c|c|c|c|c|c|c|c|c|}
\hline \multicolumn{4}{|c|}{$\mathrm{AP}_{1}$} & \multicolumn{4}{|l|}{$\mathrm{AP}_{2}$} & \multicolumn{4}{|l|}{$\mathrm{AP}_{3}$} \\
\hline $\mathrm{L}$ & $\mathrm{Hi}$ & $\mathrm{L}$ & $\mathrm{H}^{*}$ & $\mathrm{~L}$ & $\mathrm{Hi}$ & $\mathrm{L}$ & $\mathrm{H}^{*}$ & $\mathrm{~L}$ & $\mathrm{Hi}$ & $\mathrm{L}$ & $\mathrm{H}^{*}$ \\
\hline & & la & mère & ne & sait & & pas & & & & \\
\hline$m a$ & & ques- & tion & $n^{\prime} a$ & rien & $\grave{a}$ & voir & & & & \\
\hline & & l'err- & -eur & ne & vient & & pas & & que & $d e$ & lui \\
\hline & & ce & $q u i$ & $n^{\prime} e x$ & plique & & pas & & & & \\
\hline & $p h i$ & lo- & sophe & $n i$ & po- & & ète & $n^{\prime} a$ & $j a-$ & & mais \\
\hline une & na & ra & trice & & $q u i$ & ne & sert & $\mathrm{a}$ & & & rien \\
\hline & et & $U_{-}$ & -lysse & & $q u i$ & ne l'é- & coute & & & & \\
\hline
\end{tabular}

Considérons maintenant les contours prosodiques exemplaires des données en (222), illustrés en (223): dans la plupart des cas, les sujets lourds peuvent former un AP indépendant, $c f$. (223)a-d. Dans les exemples (223) a, b et c, il s'agit des DP lexicaux suivants: la mère, ma question, l'erreur. Dans la construction relative libre (cf. Jones ${ }^{3} 2007$ : 513) en (223)d, par contre, l'AP indépendant est constitué par le clitique démonstratif $c e$ (forcément atone) et le pronom relatif accentogène qui. Dans tous ces cas, la particule ne apparaît inévitablement dans l'attaque de l'AP $P_{2}$ qui suit le sujet lourd, où elle occupe une position inaccentuée adjacente à un élément accentogène qui peut porter Hi.

S'il s'agit d'un sujet lourd très long, comme le DP négatif aucun savant / philosophe / ni poète dans l'exemple (223)e, le sujet est 'coupé' en plusieurs APs, mais l'effet reste le même: ne apparaît dans l'attaque du groupe accentuel suivant (ici de l'AP $\mathrm{P}_{3}$ ), qui est une position atone et donc 'protégée'.

Les sujets accentogènes peuvent également former un AP complexe avec des éléments adjacents. Ceci est le cas dans les deux constructions relatives en (223)f et $\mathrm{g}$ : dans ces deux exemples, les antécédents du pronom relatif qui, c'est-à-dire le syntagme lexical une narratrice et le nom propre Ulysse (ici combiné avec la conjonction de coordination et), forment des AP indépendants. Le sujet de la relative, à savoir le pronom accentogène qui, est combiné avec le matériel suivant pour former un AP. Comme qui est le seul élément lourd dans la périphérie gauche de 
la relative, il est forcément le seul à pouvoir occuper la position $\mathrm{Hi}$, tandis que la particule atone ne occupe (de nouveau) une position sans accent (L) à l'intérieur de l'AP.

Dans tous les cas discutés, le matériel morphologique est donc distribué sur les APs selon ses propriétés phonologiques, de manière à ce que les éléments atones comme ne puissent apparaître dans l'une des positions inaccentuées.

\subsubsection{L’influence prosodique des sujets redoublés}

Le facteur des sujets redoublés est rarement pris en compte dans les explications théoriques de la variation \pm ne, mais l'approche diglossique (cf. Culbertson 2010, Culbertson/Legendre 2008, Massot 2010, Zribi-Hertz 2011 et section 2.3.3) constitue une exception à cette généralisation: Culbertson (2010), Culbertson/Legendre (2008) et Massot (2010) soutiennent que, dans les constructions redoublées, les clitiques du français de l'immédiat sont des marques d'accord préfixées qui excluent ne.

L'analyse de corpus a montré qu'il n'y a effectivement aucune réalisation ne avec les sujets redoublés ( $c f$. section 4.4.1.3). En même temps, l'analyse a fait ressortir que les éléments pronominaux redoublés sont presque exclusivement des clitiques légers et que, parmi ceux-ci, les variantes monosegmentales, qui excluent catégoriquement la présence de $n e$, sont surreprésentées. Il semble donc convainquant d'assumer que l'absence de ne dans les structures redoublées n'est ni une coïncidence, ni une preuve pour le statut affixal des proclitiques, comme le soutiennent Culbertson (2010), Culbertson/Legendre (2008) et Massot (2010), mais qu'elle est liée d'une manière ou d'une autre aux propriétés syllabiques des éléments employés dans les structures redoublées.

Ces éléments sont généralement des XP lourds suivis par des clitiques légers, comme l'illustrent les exemples sous (224).

(224) La structure prosodique des sujets redoublés

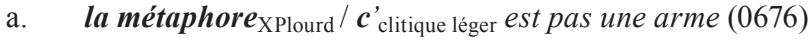

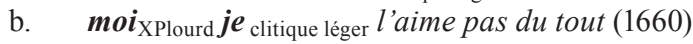


Considérons, par la suite, l'effet des sujets redoublés selon l'hypothèse prosodique. Ceci signifie que nous examinons les contours prosodiques typiques de ces structures et les possibilités d'y placer Hi, comme nous l'avons fait pour les sujets simples dans les sections précédentes.

(225) L'incompatibilité prosodique entre ne et les sujets redoublés

a.

\begin{tabular}{|c|c|c|c|c|c|c|c|}
\hline \multicolumn{4}{|c|}{$\mathrm{AP}_{1}$} & \multicolumn{4}{|l|}{$\mathrm{AP}_{2}$} \\
\hline $\mathrm{L}$ & $\mathrm{Hi}$ & $\mathrm{L}$ & $\mathrm{H}^{*}$ & $\mathrm{~L}$ & $\mathrm{Hi}$ & $\mathrm{L}$ & $\mathrm{H}^{*}$ \\
\hline la & $m e ́-$ & $t a-$ & phore & $\begin{array}{c}c \text { 'est } \\
c e{ }^{\prime \prime n} \text { 'est }\end{array}$ & pas & une & arme \\
\hline & $m o i$ & $\begin{array}{c}\text { je } \\
\text { je } \stackrel{\text { n }}{ } \text { ne }\end{array}$ & l'aime & & pas & $d u$ & tout \\
\hline
\end{tabular}

L'exemple (224)a, dont le contour prosodique figure en (225)a, est un cas exemplaire de redoublement du sujet dans notre corpus ( $c f$. section 4.4.1.3), c'est-à-dire que le contour intonatif illustré en (225)a vaut aussi pour de nombreux autres exemples qui ont la même structure morphosyntaxique.

Généralement, l'élément lourd dans un sujet redoublé constitue un AP indépendant, comme par exemple le DP polysyllabique la métaphore en (225)a. L'élément pronominal coréférentiel suit normalement dans la position initiale de l'AP suivant, qui est inaccentuée.

Dans l'exemple (225)a, comme dans la majorité de ces structures, il s'agit de la variante monosegmentale [s] du démonstratif ce ( $c f$. diagramme (144)b dans la section 4.4.1.3) suivi par la forme verbale est. Nous avons vu lors de l'analyse de corpus que, dans les séquences presque lexicalisées c'est $+N E G$, ne est quasiment exclu ( $c f$. section 4.4.1.3). L'introduction de ne dans ce type de séquence signifierait que le clitique ce devrait être réalisé sous sa forme standard [sə], une tournure phonotactiquement défavorisée qui est choisie uniquement en tant qu'hypercorrectisme ( $c f$. section 5.2.4.2). Par conséquent, vu que dans l'ensemble du corpus environ $40 \%$ des séquences à sujet redoublé ont la forme XPlourd + c'est $(+\mathrm{NEG})$, l'absence de $n e$ dans ces $40 \%$ des cas s'explique par son caractère quasi lexicalisé dans ces séquences. 
L'exemple (225)b illustre un autre cas très typique de sujet redoublé. Il s'agit d'un pronom lourd, dans ce cas moi, qui est repris par un clitique léger, ici je. Les pronoms lourds peuvent, comme les XPs lourds, former un AP indépendant, mais dans de nombreux cas ils sont intégrés avec le matériel qui suit dans un AP plus long. Le contour intonatif d'une telle structure est illustré par l'exemple (225)b: l'élément accentogène moi apparaît dans la position $\mathrm{Hi}$, tandis que le clitique léger le suit dans la position inaccentuée. L'introduction de ne dans cette séquence conduirait à la présence de deux éléments atones consécutifs, défavorisée par le principe eurythmique (cf. Dell 1984, Di Cristo 1998: 197).

Dans les structures à sujet redoublé, et indépendamment du fait que l'élément lourd soit un DP, un nom propre ou un pronom lourd et qu'il forme un AP indépendant ou non, le clitique léger s'emploie toujours dans une position inaccentuée. La réalisation de ne conduirait donc toujours à une structure prosodique fortement défavorisée, qui est évitée par la chute de ne. Il ne s'agit donc pas d'une incompatibilité syntaxique mais prosodique entre ne et les sujets redoublés. Nous ajoutons que la structure XPlourd $+c^{\prime} e s t+N E G$, dans laquelle l'absence de ne semble déjà presque lexicalisée, est surreprésentée parmi les constructions à sujet redoublé (cf. section 4.4.1.3) et que l'approche prosodique des sujets redoublés nous permet d'expliquer un détail observé par Sturm (1981 : 134-135): la combinaison d'un sujet DP avec un clitique objet, donc d'un élément lourd suivi par un élément léger, conduit à l'omission de $n e . \mathrm{Vu}$ que les structures $\mathrm{DP}_{\text {lourd }}+$ clitique $_{\text {léger }} \pm n e$ ont toujours le même contour prosodique (indépendamment de la fonction grammaticale du clitique), les cas observés par Sturm (1981 : 134-135) sont analysables comme les sujets redoublés selon le schéma prosodique illustré en (225).

\subsubsection{L'influence prosodique du type de phrase}

Les analyses de corpus ont montré que, par rapport aux principales, la réalisation de ne est significativement plus fréquente dans les constructions subordonnées et en particulier dans les relatives ( $c f$. sections 2.2.5.6 et 4.4.5.1). Même si l'effet du type de phrase sur \pm ne est moins 
fort que celui des sujets, les subordonnées semblent quand même posséder, tout comme les sujets lourds, une particularité qui 'protège' la particule de négation de l'omission.

Au niveau syntaxique et prosodique, les subordonnées manifestent sans exception un élément initial que les principales ne possèdent pas : la conjonction de subordination. Fidèlement à l'approche prosodique proposée ici, nous soutenons que c'est cet élément qui peut porter l'accent initial et qui facilite donc la réalisation de ne. En (226) et (227) figurent quelques exemples du corpus qui soutiennent cette hypothèse.

(226) Les subordonnées négatives: quelques exemples

a. de mettre quelqu'un / qui n'est pas dévot (2114)

b. une narratrice qui ne sert à rien (0978)

c. ce qui n'explique pas / pourquoi on parlait grec/dans ces régions-là (2620)

(227) La position de ne dans les subordonnées négatives: quelques contours prosodiques

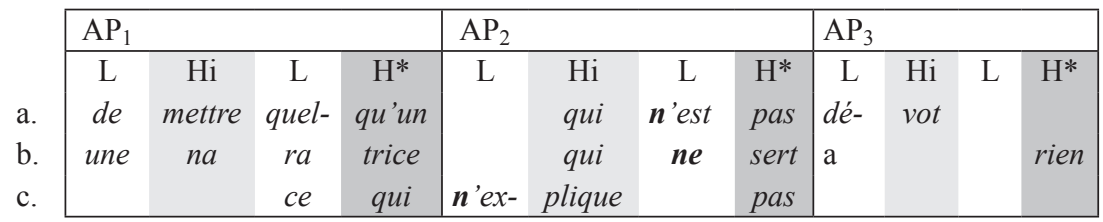

La présence d'une conjonction de subordination dans une phrase signifie qu'il y a, par rapport aux principales, une syllabe ultérieure à intégrer dans la structure intonative de l'énoncé. Contrairement aux sujets accentogènes, les éléments subordonnants les plus fréquents, comme le pronom relatif qui, sont trop courts pour former un AP indépendant (ceci ne vaut pas pour les subordonnants plus rares comme tandis que, parce que etc.). Toutefois, la présence de qui donne lieu, en s'ajoutant au matériel adjacent, à des APs assez complexes, fait qui s'observe très bien dans les relatives qui figurent en (227)a et b.

Les antécédents lourds, dans nos exemples il s'agit du pronom indéfini quelqu'un et du NP narratrice, forment le premier AP. Ainsi, le pronom relatif accentogène qui apparait dans la position initiale du deuxième $\mathrm{AP}$ : vu que qui peut porter $\mathrm{Hi}$, la particule ne peut être intégrée en position inaccentuée dans le deuxième AP. 
De plus, comme le pronom qui coïncide avec le sujet, il y a donc toujours un sujet accentogène dans ces constructions ( $c f$. section 5.2.4.3). Nous notons également que qui se termine par une voyelle, ce qui signifie que les possibilités pour ne d'apparaître en position intervocalique sont élevées. Comme nous l'avons vu dans l'analyse de corpus, les séquences $\mathrm{V}+n e \mathrm{~V}$ sont fortement préférées $(60 \%+n e)$ aux séquences $\mathrm{V}-n e \mathrm{~V}$ ( $c f$. section 4.4.6) et ceci s'observe dans l'exemple (227)a, qui comprend la séquence qui n'est, dans laquelle la présence de [n] empêche le hiatus dans qui est. Finalement, dans l'exemple (227) c, figure une construction relative libre: ici, les deux éléments qui introduisent la construction, à savoir le clitique ce et le pronom qui, forment un AP indépendant, suivi par ne, qui apparaît de nouveau dans la position initiale inaccentuée du deuxième AP.

Nous avons donc montré que, similairement aux sujets lourds, la présence d'un élément subordonnant, surtout celle du pronom relatif $q u i$, dans une phrase modifie la structure prosodique de telle sorte que la présence de ne est favorisée. Comme l'élément initial de la subordonnée peut porter Hi, la particule ne peut apparaître dans une position inaccentuée à l'intérieur de l'AP. Il semble également convainquant de supposer que l'effet des subordonnées sur \pm ne soit moins fort que celui des sujets lourds, parce que les subordonnants très fréquents comme que et qui sont prosodiquement moins proéminents que la majorité des DP lexicaux.

\subsubsection{L'applicabilité de l'approche prosodique à d'autres contextes négatifs}

Dans ce qui suit, nous discutons brièvement si les constructions impératives, les infinitives, les gérondifs et les participes présents négatifs, qui sont rares voire inexistants dans notre corpus, seraient en principe également traitables selon l'approche développée ici.

Avec les participes présents et les gérondifs, la variable $\pm n e$ apparaît dans la même position préverbale que dans les constructions finies, comme les exemples en (228) le montrent: 
(228) Le gérondif et le participe présent négatifs

a. les chasseurs [/] ne sachant pas [/] chasser condamnés ${ }^{147}$

b. quand je me réveille comme ça // et mes premiers gestes /c'est d'aller retourner les fromages / parce que je suis capable de faire ça / en (n')étant pas réveillée // (C-ORAL-ROM)

Si ces constructions sont à traiter de la même façon que les constructions finies, nous soutenons que, à cause de l'absence du sujet, les taux de réalisation de ne avec les participes présents devraient être relativement élevés, car ne tend à occuper la position initiale inaccentuée d'un groupe accentuel (AP), comme dans l'exemple (228)a.

Avec les gérondifs, la situation est légèrement différente, car la présence obligatoire du clitique en altère la structure prosodique en déplaçant ne dans la deuxième position, qui est souvent accentuée et donc défavorable à sa réalisation: en \pm ne sachant pas. Selon l'approche prosodique, nous nous attendrions donc à une présence plus faible de ne avec les gérondifs qu'avec les participes présents négatifs.

Malheureusement, au niveau empirique, ces constructions semblent tellement rares dans les corpus oraux qu'une vérification quantitative de ces hypothèses est impossible. Les participes présents niés, illustrés à l'aide de l'exemple (228)a, extrait d'un journal, semblent introuvables dans les corpus oraux et le gérondif négatif cité en (228)b est le seul résultat d'une recherche de la séquence $<$ ant pas $>$ dans le corpus C-ORAL-ROM ( $c f$. Cresti/Moneglia 2005). Finalement, avec les gérondifs négatifs, la présence ou absence de ne est souvent imperceptible à cause de la liaison obligatoire, ce qui constitue une difficulté méthodologique ultérieure, comme l'on peut le voir dans l'exemple (228)b. Nous devons donc laisser la question de $\pm n e$ avec les gérondifs et les participes présents aux futurs travaux de recherche.

Les infinitifs négatifs, par contre, sont plus nombreux dans les corpus, comme les exemples en (229) le montrent, et nous y observons aussi bien l'absence que la présence de ne:

147 <http://www.liberation.fr/societe/2013/01/21/les-chasseurs-ne-sachant-paschasser-condamnes_875685>, consulté le 19 mars 2013. 
(229) L'infinitif négatif (exemples de C-ORAL-ROM)

a. je garderai toute ma vie / une espèce de [/] de frustration au fond de moi / celle de ne pas avoir été doté par dame nature

b. puis je pense pas avoir [/] franchement la tête euh d'une personne qui est triste ou non

c. c'est surtout quelqu'un qui a besoin d'être aimé / je veux dire qui supporte pas de [/] de pas être au centre des conversations [/] de pas être au centre des trucs

d. je trouve dommage de ne pas [/] de ne pas s'exprimer

e. un dictionnaire de langue / pourrait-il ne pas être un ouvrage linguistique?

f. c'est \# d'apprendre / \# un certain nombre d'automatismes qui permettent par exemple de ne pas manipuler une arme

g. pour ne pas dire que [/] qu'ils sont obèses

h. ils dorment en bas // pour pas euh salir en haut

i. $\quad$ c'est-à-dire que pour pas éteindre vraiment la vie minière / on a fait beaucoup d'associations

Contrairement aux constructions finies, dans les infinitifs, la variable $\pm n e$ précède la particule négative pas, qui est suivie, elle, de l'infinitif du verbe: \pm ne pas VINF (sauf avec les auxiliaires et certains verbes modaux qui peuvent également apparaître entre \pm ne et pas, cf. Marie semble n'avoir pas aimé ce film, Jones ${ }^{3} 2007$ : 349).

D'après l'approche prosodique, nous devrions donc nous attendre à une présence élevée de ne avec les infinitifs par rapport aux constructions finies, car l'absence du sujet place souvent la variable $\pm n e$ dans la position initiale d'un AP, où elle est 'protégée' prosodiquement. En effet, dans le corpus d'Ashby (1981: 678), l'absence de ne avec les infinitifs $(64 \%+n e)$ est clairement plus élevée qu'avec les constructions finies indépendantes $(30 \%+n e)$.

Comme nous l'avons déjà montré dans la section 5.2.4.5, les caractéristiques prosodiques des subordonnées, y compris les constructions à l'infinitif (qui comprennent souvent la préposition accentogène pour en position initiale), diffèrent prosodiquement de celles des principales. Il semble donc en principe possible d'étendre également l'approche prosodique aux infinitifs négatifs.

Considérons finalement les constructions impératives négatives, illustrées par les exemples en (230) et (231). 
(230) Les constructions impératives négatives (exemples de T-zéro/C-ORAL$\mathrm{ROM})$

a. alors parlez pas (1568)

b. ne repassez pas jamais $(2590)^{148}$

c. fais pas cette tête (1090)

d. je vais vous sortir une documentation maintenant // alors bougez pas

e. j'ai dit non [/] non / ne venez pas à Aix / ça va me perturber parce que / n'oubliez pas / il proclame une parole / il n'y aura ni pluie / ni rosée

f. non c'est mon fond de culotte qui se déchire // ne riez pas / cette chaise est épouvantable

(231) Impératives négatives avec d'autres proclitiques (exemples de C-ORAL$\mathrm{ROM})$

a. alors il lui dit ne t'in [/] t'inquiète pas / j'ai préparé un endroit où tu vas aller

b. mon monsieur_Pl m'avait dit / t'inquiète pas / en grammaire tu es prêt

Les deux variantes, avec et sans ne, peut être observées dans les corpus T-zéro et C-ORAL-ROM. Si des clitiques sont présents, comme dans les exemples sous (231) (ici il s'agit du clitique réfléchi $t(e)$ ), nous les trouvons généralement en position préverbale comme dans les indicatifs.

Par conséquent, l'approche prosodique pourrait être appliquée à la variation \pm ne dans les constructions impératives illustrées dans les exemples en (230) et (231). L'hypothèse pour ce type de construction serait que l'absence de ne est plus fréquente si d'autres proclitiques s'ajoutent à la variable $\pm n e$, car nous avons vu que la présence d'un seul proclitique est prosodiquement favorisée, tandis que plusieurs proclitiques peuvent s'exclure mutuellement. Cependant, faute de données pertinentes dans notre corpus, cette hypothèse ne sera pas testée empiriquement dans le cadre du présent travail. Nous laissons donc une autre piste pour des futurs travaux de recherche.

On trouve également des tournures impératives négatives sans $n e$, mais avec un pronom enclitique (cf. Rowlett 2014).

148 La discussion de cet exemple dans la section 5.1.4 montre qu'ici la présence de $n e$ est avant tout explicable en fonction du contexte pragmatique. 
(232) Les constructions impératives négatives sans ne avec l'enclise du pronom (exemples provenant de Google)

a. Ah non, touche-moi pas! Tu me salis ! ${ }^{149}$

b. Vas-y pas Gaston! ${ }^{150}$

c. Prends-le pas mal! ${ }^{151}$

d. Parles-en pas! $!^{152}$

(233) ne est impossible dans les impératives négatives avec enclise pronominale

a. Ah non, (*ne) touche-moi pas! Tu me salis!

b. (*ne) vas-y pas Gaston!

c. (*ne) prends-le pas mal!

d. (*ne) parles-en pas!

Dans ce type de construction, la présence de ne est catégoriquement exclue, comme les données en (233) le montrent, et. l'insertion de ne semble en quelque sorte bloquée par l'enclise pronominale.

L'insertion de la variable $\pm n e$ n'est possible que si les clitiques sont placés avant le verbe:

(234) \pm ne est possible dans les impératives négatives avec proclise pronominale

a. Ah non, \pm ne me touche pas! Tu me salis!

b. \pm n'y vas pas Gaston!

c. $\quad \pm$ ne le prends pas mal!

d. \pm n'en parles pas!

Étant donné que ne est catégoriquement exclu dans les impératives négatives avec une enclise, comme en (233), nous constatons qu'il n'y a aucune variation \pm ne dans cette construction particulière. L'approche prosodique, envisageant la variation linguistique, semble donc, au premier abord, moins apte à expliquer cette restriction qu'une approche

149 Cet exemple a été prononcé en France par un homme refusant la poignée de main de Nicolas Sarkozy lors du Salon de l'Agriculture en 2008 (cf. < http://fr. wikipedia.org/wiki/Casse-toi,_pauv\%27_con_> consulté le 18 avril 2013).

150 Un vers de la chanson Le lion de Jacques Brel (cf. <http://www.frmusique.ru/ texts/b/brel_jacques/lion.htm> consulté le 18 avril 2013).

151 Un vers de la chanson Le pyromane de Karkwa, un groupe du Québec $(c f .<$ http:// offqc.com/2011/09/23/prends-le-pas-mal/> consulté le 18 avril 2013)

152 Une entrée dans un blog québécois (cf. $<$ http://blogue.infodimanche.com/drouin/ 2012/10/05/\%C2\%AB-parles-en-pas-\%C2\%BB/> consulté le 18 avril 2013). 
syntaxique: il existe, en effet, de nombreuses tentatives d'expliquer syntaxiquement le contraste entre (233) et (234) ainsi que la distribution des clitiques dans les structures impératives en général ( $c f$. les références citées ci-dessous).

En français moderne, trois des quatre possibilités logiquement possibles de placer les clitiques dans les impératives sont attestées:

(235) Pro- et enclise dans les impératives positives et négatives en français moderne

\begin{tabular}{|l|l|l|}
\hline & positif & négatif \\
\hline proclise & $-{ }^{*} y$ vas! & $+n^{\prime} y$ vas pas! (ordre correct en français standard, $c f$. (234)) \\
\hline enclise & $+v a-z-y !$ & $+v a-z-y$ pas! (rare mais attesté, $c f$. (233) \\
\hline
\end{tabular}

Tandis que l'impératif positif est exprimé à l'aide d'une inversion clitique-verbe (va-z-y!), l'ordre inverse (*y vas!) n'étant pas admis, l'impératif négatif permet aussi bien la proclise acceptée selon la norme ( $n$ 'y vas pas!) que l'enclise (va-z-y pas!), même si ce dernier cas est rare et banni par les grammairiens.

Les approches syntaxiques qui essaient d'expliquer les données illustrées en (235) appartiennent généralement à deux groupes d'approches théoriques: d'un côté, les différences sont modelées par des contraintes en termes de la théorie d'optimalité (pour le français et ses variétés $c f$. Hirschbühler/Labelle 2000, 2001, Labelle/Hirschbühler 2001, pour d'autres langues $c f$. Anderson 1996, 2000, Legendre 1996, 1997, 2000); de l'autre, elles sont expliquées par le rôle du déplacement verbal et des effets de blocage (pour le français $c f$. Hirschbühler/ Labelle 2006, Rowlett 2014, pour d'autres langues romanes $c f$. Benincà 1991, 1995, Martins 2000 [1995] et Rivero 1997).

Pour des raisons d'espace, nous ne pouvons pas expliquer ici en détail toutes les approches citées ci-dessus et par conséquent, nous nous limiterons à résumer à titre exemplaire les idées de Rowlett (2014).

En se basant sur une analyse des clitiques selon Shlonsky (2004) et dans le cadre de l'approche générative dite 'cartographique' selon Rizzi (1997), Rowlett (2014) fournit une analyse de l'ordre respectif des clitiques et du verbe dans les constructions impératives positives et né- 
gatives en français contemporain. Rowlett (2014) soutient que l'enclise dans les impératives positives, illustrée en (235), est possible grâce à un mécanisme qui permet la vérification des marques flexionnelles du verbe (comme par exemple de la liaison obligatoire dans vas-y [vazi]) et du mode irréel [IRR], propre au verbe à l'impératif, dans une seule tête fonctionnelle. Ceci donne alors lieu à un déplacement du verbe à gauche des clitiques et génère l'enclise vas-y ( $c f$. l'annexe XIV pour la structure syntaxique). En revanche, dans les impératives négatives correctes selon la norme, la négation intervient entre les projections du mode irréel et de l'information flexionnelle, ce qui bloque la vérification des marques flexionnelles et de [IRR] dans une seule tête. Par conséquent, l'enclise n'est pas possible et ces structures présentent la proclise: n'y vas pas (cf. l'annexe XV pour la structure syntaxique).

La particularité des impératives négatives non-standard contenant une enclise, comme vaz-y pas en (232), s'explique, selon Rowlett (2014), par le fait que, tout comme la négation de constituant, la particule négative n'a dans ces constructions qu'une portée locale ${ }^{153}$ et n'est pas associée à une projection fonctionnelle négative $(\mathrm{NegP})$ qui pourrait contenir le clitique $n e^{154}$. Selon Rowlett (2014), cette évolution corres-

153 Afin d'illustrer la portée négative locale, Rowlett (2014) cite l'exemple suivant: Parles'en pas juste a tes ami(e)s mes aussi a tes parents! [sic] (cf. <http:// www.vrak.tv/missvrak/courrier-du-coeur/je-me-fais-intimider-4278/>, consulté le 7 février 2013).

154 Rowlett (2014) propose une deuxième analyse pour les occurrences des constructions du type vaz-y pas. Selon lui, ces ocurrences pourraient être interprétées comme des manifestions d'une autre grammaire du français (éventuellement comparable à la grammaire 1, retenue par l'approche diglossique et que nous décrivons dans la section 2.3.3 et rejetons en section 5.1.3). Dans une telle grammaire, la négation n'est pas associée à une projection fonctionnelle et ne bloque donc pas la vérification commune des marques flexionnelles et modales.

Par ailleurs, un critique anonyme de Rowlett (2014) propose une troisième solution syntaxique, selon laquelle l'absence du morphème négatif ne dans la tête de NegP permet la vérification de la marque négative avec les marques flexionnelles et modales à l'intérieur d'une seule tête fonctionnelle. Ceci génère alors l'ordre des mots de l'impérative positive vaz-y ( $c f$. annexe XIV), suivi par la particule négative pas dans une position plus basse, et donne lieu à la structure correcte: vaz-y pas. 
pondrait à une innovation naturelle et prévisible en termes du cycle de Jespersen $(1917,1924)$.

Nous avons vu que, comme il n'y a pas de variation $\pm n e$ dans les impératives négatives avec enclise pronominale, mais plutôt des restrictions de type syntaxique, les approches syntaxiques, comme celle de Rowlett (2014), offrent des perspectives convenables pour motiver le placement des clitiques et le blocage de $n e$ avec les enclitiques illustrés en (235). Nous admettons donc que la prosodie ne peut rien apporter à ce problème d'un point de vue synchronique, car en français moderne les restrictions sont désormais de type syntaxique.

Cependant, l'approche prosodique n'est pas complètement inutile pour comprendre la distribution des clitiques en français moderne, illustrée en (235), car si nous comparons les données du français contemporain avec celles de l'ancien français et du français classique, nous découvrons que la distribution actuelle des clitiques remonte probablement à une règle syntactico-prosodique, à savoir la loi Tobler/Mussafia (cf. Mussafia 1886, Tobler 1912 [1875]).

Comme en français moderne, Hirschbühler/Labelle (2006) trouvent déjà au début du $17^{\mathrm{e}}$ siècle l'enclise pronominale sans ne dans les constructions impératives négatives, à savoir dans la production langagière du futur roi Louis XIII transcrite par son médecin Jean Héroard ( $c f$. Ernst 1985 et section 2.2.1.1):

(236) Les impératives négatives sans ne avec enclise pronominale dans le journal d'Héroard (cf. Hirschbühler/Labelle 2006: 202)

Madame esternue, chacun luy dit «Dieu vous soit en aide». Il s'en fasche: "Non, dite luy pa Dieu vous soit en aide» (3; 02. Nov. 1604, p. 548)

'Madame sneezes, everyone says to her "God bless you". He gets angry: "No don't tell her God bless you""

Apparemment, les exemples correspondants en français moderne en (232) ne sont donc pas une invention récente, mais remontent déjà (tout comme l'absence de ne dans les interrogatives inverties, $c f$. section 2.1.2.1) au français classique.

À la différence du français moderne, à l'époque du jeune Louis XIII, les quatre possibilités de placement des clitiques dans les impératives positives et négatives sont attestées ( $c f$. Hirschbühler/Labelle 2006): 
(237) Proclise et enclise dans les impératives positives et négatives dans le journal d'Héroard ( $c f$. Hirschbühler/Labelle 2006)

\begin{tabular}{|l|l|l|}
\hline & positif & négatif \\
\hline proclise & $+y$ vas! $(c f .(238))$ & + n'y vas pas! $(c f .(241))$ \\
\hline enclise & $+v a-z-y !(c f .(239))$ & $+v a-z-y$ pas! $(c f .(236))$ \\
\hline
\end{tabular}

Hirschbühler/Labelle (2006: 189) expliquent la coprésence des quatre variantes dans le corpus d'Héroard à l'aide de l'existence de deux grammaires en concurrence selon Kroch (2000) et Lightfoot (1999).

Ils soutiennent qu'en ancien français, qui est en voie de disparition à l'époque du journal d'Héroard, le placement des clitiques était strictement réglé par la loi Tobler/Mussafia ( $c f$. Hirschbühler/Labelle 2006: 190). Selon la loi Tobler/Mussafia, les clitiques (objet) apparaissent généralement en position préverbale, $c f$. (238), sauf si celle-ci est la position initiale d'un énoncé, $c f$. (239). ${ }^{155}$

(238) La proclise selon Tobler/Mussafia en ancien français

(cf. Hirschbühler/Labelle 2006: 190)

a. Un vaissel nuvel me portéz é sél m'i metéz

'Bring me a new dish and put salt in it for me' (QLR: 176,20 in de Kok: 78)

b. Sire, (...) ensi vous avint il?

'Sire, (...) did it happen to you so?' (Merlin II 46 in Skårup: 161)

(239) L'enclise selon Tobler/Mussafia en ancien français

(cf. Hirschbühler/Labelle 2006: 190)

a. Pursiu les, senz dute les prendras, sis ociras.

'Chase them, without doubt you will catch them, and kill them'

(QLR: 58,8 in Kok: 84)

155 La loi Tobler/Mussafia distingue plusieurs étapes ( $c f$. Hirschbühler/Labelle 2000: 165). Dans la première phase, à laquelle nous faisons référence ici, les clitiques étaient exclus de la position initiale dans tout type de phrase. Dans la deuxième étape, ils sont admis en position initiale si la phrase est introduite par une conjonction de coordination comme et. Dans les étapes successives, les clitiques sont admis en position initiale dans toutes les phrases, d'abord à l'exception des volitives (c'est-à-dire des impératives et des hortatives) (étape 3), puis seulement à l'exception des impératives (étape 4), jusqu'à apparaître toujours en position postverbale dans les impératives positives (étape 5). 
b. Vint s'en al tabernacle

'[She] went to the tabernacle'

c. Conois la tu?

'Do you know her?'

(QLR I,9)

(Queste: 112,17 in de Kok: 82)

Les occurrences d'enclise dans les impératives positives et négatives dans le corpus d'Héroard sont donc expliquées par l'effet de la loi Tobler/Mussafia: sans enclise, les clitiques devraient apparaître en position initiale, ce qui était impossible en ancien français.

Par contre, dans le même corpus, les deux autres variantes de placement des clitiques, à savoir la proclise dans les impératives positives et négatives, sont aussi attestées:

(240) L'enclise et la proclise dans les impératives positives coordonnées dans le corpus Héroard ( $c f$. Hirschbühler/Labelle 2006: 194)

Descendé la pui la remeté.

(3; 09. June 1605 , p. 687)

'Bring her down, then put her back.'

(241) La proclise avec et sans ne dans les impératives négatives dans le corpus Héroard ( $c f$. Hirschbühler/Labelle 2006: 208, 204)

a. Ma soeur, n'en mangé pa, i n'e pa bon. $\quad$ (7; 02. Nov. 1608, p. 1543) 'My sister, don't eat that, it's not good.'

b. Hee maman ga me doné pa le fouet... $\quad$ (4; 03. Dec. 1605, p. 851)

'Hey Maman Ga, don't whip me'

Du fait que les quatre variantes illustrées dans le tableau (237) sont attestées, Hirschbühler/Labelle (2006: 190) concluent que la grammaire de l'ancien français, appliquant la loi Tobler/Mussafia, est en concurrence avec la grammaire surgissante du français moderne, qui ne l'applique plus de manière cohérente. Pour cette raison, la proclise dans les impératives négatives, comme illustrée en (241), qui est la variante privilégiée par la norme en français moderne, est déjà admise à l'époque du jeune Louis XIII.

En français contemporain, les effets de la loi Tobler/Mussafia, comme ceux illustrés en (239)b et c, ont largement disparu. Par contre, l'enclise dans les impératives positives, qui remonte à la loi Tobler/ Mussafia, a été maintenue et codifiée ( $c f$. Hirschbühler/Labelle 2000, 2003). 
Nous pouvons donc supposer, dans la ligne de pensée de Hirschbühler/Labelle (2003), que l'enclise dans les impératives négatives excluant la présence du proclitique $n e$ (cf. (232) et (233)), bannie du français moderne par la norme, mais toujours attestée dans certains cas, provient également de la loi Tobler/Mussafia: pour cette raison, le placement de $n e$ clitique en position initiale reste agrammatical.

Depuis l'ancien français, les structures illustrées en (236), qui ont recours à l'enclise dans les impératives négatives, se sont apparement maintenues dans divers dialectes français ( $c f$. Hirschbühler/Labelle 2003), en français québécois et même en français européen moderne dit 'populaire' ( $c f$. les exemples en (232)). Elles coexistent avec les structures correspondantes beaucoup plus fréquentes qui contiennent une proclise et sont admises par la norme.

Nous n'essayons pas d'expliquer ici le fonctionnement syntaxique de la loi Tobler/Mussafia ou d'autres contraintes clitiques et renvoyons à la littérature abondante consacrée à ce sujet, comme par exemple l'approche de Rowlett (2014) de la distribution des clitiques dans les impératives, esquissée brièvement ci-dessus (cf. aussi Anderson 1996, 2000, Benincà 1991, 1995, Hirschbühler/Labelle 2000, 2001, 2006, Labelle/ Hirschbühler 2001, Legendre 1996, 1997, 2000, Martins 2000 [1995], Rivero 1997 etc.).

Nous pouvons conclure que les structures du type $\left({ }^{*} n e\right)$ va-z-y pas! en français moderne remontent à une règle prosodique obsolète, à savoir la loi Tobler/Mussafia, qui a encore des effets syntaxiques importants dans certaines variétés du français. Par conséquent, nous pouvons affirmer que la prosodie joue effectivement un rôle pour l'explication de l'absence de ne dans les impératives négatives à enclise, du moins d'un point de vue diachronique.

\subsubsection{Conclusion intermédiaire}

Dans les sections ci-dessus, nous avons montré que l'influence des différents types de sujet (légers, lourds et redoublés), des séquences proclitiques et du type de phrase sur la variable \pm ne est explicable dans chaque cas à travers les mêmes principes prosodiques. Ceci vaut aussi, dans une certaine mesure, pour la variable \pm ne dans d'autres contextes 
syntaxiques, comme les participes présents, les gérondifs, les infinitives et les impératives.

En français contemporain, il existe un accent initial secondaire (Hi) en plus de l'accent final principal du groupe rythmique (AP). Étant donné que Hi tend à être réalisé au début d'un AP, les énoncés comprenant plus d'un proclitique (comme par exemple je ne) tendent à être réduits, car les proclitiques sont atones par définition et ne peuvent donc pas porter Hi. Le phénomène qui nous intéresse en particulier ici est la stratégie de réduire un AP par l'omission de ne. De plus, nous avons vu que les autres clitiques sont également souvent réduits, fusionnés ou omis. Les sujets redoublés favorisent eux aussi l'omission de ne, car la position inaccentuée dans l'AP y est généralement occupée par une variante monosegmentale comme [s] ou [3] qui est incompatible avec ne.

Contrairement à cela, les sujets lourds et les subordonnées impliquent habituellement la réalisation de ne. Dans les deux cas, ne apparaît dans une position inaccentuée à l'intérieur d'un AP et est donc 'protégé' prosodiquement. De la même façon, les participes présents, les infinitifs et les impératifs contenant une proclise incitent à la réalisation de la particule négative, car, dans ces constructions, elle apparaît dans la position initiale inaccentuée de l'AP.

Dans l'ensemble, l'approche prosodique permet une explication cohérente de la forte influence des facteurs linguistiques qui s'est manifestée lors notre analyse de corpus ( $c f$. sections 4.4.1, 4.4.5.1 et 4.5) ainsi que dans toutes les autres études relatives à ce sujet ( $c f$. Ashby 1976, 1981, 2001, Armstrong/Smith 2002, Coveney ${ }^{2} 2002$, Hansen/Malderez 2004 dont les résultats sont décrits dans la section 2.2.5.1). Une telle intégration de plusieurs facteurs linguistiques dans une approche explicative de la variation \pm ne est plutôt unique dans son genre ( $c f$. section 5.1): la nature prosodique des sujets grammaticaux dans les énoncés négatifs, par exemple, n'a jamais été intégrée de manière convaincante dans les explications théoriques. Ceci est dû, entre autres, au fait que les types de sujet ont été traités, jusqu'à présent, comme une dichotomie entre les pronoms sujet (clitiques) et les DPs lexicaux. Une telle classification binaire des sujets grammaticaux, adoptée par exemple par Culbertson (2010) et Culbertson/Legendre (2008), qui se basent à leur tour sur les 
travaux de Auger (1994), Miller/Sag (1997), Roberge (1990) et ZribiHertz (1994), ignore le fait qu'à l'intérieur du groupe des pronoms dits 'clitiques' il existe un continuum morphosyntaxique et phonologique ( $c f$. section 2.2.5.2) entre les éléments légers et lourds qui manifestent des comportements différents envers ne (cf. section 2.2.5.2).

En effet, les clitiques lourds comme nous, vous et elle(s) sont accentogènes et incitent parfois à la réalisation de $n e$, tandis que certaines variantes des clitiques légers, comme $[\mathrm{f}]$ pour je et $[\mathrm{s}]$ pour $c e$, conduisent presque inévitablement à son omission ( $c f$. section 4.4.1.2). Par conséquent, la thèse d'une exclusion mutuelle entre ne et 'les clitiques sujet' tout court (conçus comme affixes ou non) ainsi que la formulation d'une règle syntaxique qui exclut ne dans tous les contextes clitiques (cf. Posner 1985: 189, Larrivée 2014) sont difficilement compatibles avec les données de corpus ( $c f$. la vue d'ensemble dans la section 2.2.5.3 et la discussion dans la section 5.1.2).

L'idée d'une coexistence de deux grammaires françaises, sur laquelle repose l'approche diglossique et qui permettrait d'expliquer la variation linguistique en français contemporain (cf. Culbertson 2010, Culbertson/Legendre 2008, Massot 2010, Zribi-Hertz 2011 et aussi Koch/Oesterreicher ${ }^{2} 2011$ dans une optique variationnelle), comporte de nombreux problèmes empiriques et théoriques ( $c f$. la discussion dans la section 5.1.3).

L'approche prosodique semble, par conséquent, une alternative valable aux approches de la contrainte clitique et d'une diglossie française. En effet, elle arrive à intégrer les différents effets des sujets simples et redoublés ainsi que la différence entre les subordonnées et les principales, qui constituent les deux influences les plus importantes sur $\pm n e$, comme l'a montré l'analyse multifactorielle dans la section 4.5.1. De plus, l'influence d'autres contextes syntaxiques (participes présents, gérondifs et constructions infinitives ou impératives) peut être expliquée, selon la même ligne de pensée. Finalement, aux niveaux empirique et théorique, l'approche prosodique est beaucoup moins problématique que les deux autres approches mentionnées ci-dessus, puisqu'elle s'applique aux données sans postuler de règles syntaxiques trop restrictives ou l'existence de deux grammaires du français. 


\subsection{La variation situationnelle $\mathrm{du} \pm n e$ comme épiphéno mène des facteurs prosodiques}

\subsubsection{La nature de la variation situationnelle \pm ne}

Dans les sections précédentes, nous avons montré comment les facteurs linguistiques qui se sont avérés pertinents pour la variable $\pm n e$ ( $c f$. section 4.5) pourraient être intégrés dans une explication prosodique. Cependant, il existe un autre facteur très important pour \pm ne qui se situe au niveau extralinguistique: il s'agit de l'influence de la situation de communication.

Contrairement aux facteurs linguistiques, voire prosodiques, dont l'influence est statistiquement évidente mais peu intégrée dans les approches théoriques de la variation, l'influence de la situation de communication (aussi désignée comme variation stylistique, intrapersonnelle, diaphasique, entre l'immédiat et la distance communicative etc.) est largement reconnue par les chercheurs mais rarement prouvée empiriquement (cf. section 2.4.4.4): Koch/Oesterreicher (2011: 172), par exemple, énumèrent l'absence du ne de négation parmi les caractéristiques saillantes du 'français parlé', c'est-à-dire de la variété de l'immédiat du français contemporain (cf. section 2.3.3).

Comme l'analyse de corpus l'a montré, la première partie de leur supposition, à savoir l'hypothèse selon laquelle l'absence de ne est plus fréquente dans les situations de l'immédiat que dans la distance communicative, est tout à fait justifiée ( $c f$. section 4.3.5). Ceci signifie donc que, même si la deuxième partie de leur affirmation, selon laquelle la présence de ne serait absolue dans la distance communicative (cf. Koch/Oesterreicher ${ }^{2} 2011$ : 172), est loin d'être confirmée (nous n'avons observé qu'un taux de $35 \%$ +ne dans les situations d'examens dans notre corpus, $c f$. section 4.3.5), le fait, reconnu très tôt par Koch/ Oesterreicher (2011), qu'en français moderne les taux de ne (et d'autres variantes linguistiques) dépendent significativement de la situation de communication est indéniable. 
La question cruciale reste donc de savoir pour quelle raison les locuteurs réalisent plus de ne dans la distance communicative. Est-ce parce qu'ils ont accès à une 'grammaire standard' qui est activée dans de telles situations ( $c f$. sections 2.3.3 et 5.1.3)? Parce qu'ils envisagent un certain 'style' formel ou soutenu ( $c f$. sections 2.4.4.4 et 2.3.4)? Ou bien ce phénomène se produit-il suite à une sorte d'hypercorrection, parce que les locuteurs cultivés: «feel that they are speaking badly when they omit ne» ( $c f$. Coveney ${ }^{2} 2002$ : 75 basé sur Désirat/Hordé 1976: 155)?

La première hypothèse, celle des deux grammaires, est empiriquement et théoriquement insoutenable ( $c f$. la discussion dans la section 5.1.3). Par contre, l'adoption d'un certain style ou bien les phénomènes d'hypercorrection restent des explications valables, du moins pour certaines occurrences isolées de $n e$ ( $c f$. sections 5.1.4 et 5.2.4.2).

Il semble également important de noter qu'en France, les questions du bon usage sont traditionnellement discutées avec frénésie, peut-être plus que dans d'autres pays ( $c f$. Rey 2007), ce qui alimente l'hypothèse des hypercorrections.

Georg Bossong (communication personnelle, 28 janvier 2013) décrit cette particularité du français (ou des Français?) de la manière suivante: «[Es ist] ein hervorstechendes typologisches Merkmal des Französischen, dass die interne Variation an der Bruchstelle formal/ informal besonders extrem ausgeprägt ist, auch im Vergleich mit anderen romanischen Sprachen». Effectivement, nous avons vu dans notre corpus que, quant à la variable $\pm n e$, les Français sont beaucoup plus sensibles au facteur de la situation de communication que les Suisses (cf. section 4.3.5). Par conséquent, les hypothèses stylistiques ou d'hypercorrection offrent une bonne explication de certains exemples provenant d'examens oraux en France, mais pas de toute la variation situationnelle $\pm n e$ observée, car elles ignorent le fait que l'omission de ne est plus probable avec un sujet léger que lourd (indépendamment de la situation de communication). Par la suite, nous allons donc présenter une explication alternative, voire complémentaire à ces explications, qui repose sur une observation empirique au fond très simple et qui a surgi de l'analyse multifactorielle ( $c f$. section 4.5.2): il s'agit du fait qu'il 
existe une interaction significative entre les facteurs linguistiques, le type de sujet et le type de phrase, qui influencent \pm ne par leurs caractéristiques prosodiques, et la situation de communication.

\subsubsection{Pourquoi la variation situationnelle \pm ne est-elle un épiphénomène?}

À l'aide de l'analyse des interactions entre les variables pertinentes pour $\pm n e$, nous avons vu que la situation de communication influence la distribution des types de sujet et de phrase, ce qui peut être représenté sous forme d'une structure hiérarchique, $c f$. (184) dans la section 4.5.3, répété sous (242):

(242) La hiérarchie des facteurs qui influencent $\pm n$

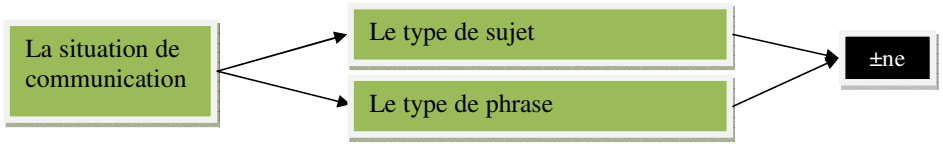

Afin de mieux discerner la hiérarchie illustrée en (242) et de comprendre ses conséquences pour le traitement de la variable \pm ne au niveau théorique, nous allons discuter, dans un premier temps, l'influence exercée par la situation de communication sur la distribution des types de sujet, puis, dans un second temps, celle sur la distribution des types de phrases. Ceci nous permettra de concevoir la hiérarchie en (242) de manière plus détaillée et de mieux comprendre les interdépendances entre les variables en question.

Pour expliciter l'interaction observée entre le type de sujet et la situation de communication, nous avons recours à la notion de structure argumentale préférée (preferred argument structure, PAS), introduite par Du Bois (1987). La PAS désigne une tendance générale selon laquelle les locuteurs intègrent de nouveaux arguments et des éléments lexicaux dans le discours. Du Bois (1987: 819) découvre que dans la langue maya Sakapulteko les locuteurs tendent à respecter quatre contraintes discursives: 
(243) Les contraintes de la structure argumentale préférée selon Du Bois (1987: 819)

a. Évitez l'emploi de plus d'un argument lexical par énoncé

b. Évitez d'exprimer le rôle thématique de l'acteur $\left(\mathrm{A}^{156}\right)$ par un DP lexical

c. Évitez d'intégrer plus d'un nouvel argument par énoncé

d. Évitez d'exprimer le rôle thématique de l'acteur (A) par un élément nouveau

L'existence de la PAS a été attestée dans le domaine de l'immédiat communicatif dans de nombreuses langues ( $c f$. entre autres Lambrecht 1987 pour le français, Ashby/Bentivoglio 1993, 2003 concernant le français et l'espagnol, Schuetze-Coburn 1987 concernant l'allemand, et Smith 1996 concernant l'hébreu).

En ce qui concerne le français, les deux premières contraintes (243) $\mathrm{a}$ et $\mathrm{b}$ peuvent nous aider à interpréter les résultats de l'analyse multifactorielle et à comprendre l'interaction entre la situation de communication et le type de sujet qui influence $\pm n e$. Comme Du Bois (1987) l'a découvert, les locuteurs évitent d'intégrer plus d'un argument lexical dans leur discours et le seul argument lexical ne correspond normalement pas au rôle de l'acteur. Nous retenons donc qu'en français, où le rôle de l'acteur correspond au sujet grammatical dans les constructions actives transitives, il existe une tendance générale à éviter les sujets lexicaux dans le discours.

Ceci signifie que la structure argumentale préférée en français est clitique+verbe+(X) (cf. Lambrecht 1987). Dans un corpus de conversations spontanées d'une famille, Lambrecht (1987: 218) ne trouve que $3 \%$ de sujets lexicaux et conclut que le patron SVO (dans le sens de

156 Comme la langue étudiée par Du Bois (1987) est une langue ergative, il a recours à une classification sémantico-fonctionnelle des arguments du verbe: le rôle thématique A correspond à l'acteur, donc au sujet des verbes transitifs. En français, dans les phrases actives, l'agent et le sujet coïncident obligatoirement. Contrairement à Du Bois (1987), nous n'avons pas analysé la nature des rôles sémantiques tels que l'agent etc., mais la catégorie et le poids phonologique du sujet grammatical, car ceci forme normalement le contexte gauche de la variable \pm ne. Ceci signifie que, même si les catégories appliquées ne sont pas identiques dans les études de Du Bois (1987) et dans la nôtre, les résultats restent comparables. 
sujet lexical+verbe+objet lexical) est tout sauf la structure de base du français:

(244) A look at any stretch of spontaneous spoken discourse in French (as in English, or - as I am tempted to predict - in any other language [...]) is sufficient to show that the SVO clause pattern is not the predominant pattern at the level of surface structure. (Lambrecht 1987: 218)

Cette évaluation de Lambrecht (1987) correspond parfaitement aux résultats obtenus dans notre corpus ( $c f$. section 4.5.2.1): le nombre de sujets lexicaux et donc prosodiquement lourds est généralement restreint, mais la majorité des occurrences de ce type de sujet se trouve dans la distance communicative. Par ailleurs, nous avons vu que la longueur moyenne des sujets est de deux segments (par exemple je [3ə]), mais que dans la distance communicative il y a un grand nombre de sujets complexes, forcément lexicaux, qui manifestent une longueur de jusqu'à 30 segments (cf. le renseignement sur le déroulement de ses journées, 0180).

Conformément aux observations de Koch/Oesterreicher $\left({ }^{2} 2011\right.$ : 105) concernant les caractéristiques universelles de l'immédiat communicatif et aux suggestions de Dufter/Stark (2007: 120), qui ont été les premiers à tirer une parallèle entre la PAS et l'absence de ne, nous pouvons aller encore un peu plus loin que Du Bois (1987) et Lambrecht (1987) l'ont fait: l'interaction entre la situation de communication et la distribution des types de sujet que notre analyse a montrée ( $c f$. section 4.5.2.1) permet de supposer que la PAS est plus articulée dans l'immédiat communicatif que dans la distance communicative. En d'autres termes, la tendance à l'emploi des sujets légers est particulièrement forte dans l'immédiat communicatif.

Considérons maintenant l'impact de cette observation sur la variable \pm ne. Comme nous le savons grâce à de nombreuses analyses de corpus ( $c f$. sections 2.2.5.1 et 4.4.1.2), les clitiques légers empêchent la réalisation de la particule ne. Ceci est dû, selon nous, à leur faiblesse prosodique qui les empêche de porter l'accent initial typique de l'AP français prototypique ( $c f$. section 5.2), ce qui donne lieu à l'omission de ne comme stratégie de compensation ( $c f$. section 5.2.4.1). 
Par conséquent, la variation situationnelle des taux de \pm ne ne s'expliquerait pas tout simplement par le fait que, dans l'immédiat communicatif, l'emploi de ne serait réfuté par les locuteurs et que dans la distance communicative il serait préféré, ce qui en soi ne représente qu'une constatation des faits (comme on la trouve souvent en sociolinguistique par rapport au 'style', $c f$. Bell 1984, 2001, Finegan/Biber 2001, Labov 2001 ou en linguistique variationnelle, $c f$. Forner 2005, Koch/Oesterreicher ${ }^{2} 2011$ ).

Par contre, comme la figure en (245) le montre, la variation situationnelle observée s'explique à un niveau plus profond, à savoir par une différence universelle entre l'immédiat et la distance: la PAS. Dans l'immédiat communicatif, elle opère de manière plus forte que dans la distance communicative, comme l'étude de corpus l'a montré.

(245) L'interaction entre la PAS et les taux de $\pm n e$ dans l'immédiat et la distance communicative

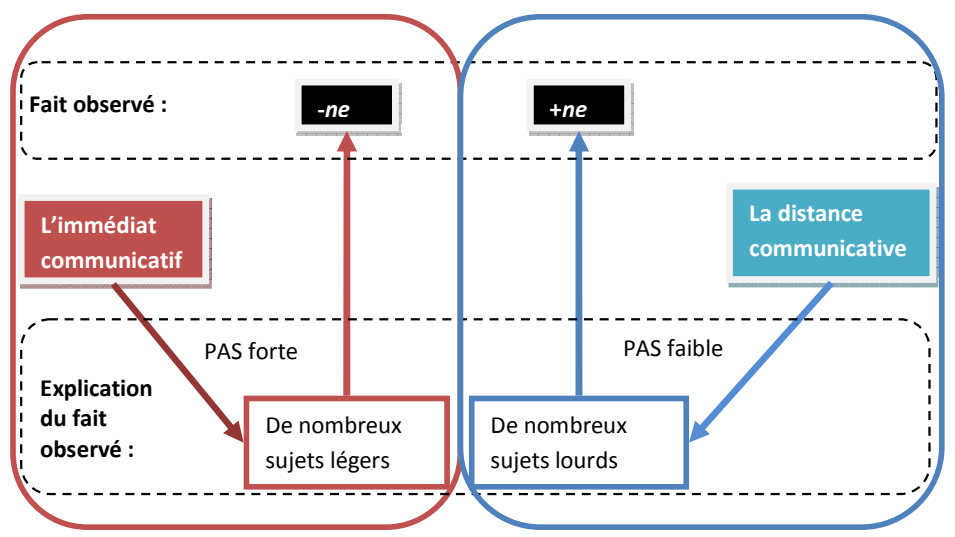

Ceci implique que le taux des sujets légers dans le discours de l'immédiat est généralement très élevé. Dans la distance communicative, en revanche, la PAS opère à un moindre degré: ici, le taux des sujets lourds est plus élevé.

La PAS est donc responsable de la variation situationnelle de $\pm n e$ : tandis qu'une grande présence de sujets clitiques légers dans les situa- 
tions de l'immédiat y déclenche l'absence presque totale de ne, la présence un peu plus forte de sujets lourds dans la distance communicative y empêche la perte de $n e$.

Comme la distribution des types de sujet, la distribution du deuxième facteur linguistique pertinent pour \pm ne, à savoir celle des types de phrase, a elle aussi été décrite comme universellement dépendante de la situation de communication:

(246) Aus dem geringen Planungsgrad folgen weitere Merkmale des Diskurses in konzeptioneller Mündlichkeit. Der Diskurs zeichnet sich hier durch Prozesshaftigkeit und Vorläufigkeit aus. Dies führt zum einen zu sparsamer Versprachlichung, zum anderen zu einer häufig extensiven, linearen und aggregativen Gestaltung ('unvollständige' Äußerungen, Parataxe etc.). (Koch/Oesterreicher ${ }^{2} 2011: 12$, notre soulignement)

Koch/Oesterreicher (22011) (cf. aussi Finegan/Biber 2001 : 252 et Cheshire/Stein 1997) comptent la présence élevée des parataxes parmi les caractéristiques universelles du langage de l'immédiat communicatif. Cette supposition est confirmée par notre corpus: même si les principales sont prédominantes dans toutes les situations de communication, les subordonnées sont beaucoup plus fréquentes dans la distance communicative ( $c f$. section 4.5.2.2). Nous savons par ailleurs que les subordonnées manifestent des taux significativement élevés de $n e$ ( $c f$. section 4.4.5.1), car leurs caractéristiques syntaxico-prosodiques 'protègent' la particule ne ( $c f$. section 5.2.4.5).

Dans le but de différencier l'influence des facteurs discutés ci-dessus sur $\pm n e$, nous pouvons donc affiner la hiérarchie présentée en (242), comme illustré dans la figure (247). Nous voyons trois 'colonnes' : à gauche (en vert) figurent les variables qui influencent prosodiquement $\pm n e$ et à droite les variantes qui correspondent d'un point de vue quantitatif à l'immédiat (en rouge) et à la distance communicative (en bleu). Les flèches verticales indiquent les relations de dépendance: la situation de communication influence la distribution des variantes des types de sujet et de phrase, qui, à leur tour, influencent la présence de ne. Dans l'immédiat communicatif, la présence élevée de sujets légers et de phrases principales provoque l'absence fréquente de ne $(5 \%+n e, c f$. 
section 4.3.5). Par contre, dans la distance communicative, la présence élevée de sujets lourds et de phrases subordonnées donne lieu à une plus grande fréquence de $n e(35 \%+n e, c f$. section 4.3.5).

(247) La hiérarchie détaillée des facteurs qui influencent $\pm n e$

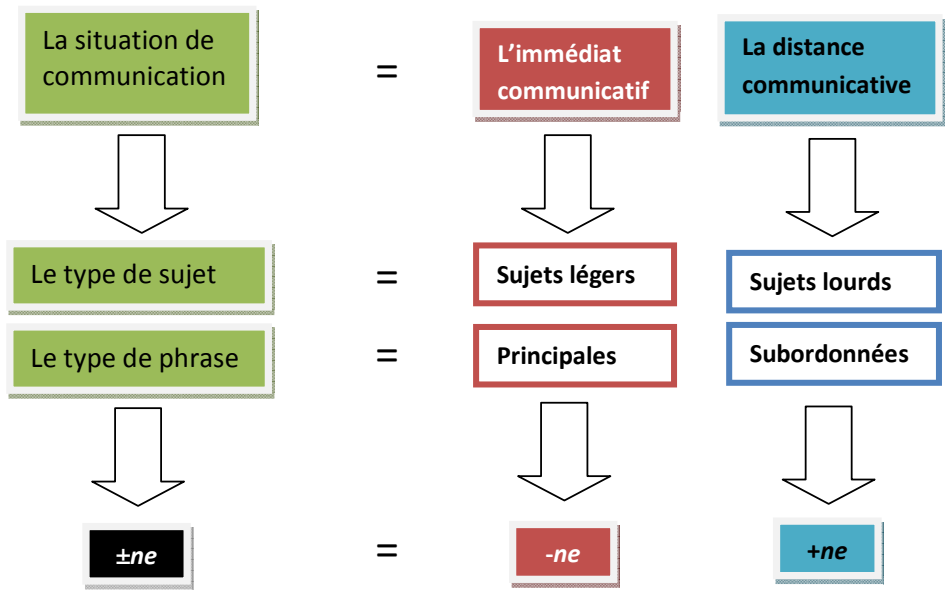

C'est cette interaction des facteurs linguistiques et extralinguistiques, éventuellement quelque peu sous-estimée par la recherche sur la variable $\pm n e$, qui est, à notre avis, à l'origine de la variation situationnelle de $\pm n e$. Cette variation est donc, en dernière instance, un épiphénomène de la distribution des variantes linguistiques à travers les situations de communication.

\subsubsection{D’autres facteurs pertinents liés à la situation de communication}

Il existe bien évidemment d'autres facteurs à considérer: tout d'abord, le fait que la présence de ne est au fond un épiphénomène des facteurs linguistiques n'empêche pas que les locuteurs (surtout les Français, comme nous l'avons vu dans la section 4.3.5, ou ceux qui ont une formation académique ou exercent une fonction éducative) produisent aussi délibérément et intentionnellement des ne d'hypercorrection dans la 
distance communicative, même si le contexte syntaxico-phonologique n'y incite pas. Ceci reflète, en premier lieu, le prestige qui est associé à la variante $+n e$, surtout en France et un peu moins en Suisse ( $c f$. section 4.3.5), dans les situations de distance communicative ${ }^{157}$.

Les hypercorrections pourraient également refléter les différentes quantités perçues de $\pm n e$ dans les discours de l'immédiat et de la distance communicative que l'on trouve dans les corpus. En d'autres termes, les locuteurs perçoivent la plus grande présence de ne dans la distance communicative et essaient de la reproduire, car elle leur semble caractériser les discours de distance.

Simon et al. (2010) ont testé la réalisation des phonostyles, c'est-àdire l'adaptation de productions langagières aux exigences de situations déterminées ( $c f$. aussi Lindblom 1990) dans sept activités de parole:

(248) Les activités de parole examinées par Simon et al. (2010)

\begin{tabular}{|ll|}
\hline Publique/préparé & Privé/spontané \\
\hline Lecture à haute voix & demande d'itinéraire \\
Discours de chefs d'état & conversation entre locuteurs intimes \\
Nouvelles radiophoniques & \\
Interview radiophonique & \\
Conférence scientifique & \\
\hline
\end{tabular}

Les analyses de Simon et al. (2010) montrent qu'il y a des variations subtiles entre les situations testées, mais que l'un des critères s'avère particulièrement important: le caractère publique/préparé ou privé/ spontané de la production langagière. Tandis que les paroles des journalistes radio, la lecture neutre et, à un moindre degré, les discours politiques représentent un phonostyle relativement homogène, normé et identifiable, les discours spontanés manifestent une variation interpersonnelle plus grande.

157 Shana Poplack et Johanne S. Bourdages observent le même phénomène au Canada (cf. le descriptif du projet de recherche Norms and variation in French: the competing roles of school, community and ideology disponible sous : $<\mathrm{http}: / /$ www.sociolinguistics.uottawa.ca/projects/norms.html $>$ ). 
Les corrélats phonétiques qui sont particulièrement intéressants en relation à la question du \pm ne sont le débit d'articulation et la densité accentuelle. Le premier critère désigne le nombre de syllabes produites par seconde en excluant les pauses. Celui-ci ne peut pas être rapporté à un style en particulier, mais deux genres à support écrit, à savoir la parole lue et le journal parlé, se caractérisent par une très haute vitesse (6,13 et 5,87 syllabes p.s., cf. Simon et al. 2010: 75).

Le deuxième critère fournit une approximation de la longueur des groupes accentuels: une proportion élevée de syllabes proéminentes indique des groupes plutôt courts et vice versa (cf. Simon et al. 2010: 76). Les groupes accentuels les plus longs sont observables dans deux styles assez différents: la parole lue et les conversations intimes. Dans la lecture, ce fait est probablement lié à la vitesse de production. Par contre, dans les conversations, qui ont un débit moyen, la longueur des groupes accentuels indique le rapprochement de davantage de syllabes à une unité prosodique. Les unités accentuelles contiennent donc plus de syllabes inaccentuées, dont certaines, comme le clitique ne et d'autres syllabes contenant un schwa, sont instables.

Leur tendance à la disparition dans le langage spontané et conversationnel est donc explicable par la longueur élevée des groupes accentuels. Quant à la question du $\pm n e$, le rapprochement des syllabes à l'intérieur des unités accentuelles, qui va au détriment des syllabes inaccentuées, est un facteur ultérieur qui s'ajoute aux autres facteurs prosodiques qui freinent la réalisation de la particule de négation dans le domaine de l'immédiat.

\subsubsection{Les implications théoriques pour le traitement de la variable \pm ne}

En guise de conclusion intermédiaire sur la variation situationnelle de $\pm n e$, nous notons qu'elle est avant tout un épiphénomène de la distribution des types de sujet et de phrase. Même si d'autres facteurs, comme l'hypercorrection ou les phonostyles, peuvent eux aussi influencer la variable \pm ne au niveau situationnel, ce sont surtout les sujets légers et les phrases principales qui empêchent la présence de ne dans l'immédiat 
communicatif, tandis que les sujets lourds et les subordonnées provoquent sa réalisation dans la distance communicative.

Si la variation situationnelle de \pm ne est un épiphénomène des facteurs linguistiques, il faut se demander quelles sont donc les conséquences pour le traitement sociolinguistique et variationnel de cette variable.

Premièrement, nous avons vu que la variable $\pm n e$ (comme éventuellement d'autres variables dites 'sociolinguistiques') est à traiter comme une sorte de puzzle: seule la combinaison des pièces déjà connues mais dispersées à travers différentes approches permet de composer une image intégrale de la variable. Dans notre étude, il s'agissait de combiner les pièces suivantes:

1. L'analyse (multifactorielle) d'un corpus (phonétiquement transcrit) (cf. Armstrong/Smith 2002, Ashby 1976, 1981, 2001, Coveney 2002 etc.),

2. Les faits théoriques et typologiques: les approches variationnelles (cf. Koch/Oesterreicher ${ }^{2} 2011$ ), les analyses des clitiques dans les langues romanes en tant que continuum (cf. Bossong 1998) et les études de la prosodie française ( $c f$. Di Cristo 1998, Jun/Fougeron 2000 etc.),

3. Les tendances discursives universelles comme la PAS ( $c f$. Du Bois 1987, 2003, Lambrecht 1987).

Ainsi, nous avons établi que la raison principale pour l'absence de ne dans les discours de l'immédiat en français moderne se trouve au niveau linguistique.

Il n'y a donc dans notre corpus aucune motivation à classer \pm ne comme variable sociolinguistique, comme il est de pratique courante (cf. Armstrong/Smith 2002: 23, Coveney ${ }^{2} 2002$ : 55, Gadet 1997b, Labov 1972 b et la discussion dans la section 5.1.1).

Deuxièmement, il est improbable que la variable \pm ne indique une variété ou grammaire du français de l'immédiat (comme le soutiennent Culbertson 2010, Culbertson/Legendre 2008, Massot 2010, Mensching 2008, Koch/Oesterreicher ${ }^{2} 2011$ et Zribi-Hertz 2011), car elle dépend de tendances discursives valables dans de nombreuses langues: tout 
comme la distribution des types de sujet et de phrase, la variation $\pm n e$ dépend elle-même des caractéristiques universelles de l'immédiat communicatif et non de celles propres à une variété du français.

Ceci n'exclut pourtant pas que la variable \pm ne soit perçue comme indiquant une telle variété, mais une explication linguistique proprement dite de cette variable ne doit pas se contenter de refléter tout simplement la perception des locuteurs. Une réévaluation de la portée des influences linguistiques, voire prosodiques sur $\pm n e$, comme nous l'avons proposée ici, peut donc entraîner une reconsidération des méthodes d'investigation et des caractéristiques sociolinguistiques ou variationnelles attribuées à la variable $\pm n e$.

Finalement, nous tenons à souligner que l'explication que nous proposons n'est pas incompatible avec d'autres approches de la variable $\pm n e$. Dans la section suivante, nous allons proposer quelques pistes qui permettent de combiner certains aspects des approches existantes de $\pm n e$ avec la nôtre.

\subsection{Perspectives}

\subsubsection{L'explication prosodique et d'autres approches de la variable \pm ne}

Nous avons montré dans la section 5.1 que ni les quatre approches de la variation \pm ne présentes dans la littérature scientifique, ni celle du contact linguistique, surgie lors de notre analyse, ne sont pas autosuffisantes pour expliquer la variation en question. Toutefois, elles ne seront pas simplement réfutées. Au contraire, vu que chacune de ces approches comporte des éléments qui peuvent être combinés avec l'explication prosodique, nous proposons ici quelques pistes pour des futurs travaux de recherche.

Premièrement, même si, contrairement à Armstrong/Smith (2002: 23) et Coveney (2002: 55) (cf. section 2.3.1), nous contestons le traitement de $\pm n e$ comme une variable sociolinguistique tout court (cf. les 
discussions dans les sections 5.1.1 et 5.3), il y a indubitablement certaines différences de nature sociolinguistique quant à \pm ne en fonction la provenance géographique ( $c f$. sections 2.2.4.1 et 4.3.1) et de l'âge ( $c f$. sections 2.2.4.2 et 4.3.2). À un moindre degré, nous avons également découvert un comportement particulier de certains groupes professionnels, comme la fidélité des professeurs à la norme ( $c f$. sections 2.2.4.3 et 4.3.3).

Certains aspects sociolinguistiques sont également fortement pertinents pour la prosodie. En diatopie, il y a, par exemple, une grande différence entre le nord et le sud de la France en ce qui concerne l'articulation du schwa, qui est plus répandue dans le Midi ( $c f$. Durand et al. édd. 2009), ce qui influence très probablement la réalisation de ne. De plus, il y a une tendance à l'accentuation initiale en Suisse romande ( $c f$. Woehrling et al. 2008: 787), ce qui correspond parfaitement aux taux plus bas de ne trouvés dans cette région, vu que l'accent initial est difficilement compatible avec $n e^{158}$.

Les différences intonatives de génération en génération ont également été montrées. Bedijs (2012: 221), par exemple, observe à partir des années 90, dans le 'langage des jeunes' mis en scène dans un corpus filmique, les caractéristiques phonologiques suivantes: un débit de parole extrêmement élevé, l'élision des voyelles inaccentuées, qui provoque la formation des complexes consonantiques (ex. je te jure [ $\mathrm{t} \int \mathrm{yr}$, $p$ (u)tain [pt $\tilde{\varepsilon}$ ], $c f$. Bedijs 2012: 222-223), et une tendance à l'emploi d'un accent (éventuellement emphatique) sur l'une des premières syllabes d'un groupe accentuel, par exemple dans ces pétasses [se'pe:tas] (cf. Bedijs 2012: 222). En revanche, Gerstenberg (2011: 234), qui note pour les locuteurs âgés d'entre 70 et 90 ans des taux de ne très élevés, trouve que Les rares contextes où il est omis sont précisément ceux qui suivent un pronom clitique. Par conséquent, nous constatons suivant Bedijs (2012: 222-223) et Gerstenberg (2011 : 234) que la tendance prosodique à l'omission de ne, déjà présente chez les personnes âgées, est intensifiée par les caractéristiques prosodiques des jeunes. Dans l'ensemble,

158 Je remercie Aurélia Robert-Tissot de m'avoir fait remarquer cet aspect. 
les différences sociolinguistiques diatopiques et intergénérationnelles sont donc aussi interprétables comme des différences intonatives.

Deuxièmement, quant à l'incompatibilité entre ne et d'autres clitiques (cf. Ashby 1977, Culbertson/Legendre 2008, Larrivée 2014, Posner 1985: 189 et la section 2.3.2), il y a effectivement un inventaire restreint de formes presque lexicalisées, comme je sais pas [ $\int \varepsilon \mathrm{pa}$, c'était [ste] ou il y a [ja], qui excluent catégoriquement la présence de ne ( $c f$. section 4.4.1.2). Dans ces cas, ou dans les séquences proclitiques (cf. section 5.2.4.2), les clitiques sujet sont tellement réduits qu'ils ne forment plus de syllabe complète et sont agglutinés à la forme verbale. Ces formes réduites sont en concurrence avec les variantes 'pleines' des clitiques, par exemple [3ə] ou [sə], qui permettent non seulement l'insertion de $n(e)$ entre le clitique sujet et la forme verbale (cf. [3ə.(nə). $\mathrm{s} \varepsilon . p a]$ ou $[\mathrm{s}($ ə.n)e.te.pa]), mais également sa réalisation sous forme de syllabe CV: [nə] ou par exemple [ne] dans ce n'était pas.

Par conséquent, même si nous n'observons aucune exclusion mutuelle de type 'mécanique' ou idiosyncratique entre les clitiques et ne (comme le suggèrent par exemple Culbertson/Legendre 2008), un fait phonotactique essentiel émerge de ces observations: la qualité syllabique du clitique sujet est cruciale pour la qualité syllabique, voire pour la réalisation de $n e$. Les réductions phonologiques lexicalisées, comme je sais pas, sont donc parfaitement en ligne avec l'explication prosodique proposée ici.

Troisièmement, même si l'approche diglossique ( $c f$. Culbertson 2010, Culbertson/Legendre 2008, Massot 2010, Mensching 2008, Koch/ Oesterreicher ${ }^{2} 2011$, Zribi-Hertz 2011 et la section 2.3.3), qui prévoit deux grammaires du français, n'est pas soutenable face aux données du corpus ( $c f$. section 5.1.3), l'idée d'une sensibilité de certains locuteurs par rapport à la situation de communication s'est avérée vraie. Chez certains individus (surtout de France), nous avons observé une véritable alternance stylistique d'une situation de communication à l'autre, qui concerne notamment la variable $\pm n e$ ( $c f$. section 4.3.5). De plus, des études prosodiques ont confirmé qu'il y a des différences situationnelles entre différents phonostyles ( $c f$. section 5.3). Il est donc tout à fait convainquant de supposer qu'un sous-ensemble de locuteurs change son 
comportement prosodique et linguistique en fonction de la situation de communication. Ceci s'observe dans le débit de parole, dans la constitution des unités prosodiques et dans l'articulation des schwas (qui conditionne la réalisation de ne), sans pour autant entraîner nécessairement la proclamation de deux grammaires distinctes.

Quatrièmement, l'approche pragmatique ( $c f$. Breitbarth/Haegeman à paraître, van Compernolle 2008a,b, 2009 Fonseca-Greber 2007, Poplack/St-Amand 2009, Sankoff/Vincent 1980 et la section 2.3.4), qui explique surtout l'emploi de $+n e$ dans des contextes 'sérieux' ou emphatiques, s'avère particulièrement utile pour l'explication des occurrences inattendues de $+n e$. Il s'agit notamment des occurrences (très rares mais existantes) qui ne sont explicables ni en fonction du contexte grammatical ni comme des cas d'hypercorrections.

Contrairement au critère du 'discours institutionnel' invoqué par Fonseca-Greber (2007), qui nous semble peu concret et donc difficile à appliquer, les contextes emphatiques manifestent un corrélat prosodique clairement défini par van Compernolle (2009: 11) qui permet l'explication des exemples comme (199) (répété sous (249)), qui ne pourraient pas l'être autrement.

(249) moi Pierre Boulle je veux pas / JE - NE - VEUX-PAS / je l'ai pas lu

Même si les raisons de l'emploi de +ne dans ces cas-là sont surtout pragmatiques, elles vont de pair avec une intonation particulaire (ralentissement du débit de parole et accentuation syllabique), qui permet l'assignation exceptionnelle de $\mathrm{Hi}$ au clitique ne. Il s'agit donc, en dernière instance, une fois de plus d'un fait prosodique, plus précisément de l'intonation emphatique, qui permet la réalisation de ne.

Finalement, le contact linguistique ( $c f$. sections 4.3 .4 et 5.1.5) implique également un contact prosodique. La vue d'ensemble des systèmes accentuels en (250) révèle deux différences principales entre le français et les langues de contact qui provoquent dans notre corpus une absence totale de ne: il s'agit de l'absence de l'accent de mot et de l'accent contrastif en français. 
(250) Les systèmes intonatifs du français des langues en contact

\begin{tabular}{|lcccc|}
\hline Langue & Accent de mot & Accent de phrase & $\begin{array}{l}\text { Accent de focalisation } \\
\text { contrastive }\end{array}$ & \\
\cline { 1 - 4 } Français & - & + & -159 & Di Cristo 1998 \\
\hline Anglais & + & + & + & Hirst 1998 \\
Arabe & + & + & + & Benkirane 1998 \\
Allemand & + & + & + & Gibbon 1998 \\
turc & + & + & + & Nash 1973 \\
\hline
\end{tabular}

Même si les systèmes intonatifs en question sont beaucoup plus complexes que ce que la grille en (250) ne permet de montrer, la particularité du système français par rapport aux autres systèmes saute à l'œil. Il est donc envisageable que non seulement le contact syntaxique, mais également le contact prosodique puisse avoir une influence sur la modalité de la variation linguistique en français. Vu qu'une 'prosodie du contact' se trouve actuellement dans une phase initiale, nous ne proposons aucune solution finale à la question de l'influence du contact linguistique sur la variation \pm ne et nous nous limitons à signaler cette piste pour de futurs travaux de recherche.

\subsubsection{Quel futur pour le ne de négation?}

Après une considération prudente des traces historiques de l'évolution de la négation en français (résumées dans la section 2.1.2) et des résultats de l'analyse de corpus (dans les sections précédentes), nous pouvons oser, avec précaution, répondre à la question l'avenir de ne: nos données suggèrent que le ne de négation continue effectivement d'exister, dans certains contextes, même dans le français de l'immédiat. Nous

159 Effectivement, la focalisation contrastive par moyens intonatifs par exemple $L e$ profeSSEUR a la clé pas l'étudiant (cf. Di Cristo 1998: 210) n'est pas complètement exclue en français. Toutefois, il y a un certain consensus en ce qui concerne le fait que le français préfère une transposition syntaxique du focus, par exemple par une construction clivée comme: C'est le profeSSEUR qui a la clé pas l'étudiant (cf. par exemple Dufter 2007). 
nous n'attendons donc à aucune perte totale de ne dans les décennies à venir.

L'existence de ne est largement limitée aux contextes qui le favorisent prosodiquement, comme les sujets lourds et les constructions subordonnées ( $c f$. section 5.2.4), mais dans ces environnements, sa réalisation est assez robuste, et ce indépendamment de la situation de communication: nous avons trouvé entre $68 \%$ et $100 \%$ +ne avec les sujets lexicaux, les noms propres et les pronoms indéfinis ( $c f$. section 4.4.1) et $48 \%+n e$ dans les constructions relatives ( $c f$. section 4.4.5.1).

Le fait que les contextes favorisant ne, comme les sujets lourds, sont très rares dans le langage spontané ( $c f$. Du Bois 1987, 2003), surtout dans les situations de l'immédiat, a un effet considérable dans les corpus ( $c f$. section 5.3): il véhicule l'illusion que le ne a presque déjà disparu du français moderne dit 'oral' (cf. Ashby 1976, 1981, 2001, Armstrong/Smith 2002, Coveney ${ }^{2} 2002$ ), ce qui, comme nous l'avons vu, n'est pas le cas.

$\mathrm{Au}$-delà des contextes qui retiennent ne prosodiquement, il y a (surtout en France) des cas d'hypercorrection ou d'adaptation stylistique à une situation de distance qui peuvent mener à la réalisation de ne (cf. van Compernolle 2008a,b, 2009 Fonseca-Greber 2007 et la section 5.3) ainsi que certaines occurrences plutôt rares d'un ne emphatique ( $c f$. section 5.1.4), qui sont éventuellement comparables à l'emploi de la particule emphatique en en flamand de l'ouest ( $c f$. Breitbarth/Haegeman à paraître).

Résumons maintenant ce que nous pouvons conclure de ces considérations quant à l'évolution de la négation en français ( $c f$. section 2.1.2.2). En ce qui concerne le cycle de Jespersen (cf. Jespersen 1917, 1924), le français se trouve donc toujours dans la quatrième phase du cycle, c'està-dire que deux formes de l'expression de la négation de phrase, à savoir ne...pas et pas, sont en concurrence. Néanmoins, nous avons également vu qu'il ne s'agit pas d'une 'variation libre' (si une telle chose existe, cf. Gadet 1997a,b pour une discussion), mais que la réalisation du ne dépend des caractéristiques linguistiques des énoncés négatifs.

Nous rappelons qu'il existe, d'une part, une hypothèse qui décrit l'évolution de la négation en français comme un changement linguis- 
tique rapide et tardif ayant débuté au $19^{\mathrm{e}}$ siècle et qui aboutirait à une perte totale de ne ( $c f$. Ashby 2001, Martineau/Mougeon 2003), et d'autre part, celle qui l'interprète plutôt comme une variation linguistique plus ou moins stable depuis le $17^{\mathrm{e}}$ siècle environ ( $c f$. Dufter/Stark 2007, Martineau 2011, Poplack/St-Amand 2009) et qui garde la variable $\pm n e$ comme partie du système linguistique.

Nos résultats, qui ressemblent à ceux obtenus par Dufter/Stark (2007) pour le français du $17^{\mathrm{e}}$ siècle, suggèrent que la variable \pm ne est conditionnée par les mêmes facteurs depuis plusieurs siècles. Les sujets lourds incitent à la réalisation du ne déjà depuis l'époque du Dauphin, comme l'étude de Dufter/Stark (2007) sur le corpus d'Héroard (cf. Ernst 1985) l'a montré.

La grande majorité des données semble donc mieux décrite par l'hypothèse d'une variation ancienne et relativement stable qui s'étend jusqu'au français moderne ( $c f$. Dufter/Stark 2007, Martineau 2011, Poplack/St-Amand 2009) que par une perte abrupte de ne au cours des deux derniers siècles (cf. Martineau/Mougeon 2003, Ashby 2001). Cela implique qu'aujourd'hui la particule ne fait toujours partie de la grammaire de la majorité des francophones contemporains, donc de celle des locuteurs européens prototypiques classés comme appartenant au groupe A, qui manifestent de la variation \pm ne en fonction des facteurs linguistiques.

Ceci dit, il nous reste à revenir brièvement sur le groupe de locuteurs suisses, classés comme type B, qui ne manifestent que la négation monopartite et ceci apparemment indépendamment du contexte syntacticoprosodique et situationnel ( $c f$. section 4.2.2). La question reste de savoir s'il est possible que ce sous-ensemble de locuteurs ait déjà complètement généralisé l'absence de ne en français moderne. Vu la rareté des contextes qui favorisent $+n e$, surtout dans l'immédiat communicatif, notre analyse de corpus ne produit pas de résultats fiables en ce qui concerne la compétence de ces locuteurs. Comme nous l'avons déjà suggéré dans la section 4.2.2, il serait intéressant de soumettre ces locuteurs à des tests d'intuition ( $c f$. Coveney 1998, Meisner 2013) afin de déterminer le statut de la particule ne dans leurs grammaires. Cette question devra donc, elle aussi, être abordée dans de futurs travaux de recherche. 
Somme toute, pour la grande majorité des francophones contemporains restants, il semble clair qu'ils continuent à employer $+n e$ de manière relativement stable dans les contextes qui le favorisent prosodiquement, même si ceux-ci sont assez rares dans le langage spontané.

\subsection{Résumé du chapitre 5}

Nous avons montré dans le sous-chapitre 5.1 que les approches sociolinguistiques, syntaxiques, diglossiques, pragmatiques et de contact linguistique de la variation \pm ne représentent des pistes intéressantes sans pourtant offrir des explications intégrales du phénomène en question.

D'abord, les analyses de \pm ne comme variable sociolinguistique (cf. Armstrong/Smith 2002, Ashby 1976, 1981, 2001, Coveney ${ }^{2} 2002$ etc.) produisent des résultats contradictoires et laissent beaucoup de questions sans réponse. Deuxièmement, la thèse d'une incompatibilité rigide entre ne et d'autres clitiques ( $c f$. Posner 1985, Larrivée 2014) est indéfendable face aux données du corpus et ignore le caractère prosodico-syntaxique continuel du paradigme des pronoms sujet ( $c f$. section 2.2.5.2). Troisièmement, les données suggèrent clairement que l'existence d'une seule grammaire du français qui permet de la variation contextuelle est plus probable que la coexistence de deux grammaires en situation diglossique (qui est soutenue par Culbertson 2010, Culbertson/Legendre 2008, Massot 2010, Mensching 2008, Koch/Oesterreicher 22011 et Zribi-Hertz 2011). Quatrièmement, les explications pragmatiques des occurrences $+n e$ ( $c f$. van Compernolle 2008a,b, 2009, FonsecaGreber 2007) expliquent des cas isolés, mais ne sont pas généralisables. Finalement, l'influence du contact linguistique sur la variation linguistique du français est encore trop peu explorée pour servir d'explication indépendante.

Dans le sous-chapitre 5.2, nous proposons une explication prosodique qui pourrait offrir une solution autosuffisante à la question de 
$\pm n e$. Elle nous permet d'expliquer aussi bien l'absence de ne avec les sujets légers, redoublés et les séquences proclitiques que sa présence avec les sujets lourds et dans les constructions subordonnées, qui ont été montrées lors de l'analyse multifactorielle dans le chapitre précédent.

L'idée défendue ici est la suivante: l'omission fréquente de la particule ne avec les sujets clitiques est due, grosso-modo, à l'incompatibilité entre l'accent initial secondaire du français contemporain (selon Jun/Fougeron 2000) et les groupes accentuels comportant plus d'un seul proclitique. Comme les clitiques ne peuvent pas porter d'accent, l'omission du ne ainsi que d'autres stratégies de compensation prosodique interviennent afin de maintenir tout de même l'alternation des syllabes fortes et faibles (cf. principe eurythmique Dell 1984, Di Cristo 1998: 197).

Cette analyse est implémentée à l'aide du modèle prosodique selon Jun/Fougeron (1995, 2000, 2002) et Fougeron/Jun (1998), qui décrivent les caractéristiques intonatives générales du français de la manière suivante: le français possède un accent final primaire $\left(\mathrm{H}^{*}\right)$ sur la dernière syllabe pleine et un accent initial secondaire (Hi) facultatif sur l'une des premières syllabes d'un groupe accentuel (AP). Le placement concret des accents varie en fonction du nombre et de la nature (grammaticale ou lexicale) des morphèmes en question, du débit de parole et du style individuel du locuteur. Dans les groupes accentuels qui commencent par un ou plusieurs clitiques, sur lesquels Hi ne peut pas être réalisé, l'accent tombe obligatoirement sur la première syllabe du verbe. En fonction du nombre de proclitiques, ceci peut provoquer un éloignement considérable de la structure intonative basique du français, qui comporte généralement l'accent initial sur une des premières syllabes (cf. Welby 2003). Un tel déplacement de l'accent initial à droite serait accompagné d'un rapprochement entre l'accent initial et final vers la fin de l'AP. Une telle accumulation d'accents contredit le principe eurythmique ( $c f$. Dell 1984, Di Cristo 1998: 197), qui favorise l'alternance des syllabes fortes et faibles.

Nous soutenons que la tendance à la réalisation de la structure intonative basique du français est plus forte que la tendance à la réalisation des clitiques. Plus précisément, un ensemble de stratégies compensatrices 
permet le (re-)déplacement de Hi vers le début du groupe accentuel comportant plus d'un proclitique: ceci est le plus visible dans le cas du ne négatif, souvent complètement supprimé, mais les réductions ou fusions des autres proclitiques s'observent aussi souvent. Une telle réduction des séquences proclitiques mène à des structures presque lexicalisées, dans lesquelles la réalisation de ne est exclue (cf. je sais pas $\left.\rightarrow\left[\int \varepsilon p a\right]\right)$.

Les sujets redoublés (ex. moi je l'aime pas du tout, 1660) excluent eux aussi ne prosodiquement, car ils comprennent presque toujours un élément léger (le clitique coréférentiel à l'XP lourd), qui occupe obligatoirement une position inaccentuée à l'intérieur de l'AP. Celui-ci est donc en concurrence directe avec ne, ce qui donne lieu à l'exclusion de ce dernier. De plus, dans la majorité des sujets redoublés, le pronom de reprise est la variante monosegmentale [s] du pronom démonstratif ce, qui s'est montrée généralement incompatible avec ne ( $c f$. section 4.4.1.2).

En revanche, si un sujet lourd se trouve en position initiale de l'AP, Hi peut être réalisé dans cette position et l'application des stratégies de compensation, comme l'omission de ne, est superflue. Les sujets lourds 'protègent' donc la particule ne de l'omission au niveau prosodique et ceci vaut également pour la présence d'une conjonction de subordination dans une phrase, qui alterne la structure prosodique en favorisant la présence de $n e$.

Ensuite, la variation de $\pm n e$ avec les gérondifs, les participes présents, les infinitifs niés ainsi que dans les impératives négatives comportant des proclitiques (ex. \pm n'y vas pas) semble explicable à travers la même approche prosodique que celle dans les constructions finies à l'indicatif. Pour comprendre l'impossibilité de +ne dans les impératives comportant des enclitiques (par exemple (*ne) vaz-y pas), il faut retourner aux données de l'ancien français et du français classique. Ces séquences peuvent être identifiées comme des vestiges de la loi Tobler/ Mussafia ( $c f$. Hirschbühler/Labelle 2000), qui excluait le placement des clitiques dans la position initiale en ancien français.

Par la suite, dans le sous-chapitre 5.3, une considération détaillée de la distribution des deux facteurs linguistiques les plus importants pour $\pm n e$, à savoir les types de sujet et de phrase, nous a amené à 
conclure que l'omission de ne est un épiphénomène de la distribution des facteurs linguistiques, qui, à son tour, est conditionnée par des tendances discursives universelles.

Par exemple, la distribution des types de sujet remonte à une tendance discursive découverte par Du Bois (1987), à savoir la structure argumentale préférée (angl. preferred argument structure, PAS). Ainsi, la variation situationnelle de $\pm n e$ s'explique par la PAS : dans l'immédiat communicatif, le taux des sujets légers (qui empêchent habituellement la réalisation de $n e$ ) est très élevé. Dans la distance communicative, par contre, c'est le taux des sujets lourds (favorisant la réalisation de ne) qui est clairement élevé. Ainsi, le réseau complexe de variables linguistiques et extralinguistiques qui influencent \pm ne peut être représenté sous forme d'une hiérarchie: la situation de communication détermine la fréquence des types de sujets et de phrases, qui eux déterminent la réalisation de la variable $\pm n e$.

Pour le traitement sociolinguistique et variationnel de la variable $\pm n e$, ceci signifie d'abord que les raisons de sa variation doivent s'envisager à travers une analyse pluridimensionnelle: c'est la combinaison des données empiriques (ici l'analyse descriptive et multifactorielle d'un corpus phonétiquement transcrit; pour d'autres analyses descriptives $c f$. Armstrong/Smith 2002, Ashby 1976, 1981, 2001, Coveney ${ }^{2} 2002$ etc.), des faits descriptifs et typologiques (comme les approches variationnelles $c f$. Koch/Oesterreicher ${ }^{2} 2011$, les analyses des clitiques dans les langues romanes, $c f$. Bossong 1998 et de la prosodie du français $c f$. Di Cristo 1998, Jun/Fougeron 2000), et finalement des tendances discursives universelles comme la PAS ( $c f$. Du Bois 1987, 2003, Lambrecht 1987) qui a permis de mieux comprendre le fonctionnement de la variable $\pm n e$ dans sa complexité.

Étant donné que \pm ne est un épiphénomène de la distribution des facteurs linguistiques qui conditionnent cette variable, il n'y a aucune raison de la concevoir comme une variable sociolinguistique en soi, comme il est pratique courante ( $c f$. Armstrong/Smith 2002: 23, Coveney 22002 : 55, Gadet 1997b). Il est également impossible que \pm ne soit en lui seul l'indicateur d'une variété ou grammaire de l'immédiat ( $c f$. Culbertson 2010, Culbertson/Legendre 2008, Massot 2010, Mensching 2008, 
Koch/Oesterreicher ${ }^{2} 2011$ et Zribi-Hertz 2011), car sa distribution dépend de la PAS, qui est une tendance discursive universelle et non une propriété du français. Finalement, la stabilité de la réalisation de ne avec les sujets lourds (même si ceux-ci sont généralement rares) indique que cette particule fait (toujours) partie du système linguistique de la majorité des francophones contemporains.

Dans le sous-chapitre 5.4, avant de conclure la discussion, nous l'avons ouverte dans différentes directions en discutant la compatibilité entre l'approche prosodique et d'autres explications de la variable $\pm n e$ et osé quelques prédictions sur le futur du ne en français moderne.

Premièrement, il semble que toutes les autres approches de la variable $\pm n e$ comportent des lignes d'argumentation qui pourraient être combinées avec celle de l'approche prosodique lors d'éventuels futurs travaux de recherche: les hypothèses externe et interne ainsi que les explications diglossique et pragmatique et même l'hypothèse du contact linguistique sont toutes, dans une certaine mesure, interprétables en termes prosodiques.

Deuxièmement, nous constatons que, malgré sa rareté dans les corpus, la particule ne continuera d'exister, également dans le français de l'immédiat. Sa réalisation dépend des facteurs linguistiques, comme les sujets lourds et les subordonnées, qui sont relativement rares dans le langage spontané, surtout en situation informelle, mais qui donnent lieu à une réalisation stable de $n e$.

Finalement, la même influence du type de sujet a déjà été prouvée par Dufter/Stark (2007) pour le début du 17e siècle. Quant au niveau diachronique, ceci signifie qu'une variation ancienne et stable de $\pm n e$ depuis plusieurs siècles est plus probable (cf. Dufter/Stark 2007, Martineau 2011, Poplack/St-Amand 2009) qu'une perte abrupte et récente de la particule (cf. Ashby 2001, Martineau/Mougeon 2003). 


\section{Conclusion}

La réalisation variable de la particule de négation ne en français est l'un des phénomènes les plus étudiés de la sociolinguistique portant sur le français moderne. Au cours de cette étude, nous avons essayé de recueillir tous les résultats existants à ce sujet, de les structurer et de les complémenter par une analyse empirique basée sur un corpus conçu explicitement à cette fin.

À l'aide de ce corpus, transcrit phonétiquement et composé de données issues de deux régions francophones et de deux situations de communication, nous avons pu montrer que la réalisation variable du proclitique négatif ne en français moderne est majoritairement déterminée par le poids phonologique du sujet grammatical de la phrase négative.

Les sujets lourds, comme les syntagmes lexicaux, les clitiques nous et vous, et les pronoms indéfinis (par exemple quelqu'un, personne), favorisent la réalisation de la particule négative, car ils offrent un contexte prosodique favorable pour un clitique dans la position préverbale. En revanche, les sujets clitique, tels que je, $t u, i l(s)$, ce et on, favorisent l'omission de ne, car ils sont en 'concurrence' prosodique avec la particule de négation.

Cette observation, basée sur une classification en termes d'un continuum morphophonologique des types de sujet, est ici implémentée au niveau théorique à l'aide du modèle prosodique de Jun/Fougeron (2000). Nous soutenons ainsi que l'omission fréquente de la particule ne avec les sujets clitiques est due, grosso-modo, à son incompatibilité avec l'accent initial secondaire du groupe accentuel, qui semble être un trait typique du français contemporain (cf. Di Cristo 1998, Hirst/Di Cristo 1996, Jun/Fougeron 2000, Vaissière 1997, Welby 2003). Plus précisément, comme les clitiques ne peuvent pas porter d'accent, l'omission de ne ainsi que d'autres stratégies de compensation prosodique interviennent afin de raccourcir la séquence d'éléments non accentogènes au début de l'énoncé et de maintenir ainsi le principe eurythmique 
(cf. Dell 1984, Di Cristo 1998: 197), c'est-à-dire l'alternation des syllabes fortes et faibles. Comme la figure (251) le montre, si un groupe accentuel comporte plus d'un proclitique, un ensemble de stratégies compensatrices intervient et permet le déplacement de l'accent initial (en gris) dans sa position naturelle (représentée par le cadre rouge) sur l'une des premières syllabes de l'énoncé. L'omission de ne est donc une stratégie qui permet la réalisation de la structure prosodique naturelle du français.

(251) L'omission de ne comme stratégie de compensation prosodique

\begin{tabular}{|c|c|c|c|c|c|c|c|}
\hline 1 & 2 & 3 & 4 & 5 & 6 & 7 & 8 \\
\hline$\sigma$ & $\sigma$ & $\sigma$ & $\sigma$ & $\sigma$ & $\sigma$ & $\sigma$ & $\sigma$ \\
\hline Je & 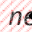 & veux & pas & & & & \\
\hline Je & 69 & le & - rends & pas & & & \\
\hline Je & no & le & ren & voie & pas & & \\
\hline Je & Fe & le & lui & ren & voie & pas & \\
\hline Je & & le & I & ra & con & te & pas \\
\hline
\end{tabular}

Comme l'illustrent les flèches en (251), l'omission de ne permet le déplacement à gauche de l'accent initial (en gris) lorsque les groupes accentuels débutent par des proclitiques.

En revanche, si un élément lourd précède la particule ne, l'accent initial peut être placé sans problèmes sur celui-ci et aucune stratégie de compensation n'est nécessaire. Par conséquent, avec les sujets lourds, $n e$ tend à être réalisé.

Nous avons vu que cette explication prosodique, illustrée en (251), est également applicable à d'autres contextes syntaxiques. Ainsi, si la variable $\pm n e$ apparaît, par exemple, dans une séquence proclitique comme dans je l'ai pas lu (1246) ou dans une construction à sujet redoublée ( $c f$. sa fille elle veut pas, 0624), la chute de la particule est probable. Par contre, si un élément lourd comme le pronom relatif qui précède ne (cf. une narratrice qui ne sert à rien, 0978), ou si ne est placé au début d'un énoncé (par exemple avec une forme verbale à l'impératif, un infinitif, etc.), sa réalisation est statistiquement probable. 
Par conséquent, même si $80 \%$ des négations dans notre corpus sont monopartites, la particule est loin de disparaître complètement en français moderne. Au contraire, dans les contextes prosodiques qui le favorisent, ne est réalisé de façon très stable par la majorité des francophones qui manifestent une variation $\pm n e$. L'analyse nous a permis de découvrir qu'il existe principalement deux ${ }^{160}$ types de locuteurs: le type $\mathrm{A}$, qui manifeste une variation $\pm n e$, et le type $\mathrm{B}$, qui montre uniquement l'absence de ne. Parmi les francophones du type $\mathrm{B}$, il y a un groupe très restreint de locutrices suisses qui, d'après nos résultats d'analyse, semblent susceptibles d'avoir éventuellement généralisé l'absence de ne indépendamment du contexte syntaxique. Néanmoins, d'autres travaux de recherche seront nécessaires pour vérifier si ces locutrices ont réellement développé une grammaire sans ne.

Une conséquence importante émerge du fait que la majorité des francophones réalise la particule ne stablement dans certains contextes syntaxiques: nous pouvons supposer que la rareté de la particule ne dans les corpus de français moderne est due à la rareté des contextes qui la favorisent et non à sa disparition générale.

De plus, dans les situations de distance communicative, l'emploi de ne est lui aussi toujours relativement répandu. Comme prévu, l'analyse a confirmé le fait bien connu que la réalisation de ne est nettement plus basse dans les conversations que pendant les examens. Par rapport à ce résultat peu surprenant, nous avons tout de même relevé un détail intéressant: les Suisses omettent la majorité des ne indépendamment de la situation de communication, tandis que les Français augmentent leur production des ne de façon significative dans une situation de distance. Néanmoins, indépendamment de la provenance des locuteurs, l'analyse multifactorielle confirme que, tout comme la rareté de ne dans les corpus en général, l'omission de ne dans les situations d'immédiat est significativement liée à la rareté des déclencheurs linguistiques dans ce type de communication.

160 Empiriquement parlant, il existe aussi un troisième type, le type $\mathrm{C}$, qui montre une présence générale de ne, mais son existence semble due aux idiosyncrasies du corpus et est donc négligeable au niveau théorique. 
Nous avons vu que les interactions entre les types de sujet et de phrase, d'un côté, et la situation de communication, de l'autre, représentent des tendances générales de notre corpus, qui sont indépendantes de la variable $\pm n e$. Nous avons pu montrer, par la suite, que c'est la situation de communication qui exerce une influence considérable sur la distribution des types de sujet et de phrase: dans l'immédiat communicatif, les sujets légers et les parataxes, qui incitent prosodiquement à l'omission de ne, sont largement préférés, tandis que, dans la distance communicative, le pourcentage des sujets lourds et des hypotaxes, qui favorisent la réalisation de ne, est élevé. Ainsi, les variables linguistiques et extralinguistiques qui influencent \pm ne peuvent être représentées sous forme de la hiérarchie en (252): la situation de communication détermine la fréquence des types de sujets et de phrases, qui, à leur tour, déterminent prosodiquement la réalisation de la variable $\pm n e$.

(252) La hiérarchie des facteurs (extra)linguistiques qui déterminent $\pm n e$

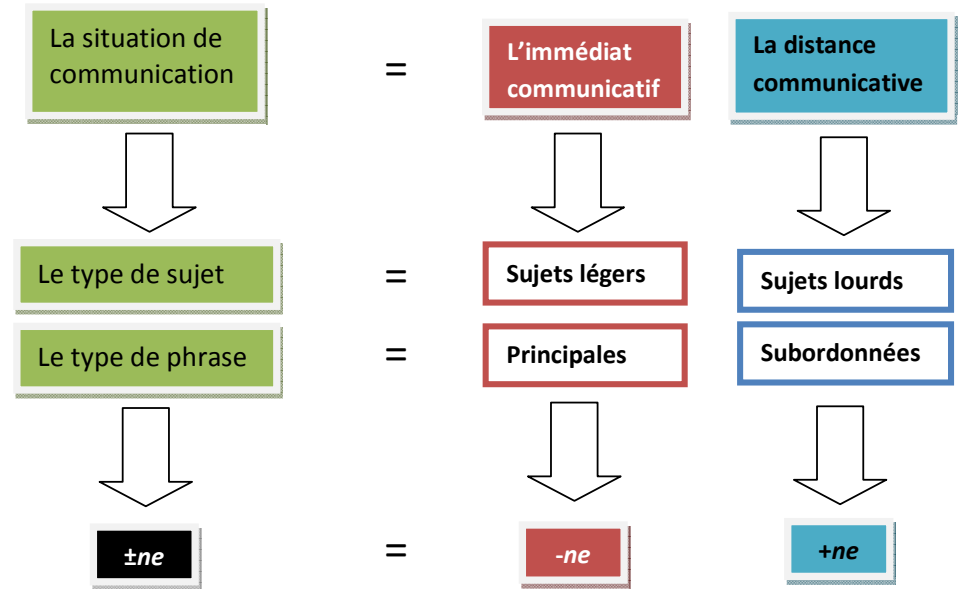

La hiérarchie en (252) montre que l'omission de ne est un épiphénomène de la distribution des facteurs linguistiques, qui, à son tour, est conditionnée par des tendances discursives universelles.

Nous constatons donc que la particule ne continuera d'exister dans le futur en français (non seulement en français standard, mais aussi 
dans le domaine de l'immédiat) malgré sa rareté dans les corpus. Sa réalisation dépend des facteurs linguistiques, comme les sujets lourds et les subordonnées, qui sont relativement peu fréquentes dans le langage spontané, surtout en situation informelle, mais qui donnent lieu à une réalisation stable de $n e$.

Nous nous permettons d'ailleurs de suggérer quelques pistes pour des futurs travaux de recherche, qui n'ont pas pu être approfondies lors du présent travail. D’un point du vue synchronique, il serait, par exemple, intéressant de combiner les explications de la variation $\pm n e$ en termes purement sociolinguistiques, syntaxiques, diglossiques ou pragmatiques avec l'explication prosodique que nous proposons ici. De plus, des analyses multifactorielles pourraient éventuellement permettre de reconsidérer d'autres variables dites 'sociolinguistiques'. Pour le français, il s'agit par de l'emploi de la liaison facultative ( $c f$. Delais-Roussarie/Durand 2003), des pronoms on vs. nous (cf. Fløttum et al. 2008) ou la construction des interrogatives ( $c f$. Coveney 22002). Finalement, l'on pourrait étudier la compétence des locuteurs qui ne réalisent aucun ne dans les corpus lors des tests d'intuition ( $c f$. Meisner 2013, Coveney 1998), afin d'établir s'ils ont vraiment développé une grammaire sans ne.

Quant au niveau diachronique, l'on pourrait explorer empiriquement l'interaction entre le début de l'omission de ne et l'évolution des proclitiques français, comme le suggère aussi Martineau (2009):

(253) The particle ne, being sandwiched between the subject affix and the verb, is deleted. If this is correct, affixation would have started at the same period as the deletion of ne, namely in Classical French. More research should be done to correlate both phenomena but as suggested in Martineau and Mougeon (2003), there are some indications that this correlation is supported by data. (Martineau 2009: 170)

Si l'on pouvait soutenir statistiquement qu'il y a une corrélation entre la cliticisation des pronoms sujet et le début de l'omission de ne à l'aide d'un corpus de français historique, cette interdépendance pourrait être formalisée comme l'intervention de deux cycles linguistiques: le cycle de l'accord sujet-verbe et le cycle négatif, comme illustré en (254). 
(254) L'interaction entre deux cycles linguistiques: la négation et l'accord

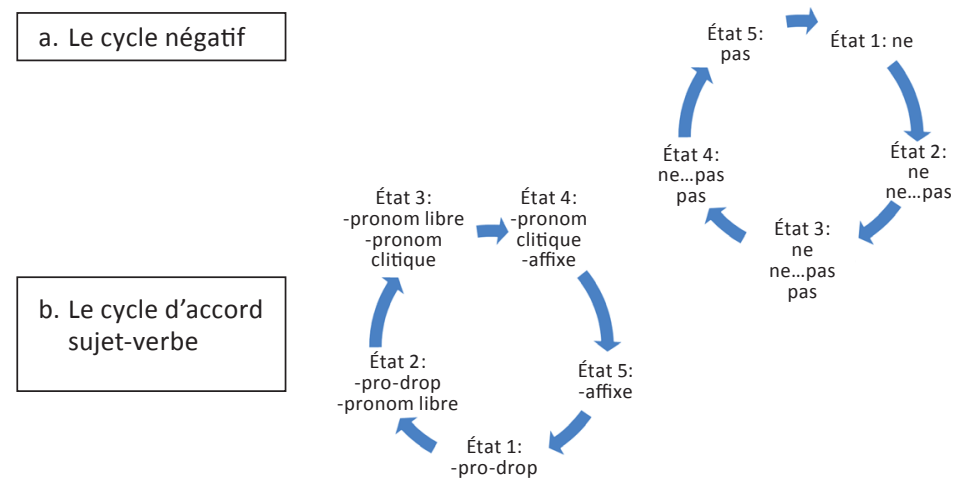

Comme la figure (254) le montre, les deux changements linguistiques pourraient être liés: le moment de la cliticisation des pronoms, donc l'état 4 du cycle d'accord sujet-verbe, pourrait intervenir entre les états 3 et 4 du cycle négatif, c'est-à-dire juste au moment où l'omission de ne devient plus fréquente.

La détermination exacte du moment où les pronoms français deviennent des clitiques obligatoires et donc prosodiquement dépendants est difficile. Il semble clair que la cliticisation des pronoms sujet a eu lieu au plus tard au moment où le Dauphin Louis XIII commence à parler (le journal d'Hérouard documente les années 1605-1610) ${ }^{161}$. Ce

161 Le latin et l'ancien français sont clairement des langues à sujet nul qui permettent habituellement l'inversion libre des sujets lexicaux et ne connaissent pas de sujets explétifs. Les pronoms personnels sujets de l'ancien français s'emploient donc surtout dans des contextes contrastifs ou de mise en relief. Ils apparaissent aussi bien en position préverbale qu'ailleurs dans la phrase. Néanmoins, déjà à partir de la deuxième moitié du $12^{\mathrm{e}}$ siècle et au début du $13^{\mathrm{e}}$ siècle, l'emploi des pronoms sujet connaît une croissance remarquable, surtout dans les subordonnées. Depuis le 13e siècle, Kaiser (1992: 154) observe l'emploi régulier des clitiques dans la langue parlée et l'emploi des sujets explétifs, ce qui est un indice de la perte des caractéristiques d'une langue à sujet nul. L'omission du sujet reste possible jusqu'aux $15^{\mathrm{e}}$ et $16^{\mathrm{e}}$ siècles et tend même à augmenter vers la fin de cette période. Marchello-Nizia (2006) estime que le pronom il est devenu clitique au $15^{\mathrm{e}}$ siècle. Roberts (2007: 35) date le changement du paramètre vers le sujet obligatoire un siècle plus tard, c'est-à-dire autour de 1600. 
journal manifeste déjà le même patron de variation $\pm n e$ en fonction du type de sujet que nous trouvons dans les corpus de français moderne (cf. Dufter/Stark 2007). À l'aide d'une analyse multifactorielle du journal d'Héroard, il serait donc éventuellement possible de trouver un lien empirique entre le cycle négatif et celui d'accord, comme nous l'avons proposé en (254).

Nous espérons que cette analyse aura contribué à une meilleure compréhension de la variation $\pm n e$ en français moderne. Nous sommes néanmoins conscientsdu fait que la présente étude ne peut être qu'une pièce dans le puzzle de la variation linguistique en français (et dans les langues naturelles en général). Nous espérons toutfois avoir montré qu'une approche pluridimensionnelle se prête à éclairer la variation $\pm n e$ en français moderne et que cette méthode pourrait être étendue à d'autres phénomènes variationnels. 
Charlotte Meisner - 978-3-0343-2332-1

Downloaded from PubFactory at 01/11/2019 02:38:59AM

via free access 


\section{Bibliographie}

Abecassis, Michaël / Ledegen, Gudrun (édd.) (2010): Les voix des Français. En parlant, en écrivant, vol. 2, Berne: Lang.

Ågren, John (1973): Étude sur quelques liaisons facultatives dans le français de la conversation radiophonique, Uppsala: Uppsala University Press.

Albizu, Cristina / Döhla, Hans-Jörg et al. (édd.) (2013): VARIANTE ET VARIÉTÉ VARIANTE E VARIETÀ - VARIANTE Y VARIEDAD VARIANTE UND VARIETÄT Actes du VIe Dies Romanicus Turicensis, Zurich, 24-25 juin 2011, Pisa: ETS.

Albrecht, Jörn / Lüdtke, Jens / Thun, Harald (édd.) (1988): Energeia und Ergon. Sprachliche Variation - Sprachgeschichte - Sprachtypologie, Tubingue: Narr.

Ammon, Ulrich / Dittmar, Norbert / Mattheier, Klaus J. (édd.) ( $\left.{ }^{2} 2004\right)$ : Sociolinguistics / Soziolinguistik. An international handbook of the science of language and society / Ein internationales Handbuch zur Wissenschaft von Sprache und Gesellschaft, Berlin/New York: de Gruyter.

Anderson, Stephen R. (1996): «How to put your clitics in their place, or why the best account of second-position may be something like the optimal one», The Linguistic Review 13, 165-191.

Anderson, Stephen R. (2000): «Towards an optimal account of second position phenomena», in: Dekkers, Joost / van der Leeuw, Frank / van de Weijer, Jeroen (édd.): Optimality theory: phonology, syntax, and acquisition, Oxford: Oxford University Press, 302-333.

Anderson, Stephen R. (2005): Aspects of the theory of clitics, New York: Oxford University Press.

Armstrong, Nigel (2001): Social and stylistic variation in spoken French. A comparative approach, Amsterdam/Philadelphie: Benjamins.

Armstrong, Nigel (2002): «Variable deletion of French ne. A cross-stylistic perspective», Language Sciences 24, 153-173.

Armstrong, Nigel / Smith, Alan (2002): «The influence of linguistic and social factors on the recent decline of French ne», Journal of French language Studies 12 , 23-41.

Arrivé, Michel / Gadet, Françoise / Galmiche, Michel (1986): La grammaire d'aujourd'hui. Guide alphabétique de linguistique française, Paris: Flammarion.

Ashby, William J. (1976): «The loss of the negative morpheme NE in Parisian French», Language 57, 647-687.

Ashby, William J. (1977) : Clitic inflection in French. An historical perspective, Amsterdam: Rodopi. 
Ashby, William J. (1981): «The loss of the negative particle ne in French: a syntactic change in progress », Language 75, 674-687.

Ashby, William J. (2001): «Un nouveau regard sur la chute du ne en français parlé tourangeau. S'agit-il d'un changement en cours?», Journal of French language Studies 11, 1-22.

Ashby, William J. / Bentivoglio, Paola (1993): «Preferred argument structure in spoken French and Spanish», Language Variation and Change 5, 61-76.

Ashby, William J. / Bentivoglio, Paola (2003): «Preferred argument structure across time and space. A comparative diachronic analysis of French and Spanish», in: Du Bois, John W. / Kumpf, Lorraine E. / Ashby, William J. (édd.): Preferred argument structure. Grammar as architecture for function, Amsterdam/Philadelphie: Benjamins, 61-80.

Astésano, Corine / Bard, Ellen (2003): «Structural and rhythmic influences on the occurrence of the initial accent in French», ICPhS 15, 503-506.

Auger, Julie (1994) : Pronominal clitics in Québec colloquial French : a morphological analysis. Thèse de doctorat, University of Pennsylvania: publiée en ligne: $<$ http:// repository.upenn.edu/cgi/viewcontent.cgi?article=1174\&context=ircs_reports $>$.

Auger, Julie / Clements, J. Clancy / Vance, Barbara (édd.) (2004) : Contemporary approaches to Romance linguistics, Amsterdam/Philadelphie: Benjamins.

Austin, Jennifer / Lawson, Jennifer (édd.) (1997): ESCOL '97. Proceedings of the Eastern States Conference on Linguistics, Ithaca: CLC Publications.

Baltin, Mark / Collins, Chris (édd.) (2000): The handbook of contemporary syntactic theory, Oxford: Blackwell.

Baronien, Luc / Martineau, France (édd.) (2009): Le français d'un continent à l'autre. Mélanges offerts à Yves Charles Morin, Sainte-Foy: pul.

Batllori, Montse / Hernandez, Maria Luïsa / Roca, Francesc (édd.) (2005): Grammaticalization and parametric variation, New York: Oxford University Press.

Battye, Adrian / Roberts, Ian (édd.) (1995): Clause structure and language change, Oxford: Oxford University Press.

Bauche, Henri (1951): Le langage populaire. Grammaire, syntaxe et dictionnaire du français tel qu'on le parle dans le peuple avec tous les termes d'argot usuel, Paris: Payot.

Baude, Olivier (2006): Corpus oraux. Guide des bonnes pratiques, Paris: CNRS.

Baude, Olivier (2007): «Aspects juridiques et éthiques de la conservation et de la diffusion des corpus oraux", Revue française de linguistique appliquée 12, 85-79.

Bedijs, Kristina (2012): Die inszenierte Jugendsprache. Von «Ciao, amigo!» bis «Wesh, tranquille!». Entwicklungen der französischen Jugendsprache in Spielfilmen (1958-2005), Munich: Meidenbauer.

Beeching, Kate / Armstrong, Nigel / Gadet, Françoise (édd.) (2009): Sociolinguistic variation in contemporary French, Amsterdam/Philadelphie: Benjamins.

Bell, Alan (1984): «Language style as audience design», Language in Society 13, 145-204. 
Bell, Alan (2001): «Back in style», Eckert, Patricia / Rickford, John R. (édd.): Style and sociolinguistic variation, New York: Cambridge University Press, 139-169.

Belletti, Adriana (1999): «Italian / Romance clitics: structure and derivation», in: van Riemsdijk, Henk (éd.): Clitics in the languages of Europe, Berlin: Mouton de Gruyter, 543-579.

Benincà, Paola (1991): "Complement clitics in medieval Romance: The ToblerMussafia law », in: van Riemsdijk, Henk / Rizzi, Luigi (édd.): Clitics and their hosts. ESF-Eurotype report. Theme group 8: clitics, Tilburg: Department of Language and Literature Tilburg University, 1-27.

Benincà, Paola (1995): «Complement clitics and the Tobler-Mussafia law», in: Battye, Adrian / Roberts, Ian (édd.): Clause structure and language change, Oxford: Oxford University Press, 325-344.

Benkirane, Thami (1998): «Intonation in western Arabic (Morocco)», in: Hirst, Daniel/ Di Cristo, Albert (édd.): Intonation systems. A survey of twenty languages, Cambridge: Cambridge University Press, 345-359.

Bernini, Giuliano / Ramat, Paolo (1996): Negative sentences in the languages of Europe: a typological approach, Berlin/New York: Mouton de Gruyter.

Biberauer, Theresa / Roberts, Ian (2011): «Negative words and related expressions. A new perspective on some familiar puzzles », in: Larrivée, Pierre / Ingham, Richard P. (édd.): The evolution of negation. Beyond the Jespersen Cycle, 23-60.

Bilger, Mireille (éd.) (2000): Corpus. Méthodologie et applications linguistiques, Paris: Champion.

Bilger, Mireille (2002): "Corpus de français parlé. Recueil et analyse», in: Pusch, Claus D. / Raible, Wolfgang (édd.): Romanistische Korpuslinguistik - Korpora und gesprochene Sprache / Romance corpus linguistics - corpora and spoken language, Tubingue: Narr, 45-58.

Bilger, Mireille (2008): Données orales. Les enjeux de la transcription, Perpignan: PUP.

Blanche-Benveniste, Claire / Jeanjean, Colette (1987): Le français parlé. Transcription et édition, Paris: Didier Erudition.

Blanche-Benveniste, Claire / Rouget, Christine / Sabio Frédéric (édd.) (2002): Choix de textes de français parlé. 36 extraits, Paris: Champion.

Blancquaert, Edgard (1923): «Over de dubbele ontkenning en nog wat», Handelingen van het Vlaamse filologencongres 6, 60-69.

Bossong, Georg (1998): «Vers une typologie des incidences actancielles. Les clitiques romans dans une perspective comparative», in: Ramat, Paolo / Roma, Elisa (édd.): Sintassi Storica. Atti del congresso internazionale della società di linguistica italiana, Pavia, 26-28 settembre 1996, Rome: Bulzoni, 9-43.

Botinis, Antonis (éd.) (2000): Intonation: Analysis, modeling and technology, Dordrecht: Kluwer.

Breitbarth, Anne (2009): «A hybrid approach to Jespersen's cycle in West Germanic », Journal of comparative Germanic linguistics 12.2, 81-114. 
Breitbarth, Anne / Haegeman, Liliane (à paraître): En en is niet wat we dachten: A Flemish discourse particle, Paper presented in April 2011 at MOSS 2, Institute of Russian Language of the Russian Academy of Sciences, Moscow.

Breitbarth, Anne / Haegeman, Liliane (2010): «Continuity is change: the long tail of Jespersen's cycle in Flemish", in: Breitbarth, Anne / Lucas, Christopher et al. (édd.): Continuity and change in grammar, Amsterdam: Benjamins, 61-76.

Breitbarth, Anne / Lucas, Christopher et al. (édd.) (2010): Continuity and change in grammar, Amsterdam: Benjamins.

Brunot, Ferdinand / Bruneau, Charles (1949): Précis de grammaire historique de la langue française, Paris: Masson.

Brustad, Kristen E. (2000): Syntax of spoken Arabic. A comparative study of Moroccan, Egyptian, Syrian, and Kuwaiti Dialects, Washington: Georgetown University Press.

Busse, Winfried / Dubost, Jean-Pierre 2(1983): Französisches Verblexikon: die Konstruktion der Verben im Französischen, Stuttgart: Klett.

Bußmann, Hadumod (éd.) ( $\left.{ }^{4} 2008\right)$ : Lexikon der Sprachwissenschaft, Stuttgart: Kröner.

Bybee, Joan L. (1985): Morphology. A study of the relation between meaning and form, Amsterdam/Philadelphie: Benjamins.

Cappeau, Paul / Gadet, Françoise (2007): «Où en sont les corpus sur les français parlés?», in: Revue française de linguistique appliquée 12, 129-133.

Cardinaletti, Anna / Starke Michal (1999): «The typology of structural deficiency. A case study of the three classes of pronouns», in: van Riemsdijk, Henk (éd.): Clitics in the languages of Europe, Berlin: Mouton de Gruyter, 146-233.

Carvalho, Paulo de / Muller, Claude et al. (édd.) (2001): Clitiques et cliticisation. Actes du colloques de Bordeaux, octobre 1998, Paris: Champion.

Cheshire, Jenny / Stein, Dieter (édd.) (1997): Taming the Vernacular. From dialect to written standard, London/New York: Longman.

Cheshire, Jenny / Stein Dieter (1997): «The syntax of spoken language», in: Cheshire, Jenny / Stein Dieter (éd.): Taming the vernacular. From dialect to written standard, London/New York: Longman, 1-12.

Chomsky, Noam (1995): The minimalist program, Cambridge: MIT Press.

Chomsky, Noam (2000): «Minimalist inquiries: the framework», in: Martin, Roger / Michaels, David / Uriagereka, Juan (édd.): Step by step. Essays on minimalist syntax in honor of Howard Lasnik, Cambridge, Mass. : MIT Press, 89-156.

Chomsky, Noam (2001): «Derivation by phase», in: Kenstowicz, Michael J. (éd.): Ken Hale: a life in language, Cambridge, Mass. : MIT Press, 1-52.

Cinque, Guglielmo / Koster, Jan et al. (édd.) (1994): Paths towards Universal Grammar. Studies in honor of Richard S. Kayne Washington D.C.: Georgetown University Press.

Cole, Peter (éd.) (1978): Pragmatics, Orlando: Academic Press.

Combettes Bernard, Marchello-Nizia Christiane (édd.) (2007): Etudes sur le changement linguistique en français, Nancy: Presses Universitaires de Nancy. 
Corblin, Francis / de Swart, Henriette (édd.) (2004): Handbook of French semantics, Stanford: CSLI.

Coseriu, Eugenio (1988a): «Die Begriffe 'Dialekt', 'Niveau' und 'Sprachstil' und der eigentliche Sinn der Dialektologie [1981]», in: Albrecht, Jörn / Lüdtke, Jens / Thun, Harald (édd.): Energeia und Ergon. Sprachliche Variation - Sprachgeschichte-Sprachtypologie, Tubingue: Narr, 15-43.

Coseriu, Eugenio (1988b) : “'Historische Sprache' und 'Dialekt' [1981]», in: Albrecht, Jörn / Lüdtke, Jens / Thun, Harald (édd.): Energeia und Ergon. Sprachliche Variation - Sprachgeschichte - Sprachtypologie, Tubingue : Narr, 45-61.

Costa, João (éd.) (2000): Portuguese syntax: new comparative studies, New York/ Oxford: Oxford University Press.

Coupland, Nicolas (2007): Style. Language variation and identity, Cambridge, UK: Cambridge University Press.

Coveney, Aidan (1998): "Awareness of linguistic constraints on variable ne omission », Journal of French Language Studies 8, 159-187.

Coveney, Aidan (2010): "Vouvoiement and tutoiement: sociolinguistic reflections », Journal of French Language Studies 20, 127-150.

Coveney, Aidan (2011): «A language divided against itself? Diglossia, code-switching and variation in French», in: Martineau, France / Nadasdi, Terry J. (édd.) : Le français en contact: hommages à Raymond Mougeon, Québec: Presses de l'Université Laval, 51-85.

Coveney, Aiden (2002): Variability in spoken French. A sociolinguistic study of interrogation and negation, Bristol, UK/ Portland, USA : Elm Bank.

Cowan, David (1958): An introduction to modern literary Arabic: Cambridge University Press.

Cresti, Emanuela / Moneglia, Massimo (2005): C-Oral-Rom. Integrated reference corpora for spoken Romance languages, Amsterdam/Philadelphie: Benjamins.

Crouzet, Olivier / Angoujard, Jean-Pierre (édd.) (2007): Actes des / Proceedings of JEL 2007 Nantes: Université de Nantes.

Crystal, David ( $\left.{ }^{3} 1991\right)$ : A Dictionary of Linguistics and Phonetics, Blackwell: Oxford.

Culbertson, Jennifer (2010): «Convergent evidence for categorical change in French: from subject clitic to agreement marker», Language 86, 85-132.

Culbertson, Jennifer / Legendre, Géraldine (2008): «Qu'en est-il des clitiques sujet en français oral contemporain?», in: Durand, Jacques / Habert, Benoît / Laks, Bernard (édd.): Actes du ler Congrès Mondial de Linguistique française, Paris : EDP Sciences, 2651-2662.

Dahl, Östen (1979): «Typology of sentence negation», Linguistics 17, 79-106.

Dahl, Östen (2010) : «Typology of negation», in: Horn, Laurence R. (éd.): The expression of negation, Berlin: Mouton de Gruyter, 9-40.

Damourette, Jacques / Pichon, Edouard (1911-1927): Des mots à la pensée. Essai de grammaire de la langue française, Paris: La Rochefoucauld. 
De Cat, Cécile (2007): French dislocation. Interpretation. Syntax. Acquisition, Oxford: Oxford University Press.

Déchaine, Rose-Marie / Wiltschko, Martina (2002): «Decomposing pronouns», Linguistic Inquiry 33, 409-442.

Dekkers, Joost / van der Leeuw, Frank / van de Weijer, Jeroen (édd.) (2000): Optimality theory: phonology, syntax, and acquisition, Oxford: Oxford University Press.

Delais-Roussarie, Elisabeth / Durand, Jacques (2003): Corpus et variation en phonologie du français. Méthodes et analyses, Toulouse: Presses Universitaires du Mirail.

Delattre, Pierre (1940): «Le mot est-il une entité phonétique en français?», Le Français Moderne 8, 47-56.

Dell, François (1984): «L'accuentation dans les phrases en français», in: Dell, François / Hirst, Daniel / Vergnaud, Jean-Roger (édd.): Forme sonore du langage. Structure des représentations en phonologie, Paris: Hermann, 65-122.

Dell, François / Hirst, Daniel / Vergnaud, Jean-Roger (édd.) (1984): Forme sonore du langage. Structure des représentations en phonologie, Paris: Hermann.

Déprez, Viviane (2003) : «Concordance négative, syntaxe des mots-N et variation dialectale», Cahiers de linguistique francaise 25, 97-118.

Déprez, Viviane / Martineau, France (2004) : «Micro-parametric variation and negative concord», in: Auger, Julie / Clements, J. Clancy / Vance, Barbara (édd.) : Contemporary approaches to Romance linguistics, Amsterdam/Philadelphie: Benjamins, 139-158.

Désirat, Claude / Hordé, Tristan (1976): La langue française au $20^{e}$ siècle, Paris: Bordas.

Di Cristo, Albert (1998): «Intonation in French», in: Hirst, Daniel / Di Cristo, Albert (édd.): Intonation systems. A survey of twenty languages, Cambridge: Cambridge University Press, 195-218.

Diller, Anne-Marie (1983): «Subject NP Structure and variable constraints: the case of French NE deletion», in: Fasold, Ralph W. (éd.): Variation in the form and the use of language, Washington: Georgetown University Press.

Dittmar, Norbert (1997): Grundlagen der Soziolinguistik: ein Arbeitsbuch mit Aufgaben, Tubingue: Niemeyer.

Dryer, Matthew S. (2011a): «Chapter 112. Negative Morphemes», in: Haspelmath, Martin / Dryer, Matthew S. et al. (édd.): The world atlas of language structures online, Munich: Max Planck Digital Library.

Dryer, Matthew S. (2011b): «Chapter 143. Order of Negative Morpheme and Verb», in: Haspelmath, Martin / Dryer, Matthew S. et al. (édd.): The world atlas of language structures online, Munich: Max Planck Digital Library.

Du Bois, John W. (1987): "The discourse basis of ergativity», Language 63, 805-855.

Du Bois, John W. (2003): «Argument structure. Grammar in use», in: Du Bois, John W. / Kumpf, Lorraine E. / Ashby, William J. (édd.) : Preferred argument structure. Grammar as architecture for function, Amsterdam/Philadelphie: Benjamins, 11-60. 
Du Bois, John W. / Kumpf, Lorraine E. / Ashby, William J. (édd.) (2003): Preferred argument structure. Grammar as architecture for function, Amsterdam/Philadelphie: Benjamins.

Dubois, Jean (1967): Grammaire structurale du français. Le verbe, Paris: Larousse.

Ducrot, Oswald (1972): Dire et ne pas dire, Paris: Herman.

Dufter, Andreas (2007): «Clefting and discourse organization: comparing Germanic and Romance», in: Dufter, Andreas / Jacob, Daniel (édd.): Focus and background in the Romance languages, Amsterdam/Philadelphie: Benjamins, 83122.

Dufter, Andreas (2013): «Sur l'absence du ne de négation dans le français du dixneuvième siècle», in: Grutschus, Anke / Fesenmeier, Ludwig / Patzelt, Carolin (édd.): Absences(s) - Phänomene sprachlicher Absenz und Möglichkeiten ihrer Analyse, Francfort s.-1.-M. : Klostermann.

Dufter, Andreas / Jacob, Daniel (édd.) (2007): Focus and Background in the Romance Languages, Amsterdam/Philadelphie: Benjamins.

Dufter, Andreas / Stark, Elisabeth (2002): «La variété des variétés. Combien de dimensions pour la description? Quelques réflexions à partir du français», Romanistisches Jahrbuch 53, 81-108.

Dufter, Andreas / Stark, Elisabeth (2007): «La linguistique variationnelle et les changements linguistiques 'mal compris'. Le cas de la 'disparition' du ne de négation », in: Combettes, Bernard / Marchello-Nizia, Christiane (édd.): Etudes sur le changement linguistique en français, Nancy: Presses Universitaires de Nancy, 115-128.

Durand, Jacques / Habert, Benoît / Laks, Bernard (édd.) (2008): Actes du ler Congrès Mondial de Linguistique française, Paris: EDP Sciences.

Durand, Jacques / Laks, Bernard / Lyche, Chantal (2002): «La phonologie du français contemporain: usages, variétés et structure», in: Pusch, Claus D. / Raible, Wolfgang (édd.): Romanistische Korpuslinguistik - Korpora und gesprochene Sprache / Romance corpus linguistics - corpora and spoken language, Tubingue: Narr, 93-106.

Durand, Jacques / Laks, Bernard / Lyche, Chantal (2005) : «La phonologie du français contemporain: usages, variétés et structure.», in: Pusch, Claus D. / Kabatek, Johannes / Raible, Wolfgang (édd.): Korpora und diachrone Sprachwissenschaft II. Corpora and diachronic linguistics II, Tubingue: Narr, 93-106.

Durand, Jacques / Laks, Bernard / Lyche, Chantal (édd.) (2009): Phonologie, variation et accents du français, Paris: Hermès.

Durand, Jacques / Laks, Bernard / Lyche, Chantal (2009): «Le projet PFC: une source de données primaires structurées», in: Durand, Jacques / Laks, Bernard / Lyche, Chantal (édd.): Phonologie, variation et accents du français, Paris: Hermès, 19-61.

Durand, Jacques / Kawaguchi, Yuji / Minegishi, Makoto (édd.) (2009) : Corpus analysis and variation in linguistics, Amsterdam/Philadelphie: Benjamins. 
Dworkin, Steve / Wanner, Dieter (édd.) (2000): New approaches to old problems. Issues in historical linguistics, Amsterdam/Philadelphie: Benjamins.

Eckardt, Regine (2003): Eine Runde im Jespersen-Zyklus, emphatische Negation und negativ-polare Elemente im Altfranzösischen, publié en ligne: <http://nbnresolving.de/urn:nbn:de:bsz: 352-opus-9910>.

Eckert, Patricia / Rickford, John R. (édd.) (2001): Style and sociolinguistic variation, New York: Cambridge University Press.

Ernst, Gerhard (1985): Gesprochenes Französisch zu Beginn des 17. Jahrhunderts. Direkte Rede in Jean Héroards "Histoire particulière de Louis XIII» (16051610), Tubingue: Niemeyer.

Ernst, Gerhard / Glessgen, Martin-Dietrich et al. (édd.) (2006): Romanische Sprachgeschichte: Ein internationales Handbuch zur Geschichte der romanischen Sprachen, Berlin/New York: de Gruyter.

Fasold, Ralph W. (éd.) (1983): Variation in the form and the use of language, Washington: Georgetown University Press.

Fauconnier, Gilles (1975): «Polarity and the Scale Principle», Papers from the 11th Regional Meeting of the Chicago Linguistic Society, 188-199.

Ferguson, Charles A. (1959): «Diglossia», Word 15, 324-340.

Finegan, Edward / Biber, Douglas (2001) : «Register variation and social dialect variation: the Register Axiom», in: Eckert, Patricia / Rickford, John R. (édd.): Style and sociolinguistic variation, New York: Cambridge University Press, 235-267.

Fløttum, Kjersti / Jonasson, Kerstin / Norén, Coco (2008): On - pronom à facettes, Paris: Duculot.

Flydal, Leiv (1951): «Remarques sur certains rapports entre le style et l'état de langue», Norsk Tidsskrift for Sprogvidenskap 16, 240-257.

Fodor, Jerry A. / Katz, Jerrold J. (édd.) (1964): The structure of language. Readings in the philosophy of language, Englewood Cliffs: NJ : Prentice-Hall.

Fónagy, Ivan (1979) : «L'accent français : accent probabilitaire», Studia Phonetica 15, 123-133.

Fonseca-Greber, Barbara / Waugh, Laura (2002): «The subject clitics of conversational European French: morphologization, grammatical change, semantic change, and change in progress», in: Núñez-Cedeño, Rafael / López, Luis / Cameron, Richard (édd.): A Romance perspective on language knowledge and use, Amsterdam/Philadelphie: Benjamins, 99-117.

Fonseca-Greber, Bonnibeth B. (2007): "The emergence of emphatic ne in conversational Swiss French», Journal of French language Studies 17, 249-275.

Forner, Werner (2006): «Prinzipien der Funktionalstilistik», in: Ernst, Gerhard / Glessgen, Martin-Dietrich et al. (édd.): Romanische Sprachgeschichte: Ein internationales Handbuch zur Geschichte der romanischen Sprachen, Berlin/ New York: de Gruyter, 1907-1923.

Fougeron, Cécile / Jun, Sun-Ah (1998): «Rate effects on French intonation: prosodic organization and phonetic realization», Journal of Phonetics 26, 45-69. 
Frei, Henri (1929): La grammaire des fautes, Paris: Librairie Paul Geuthner.

Gaatone, David (1971): Etude descriptive du système de la négation en français contemporain, Genève: Droz.

Gadet, Françoise ( $\left.{ }^{2} 1997 \mathrm{a}\right)$ : Le français ordinaire, Paris : Colin.

Gadet, Françoise (1997b) : «La variation, plus qu'une écume», Langue Française 115, 5-29.

Gadet, Françoise (2000): «Des corpus pour (ne)...pas», in: Bilger, Mireille (éd.): Corpus. Méthodologie et applications linguistiques, Paris: Champion, 156-167.

Gadet, Françoise (2004): «Research on sociolinguistic style. Soziolinguistische Stilforschung», in: Ammon, Ulrich / Dittmar, Norbert / Mattheier, Klaus J. (édd.) : Sociolinguistics / Soziolinguistik. An international handbook of the science of language and society / Ein internationales Handbuch zur Wissenschaft von Sprache und Gesellschaft, Berlin/New York: de Gruyter, 1353-1361.

Gadet, Françoise (2007): La variation sociale en français, Paris: Ophrys.

Garde, Paul (1968): L'accent, Paris: Presses Universitaires de France.

Gardiner, Alan H. (1904): «The word \#\#\#\#», Zeitschrift für Ägyptische Sprache und Altertumskunde 41, 130-135.

Gerstenberg, Annette (2011): Generation und Sprachprofile im höheren Lebensalter. Untersuchungen zum Französischen auf der Basis eines Korpus biographischer Interviews, Francfort s.-1.-M.: Klostermann.

Gess, Randall / Arteaga, Deborah (édd.) (2006): Historical Romance linguistics: retrospective and perspectives, Amsterdam/Philadelphie: Benjamins.

Gibbon, Dafydd (1998): «Intonation in German», in: Hirst, Daniel / Di Cristo, Albert (édd.): Intonation systems. A survey of twenty languages, Cambridge: Cambridge University Press, 78-95.

Givón, Talmy (1976): «Topic, pronoun and grammatical agreement», in: Li, Charles N. (éd.): Subject and topic, New York: Academic Press, 149-188.

Givón, Talmy (1978): «Negation in language: pragmatics, function, ontology», in: Cole, Peter (éd.): Pragmatics, Orlando: Academic Press, 69-112.

Godard, Danièle (éd.) (2003): Les langues romanes: problèmes de la phrase simple, Paris: CNRS.

Grevisse, Maurice / Goosse, André (152011): Le bon usage. Grammaire française, Bruxelles: De Boeck \& Larcier.

Gries, Stefan T. (2009): Quantitative corpus linguistics with R. A practical introduction, New York: Routledge.

Grieve-Smith, Angus (2010): «Modeling the semantic and pragmatic evolution of ne ...pas in French», in: Neveu, Frank / Muni Toke, Valelia et al. (édd.): Congrès Mondial de Linguistique Française - CMLF 2010, Paris: Institut de Linguistique Française, 223-236.

Grutschus, Anke/Fesenmeier, Ludwig/Patzelt, Carolin(édd.) (2013): Absences(s) - Phänomene sprachlicher Absenz und Möglichkeiten ihrer Analyse, Francfort s.-1.-M.: Klostermann. 
Haegeman, Liliane (21996): The syntax of negation, Cambridge: Cambridge University Press.

Haegeman, Liliane (éd.) (1997): Elements of grammar: a handbook of generative syntax, Dordrecht: Kluwer.

Haegeman, Liliane (2002): «West Flemish negation and the derivation of SOV order in West Germanic», Nordic Journal of Linguistics 25, 154-189.

Hansen, Anita Berit / Malderez, Isabelle (2004): «Le ne de négation en région parisienne. Une étude en temps réel», Langage et société 107, 5-30.

Harrell, Richard S. (1962): A short reference grammar of Moroccan Arabic, Washington: Georgetown University Press.

Haspelmath, Martin (1997): Indefinite pronouns, Oxford: Claredon Press.

Haspelmath, Martin / Dryer, Matthew S. et al. (édd.) (2011): The world atlas of language structures online, Munich: Max Planck Digital Library (publié en ligne: $<$ http://wals.info/>.

Heap, David (2000): La variation grammaticale en géolinguistique. Les pronoms sujet en roman central. Toronto/Munich: LINCOM Europa.

Hirschbühler, Paul / Labelle, Marie (2000): «Evolving Tobler-Mussafia effects in the placement of French clitics», in: Dworkin, Steve / Wanner, Dieter (édd.): New approaches to old problems. Issues in historical linguistics, Amsterdam/Philadelphie: Benjamins, 165-182.

Hirschbühler, Paul / Labelle, Marie (2001): «La position des clitiques par rapport au verbe à l'impératif dans l'évolution du français», Recherches Linguistiques 30.13, 13-38.

Hirschbühler, Paul / Labelle, Marie (2003): «Residual Tobler-Mussafia effects in French dialects », in: Pérez, Ana T. / Roberge, Yves (édd.) : Romance linguistics : theory and acquisition, Amsterdam/Philadelphie: Benjamins, 149-164.

Hirschbühler, Paul / Labelle, Marie (2006): «Proclisis and enclisis of object pronouns at the turn of the 17th century», in: Gess, Randall / Arteaga, Deborah (édd.): Historical Romance Linguistics: retrospective and perspectives, Amsterdam/ Philadelphie: Benjamins, 187-208.

Hirst, Daniel (1998): «Intonation in British English», in: Hirst, Daniel / Di Cristo, Albert (édd.): Intonation systems. A survey of twenty languages, Cambridge: Cambridge University Press, 56-77.

Hirst, Daniel / Di Cristo, Albert (1996): «Y a-t-il des unités tonales en français?», JEP 21, 223-226.

Hirst, Daniel / Di Cristo, Albert (édd.) (1998): Intonation systems. A survey of twenty languages, Cambridge: Cambridge University Press.

Hoeksema, Jack (2000) : «Negative Polarity Items : triggering, scope, and c-command», in: Horn, Laurence / Kato, Yasuhiko (édd.): Negation and polarity. Syntactic and semantic perspectives, Oxford/New York: Oxford University Press, 115-146.

Holtus, Günther / Metzeltin, Michael / Schmitt, Christian (édd.) (2001): Lexikon der Romanistischen Linguistik (LRL), vol. 2.1, Tubingue: Niemeyer. 
Horn, Laurence R (22001): A natural history of negation, Stanford, Calif.: CSLI.

Horn, Laurence R. (éd.) (2010): The expression of negation, Berlin/New York: Mouton de Gruyter.

Horn, Laurence R. (2010): «Introduction», in: Horn, Laurence R. (éd.): The expression of negation, Berlin/New York: Mouton de Gruyter, 1-7.

Horn, Laurence R. / Kato, Yasuhiko (édd.) (2000): Negation and polarity. Syntactic and semantic perspectives, Oxford/New York: Oxford University Press.

Horn, Laurence R. / Kato, Yasuhiko (2000): «Introduction. Negation and polarity at the millenium », in: Horn, Laurence R. / Kato, Yasuhiko (édd.): Negation and polarity. Syntactic and semantic perspectives, Oxford: Oxford University Press, $1-19$.

Jaeggli, Osvaldo (1982): Topics in Romance syntax, Dordrecht: Foris.

Jäger, Agnes (2008): History of german negation, Amsterdam: John Benjamins.

Jespersen, Otto (1917): Negation in English and other languages, København: Hoest. Jespersen, Otto (1924): The philosophy of grammar, London: Allen \& Unwin et al. Jespersen, Otto (1992 [1924]): La philosophie de la grammaire Paris: Gallimard.

Jones, Michael A. ${ }^{3}(2007)$ : Foundations of French syntax, Cambridge: Cambridge University Press.

Jun, Sun-Ah / Fougeron, Cécile (1995): «The accentual phrase and the prosodic structure of French », Proceedings of the International Congress of Phonetic Sciences $13,722-725$.

Jun, Sun-Ah / Fougeron, Cécile (2000): «A phonological model of French intonation», in: Botinis, Antonis (éd.): Intonation: analysis, modeling and technology, Dordrecht: Kluwer, 209-242.

Jun, Sun-Ah / Fougeron, Cécile (2002): «Realizations of accentual phrase in French», Probus 14, 147-172.

Kaiser, Georg A. (1992): Die klitischen Personalpronomina im Französischen und Portugiesischen. Eine synchronische und diachronische Analyse, Francfort s.-1.-M: Vervuert.

Kaiser, Georg A. (2008): «Zur Grammatikalisierung der französischen Personalpronomina», in: Stark, Elisabeth / Schmidt-Riese, Roland / Stoll, Eva (édd.) : Romanische Syntax im Wandel, Tubingue: Narr, 305-326.

Kawaguchi, Yuji (2009): «Les particules négatives du français : ne, pas, point et mieun aperçu historique», in: Baronien, Luc / Martineau, France (édd.): Le français d'un continent à l'autre. Mélanges offerts à Yves Charles Morin, Sainte-Foy: pul, 193-210.

Kayne, Richard S. (1975): French syntax. The transformational cycle, Cambridge, Mass. : MIT-Press.

Kayne, Richard S. (1991): «Romance clitics, verb movement, and PRO», Linguistic Inquiry 22, 647-686.

Kenstowicz, Michael J. (éd.) (2001): Ken Hale: a life in language, Cambridge, Mass. : MIT Press. 
Klima, Edward (1964): «Negation in English», in: Fodor, Jerry A. / Katz, Jerrold J. (édd.): The structure of language. Readings in the philosophy of language, Englewood Cliffs, NJ: Prentice-Hall, 246-323.

Knüppel, Veronika (2001): Die Syntax der Negation im Französischen. Eine lexikalisch-funktionale Analyse, Tubingue: Niemeyer.

Koch, Peter / Oesterreicher, Wulf (2001): «Gesprochene Sprache und geschriebene Sprache / Langage parlé et langage écrit», in: Holtus, Günther / Metzeltin, Michael / Schmitt, Christian (édd.): Lexikon der Romanistischen Linguistik (LRL), vol. 2.1, Tubingue: Niemeyer, 584-637.

Koch, Peter / Oesterreicher, Wulf (2011): Gesprochene Sprache in der Romania. Französisch, Italienisch, Spanisch, Berlin/New York: de Gruyter.

Krifka, Manfred (1995): «The semantics and pragmatics of polarity items», Linguistic analysis $25,209-257$.

Kroch, Anthony (2000): «Syntactic change», in: Baltin, Mark / Collins, Chris (édd.): The handbook of contemporary syntactic theory, Oxford: Blackwell.

Labelle, Guy (1976): La performance syntaxique d'enfants de 5 ans de Montréal et de Paris. Thèse de doctorat inédite, Paris: Université de Paris VII.

Labelle, Marie / Hirschbühler, Paul (2001): «Les clitiques arguments en serbo-croate et dans l'histoire du français», in: Carvalho, Paulo de / Muller, Claude et al. (édd.): Clitiques et cliticisation. Actes du colloques de Bordeaux, octobre 1998, Paris: Champion, 109-132.

Labov, William (1972a): Language in the Inner City, Philadelphie: University of Pennsylvania Press.

Labov, William (1972b): Sociolinguistic Patterns, Oxford: Blackwell.

Labov, William (2001): «The anatomy of style-shifting», in: Eckert, Patricia / Rickford, John R. (édd.): Style and sociolinguistic variation, New York: Cambridge University Press, 85-108.

Ladusaw, William A. (1996): «Negation and Polarity Items», in: Lappin, Shalom (éd.): The handbook of contemporary semantic theory, Oxford: Blackwell, 321-341.

Laka, Itziar (1990): Negation in syntax: on the nature of functional categories and projections. Thèse de doctorat, Boston, MA: MIT.

Lambrecht, Knud (1987): «On the status of SVO sentence in French discourse», in: Tomlin, Russel S. (éd.): Coherence and grounding in discourse, Amsterdam: Benjamins.

Lang, Margaret / Perez, Isabelle (1996): Modern French grammar. A practical guide, New York: Routledge.

Lappin, Shalom (éd.) (1996): The handbook of contemporary semantic theory, Oxford: Blackwell.

Larrivée, Pierre (2014): «Reanalysis of negatives as polarity markers? The last 400 years of decline of the French preverbal negative clitic», in: Stark, Elisabeth / Meisner, Charlotte / Völker, Harald (édd.): Jespersen revisited: Negation in Romance and beyond (= Special issue, Lingua). 
Larrivée, Pierre (2004): L'association négative. Depuis la syntaxe jusqu'à l'interprétation, Genève: Droz.

Larrivée, Pierre / Ingham, Richard P. (édd.) (2011): The evolution of negation. Beyond the Jespersen Cycle, Berlin/New York: de Gruyter.

Le Bidois, Georges / Le Bidois, Robert (1938): Syntaxe du français moderne. Ses fondements historiques et psychologiques, vol. 2, Paris: Picard.

Legendre, Géraldine (1996): Clitics, verb (non-)movement, and optimality in Bulgarian. Technical report. JHU-CogSci-96-5, Baltimore: Johns Hopkins University Press.

Legendre, Géraldine (1997): «Second position clitics in a verb-second language: conflict resolution in Macedonian», in: Austin, Jennifer / Lawson, Jennifer (édd.): ESCOL '97. Proceedings of the Eastern States Conference on Linguistics, Ithaca: CLC Publications, 139-149.

Legendre, Géraldine (2000): «Morphological and prosodic alignment of Bulgarian clitics», in: Dekkers, Joost / van der Leeuw, Frank / van de Weijer, Jeroen (édd.): Optimality theory: phonology, syntax, and acquisition, Oxford: Oxford University Press, 423-462.

Lemieux, Monique (1985): «'Pas rien'», in: Lemieux, Monique / Cedergren, Henrietta J. (édd.): Les tendances dynamiques du français parlé à Montréal, Québec: Office de la langue française, 91-139.

Lemieux, Monique / Cedergren, Henrietta J. (édd.) (1985): Les tendances dynamiques du français parlé à Montréal, Québec: Office de la langue française.

Li, Charles N. (éd.) (1976): Subject and topic, New York: Academic Press.

Lightfoot, David (1999): The development of language: acquisition, change, and evolution Malden, MA: Blackwell.

Lindblom, Björn (1990): «Explaining phonetic variation: a sketch of the $\mathrm{H}$ and $\mathrm{H}$ theory», in: Marchal, Alain / Hardcastle, William J. (édd.): Speech production and speech modelling, Dordrecht: Kluwer, 403-439.

Lüdicke, Annemarie (1982): «Zum Ausfall der Verneinungspartikel ne im gesprochenen Französisch», Zeitschrift für Romanische Philologie 98, 43-58.

Ludwig, Ralph (1988): Korpus. Texte des gesprochenen Französisch. Materialien I, Tubingue: Narr.

Marchal, Alain / Hardcastle, William J. (édd.) (1990): Speech production and speech modelling, Dordrecht: Kluwer.

Marchello-Nizia, Christiane (1997): La langue française aux XIVe et XVe siècles, Paris: Nathan.

Martin, Roger / Michaels, David / Uriagereka, Juan (édd.) (2000) : Step by step. Essays on minimalist syntax in honor of Howard Lasnik, Cambridge: MIT Press.

Martineau, France (2009): «Modeling change: a historical sociolinguistics perspective on French negation», in: Durand, Jacques / Kawaguchi, Yuji / Minegishi, Makoto (édd.): Corpus analysis and variation in linguistics, Amsterdam/Philadelphie: Benjamins. 
Martineau, France (éd.) (2009): Modéliser le changement. Les voies du français $(M C V F)$, Université d'Ottawa.

Martineau, France (2011): «NE-absence in declarative and yes / no interrogative contexts. Some patterns of change», in: Larrivée, Pierre / Ingham, Richard P. (édd.): The evolution of negation. Beyond the Jespersen Cycle, 179-208.

Martineau, France / Mougeon, Raymond (2003): «A Sociolinguistic study of the orignis of NE deletion in European and Quebec French», Language 79, 118-152.

Martineau, France / Nadasdi, Terry James (édd.) (2011): Le français en contact: hommages à Raymond Mougeon, Québec: Presses de l'Université Laval.

Martineau, France / Vinet, Marie Thérèse (2005) : «Microvariation in French negation markers: an historical perspective», in: Batllori, Montse / Hernandez, Maria L. / Roca, Francesc (édd.): Grammaticalization and parametric variation, New York: Oxford University Press, 194-205.

Martins, Ana M. (2000) : «A minimalist approach to clitic climbing», in: Costa, João (éd.): Portuguese syntax: new comparative studies, New York/Oxford: Oxford University Press, 169-190.

Massot, Benjamin (2010): «Le patron diglossique de la variation grammaticale en français », Langue Française 168, 87-106.

Mathieu, Eric (2001): «On the nature of French n-words», UCL Working Papers in Linguistics 13, 319-352.

Meillet, Antoine (1912): «L'évolution des formes grammaticales.», Scientia 12, 384400.

Meisner, Charlotte (2013): «La co-variation stable en 'français parlé' existe-elle? Une analyse de corpus », in: Albizu, Cristina / Döhla, Hans-Jörg et al. (édd.): VARIANTE ET VARIÉTÉ VARIANTE E VARIETÀ - VARIANTE Y VARIEDAD VARIANTE UND VARIETÄT Actes du VIe Dies Romanicus Turicensis, Zurich, 24-25 juin 2011, Pisa: ETS, 51-72.

Meisner, Charlotte (2008): Die Realisierung und Auslassung der Negationspartikel ne im gegenwartssprachlichen Französisch. Eine Analyse sprachinterner und externer Faktoren anhand eines Korpus und einer Sprecherbefragung. Thèse de licence inédite, Freie Universität Berlin.

Meisner, Charlotte (2010): «A corpus analysis of intra- and extralinguistic factors triggering ne-deletion in phonic French», in: Neveu, Frank / Muni Toke, Valelia et al. (édd.) : Congrès Mondial de Linguistique Française - CMLF 2010, Paris : Institut de Linguistique Française, 1943-1962.

Meisner, Charlotte (2013): «Ne oder nicht ne? Akzeptabilitätstests zu verschiedenen Realisierungen der französischen Negation in phonischer Nähe- und Distanzsprache», in: Grutschus, Anke / Fesenmeier, Ludwig / Patzelt, Carolin (édd.): Absences(s) - Phänomene sprachlicher Absenz und Möglichkeiten ihrer Analyse, Francfort s.-1.-M. : Klostermann, 109-130.

Meisner, Charlotte / Pomino, Natascha (2014): «A DM Approach to synchronic variation in French negation», Journal of French Language Studies 24.1, 9-28. 
Mensching, Guido (2008): «Nähesprache versus Distanzsprache: Überlegungen im Rahmen der generativen Grammatik», in: Stark, Elisabeth / Schmidt-Riese, Roland / Stoll, Eva (édd.): Romanische Syntax im Wandel, Tubingue: Narr, 1-29.

Miestamo, Matti (2005): Standard negation: the negation of declarative verbal main clauses in a typological perspective, Berlin/New York: de Gruyter.

Miller, Phillip H. / Monachesi, Paola (2003): «Les pronoms clitiques dans les langues romanes», in: Godard, Danièle (éd.): Les langues romanes: problèmes de la phrase simple, Paris: CNRS Éd., 67-123.

Miller, Phillip H. / Sag, Ivan A. (1997): «French clitic movement without clitics or movement», in: Natural Language and Linguistic Theory 15, 573-639.

Moeschler, Jacques (1982): Dire et contredire. Pragmatique de la négation et acte de réfutation dans la conversation, Berne: Lang.

Moreau, Marie-Louise (1986): «Les séquences préformées: entre les combinaisons libres et les idiomatismes. Le cas de la négation avec ou sans ne», Le Français Moderne 54, 137-160.

Morin, Yves-Charles (1979): «La morphophonologie des pronoms clitiques en français populaire», Cahiers de linquistique 9, 1-36.

Morin, Yves-Charles (1981): «Some myths about pronominal clitics in French», Linguistic analysis 8, 95-109.

Muller, Claude (1991): La négation en français. Syntaxe, sémantique et éléments de comparaison avec les autres langues romanes, Genève: Droz.

Müller, Bodo / Elsass, Annie (1985): Le français d'aujourd'hui, Paris : Klincksieck.

Mussafia, Adolf (1886): «Una particolarità sintattica della lingua italiana dei primi secoli», in: Miscellanea di filologia e linguistica in memoria di N. Caix et U.A. Canello, Florence: Le Monnier, 255-261.

Nash, Rose (1973): Turkish intonation. An instrumental study, La Hague: Mouton.

Neveu, Frank / Muni Toke, Valelia et al. (édd.) (2010): Congrès Mondial de Linguistique Française - CMLF 2010, Paris: Institut de Linguistique Française.

Núñez-Cedeño, Rafael / López, Luis / Cameron, Richard (édd.) (2002): A Romance perspective on language knowledge and use, Amsterdam/Philadelphie: Benjamins.

Overdiep, Gerrit S. (1937): Stilistische Grammatica van het Moderne Nederlandsch, Zwolle: Tjeenk Willink.

Payne, John R. (1985): «Negation», Language Typology and Syntactic Description $1,197-242$.

Penka, Doris (2011): Negative indefinites, Oxford: Oxford University Press.

Pérez, Ana T. / Roberge, Yves (édd.) (2003): Romance linguistics: theory and acquisition, Amsterdam/Philadelphie: Benjamins.

Perlmutter, David M. (1971): Deep and surface structure constraints in Syntax, New York et al. : Holt, Rinehart and Winston.

Peters, Hugues (1999): «An alternative proposal for French negation», Linguistica Atlantica 21, 107-136. 
Pierrehumbert, Janet B. (1980): The phonology and phonetics of English intonation. Thèse de doctorat, MIT Boston: (publiée en 1988 par IULC).

Pohl, Jacques (1968): «Ne dans le français contemporain: les modalités de son abandon», in: Actes du XIe Congrès International de Linguistique et de Philologie Romanes 3.

Pohl, Jacques (1972): L'homme et le signifiant, Paris: Nathan.

Pollock, Jean-Yves (1989): «Verb movement, universal grammar, and the structure of IP», Linguistic Inquiry 20, 365-424.

Pomino, Natascha (2009): Grundlegende Aspekte französischer Objektklitika im Rahmen der Distributed Morphology. [communication du 6 janvier 2009, Freie Universität Berlin, manuscrit inédit.

Pooley, Timothy (1996): Chtimi. The urban vernaculars of northern France, Clevedon: Multilingual Matters.

Poplack, Shana / St-Amand, Anne (2009): «Les Récits du français québécois d'autrefois: reflet du parler vernaculaire du 19e siècle» Canadian Journal of Linguistics / Revue canadienne de linguistique 54, 511-546.

Posner, Rebecca (1985): «Post-verbal negation in non-standard French: a historical and comparative view», Romance Philology 39, 170-197.

Posner, Rebecca (2007): Linguistic change in french, Oxford: Clarendon.

Pusch, Claus D. (2002): "A survey of spoken language corpora in Romance», in: Pusch, Claus D. / Raible, Wolfgang (édd.): Romanistische Korpuslinguistik Korpora und gesprochene Sprache / Romance corpus linguistics - corpora and spoken language, Tubingue: Narr, 245-264.

Pusch, Claus D. (2007): «Les corpus de linguistique romane en pays germanophones », Revue française de linguistique appliquée 12, 111-124.

Pusch, Claus D. / Kabatek, Johannes / Raible, Wolfgang (édd.) (2005): Korpora und diachrone Sprachwissenschaft II. Corpora and diachronic linguistics II, Tubingue: Narr.

Pusch, Claus D. / Kabatek, Johannes / Raible, Wolfgang (2005) : «Romance Corpus linguistics and language change. An introduction to the present volume», in: Pusch, Claus D. / Kabatek, Johannes / Raible, Wolfgang (édd.) : Korpora und diachrone Sprachwissenschaft II. Corpora and diachronic linguistics II, Tubingue: Narr, 1-10.

Pusch, Claus D. / Raible, Wolfgang (édd.) (2002): Romanistische KorpuslinguistikKorpora und gesprochene Sprache / Romance corpus linguistics-corpora and spoken language, Tubingue: Narr.

Pusch, Claus D. / Raible Wolfgang (2002): «Romance corpus linguistics and spoken language. An introduction to the present volume», in: Pusch, Claus D. / Raible, Wolfgang (édd.): Romanistische Korpuslinguistik - Korpora und gesprochene Sprache / Romance corpus linguistics-corpora and spoken language, Tubingue: Narr, 1-6.

Pustka, Elissa (2011): Einführung in die Phonetik und Phonologie des Französischen, Berlin: Erich Schmidt. 
Pustka, Elissa / Eychenne, Julien (2007): «The initial position in southern French: elision, suppletion, emergence», in: Crouzet, Olivier / Angoujard, Jean-Pierre (édd.): Actes des / Proceedings of JEL 2007, Nantes: Université de Nantes, 199204.

Ramat, Paolo / Roma, Elisa (édd.) (1998): Sintassi Storica. Atti del congresso internazionale della società di linguistica italiana, Pavia, 26-28 settembre 1996, Rome: Bulzoni.

Remberger, Eva-Maria (2006): Hilfsverben. Eine minimalistische Analyse am Beispiel des Italienischen und Sardischen, Tubingue: Niemeyer.

Rey, Alain (2007): L'Amour du français. Contre les puristes et autres censeurs de la langue, Paris: Denoël.

Riegel, Martin / Pellat, Jean-Christophe / Rioul, René (52008): Grammaire méthodique du français, Paris: Presses Universitaires de France (Quadrige. Manuel).

Rivero, María Luisa (1997): «On two locations for complement clitic pronouns : SerboCroatian, Bulgarian and Old Spanish», in: van Kemenade, Ans / Vincent, Nigel (édd.): Parameters of morphosyntactic change, Cambridge: Cambridge University Press, 170-206.

Rizzi, Luigi (1986): «Null objects in Italian and the theory of pro», Linguistic Inquiry $17,501-557$.

Rizzi, Luigi (1997): «The fine structure of the left periphery», in: Haegeman, Liliane (éd.): Elements of grammar: a handbook of generative syntax, Dordrecht: Kluwer, 281-337.

Rizzi, Luigi (éd.) (2004): The structure of CP and IP: the cartography of syntactic structures, (vol. 2), Oxford: Oxford University Press.

Roberge, Yves (1990): The syntactic recoverability of null arguments, Montreal: McGill-Queen's University Press.

Roberts, Ian (2007): Diachronic syntax, Oxford/New York: Oxford University Press. Roberts, Ian / Roussou, Anna (1999): «A formal approach to "grammaticalization"», Linguistics 37, 1011-1041.

Rowlett, Paul (2014): «French imperatives, negative ne, and non-subject clitics», Journal of French Language Studies 24.1, 29-47.

Rowlett, Paul (1998): Sentential negation in French, New York/Oxford: Oxford University Press.

Rowlett, Paul (2007): The Syntax of French, Cambridge: Cambridge University Press.

Rowlett, Paul (2013): «Do French speakers really have two grammars?», Journal of French language Studies 23, 1-16.

Sankoff, Gillian / Vincent, David (édd.) (1980): The social life of language, Philadelphia: University of Pennsylvania Press.

Sankoff, Gillian / Vincent, David (1980): «The productive use of NE in spoken Montreal French», in: Sankoff, Gillian / Vincent, David (édd.): The social life of language, Philadelphia: University of Pennsylvania Press, 295-310. 
Schuetze-Coburn, Stephan (1987): Topic management and the lexicon: a discourse profile of three-argument verbs in German, Los Angeles: UCLA.

Séguin, Jean-Pierre (1972): La langue française au 18e siècle, Paris: Bordas.

Shlonsky, Ur (2004): «Enclisis and proclisis», in: Rizzi, Luigi (éd.): The structure of $C P$ and IP: the cartography of syntactic structures, Oxford: Oxford University Press., 329-353.

Simon, Anne Catherine / Auchlin, Antoine et al. (2010): «Les phonostyles. Une description prosodique des styles de parole en français», in: Abecassis, Michaël / Ledegen, Gudrun (édd.): Les voix des Français. En parlant, en écrivant, vol. 2, Berne: Lang, 71-88.

Smith, Wendy (1996): «Spoken narrative and preferred clause structure: evidence from modern Hebrew discourse», in: Studies in Language 20, 163-189.

Söll, Ludwig (21980): Gesprochenes und geschriebenes Französisch, Berlin: Schmidt.

Stark, Elisabeth (2012): "Negation marking in French text messages», Linguisticae Investigationes 35 (Special issue on SMS communication: a linguistic approach), 341-366.

Stark, Elisabeth (1997): Voranstellungsstrukturen und 〈topic〉-Markierung im Französischen. Mit einem Ausblick aufs Italienische, Tubingue: Narr.

Stark, Elisabeth (2006): Indefinitheit und Textkohärenz. Entstehung und semantische Strukturierung indefiniter Nominaldetermination im Altitalienischen, Tubingue: Niemeyer.

Stark, Elisabeth / Meisner, Charlotte / Völker, Harald (édd.) (2014): Negation and clitics in French: interaction and variation (= Special issue, Journal of French Language Studies 24.1).

Stark, Elisabeth / Schmidt-Riese, Roland / Stoll, Eva (édd.) (2008) : Romanische Syntax im Wandel, Tubingue: Narr.

Sturm, Joachim (1981): Morpho-syntaktische Untersuchungen zur phrase négative im gesprochenen Französisch. Die Negation mit und ohne NE, Francfort s.-1.-M.: Lang.

Swart, Henriette de (2010): Expression and Interpretation of Negation. An OT Typo$\log y$, Dordrecht: Springer.

Tobler, A. (1912 [1875]) : «Review of J. Le Coultre's De l'ordre des mots dans Chrétien de Troyes», in: Vermischte Beiträge zur französischen Grammatik, Hirzel: Leipzig, 395-414.

Tomlin, Russel S. (éd.) (1987): Coherence and Grounding in Discourse Amsterdam/ Philadelphie: Benjamins.

Trudgill, Peter (2003): A glossary of sociolinguistics, Edinburgh: Edinburgh University Press.

Vaissière, Jacqueline (1997): «Langues, prosodies et syntaxe», Revue du Traitement Automatique des Langues 38, 53-82.

van Compernolle, Rémi A. (2008a): «Language variation in online personal ads from Quebec: The case of ne»,Language@Internet 5, 1-20. 
van Compernolle, Rémi A. (2008b): «Morphosyntactic and phonological constraints on negative particle variation in French-language chat discourse», Language Variation and Change 20, 317-339.

van Compernolle, Rémi A. (2009): «Emphatic NE in informal spoken French and implications for foreign language pedagogy», International Journal of Applied Linguistics 19, 47-65.

van Compernolle, Rémi A. / Williams, Lawrence (2007): «De l'oral à l'électronique : la variation orthographique comme ressource sociostylistique et pragmatique dans le français électronique», Glottopol 10, 56-69.

van der Auwera, Johan (2009): «The Jespersen Cycles», in: van Gelderen, Elly (éd.) : Cyclical Change: Amsterdam/Philadelphie: Benjamins, 35-71.

van der Auwera, Johan (2010): «The diachrony of negation», in: Horn, Laurence R. (éd.): The expression of negation, Berlin: Mouton de Gruyter, 73-110.

van Gelderen, Elly (éd.) (2009): Cyclical change, Amsterdam/Philadelphie: Benjamins.

van Gelderen, Elly (2009): «Cyclical change. An introduction», in: van Gelderen, Elly (éd.): Cyclical Change, Amsterdam/Philadelphie: Benjamins, 1-12.

van Kemenade, Ans / Vincent, Nigel (édd.) (1997): Parameters of morphosyntactic change, Cambridge: Cambridge University Press.

van Riemsdijk, Henk (éd.) (1999): Clitics in the languages of Europe, Berlin/New York: Mouton de Gruyter.

van Riemsdijk, Henk / Rizzi, Luigi (édd.) (1991): Clitics and their hosts. ESF-Eurotype report. Theme group 8: clitics, Tilburg: Tilburg University.

Völker, Harald (2003): Skripta und Variation. Untersuchungen zur Negation und zur Substantivflexion in altfranzösischen Urkunden der Grafschaft Luxemburg (1237-1281), Tubingue: Niemeyer.

Wagner, Robert Léon / Pichon, Jaqueline (1991): Grammaire du français classique et moderne, Paris: Hachette.

Wanner, Dieter (1987): The development of Romance clitic pronouns. From Latin to old romance, Berlin/New York: Mouton de Gruyter.

Welby, Pauline S. (2003): The slaying of Lady Mondegreen, being a study of French tonal association and alignment and their role in speech segmentation. Thèse de doctorat, Ohio State University: publiée en ligne: $<$ https://etd.ohiolink.edu/ ap:10:0::NO:10:P10_ACCESSION_NUM:osu1074614793>.

Werle, Adam (2002): «A typology of negative indefinites», CLS Parasession on $\mathrm{Ne}$ gation and Polarity 38, 1-26.

Wilmet, Marc (21998): Grammaire critique du français, Paris/Bruxelles: De Boeck \& Larcier Dép. Duculot.

Wilmet, Marc (2007): Grammaire renovée du français, Bruxelles: De Boeck \& Larcier.

Woehrling, Cécile / Boula Mareüil, Philippe de / Adda-Decker, Martine / Lamel, Lori (2008): «A corpus-based prosodic study of Alsatian, Belgian and Swiss 
French», Interspeech 2008 - 9th Annual Conference of the International Speech Communication Association, Brisbane, Australia, September 22-26, 2008 / ISCA Archive, 780-783.

Zeijlstra, Hedde (2004): Sentential negation and negative concord. Thèse de doctorat, University of Amsterdam, publiée en ligne: $<$ http://www.lotpublications.nl/ publish/articles/001108/bookpart.pdf>.

Zribi-Hertz, Anne (1994): "The syntax of nominative clitics in standard and advanced French», in: Cinque, Guglielmo / Koster, Jan et al. (édd.): Paths towards universal grammar. Studies in honor of Richard S. Kayne, Washington D.C.: Georgetown University Press, 453-472.

Zribi-Hertz, Anne (2011): «Pour un modèle diglossique de description du français: quelques implications théoriques, didactiques et méthodologiques», Journal of French language Studies 21, 1-26.

Zwicky, Arnold M. (1977): «On clitics», in: Indiana University linguistcs club, 1-40. Zwicky, Arnold M. / Pullum, Geoffrey K. (1983): «Clitization vs. Inflection. English $\mathrm{N}^{\prime} \mathrm{T} »$, Language 59, 502-513. 


\section{Annexe}

L'annexe peut être trouvée en ligne sous: $<$ http://server.linguistik.uzh. ch/cmeisner2013-tzero>. 
Charlotte Meisner - 978-3-0343-2332-1

Downloaded from PubFactory at 01/11/2019 02:38:59AM

via free access 
Favoriser la confrontation interdisciplinaire et internationale de toutes les formes de recherches consacrées à la communication humaine, en publiant sans délai des travaux scientifiques d'actualité: tel est le rôle de la collection Sciences pour la communication. Elle se propose de réunir des études portant sur tous les langages, naturels ou artificiels, et relevant de toutes les disciplines sémiologiques: linguistique, psychologie ou sociologie du langage, sémiotiques diverses, logique, traitement automatique, systèmes formels, etc. Ces textes s'adressent à tous ceux qui voudront, à quelque titre que ce soit et où que ce soit, se tenir au courant des développements les plus récents des sciences du langage.

Ouvrages parus

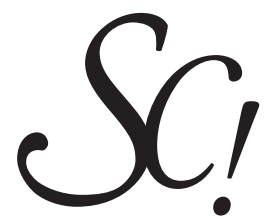


1. Alain Berrendonner - L'éternel grammairien Etude du discours normatif, 1982 (épuisé)

2. Jacques Moeschler-Dire et contredire Pragmatique de la négation et acte de réfutation dans la conversation, 1982 (épuisé)

3. C. Bertaux, J.-P. Desclés, D. Dubarle, Y. Gentilhomme, J.-B. Grize, I. Mel'Cuk, P. Scheurer et R. Thom - Linguistique et mathématiques Peut-on construire un discours cohérent en linguistique? · Table ronde organisée par l'ATALA, le Séminaire de philosophie et mathématiques de l'Ecole Normale Supérieure de Paris et le Centre de recherches sémiologiques de Neuchâtel (Neuchâtel, 29-31 mai 1980), 1982

4. Marie-Jeanne Borel, Jean-Blaise Grize et Denis Miéville - Essai de logique naturelle, 1983, 1992

5. P. Bange, A. Bannour, A. Berrendonner, O. Ducrot, J. Kohler-Chesny, G. Lüdi, Ch. Perelman, B. Py et E. Roulet - Logique, argumentation, conversation · Actes du Colloque de pragmatique (Fribourg, 1981), 1983

6. Alphonse Costadau: Traité des signes (tome I) - Edition établie, présentée et annotée par Odile Le Guern-Forel, 1983

7. Abdelmadjid Ali Bouacha - Le discours universitaire · La rhétorique et ses pouvoirs, 1984

8. Maurice de Montmollin - L'intelligence de la tâche · Eléments d'ergonomie cognitive, 1984, 1986 (épuisé)

9. Jean-Blaise Grize (éd.) - Sémiologie du raisonnement - Textes de D. Apothéloz, M.-J. Borel, J.-B. Grize, D. Miéville, C. Péquegnat, 1984

10. Catherine Fuchs (éd.) - Aspects de l'ambiguïté et de la paraphrase dans les langues naturelles Textes de G. Bès, G. Boulakia, N. Catach, F. François, J.-B. Grize, R. Martin, D. Slakta, 1985

11. E. Roulet, A. Auchlin, J. Moeschler, C. Rubattel et M. Schelling - L'articulation du discours en français contemporain, 1985, 1987, 1991 (épuisé)

12. Norbert Dupont - Linguistique du détachement en français, 1985

13. Yves Gentilhomme - Essai d'approche microsystémique · Théorie et pratique · Application dans le domaine des sciences du langage, 1985

14. Thomas Bearth - L'articulation du temps et de l'aspect dans le discours toura, 1986

15. Herman Parret - Prolégomènes à la théorie de l'énonciation · De Husserl à la pragmatique, 1987

16. Marc Bonhomme - Linguistique de la métonymie · Préface de M. Le Guern, 1987 (épuisé)

17. Jacques Rouault - Linguistique automatique $\cdot$ Applications documentaires, 1987

18. Pierre Bange (éd.) - L'analyse des interactions verbales: «La dame de Caluire. Une consultation» · Actes du Colloque tenu à l'Université Lyon II (13-15 décembre 1985), 1987

19. Georges Kleiber - Du côté de la référence verbale · Les phrases habituelles, 1987 
20. Marianne Kilani-Schoch - Introduction à la morphologie naturelle, 1988

21. Claudine Jacquenod - Contribution à une étude du concept de fiction, 1988

22. Jean-Claude Beacco - La rhétorique de l'historien · Une analyse linguistique du discours, 1988

23. Bruno de Foucault - Les structures linguistiques de la genèse des jeux de mots, 1988

24. Inge Egner-Analyse conversationnelle de l'échange réparateur en wobé · Parler WEE de Côte d'Ivoire, 1988

25. Daniel Peraya - La communication scalène · Une analyse sociosémiotique de situations pédagogiques, 1989

26. Christian Rubattel (éd.) - Modèles du discours • Recherches actuelles en Suisse romande - Actes des Rencontres de linguistique française (Crêt-Bérard, 1988), 1989

27. Emilio Gattico - Logica e psicologia · Studi piagettiani e postpiagettiani, 1989

28. Marie-José Reichler-Béguelin (éd.) - Perspectives méthodologiques et épistémologiques dans les sciences du langage · Actes du Colloque de Fribourg (11-12 mars 1988), 1989

29. Pierre Dupont - Eléments logico-sémantiques pour l'analyse de la proposition, 1990

30. Jacques Wittwer - L'analyse relationnelle · Une physique de la phrase écrite · Introduction à la psychosyntagmatique, 1990

31. Michel Chambreuil et Jean-Claude Pariente - Langue naturelle et logique ' La sémantique intentionnelle de Richard Montague, 1990

32. Alain Berrendonner et Herman Parret (éds) - L'interaction communicative, 1990 (épuisé)

33. Jacqueline Bideaud et Olivier Houdé - Cognition et développement · Boîte à outils théoriques · Préface de Jean-Blaise Grize, 1991 (épuisé)

34. Beat Münch - Les constructions référentielles dans les actualités télévisées · Essai de typologie discursive, 1992

35. Jacques Theureau - Le cours d'action Analyse sémio-logique · Essai d'une anthropologie cognitive située, 1992 (épuisé)

36. Léonardo Pinsky $(\dagger)$ - Concevoir pour l'action et la communication · Essais d'ergonomie cognitive $\cdot$ Textes rassemblés par Jacques Theureau et collab., 1992

37. Jean-Paul Bernié - Raisonner pour résumer · Une approche systémique du texte, 1993

38. Antoine Auchlin - Faire, montrer, dire - Pragmatique comparée de l'énonciation en français et en chinois, 1993

39. Zlatka Guentcheva - Thématisation de l'objet en bulgare, 1993

40. Corinne Rossari - Les opérations de reformulation · Analyse du processus et des marques dans une perspective contrastive français - italien, 1993, 1997

41. Sophie Moirand, Abdelmadjid Ali Bouacha, Jean-Claude Beacco et André Collinot (éds) - Parcours linguistiques de discours spécialisés · Colloque en Sorbonne les 2324-25 septembre 1992, 1994, 1995 
42. Josiane Boutet - Construire le sens · Préface de Jean-Blaise Grize, 1994, 1997

43. Michel Goyens - Emergence et évolution du syntagme nominal en français, 1994

44. Daniel Duprey - L'universalité de «bien» - Linguistique et philosophie du langage, 1995

45. Chantal Rittaud-Hutinet - La phonopragmatique, 1995

46. Stéphane Robert (éd.) - Langage et sciences humaines: propos croisés · Actes du colloque «Langues et langages» en hommage à Antoine Culioli (Ecole normale supérieure. Paris, 11 décembre 1992), 1995

47. Gisèle Holtzer - La page et le petit écran: culture et télévision · Le cas d'Apostrophes, 1996

48. Jean Wirtz - Métadiscours et déceptivité · Julien Torma vu par le Collège de 'Pataphysique, 1996

49. Vlad Alexandrescu - Le paradoxe chez Blaise Pascal • Préface de Oswald Ducrot, 1997

50. Michèle Grossen et Bernard Py (éds) - Pratiques sociales et médiations symboliques, 1997

51. Daniel Luzzati, Jean-Claude Beacco, Reza Mir-Samii, Michel Murat et Martial Vivet (éds) - Le Dialogique · Colloque international sur les formes philosophiques, linguistiques, littéraires, et cognitives du dialogue (Université du Maine, 15-16 septembre 1994), 1997

52. Denis Miéville et Alain Berrendonner (éds) - Logique, discours et pensée · Mélanges offerts à Jean-Blaise Grize, 1997, 1999

53. Claude Guimier (éd.) - La thématisation dans les langues · Actes du colloque de Caen, 9-11 octobre 1997, 1999, 2000

54. Jean-Philippe Babin - Lexique mental et morphologie lexicale, 1998, 2000

55. Thérèse Jeanneret - La coénonciation en français · Approches discursive, conversationnelle et syntaxique, 1999

56. Pierre Boudon - Le réseau du sens · Une approche monadologique pour la compréhension du discours, 1999 (épuisé)

58. Jacques Moeschler et Marie-José Béguelin (éds) - Référence temporelle et nominale. Actes du $3^{\mathrm{e}}$ cycle romand de Sciences du langage, Cluny (15-20 avril 1996), 2000

59. Henriette Gezundhajt - Adverbes en -ment et opérations énonciatives · Analyse linguistique et discursive, 2000

60. Christa Thomsen - Stratégies d'argumentation et de politesse dans les conversations d'affaires · La séquence de requête, 2000

61. Anne-Claude Berthoud et Lorenza Mondada (éds) - Modèles du discours en confrontation, 2000

62. Eddy Roulet, Anne Grobet, Laurent Filliettaz, avec la collaboration de Marcel Burger - Un modèle et un instrument d'analyse de l'organisation du discours, 2001

63. Annie Kuyumcuyan - Diction et mention Pour une pragmatique du discours narratif, 2002 
64. Patrizia Giuliano - La négation linguistique dans l'acquisition d'une langue étrangère · Un débat conclu? 2004

65. Pierre Boudon - Le réseau du sens II · Extension d'un principe monadologique à l'ensemble du discours, 2002

66. Pascal Singy (éd.) - Le français parlé dans le domaine francoprovençal · Une réalité plurinationale, 2002

67. Violaine de Nuchèze et Jean-Marc Colletta (éds) - Guide terminologique pour l'analyse des discours · Lexique des approches pragmatiques du langage, 2002

68. Hanne Leth Andersen et Henning Nølke - Macro-syntaxe et macro-sémantique · Actes du colloque international d' ̊̊rhus, 17-19 mai 2001, 2002

69. Jean Charconnet - Analogie et logique naturelle - Une étude des traces linguistiques du raisonnement analogique à travers différents discours, 2003

70. Christopher Laenzlinger - Initiation à la Syntaxe formelle du français · Le modèle Principes et Paramètres de la Grammaire Générative Transformationnelle, 2003

71. Hanne Leth Andersen et Christa Thomsen (éds) - Sept approches à un corpus · Analyses du français parlé, 2004

72. Patricia Schulz - Description critique du concept traditionnel de «métaphore», 2004

73. Joël Gapany - Formes et fonctions des relatives en français · Etude syntaxique et sémantique, 2004

74. Anne Catherine Simon - La structuration prosodique du discours en français · Une approche mulitdimensionnelle et expérientielle, 2004

75. Corinne Rossari, Anne Beaulieu-Masson, Corina Cojocariu et Anna Razgouliaeva Autour des connecteurs · Réflexions sur l'énonciation et la portée, 2004

76. Pascal Singy (éd.) - Identités de genre, identités de classe et insécurité linguistique, 2004

77. Liana Pop - La grammaire graduelle, à une virgule près, 2005

78. Injoo Choi-Jonin, Myriam Bras, Anne Dagnac et Magali Rouquier (éds) - Questions de classification en linguistique: méthodes et descriptions · Mélanges offerts au Professeur Christian Molinier, 2005

79. Marc Bonhomme - Le discours métonymique, 2005

80. Jasmina Milićević - La paraphrase · Modélisation de la paraphrase langagière, 2007

81. Gilles Siouffi et Agnès Steuckardt (éds) - Les linguistes et la norme · Aspects normatifs du discours linguistique, 2007

82. Agnès Celle, Stéphane Gresset et Ruth Huart (éds) - Les connecteurs, jalons du discours, 2007

83. Nicolas Pepin - Identités fragmentées · Eléments pour une grammaire de l'identité, 2007

84. Olivier Bertrand, Sophie Prévost, Michel Charolles, Jacques François et Catherine Schnedecker (éds) - Discours, diachronie, stylistique du français • Etudes en hommage à Bernard Combettes, 2008 
85. Sylvie Mellet (dir.) - Concession et dialogisme · Les connecteurs concessifs à l'épreuve des corpus, 2008

86. Benjamin Fagard, Sophie Prévost, Bernard Combettes et Olivier Bertrand (éds) Evolutions en français · Etudes de linguistique diachronique, 2008

87. Denis Apothéloz, Bernard Combettes et Franck Neveu (éds) - Les linguistiques du détachement ' Actes du colloque international de Nancy (7-9 juin 2006), 2009

88. Aris Xanthos - Apprentissage automatique de la morphologie $\cdot$ Le cas des structures racine-schème, 2008

89. Bernard Combettes, Céline Guillot, Evelyne Oppermann-Marsaux, Sophie Prévost et Amalia Rodríguez Somolinos (éds) - Le changement en français • Etudes de linguistique diachronique, 2010

90. Camino Álvarez Castro, Flor $\mathrm{M}^{\mathrm{a}}$ Bango de la Campa et María Luisa Donaire (éds) Liens linguistiques · Etudes sur la combinatoire et la hiérarchie des composants, 2010

91. Marie-José Béguelin, Mathieu Avanzi et Gilles Corminboeuf (éds) - La Parataxe • Entre dépendance et intégration; Tome 1, 2010

92. Marie-José Béguelin, Mathieu Avanzi et Gilles Corminboeuf (éds) - La Parataxe • Structures, marquages et exploitations discursives; Tome 2, 2010

93. Nelly Flaux, Dejan Stosic et Co Vet (éds) - Interpréter les temps verbaux, 2010

94. Christian Plantin - Les bonnes raisons des émotions • Principes et méthode pour l'étude du discours émotionné, 2011

95. Dany Amiot, Walter De Mulder, Estelle Moline et Dejan Stosic (éds) - Ars Grammatica . Hommages à Nelly Flaux, 2011.

96. André Horak (éd.) - La litote · Hommage à Marc Bonhomme, 2011.

97. Franck Neveu, Nicole Le Querler et Peter Blumenthal (éds) - Au commencement était le verbe. Syntaxe, sémantique et cognition · Mélanges en l'honneur du Professeur Jacques François, 2011.

98. Louis de Saussure et Alain Rihs (éds) - Etudes de sémantique et pragmatique françaises, 2012.

99. L. de Saussure, A. Borillo et M. Vuillaume (éds) - Grammaire, lexique, référence. Regards sur le sens · Mélanges offerts à Georges Kleiber pour ses quarante ans de carrière, 2012.

100. Groupe de Fribourg - Grammaire de la période, 2012

101. C. Guillot, B. Combettes, A. Lavrentiev, E. Oppermann-Marsaux et S. Prévost (éd.) Le changement en français · Etudes de linguistique diachronique, 2012.

102. Gudrun Vanderbauwhede - Le déterminant démonstratif en français et en néerlandais • Théorie, description, acquisition, 2012.

103. Genoveva Puskás - Initiation au Programme Minimaliste · Eléments de syntaxe comparative, 2013.

104. Coco Norén, Kerstin Jonasson, Henning Nølke et Maria Svensson (éds) - Modalité, évidentialité et autres friandises langagières · Mélanges offerts à Hans Kronning à l'occasion de ses soixante ans, 2013. 
105. Jean-Claude Anscombre, María Luisa Donaire et Pierre Patrick Haillet (éds) - Opérateurs discursifs du français · Eléments de description sémantique et pragmatique, 2013.

106. Laurent Gosselin, Yann Mathet, Patrice Enjalbert et Gérard Becher (éds) - Aspects de l'itération · L'expression de la répétition en français: analyse linguistique et formalisation, 2013

107. Alain Rihs - Subjonctif, gérondif et participe présent en français · Une pragmatique de la dépendance verbale, 2013.

108. Emmanuelle Labeau and Jacques Bres (éds) - Evolution in Romance Verbal Systems, 2013

109. Alda Mari - Modalités et Temps · Des modèles aux données, 2015.

110. Christiane Soum-Favaro, Annelise Coquillon et Jean-Pierre Chevrot (éds) - La liaison: approches contemporaines, 2014.

111. Marion Fossard et Marie-José Béguelin (éds) - Nouvelles perspectives sur l'anaphore · Points de vue linguistique, psycholinguistique et acquisitionnel, 2014.

112. Thierry Herman et Steve Oswald (éds.) - Rhétorique et cognition / Rhetoric and Cognition, 2014.

113. Giovanni Gobber and Andrea Rocci (éds) - Language, reason and education, 2014 • Studies in honor of Eddo Rigotti, 2014.

114. Elena Siminiciuc - L'ironie dans la presse satirique - Etude sémantico-pragmatique, 2015.

115. Milton N. Campos - Traversée · Essai sur la communication, 2015.

116. Gaétane Dostie \& Pascale Hadermann (éds) - La dia-variation en français actuel · Etudes sur corpus, approches croisées et ouvrages de référence, 2015.

117. Anne Carlier, Michèle Goyens \& Béatrice Lamiroy (éds) - Le français en diachronie • Nouveaux objets et méthodes, 2015.

118. Charlotte Meisner - La variation pluridimensionnelle $\cdot$ Une analyse de la négation en français, 2016.

119. Laurence Rouanne \& Jean-Claude Anscombre - Histoires de dire $\cdot$ Petit glossaire des marqueurs formés sur le verbe dire, 2016. 
Charlotte Meisner - 978-3-0343-2332-1

Downloaded from PubFactory at 01/11/2019 02:38:59AM

via free access 